\title{
Modelos log-Birnbaum-Saunders mistos
}

\author{
Cristian Marcelo Villegas Lobos
}

\author{
TESE APRESENTADA \\ AO \\ INSTITUTO DE MATEMÁTICA E EsTATÍSTICA \\ DA \\ UNIVERSIDADE DE SÃo PAULO \\ PARA \\ OBTENÇÃO DO TÍTULO \\ $\mathrm{DE}$ \\ DOUTOR EM CIÊNCIAS
}

\section{Programa: Estatística \\ Orientador: Prof. Dr. Gilberto Alvarenga Paula}

Durante o desenvolvimento deste trabalho o autor recebeu auxílio financeiro da Capes e CNPq.

São Paulo, Agosto de 2010 


\section{Modelos log-Birnbaum-Saunders mistos}

Esta versão definitiva da tese/dissertação contém as correções e alterações sugeridas pela

Comissão Julgadora durante a defesa realizada por Cristian Marcelo Villegas Lobos em 6/10/2010.

Comissão Julgadora:

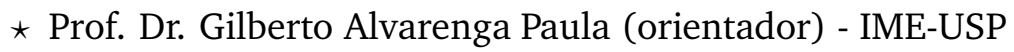

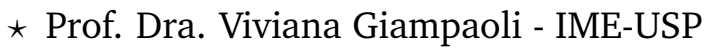

* Prof. Dra. Michelli Karinne Barros da Silva - UFCG

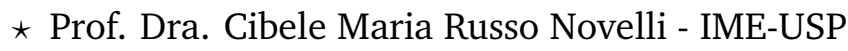

* Prof. Dr. Víctor Eliseo Leiva Sánchez - Universidad de Valparaíso-Chile 


\section{Agradecimentos}

Em primeiro lugar, eu quero agradecer a Deus por ter me dado a força nos momentos de fraqueza.

Eu quero agradecer à minha família, em especial aos meus pais Maritza e Tirso por ter me apoiado ao longo desses anos fora do meu querido pais CHILE e aos meus irmãos Martin e Silvana pela força e pelas palavras constantes de ânimo.

Aos professores Gilberto A. Paula e Víctor Leiva pelas sugestões, conselhos, paciência e por terem confiado em mim ao longo da minha tese.

Às professoras Michelli Barros e Viviana Giampaoli pelas correções feitas no meu trabalho.

A minha namorada e amiga Andrea Estevez (Colombia) pelo carinho, paciência e tolerância quando eu estava finalizando a tese.

Eu quero agradecer também, com muito carinho as minhas duas grandes amigas brasileiras, Cibele Russo Novelli e Lizandra Castilho Fabio; pela amizade e pelas correções realizadas ao português da minha tese.

Eu agradeço muito ao professor Manuel Galea (Chile) pelos seus conselhos e por ter me motivado a continuar estudando e fazer o doutorado aqui no Brasil na qual foi uma experiência muito marcante em minha vida.

Aos meus amigos Lenin Villamizar (Colombia), Alvaro Galdos (Peru), Hugo Tiburtino (Brasil, Recife) pelo carinho e amizade.

Aos professores do IME especialmente Silvia Ferrari, Lúcia Pereira Barroso, Denise Aparecida Botter, Antonio Carlos Pedroso de Lima, Carlos Alberto de Bragança Pereira e Daisy Gomes de Souza Tu.

Aos meus amigos que ficaram em Chile, Víctor Espejo, Jorge Leiva e Marybel Silva pela constante preocupação. 
A comunidade chilena do IME, Marco Riquelme (Talca), Mario Rojas e Pedro Cortes (Antofagasta), Manuel González (Loncoche), Diego Gallardo (Melipilla) e Alejandra Tapia (Valparaíso). Aos chilenos fora do IME Fernanda e Tatiana (Santiago), Patricia e Marcelo (Viña del Mar), Juan Claudio e Millaray (Arica), Alvaro e Mónica (Temuco), Mario e Verónica (Santiago) e Andres (Santiago).

Eu quero agradecer as pessoas que me ajudaram muito na tese, ao meu amigo chileno German Ibacache pelas conversas que tivemos nestes 4 anos. O Gustavo Henrique de Araujo Pereira (Brasil), Dalton (Brasil, Corinthiano) e Jalmar Carrasco (Peru) pela imensa ajuda no SAS. Ao Gerard Alva Morales (Peru) pelas conversas que tivemos dos polinômios de Hermite. Ao professor Edwin Ortega (ESALQ) pela ajuda nas simulações dos resíduos, a professora Gisela Tunes (IME-USP) pela conversa em relação aos modelos de Cox, ao professor Felipe Osorio (UV, Chile) pela ajuda na teoria dos modelos mistos e aproximações de Laplace e a professora Viviana Giampaoli (IME-USP) pelas conversas em relação ao teste de componente de variânzas.

Eu também gostaria de agradecer todas as pessoas que indiretamente contribuirão na elaboração desta tese.

Finalmente, agradeço à Capes e CNPq pelo apoio financeiro para a realização deste trabalho. 


\section{Resumo}

O objetivo principal deste trabalho é introduzir os modelos log-Birnbaum-Saunders mistos (log-BS mistos) e estender os resultados para os modelos log-Birnbaum-Saunders t-Student mistos (logBS-t mistos). Os modelos log-BS são bastante conhecidos desde o trabalho de Rieck e Nedelman (1991) e particularmente receberam uma grande atenção nos últimos 10 anos com vários trabalhos publicados em periódicos internacionais. Contudo, o enfoque desses trabalhos tem sido em modelos log-BS ou log-BS generalizados com efeitos fixos, não havendo muita atenção para modelos com efeitos aleatórios. Inicialmente, apresentamos no trabalho uma revisão das distribuições Birnbaum-Saunders e Birnbaum-Saunders generalizada (BSG) e em seguida discutimos os modelos log-BS e log-BS-t com efeitos fixos, para os quais revisamos alguns resultados de estimação e diagnóstico. Os modelos log-BS mistos são então apresentados precedidos de uma revisão dos métodos de quadratura de Gauss-Hermite (QGH). Embora a estimação dos parâmetros nos modelos log-BS mistos seja efetuada através do procedimento Proc NLMIXED do SAS (Littell et al., 1996), aplicamos o método de quadratura não adaptativa a fim de obtermos aproximações para o logaritmo da função de verossimilhança do modelo log-BS de intercepto aleatório. Com essas aproximações derivamos as funções escore e a matriz de informação observada, além das curvaturas normais de influência local (Cook, 1986) para alguns esquemas de perturbação usuais. Os mesmos procedimentos são aplicados para os modelos log-BS-t de intercepto aleatório. Discussões sobre a predição dos efeitos aleatórios, teste para o componente de variância dos modelos com intercepto aleatório e análises de resíduos são também apresentados. Finalmente, comparamos os ajustes de modelos log-BS e log-BS mistos a um conjunto de dados reais. Métodos de diagnóstico são utilizados na comparação dos modelos ajustados. 



\section{Abstract}

The aim of this work is to introduce the log-Birnbaum-Saunders mixed models (log-BS mixed models) and to extend the results to log-Birnbaum-Saunders Student-t mixed models (log-BS-t mixed models). The log-BS models are well-known since the work by Rieck e Nedelman (1991) and particularly have received great attention in the last 10 years with various published papers in international journals. However, the emphasis given in such works has been in fixed-effects models with few attention given to random-effects models. Firstly, we present in this work a review on Birnbaum-Saunders and generalized Birnbaum-Saunders distributions and so we discuss $\log$-BS and log-BS-t fixed-effects models for which some results on estimation and diagnostic are presented. Then, we introduce the log-BS mixed models preceded by a review on Gauss-Hermite quadrature. Although the parameter estimation of the marginal log-BS and log-BS-t mixed models are performed in the procedure NLMIXED of SAS (Littell et al., 1996), we apply the quadrature methods in order to obtain approximations for the likelihood function of the log-BS and log-BS-t random intercept models. These approximations are used to derive the respective score functions, observed information matrices as well as the normal curvature of local influence (Cook, 1986) under some usual perturbation schemes. Discussions on the prediction of the random effects, variance component tests and residual analysis are also given. Finally, we compare the fits of logBS and log-BS-t mixed models to a real data set. Diagnostic methods are used in the comparisons. 



\section{Sumário}

$\begin{array}{lc}\text { Agradecimentos } & \text { i }\end{array}$

Resumo $\quad$ iii

Abstract $\quad$ V

Lista de Figuras

Lista de Tabelas $\quad$ xiii

1 Motivação 1

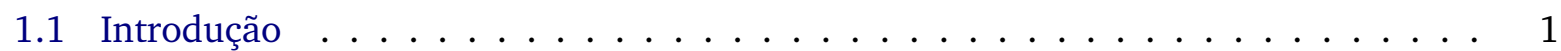

1.2 Conceitos básicos na análise de sobrevivência . . . . . . . . . . . . . 2

1.2.1 Relações entre funções de sobrevivência . . . . . . . . . . . . . . . . . . . . . . . . . . . . .

1.2 .2 Tipos de censura . . . . . . . . . . . . . . . . . 3

1.3 Revisão da literatura . . . . . . . . . . . . . . . . . . . . 4

1.4 Derivação da distribuição Birnbaum-Saunders . . . . . . . . . . . . . . . 5

1.5 Distribuição simétrica univariada . . . . . . . . . . . . . . . 7

1.6 Distribuição seno hiperbólico simétrica . . . . . . . . . . . . . . . . . . . . . . . . . . . .

1.7 Distribuição seno hiperbólico normal . . . . . . . . . . . . . . . . . 9

1.7.1 Propriedades e análise gráfica . . . . . . . . . . . . . . 10

1.7 .2 Função geradora de momentos . . . . . . . . . . . . . . . . 10

1.8 Distribuição seno hiperbólico t . . . . . . . . . . . . . . . . . . . . 12

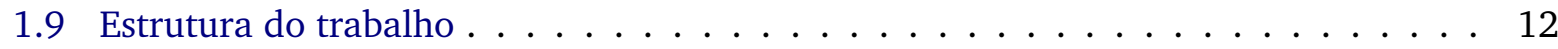

2 Distribuições Birnbaum-Saunders e Birnbaum-Saunders generalizada 15

2.1 Distribuição Birnbaum-Saunders . . . . . . . . . . . . . . . . . 16

2.1.1 Propriedades e análise gráfica . . . . . . . . . . . . . 16

2.2 Distribuição Birnbaum-Saunders generalizada . . . . . . . . . . . . . . . . 18

2.2.1 Propriedades e análise gráfica . . . . . . . . . . . . . . . . . . . . . . . . . . . . . . . . . . . . . . . . . .

2.2 Momentos . . . . . . . . . . . . . . . . . 20

2.3 Distribuição log-Birnbaum-Saunders generalizada . . . . . . . . . . . . . . . 22 
2.3.1 Distribuição log-Birnbaum-Saunders . . . . . . . . . . . . . . . . 22

2.3.2 Distribuição log-Birnbaum-Saunders-t . . . . . . . . . . . . 22

2.4 Conclusões do capítulo . . . . . . . . . . . . . . . . . . . . 23

3 Modelos log-Birnbaum-Saunders e log-Birnbaum-Saunders-t de Efeitos Fixos 25

3.1 Modelos log-Birnbaum-Saunders . . . . . . . . . . . . . . . . 25

3.1 .1 Função escore . . . . . . . . . . . . . . . . . . . . . . . . . . . . . . . . 26

3.1 .2 Matriz hessiana . . . . . . . . . . . . . . . 27

3.1 .3 Resultados assintóticos . . . . . . . . . . . . . . . 27

3.2 Modelos log-Birnbaum-Saunders-t . . . . . . . . . . . . . 28

3.2 .1 Função escore . . . . . . . . . . . . . . . . . . . 28

3.2 .2 Matriz hessiana . . . . . . . . . . . . . . . . . . . . . 29

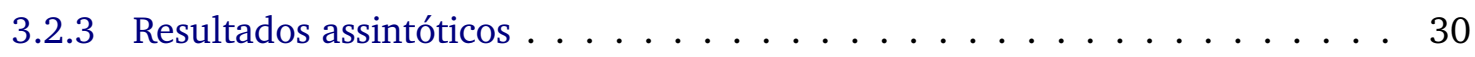

3.3 Análise de diagnóstico . . . . . . . . . . . . . . . . . . 30

3.3 .1 Influência local . . . . . . . . . . . . . 30

3.3 .2 Influência local total . . . . . . . . . . . . . . . 32

3.3 .3 Influência local conformal . . . . . . . . . . . . . . . . . 32

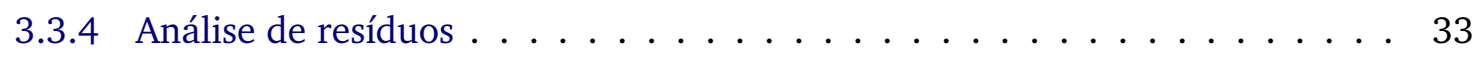

3.4 Conclusões do capítulo . . . . . . . . . . . . . . . . . . . . 34

4 Modelos log-Birnbaum-Saunders mistos $\quad 35$

4.1 Quadratura de Gauss-Hermite . . . . . . . . . . . . . . . . 36

4.1 .1 Versão unidimensional . . . . . . . . . . . . . . . . . 36

4.1 .2 Fórmulas de Gauss-Hermite . . . . . . . . . . . . . . . 37

4.1 .3 Erro nas fórmulas de Gauss-Hermite . . . . . . . . . . . . . . 38

4.1.4 Quantos pontos de quadratura são necessários? . . . . . . . . . . . . . . . . 39

4.1 .5 Versão multidimensional . . . . . . . . . . . . . . . . . . . . 41

4.2 Modelos log-Birnbaum-Saunders mistos . . . . . . . . . . . . . . . . . 42

4.3 Modelos log-Birnbaum-Saunders de intercepto aleatório . . . . . . . . . . . . 43

4.3 .1 Consequências do modelo log-BS . . . . . . . . . . . . . . 44

4.3 .2 Modelo marginal . . . . . . . . . . . . . . . . . . 46

4.3 .3 Função escore . . . . . . . . . . . . . . . . . . . . 47

4.3 .4 Matriz hessiana . . . . . . . . . . . . . . . . . . 49

4.3 .5 Estimação dos parâmetros . . . . . . . . . . . . . . . . . . 52

4.3 .6 Predição do efeito aleatório . . . . . . . . . . . . . . . . . . . . . . . . . . 53

4.3 .7 Teste do componente de variância . . . . . . . . . . . . . . . . 53

4.3 .8 Influência local dos grupos . . . . . . . . . . . . . 54

4.3 .9 Resíduos . . . . . . . . . . . . . . . . . . 63

4.3 .10 Simulações . . . . . . . . . . . . . . . . . . . 64

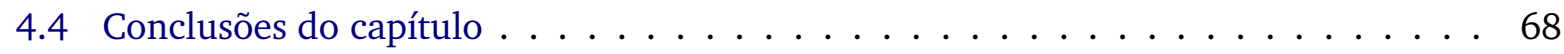


5 Modelos log-Birnbaum-Saunders-t mistos $\quad 69$

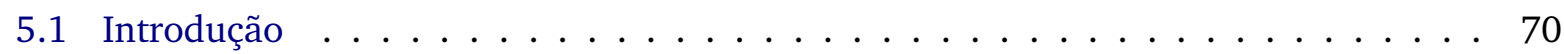

5.2 Modelos log-Birnbaum-Saunders-t mistos . . . . . . . . . . . . . 70

5.3 Modelos log-Birnbaum-Saunders-t de intercepto aleatório . . . . . . . . . . 71

5.3 .1 Modelo marginal . . . . . . . . . . . . . . . 71

5.3 .2 Função escore . . . . . . . . . . . . . . . . . . 71

5.3 .3 Robustez das estimativas . . . . . . . . . . . . . . . 72

5.3 .4 Matriz hessiana . . . . . . . . . . . . . . . 73

5.3 .5 Influência local dos grupos . . . . . . . . . . . . . 75

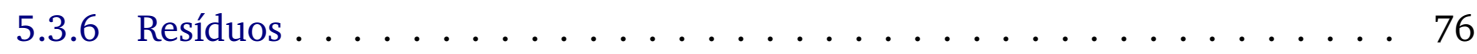

5.4 Conclusões do capítulo . . . . . . . . . . . . . . . . . 77

6 Aplicação $\quad 79$

6.1 Descrição do problema . . . . . . . . . . . . . . . . . . . . . 79

6.2 Modelo $\log$-Birnbaum-Saunders de efeitos fixos . . . . . . . . . . . . . . . . . . . . . . . . .

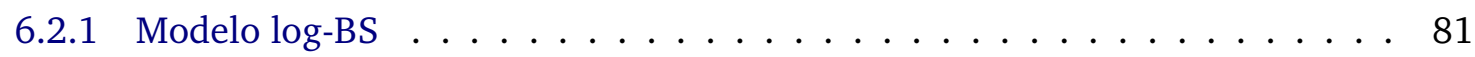

6.2 .2 Modelo log-BS-t . . . . . . . . . . . . . . . 82

6.3 Modelo log-Birnbaum-Saunders de intercepto aleatório . . . . . . . . . . . 83

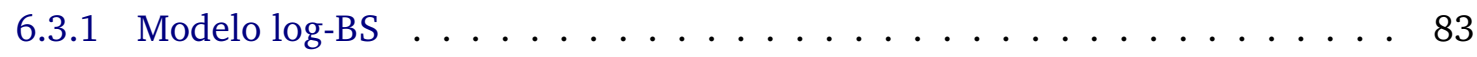

6.3 .2 Modelo log-BS-t . . . . . . . . . . . . . . 85

6.4 Conclusões da aplicação . . . . . . . . . . . . . . . 86

7 Considerações Finais $\quad 89$

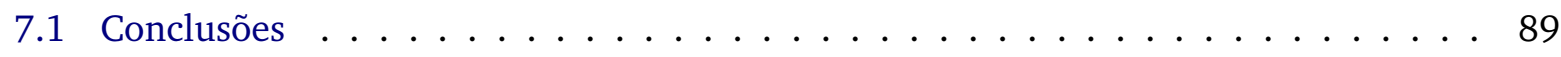

7.2 Perspectivas para trabalhos futuros . . . . . . . . . . . . 89

A Código R baseado no pacote bs $\quad 91$

A.1 Aspectos computacionais . . . . . . . . . . . . . . . . . . 91

B Derivadas usadas nos Capítulos 4 e 5

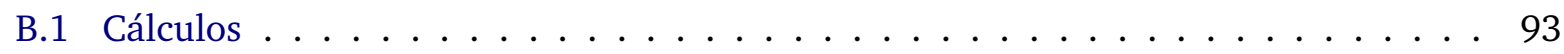

C Relações usadas na Matriz Hessiana $\quad 95$

C.1 Relações entre cosh e senh . . . . . . . . . . . . . . . . . . . . 95

D Matrices $\Delta$ no Modelo log-BS-t de intercepto aleatório 97

D.1 Perturbação aditiva da resposta . . . . . . . . . . . . . . . . . 97

D.2 Perturbação aditiva de uma variável explicativa . . . . . . . . . . . . . . . 99

E Código SAS para os Modelos log-BS e log-BS-t de Intercepto Aleatório 103

E.1 Modelo log-Birnbaum-Saunders de intercepto aleatório . . . . . . . . . . . . . . . 103

E.2 Modelo log-Birnbaum-Saunders-t de intercepto aleatório . . . . . . . . . . . . 103 


\section{Lista de Figuras}

1.1 Ilustração de alguns tipos de censura . . . . . . . . . . . . . . . . . 4

1.2 Gráfico da função densidade de probabilidade seno hiperbólico normal . . . . . . . 11

2.1 Gráfico da fdp e função de risco da distribuição BS . . . . . . . . . . . . . . . 17

2.2 Gráfico da distribuição BSG Cauchy, Laplace, normal e t-Student . . . . . . . . . . . 19

2.3 Gráfico da distribuição BSG exponencial potência, Kotz e normal . . . . . . . . . 20

2.4 Gráfico das distribuições log-BS-t, log-BS, normal e $t_{\nu} \ldots \ldots \ldots$

3.1 Gráfico de $w\left(\xi^{2}\right)$ versus $\xi^{2}$ para diferentes valores de $\nu \ldots \ldots$. . . . . . . . 29

4.1 Resíduo tipo martingale condicional com $10 \%$ de censura . . . . . . . . . . 65

4.2 Resíduo tipo martingale condicional com 30\% de censura . . . . . . . . . . . 66

4.3 Resíduo tipo martingale condicional com $50 \%$ de censura . . . . . . . . . . 67

5.1 Gráfico do resíduo $r_{\mathrm{MT}_{i j}}$ versus o resíduo senh para observações não censuradas 77

6.1 Gráfico de dispersão dos tempos de falha para as fibras (Kevlar 49) . . . . . . . 80

6.2 Boxplots dos tempos de falha considerando os dados de fibras . . . . . . . . . . 80

6.3 Gráfico $r_{\mathrm{MT}_{i j}}$ no modelo log-Birnbaum-Saunders . . . . . . . . . . . . . 82

6.4 Gráfico $l_{\max }$ no modelo log-Birnbaum-Saunders . . . . . . . . . . . . . . 82

6.5 Gráfico $r_{\mathrm{MT}_{i j}}$ no modelo log-Birnbaum-Saunders-t . . . . . . . . . . . . . . . 84

6.6 Gráfico $l_{\max }$ no modelo $\log$-Birnbaum-Saunders-t $\ldots \ldots \ldots$. . . . . . . 84

6.7 Gráfico $r_{\mathrm{MT}_{i j}}$ no modelo log-Birnbaum-Saunders de intercepto aleatório . . . . . . 85

6.8 Gráfico $r_{\mathrm{MT}_{i j}}$ no modelo log-Birnbaum-Saunders-t de intercepto aleatório . . . . . . 86

6.9 Gráficos de perfis ajustados sob o modelo log-BS de intercepto aleatório $\quad$. . . . 87 



\section{Lista de Tabelas}

1.1 Função densidade de probabilidade para algumas distribuições simétricas padrão $\quad 8$

4.1 Valores de $H_{q}(x)$ e $v_{k}$ para $q=2$, 3 e $4 \ldots \ldots \ldots \ldots$

6.1 Ajuste do modelo log-Birnbaum-Saunders $\ldots \ldots \ldots \ldots$. . . . . . . . 81

6.2 Ajuste do modelo log-Birnbaum-Saunders-t . . . . . . . . . . . 83

6.3 Ajuste do modelo log-Birnbaum-Saunders de intercepto aleatório . . . . . . . . 85

6.4 Ajuste do modelo log-Birnbaum-Saunders-t de intercepto aleatório . . . . . . . 86

6.5 Logaritmos dos tempos de falha de fibras (Kevlar 49) . . . . . . . . . . 88 



\section{Capítulo 1}

\section{Motivação}

\subsection{Introdução}

Os modelos lineares com efeitos mistos são bastante utilizados para analisar dados com medidas repetidas, dados agrupados e dados longitudinais, entre outros, sendo de grande aplicabilidade em pesquisas de diversas áreas como agricultura, biologia, economia, geofísica e ciências sociais (veja, por exemplo, Diggle et al., 1994). A crescente popularidade desses modelos é explicada pela flexibilidade que eles oferecem para modelar a correlação entre e intraunidades amostrais, frequentemente presente em dados longitudinais (Laird e Ware, 1982) e dados agrupados (Henderson, 1984), pela capacidade de modelar dados balanceados e desbalanceados e pela existência de programas computacionais eficientes e confiáveis para desenvolvê-los. O modelo linear misto tem como casos particulares o modelo clássico, o modelo de componentes de variância e também os modelos hierárquicos (ou multiníveis).

Uma suposição rotineira, na versão clássica do modelo, é que tanto os erros como os efeitos aleatórios são normalmente distribuídos. Sob essas suposições, inferências para os parâmetros fixos e aleatórios dos modelos podem ser obtidas usando uma ampla variedade de programas, tais como Proc MIXED do SAS (Littell et al., 1996) e a library lme do Splus (Pinheiro e Bates, 2000). Uma das aplicações que o modelo linear misto pode ter é em problemas que envolvem a fadiga de materiais.

A fadiga é um dano estrutural que ocorre quando um material é exposto a flutuações de estresse e tensão. Modelos estatísticos para processos de fadiga de materiais nos possibilitam descrever a variação aleatória dos tempos de falha associados aos materiais expostos à fadiga como um resultado de diferentes padrões e forças cíclicas. Esses materiais podem ser caracterizados por parâmetros de distribuições de vida. Tais caracterizações são importantes para predizer a performance dos materiais sob diferentes condições (veja, por exemplo Galea et al., 2004; Leiva et al., 2007). Entre os modelos probabilísticos que têm sido popularmente propostos para descrever a vida por fadiga encontram-se as distribuições gama, Gaussiana inversa e lognormal, que se ajustam com grande precisão na região central da distribuição de vida.

A seguir revisamos alguns conceitos básicos na análises de sobrevivência que serão usados no trabalho, isto é, a função de sobrevivência, a função de risco e alguns tipos de censura. Detalhes podem ser consultados em Lawless (1982). 


\subsection{Conceitos básicos na análise de sobrevivência}

A seguinte discussão assume que o tempo de falha é uma variável aleatória contínua $T$ com suporte no intervalo $[0, \infty)$ com uma distribuição que depende de um parâmetro $\theta$.

Denotamos a função densidade de probabilidade de $T$ por $f_{T}(t ; \theta)$ e a função de distribuição acumulada de $T$ por $F_{T}(t ; \theta)$ dada por

$$
\begin{aligned}
F_{T}(t ; \theta) & =\int_{0}^{t} f_{T}(x ; \theta) \mathrm{d} x \\
& =P_{\theta}(T \leq t) .
\end{aligned}
$$

Se $\xi_{p}$ é tal que $F_{T}\left(\xi_{p} ; \theta\right)=p$, com $0<p<1$, então $\xi_{p}$ é chamado de $p$-ésimo percentil da distribuição $F_{T}(t ; \theta)$.

A função de distribuição acumulada e a função densidade de probabilidade são usadas na análise estatística para descrever as variáveis aleatórias e estudar as suas propriedades.

Frequentemente ao invés de usar a função de distribuição acumulada, usamos a função de sobrevivência, denotada por $S_{T}(t ; \theta)$ a qual está relacionada à função de distribuição acumulada da seguinte forma:

$$
\begin{aligned}
S_{T}(t ; \theta) & =\int_{t}^{\infty} f_{T}(x ; \theta) \mathrm{d} x \\
& =P_{\theta}(T>t) \\
& =1-F_{T}(t ; \theta),
\end{aligned}
$$

em que $S_{T}(t ; \theta)$ representa a probabilidade que o evento de interesse ocorra após o tempo $t$.

Note que se $\xi_{p}$ é o $p$-ésimo percentil da distribuição $F_{T}(t ; \theta)$, então $S_{T}\left(\xi_{p} ; \theta\right)=1-p$. A função de sobrevivência possui duas propriedades que são

(i) $\quad S_{T}(0 ; \theta)=1$

(ii) $\lim _{t \rightarrow \infty} S_{T}(t ; \theta)=0$.

A função de risco, denotada por $h_{T}(t ; \theta)$, é definida como

$$
\begin{aligned}
h_{T}(t ; \theta) & =\lim _{\Delta t \rightarrow 0} \frac{P_{\theta}(t<T \leq t+\Delta t \mid t<T)}{\Delta t} \\
& =\frac{1}{S_{T}(t ; \theta)} \lim _{\Delta t \rightarrow 0} \frac{F_{T}(t+\Delta t ; \theta)-F_{T}(t ; \theta)}{\Delta t} \\
& =\frac{f_{T}(t ; \theta)}{S_{T}(t ; \theta)} \\
& =\frac{f_{T}(t ; \theta)}{1-F_{T}(t ; \theta)} .
\end{aligned}
$$




\subsubsection{Relações entre funções de sobrevivência}

A seguir apresentamos algumas relações entre as funções $f_{T}(t ; \theta), F_{T}(t ; \theta), h_{T}(t ; \theta)$ e $S_{T}(t ; \theta)$.

$$
\begin{aligned}
f_{T}(t ; \theta) & =\frac{\mathrm{d}}{\mathrm{d} t} F_{T}(t ; \theta), \\
S_{T}(t ; \theta) & =1-F_{T}(t ; \theta) \quad \mathrm{e} \\
h_{T}(t ; \theta) & =-\frac{\mathrm{d}}{\mathrm{d} t} \log \left(1-F_{T}(t ; \theta)\right) .
\end{aligned}
$$

As funções $f_{T}(t ; \theta), F_{T}(t ; \theta), S_{T}(t ; \theta)$ e $h_{T}(t ; \theta)$ são muito importantes para caracterizar o comportamento das diferentes distribuições paramétricas de tempo de vida, como por exemplo, a distribuição de Weibull, exponencial, log-normal, Gaussiana inversa e Birnbaum-Saunders (veja, por exemplo Leiva et al., 2009).

Uma característica frequente na análise de sobrevivência é ter observações incompletas ou parciais. Essas observações, denominadas censuras, podem ocorrer por uma variedade de razões, dentre elas, a perda de acompanhamento do paciente no decorrer do estudo e a não ocorrência do evento. Isto é, a censura se produz quando o tempo até a ocorrência do evento é superior ao tempo registrado até o último acompanhamento. A seguir estudamos alguns tipos de censura.

\subsubsection{Tipos de censura}

Basicamente existem três tipos de censura (veja Figura 1.1). A censura tipo I é aquela em que o estudo é finalizado após um período pré-estabelecido de tempo. A censura tipo II é aquela em que o estudo será finalizado após ter ocorrido o evento de interesse em um número pré-estabelecido de indivíduos. Um terceiro mecanismo de censura é a censura aleatória, em que os tempos de censura são variáveis aleatórias mutuamente independentes e ainda independentes dos tempos de vida. Isso acontece, por exemplo, quando um paciente é retirado no decorrer do estudo sem ter ocorrido a falha, ou também se o paciente morrer por uma causa diferente da estudada. Para mais detalhes e exemplos dos tipos de censura veja, por exemplo, Lawless (1982).

Birnbaum e Saunders (1969a), motivados por problemas de vibração encontrados em aviões comerciais novos e de falhas de materiais, usaram o conhecimento sobre um tipo particular de fadiga para derivar uma nova família de distribuições, a qual modela o tempo de vida de materiais e equipamentos sujeitos a cargas dinâmicas.

A seguir apresentamos a revisão da literatura e derivação da distribuição Birnbaum-Saunders. Posteriormente, mostramos a distribuição simétrica univariada e seno hiperbólico simétrica (SHS). Com base nessa última distribuição são derivadas as distribuições seno hiperbólico normal (SHN) e seno hiperbólico t (SHt), as quais estão relacionadas com as distribuições log-Birnbaum-Saunders (log-BS) e log-Birnbaum-Saunders-t (log-BS-t) que serão indicadas no Capítulo 2. 
(a) Dados completos

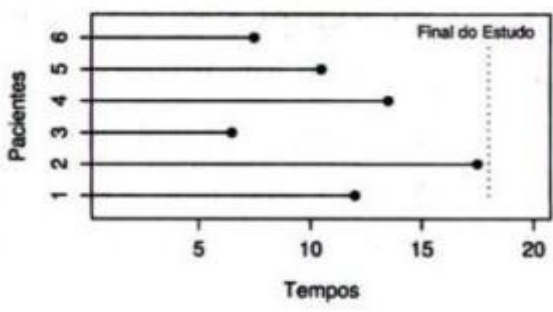

(c) Dados com censura tipo II

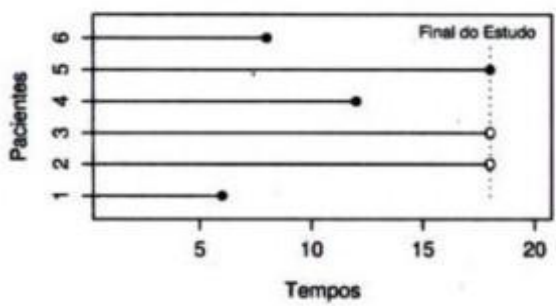

(b) Dados com censura tipo I

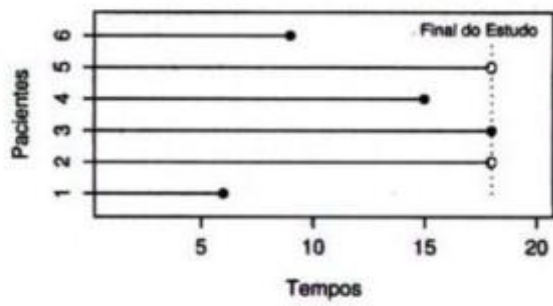

(d) Dados com censura aleatorla

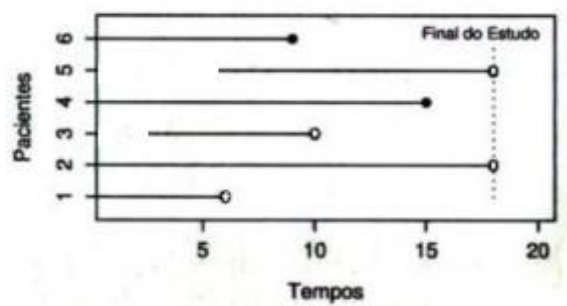

Figura 1.1: Ilustração de alguns tipos de censura em que • representa a falha e $\circ$ a censura (Fonte: Colosimo e Giolo, 2006).

\subsection{Revisão da literatura}

A seguir, citaremos alguns dos principais trabalhos envolvendo a distribuição Birnbaum-Saunders. Birnbaum e Saunders (1969b) obtiveram os estimadores de máxima verossimilhança da distribuição Birnbaum-Saunders. Engelhardt et al. (1981) propuseram intervalos de confiança e testes de hipóteses para os parâmetros da distribuição considerando um deles como parâmetro de perturbação desconhecido. Desmond (1985) aprimorou a justificativa física usada para a construção dessa distribuição, flexibilizando algumas suposições feitas por Birnbaum e Saunders (1969a) além de apresentar uma derivação mais geral baseada em um modelo biológico. Desmond (1986) estabeleceu uma conexão entre a distribuição Gaussiana inversa e a distribuição Birnbaum-Saunders. Rieck e Nedelman (1991) propuseram um modelo log-linear para a distribuição Birnbaum-Saunders e desenvolveram métodos de estimação. Achcar e Espinosa (1991) desenvolveram métodos Bayesianos em testes de vida acelerados considerando um modelo loglinear para a distribuição Birnbaum-Saunders proposto por Rieck e Nedelman (1991). Lu e Chang (1997) utilizaram métodos bootstrap para construir intervalos de predição para a distribuição Birnbaum-Saunders. Dupuis e Mills (1998) propuseram métodos robustos de estimação dos parâmetros da distribuição Birnbaum-Saunders. Rieck (1999) derivou a função geradora de momentos para a distribuição seno hiperbólico normal que pode ser usada para obter momentos de ordem inteira ou fracionária da distribuição Birnbaum-Saunders. McCarter (1999) discutiu a estimação dos parâmetros da distribuição Birnbaum-Saunders para o caso de amostras censuradas do tipo II. Algumas generalizações e extensões da distribuição Birnbaum-Saunders foram propostas na última década. Owen e Padgett (1999) desenvolveram a distribuição Birnbaum-Saunders com três 
parâmetros. Recentemente, Díaz-García e Leiva (2005, 2007) propuseram uma nova classe de distribuições de vida generalizando a distribuição Birnbaum-Saunders a partir de distribuições de contornos elípticos. Segundo os autores, essa generalização baseia-se na busca de distribuições de vida que cresçam mais rapidamente e que possuam caudas com menor ou maior curtose do que a distribuição Birnbaum-Saunders, entre outras propriedades tais como: distribuições de vida que não possuem momentos e que sejam bimodais. É válido salientar que, neste caso, o uso de distribuições elípticas para gerar a distribuição Birnbaum-Saunders generalizada não segue uma justifitiva física, como no caso clássico (distribuição Birnbaum-Saunders baseada na distribuição normal), mas razões puramente estatísticas e/ou matemáticas. Além disso, a distribuição BirnbaumSaunders é um caso particular da distribuição Birnbaum-Saunders generalizada e muitas das propriedades da versão clássica são transferidas para a versão generalizada. Uma revisão recente dessa distribuição foi feita por Sanhueza et al. (2008) e uma aplicação da distribuição BirnbaumSaunders generalizada pode ser revisada no artigo de Leiva et al. (2008a), em que os autores usam a distribuição em um problema de contaminação do ar. Vilca-Labra e Leiva (2006), baseados em argumentos semelhantes aos empregados por Díaz-García e Leiva (2005, 2007), obtiveram uma outra generalização ao desenvolver a distribuição Birnbaum-Saunders mediante distribuições elípticas assimétricas. Assim, a distribuição Birnbaum-Saunders assimétrica, é mais flexível na curtose e assimetria do que todas as versões anteriores da distribuição Birnbaum-Saunders. Dois extensões interessantes são os artigos de Balakrishnan et al. (2009) e Guiraud et al. (2009). No primeiro, os autores consideram estimações na distribuição Birnbaum-Saunders baseadas em misturas de escala normal usando o algoritmo EM; e no segundo foi estudado uma versão não centrada da distribuição Birnbaum-Saunders. É importante destacar que as distribuições Birnbaum-Saunders e Birnbaum-Saunders generalizada encontram-se implementadas computacionalmente no software R (http://www.r-project.org) por meio dos pacotes bs (veja Leiva et al., 2006) e gbs (veja Barros et al., 2009), respectivamente.

\subsection{Derivação da distribuição Birnbaum-Saunders}

Birnbaum e Saunders (1969a) fizeram as seguintes suposições sobre o processo de fadiga:

1. Um material é submetido a um padrão cíclico de tensão e força;

2. A falha do material ocorre devido ao desenvolvimento e ao crescimento de uma fissura dominante no material, ou seja, quando o tamanho da fissura excede certo nível de resistência, denotado por $\omega$;

3. A sequência de tensão imposta ao material é a mesma ciclo a ciclo;

4. A extensão incremental da fissura $X_{i}$ resultante da aplicação da $i$-ésima oscilação de carga é uma variável aleatória com uma distribuição que só depende da fissura atual causada pela tensão neste ciclo; 
5. A extensão da fissura durante o $(j+1)$-ésimo ciclo é

$$
Y_{j+1}=X_{j m+1}+\ldots+X_{j m+i}+\ldots+X_{j m+m}, \text { para } j=0,1, \ldots
$$

em que $X_{j m+i}$ é a extensão da fissura (possivelmente microscópica) após a $i$-ésima oscilação de carga do $(j+1)$-ésimo ciclo;

6. As extensões das fissuras em diferentes ciclos são independentes;

7. A extensão total da fissura, $Y_{j}$, devido ao $j$-ésimo ciclo é uma variável aleatória que segue uma distribuição com média $\mu$ e variância $\sigma^{2}$, para $j=1,2, \ldots$.

Assim, a extensão total da fissura após $n$ ciclos é dada pela variável aleatória

$$
W_{n}=\sum_{j=1}^{n} Y_{j} .
$$

Segundo o teorema central do limite, na equação (1.1) e sob as suposições 6 e 7, é possível estabelecer para $n$ grande que

$$
W_{n} \quad \dot{\sim} \mathcal{N}\left(n \mu, n \sigma^{2}\right)
$$

ou seja, $W_{n}$ tem distribuição assintoticamente normal de média $n \mu$ e variância $n \sigma^{2}$.

Seja $N$ o número de ciclos requeridos até que seja observada a falha. A função de distribuição acumulada da variável aleatória $N$ é dada por

$$
\begin{aligned}
P(N \leq n) & =P\left(N \leq n, W_{n}>w\right)+P\left(N \leq n, W_{n} \leq \omega\right) \\
& =P\left(W_{n}>\omega\right)+P\left(N \leq n, W_{n} \leq \omega\right) .
\end{aligned}
$$

Note que

$$
P\left(N \leq n, W_{n} \leq \omega\right)>0
$$

já que $W_{n}$ assintoticamente segue uma distribuição normal. Birnbaum e Saunders (1969a) consideraram que o termo presente na equação (1.2) pode ser desprezado e usaram a aproximação

$$
P(N \leq n) \approx P\left(W_{n}>\omega\right)
$$


Padronizando os termos da equação (1.3), temos que

$$
\begin{aligned}
P(N \leq n) & \approx P\left(\frac{W_{n}-n \mu}{\sigma \sqrt{n}}>\frac{\omega-n \mu}{\sigma \sqrt{n}}\right) \\
& =P\left(\frac{W_{n}-n \mu}{\sigma \sqrt{n}} \leq \frac{n \mu-\omega}{\sigma \sqrt{n}}\right) \\
& =\Phi\left(\frac{n \mu-\omega}{\sigma \sqrt{n}}\right) \\
& =\Phi\left(\frac{\sqrt{\omega \mu}}{\sigma}\left[\sqrt{\frac{n}{\omega / \mu}}-\sqrt{\frac{\omega / \mu}{n}}\right]\right),
\end{aligned}
$$

em que $\Phi(\cdot)$ é a função de distribuição acumulada de uma variável aleatória $\mathcal{N}(0,1)$.

Birnbaum e Saunders (1969a) utilizaram a equação (1.4) para definir uma distribuição contínua de vida. De acordo com os autores, se $n$ for substituído por uma variável aleatória real não negativa $t$, então a variável aleatória $T$ é a extensão contínua da variável aleatória discreta $N$. Desta forma, $T$ representa o tempo total até que ocorra uma falha. Assim, tomando

$$
\alpha=\frac{\sigma}{\sqrt{\mu \omega}} \quad \text { e } \quad \beta=\frac{\omega}{\mu}
$$

a função de distribuição acumulada de $T$ pode ser escrita como

$$
F_{T}(t)=P(T \leq t)=\Phi\left(\frac{1}{\alpha}\left[\sqrt{\frac{t}{\beta}}-\sqrt{\frac{\beta}{t}}\right]\right), \quad t>0,
$$

em que $\alpha>0$ e $\beta>0$. Dizemos, então, que $T$ segue uma distribuição Birnbaum-Saunders com parâmetros $\alpha$ e $\beta$ e denotamos por $T \sim \mathcal{B S}(\alpha, \beta)$. A seguinte seção tem como objetivo apresentar as distribuições simétricas univariadas que foram usadas por Díaz-García e Leiva $(2005,2007)$ para estender a distribuição Birnbaum-Saunders e propor uma nova classe de distribuições mais flexíveis. Díaz-García e Leiva (2005, 2007) denomiram essa classe de distribuições BirnbaumSaunders generalizada as quais serão estudadas com mais detalhes no Capítulo 2.

\subsection{Distribuição simétrica univariada}

Definição 1. (Distribuição simétrica univariada)

Uma variável aleatória tem distribuição simétrica univariada com parâmetros de posição $\mu$, escala $\sigma^{2}$ e função geradora de densidades $g$, se sua função densidade de probabilidade pode ser escrita como (Fang et al., 1990)

$$
f_{Y}(y)=\frac{1}{\sqrt{\sigma^{2}}} g\left(\frac{[y-\mu]^{2}}{\sigma^{2}}\right), y \in \mathbb{R}, \mu \in \mathbb{R}, \sigma^{2} \in \mathbb{R}^{+},
$$


em que a função $g: \mathbb{R} \rightarrow \mathbb{R}^{+}$satisfaz a condição

$$
\int_{0}^{+\infty} u^{-1 / 2} g(u) \mathrm{d} u<\infty
$$

$\operatorname{com} u=\frac{[y-\mu]^{2}}{\sigma^{2}}$. Notação $Y \sim \mathcal{S}\left(\mu, \sigma^{2} ; g\right)$.

Um caso particular da distribuição (1.6) é obtido quando $\mu=0$ e $\sigma^{2}=1$, em cujo caso se diz que $Z \sim \mathcal{S}(0,1 ; g)$ possui uma distribuição simétrica padrão com função geradora de densidades $g$, cuja função densidade de probabilidade é dada por

$$
f_{Z}(z)=g\left(z^{2}\right), \quad z \in \mathbb{R}
$$

Algumas distribuições simétricas padrão são apresentadas na Tabela 1.1.

Tabela 1.1: Função densidade de probabilidade para algumas distribuições simétricas padrão.

\section{Distribuição Função densidade de probabilidade}

$$
\begin{array}{ll}
\text { Normal } & f_{Z}(z)=\frac{1}{\sqrt{2 \pi}} \exp \left(-\frac{z^{2}}{2}\right) \\
\text { Cauchy } & f_{Z}(z)=\frac{1}{\pi}\left(\frac{1}{1+z^{2}}\right) \\
\text { Laplace } & f_{Z}(z)=\frac{1}{4} \exp \left(-\frac{z}{2}\right) \\
\text { t-Student } & f_{Z}(z)=\frac{\Gamma([\nu+1] / 2)}{\Gamma(\nu / 2) \sqrt{\nu \pi}}\left(1+\frac{z^{2}}{\nu}\right)^{-(\nu+1) / 2}, \nu>0 \\
\text { Exponencial potência } & f_{Z}(z)=\frac{2^{-(1+1 / 2 \nu)}}{\Gamma(1+1 / 2 \nu)} \exp \left(-\frac{z^{2 \nu}}{2}\right), \quad \nu \geq \frac{1}{2} \\
\text { Kotz } & f_{Z}(z)=\frac{\nu r^{(2 q-1) /(2 \nu)}}{\Gamma([2 q-1] / 2 \nu)} z^{2(q-1)} \exp \left(-r z^{2 \nu}\right) \quad r, \nu>0, q \geq 1 / 2
\end{array}
$$


Observação 1. Algumas distribuições simétricas estão relacionadas da seguinte forma:

(i) $t$-Student $\left(\mu, \sigma^{2}, \nu=1\right) \equiv \operatorname{Cauchy}\left(\mu, \sigma^{2}\right)$.

(ii) Exponencial potência $\left(\mu, \sigma^{2}, \nu=1\right) \equiv \mathcal{N}\left(\mu, \sigma^{2}\right)$.

(iii) Exponencial potência $\left(\mu, \sigma^{2}, \nu=1 / 2\right) \equiv \operatorname{Laplace}\left(\mu, \sigma^{2}\right)$.

(iv) $\operatorname{Kotz}\left(\mu, \sigma^{2}, \nu=1, r=1 / 2, q=1\right) \equiv \mathcal{N}\left(\mu, \sigma^{2}\right)$.

Para mais detalhes das distribuições apresentadas na Tabela 1.1, pode-se consultar, por exemplo, Ferrari e Uribe-Opazo (2001), Cordeiro et al. (2000) e Johnson et al. (1994, 1995).

Com base no trabalho de Rieck e Nedelman (1991) e utilizando as distribuições simétricas univariadas, Díaz-García e Domínguez-Molina (2006) criaram uma distribuição chamada seno hiperbólico simétrica a qual tem como casos particulares a distribuição seno hiperbólico normal e seno hiperbólico t que detalhamos a seguir.

\subsection{Distribuição seno hiperbólico simétrica}

Definição 2. (Distribuição seno hiperbólico simétrica)

A função densidade de probabilidade de uma variável aleatória $Y$ com distribuição seno hiperbólico simétrica, com parâmetro de forma $\alpha$, parâmetro de posição $\gamma$, parâmetro de escala $\sigma$ e função geradora de densidades g é definida por (veja, Díaz-García e Domínguez-Molina, 2006)

$$
f_{Y}(y)=f_{Z}\left(b_{y}\right) B_{y}
$$

em que $Z \sim \mathcal{S}(0,1 ; g)$,

$$
b_{y}=b_{y}(\alpha, \gamma, \sigma)=\frac{2}{\alpha} \operatorname{senh}\left(\frac{y-\gamma}{\sigma}\right), \quad B_{y}=\frac{d}{d y} b_{y}=\frac{2}{\alpha \sigma} \cosh \left(\frac{y-\gamma}{\sigma}\right),
$$

$\alpha>0, \gamma \in \mathbb{R}$ e $\sigma>0$. Notação: $Y \sim \mathcal{S H} \mathcal{H}(\alpha, \gamma, \sigma ; g)$.

\subsection{Distribuição seno hiperbólico normal}

Nesta seção definimos a distribuição seno hiperbólico normal, apresentamos algumas propriedades e estudamos o comportameno gráfico da distribuição.

Definição 3. (Distribuição seno hiperbólico normal)

A função densidade de probabilidade de uma variável aleatória $Y$ com distribuição seno hiperbólico normal, com parâmetro de forma $\alpha$, parâmetro de posição $\gamma$ e parâmetro de escala $\sigma$ é definida por (Rieck e Nedelman, 1991)

$$
f_{Y}(y)=\phi\left(\frac{2}{\alpha} \operatorname{senh}\left(\frac{y-\gamma}{\sigma}\right)\right) \frac{2}{\alpha \sigma} \cosh \left(\frac{y-\gamma}{\sigma}\right), \quad y \in \mathbb{R}
$$

em que $\phi(\cdot)$ representa a função de distribuição de probabilidade da distribuição normal padrão, $\alpha>0, \gamma \in \mathbb{R} e \sigma>0$. Notação: $Y \sim \mathcal{S H \mathcal { N }}(\alpha, \gamma, \sigma)$. 
Observação 2. A distribuição seno hiperbólico normal, desenvolvida por Rieck e Nedelman (1991), pode ser definida através de uma transformação de uma distribuição normal padrão por meio da variável aleatória $Y=\operatorname{arcsenh}(\alpha Z / 2) \sigma+\gamma, \alpha>0, \gamma \in \mathbb{R} e \sigma>0$, em que $Z \sim \mathcal{N}(0,1)$.

\subsubsection{Propriedades e análise gráfica}

Se $Y \sim \mathcal{S H \mathcal { N }}(\alpha, \gamma, \sigma)$, então o valor esperado e a variância são definidas como (Rieck e Nedelman, 1991):

$$
\begin{aligned}
\mathrm{E}[Y] & =\gamma \mathrm{e} \\
\operatorname{Var}[Y] & =\sigma^{2} \omega(\alpha),
\end{aligned}
$$

em que $\omega(\alpha)$ é definido como

$$
\omega(\alpha)=\mathrm{E}\left[S^{2}\right]=\int_{-\infty}^{+\infty} s^{2} \frac{2 \cosh (s)}{\sqrt{2 \pi \alpha^{2}}} \exp \left\{-\frac{2}{\alpha^{2}} \operatorname{senh}^{2} s\right\} \mathrm{d} s
$$

$S \sim \mathcal{S H \mathcal { N }}(\alpha, 0,1)$. E[ $\left.S^{2}\right]$ é a variância quando $\sigma=1$. Se $Y \sim \mathcal{S H \mathcal { N }}(\alpha, \gamma, \sigma)$, então a função de distribuição acumulada de $Y$ é dada por

$$
F_{Y}(y)=\Phi\left(\frac{2}{\alpha} \operatorname{senh}\left(\frac{y-\gamma}{\sigma}\right)\right)
$$

Rieck e Nedelman (1991) mostraram que a distribuição seno hiperbólico normal é simétrica em torno de $\gamma$, unimodal para $\alpha \leq 2$ e bimodal para $\alpha>2$ como podemos observar na Figura 1.2. $\mathrm{O}$ código para gerar a Figura 1.2, baseado no pacote bs do R (Leiva et al., 2006), é apresentado no Apêndice A.

\subsubsection{Função geradora de momentos}

A função geradora de momentos para uma distribuição $\mathcal{S H \mathcal { N }}(\alpha, \gamma, \sigma)$ é dada por

$$
m_{Y}(s)=\exp (\gamma s)\left[\frac{K_{a}\left(\alpha^{-2}\right)+K_{b}\left(\alpha^{-2}\right)}{2 K_{1 / 2}\left(\alpha^{-2}\right)}\right],
$$

em que $a=(\sigma s+1) / 2, b=(\sigma s-1) / 2$ e $K_{\lambda}(\cdot)$ é a função de bessel modificada do terceiro tipo (veja Gradshteyn e Ryzhik, 2007, página 917), dada por

$$
K_{\lambda}(w)=(1 / 2)(w / 2)^{\lambda} \int_{0}^{\infty} y^{-\lambda-1} e^{-y-\left(w^{2} / 4 y\right)} d y .
$$

Assim,

$$
K_{\frac{1}{2}}(w)=\sqrt{\frac{\pi}{2 w}} \exp (-w), \quad K_{\frac{3}{2}}(w)=\sqrt{\frac{\pi}{2 w}} \exp (-w)\left[1+\frac{1}{w}\right]
$$




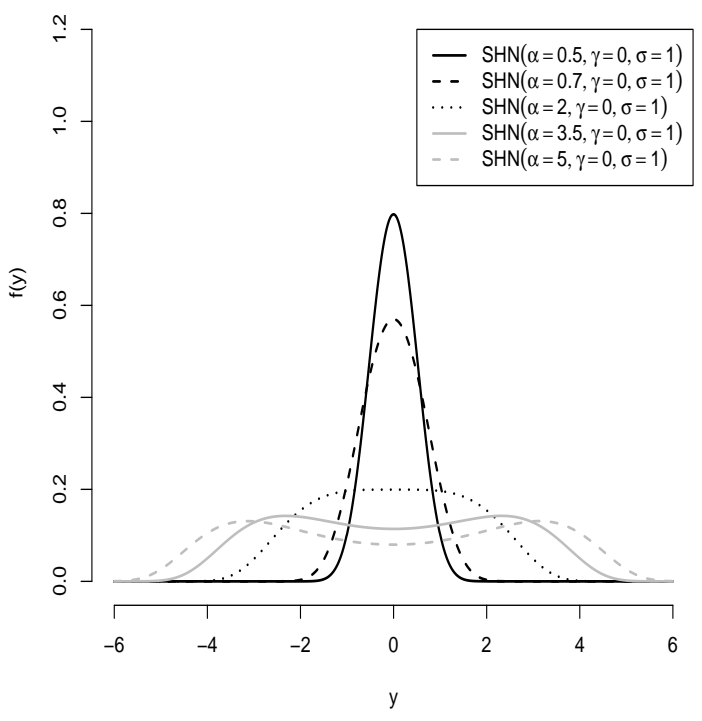

Figura 1.2: Gráfico da função densidade de probabilidade SHN para $\gamma=0, \sigma=1$ e diferentes valores de $\alpha$.

e

$$
K_{\frac{5}{2}}(w)=\sqrt{\frac{\pi}{2 w}} \exp (-v)\left[1+\frac{3}{w}+\frac{3}{w^{2}}\right]
$$

Algumas aproximações da função de bessel modificada do terceiro tipo são

$$
K_{0}(w) \approx \log (w) \quad \text { e } \quad K_{m}(w) \approx \frac{(m-1) !}{2}\left[\frac{w}{2}\right]^{-m} .
$$

Com base na equação (1.13), pode-se obter a média e a variância da distribuição $\mathcal{S H \mathcal { N }}(\alpha, \gamma, \sigma)$. A função de sobrevivência e a função de risco para uma variável aleatória $Y \sim \mathcal{S H \mathcal { N }}(\alpha, \gamma, \sigma)$ são dadas por

$$
\begin{aligned}
S_{Y}(y) & =1-\Phi\left(b_{y}\right) \mathrm{e} \\
h_{Y}(y) & =\frac{f_{Y}(y)}{S_{Y}(y)}=\frac{\phi\left(b_{y}\right) B_{y}}{1-\Phi\left(b_{y}\right)},
\end{aligned}
$$

em que $\phi(\cdot)$ e $\Phi(\cdot)$ são, respectivamente, a função densidade de probabilidade e função de distribuição acumulada de uma distribuição normal padrão, $b_{y}$ e $B_{Y}$ são dados na equação (1.9). 


\subsection{Distribuição seno hiperbólico $\mathbf{t}$}

Usaremos a notação $\operatorname{SHt}(\alpha, \gamma, \sigma ; \nu)$ para representar que a variável aleatória $Y$ possui uma distribuição seno hiperbólico simétrica com parâmetro de forma $\alpha$, parâmetro de posição $\gamma$, parâmetro de escala $\sigma$ e função geradora de densidades $g$, correspondente a uma distribuição t-Student padrão com $\nu$ graus de liberdade. Portanto, a função densidade de probabilidade da variável aleatória $Y \sim \operatorname{SHt}(\alpha, \gamma, \sigma ; \nu)$ é dada por

$$
f_{Y}(y)=\phi_{t}\left(\frac{2}{\alpha} \operatorname{senh}\left(\frac{y-\gamma}{\sigma}\right)\right) \frac{2}{\alpha \sigma} \cosh \left(\frac{y-\gamma}{\sigma}\right),
$$

em que

$$
\phi_{t}\left(\frac{2}{\alpha} \operatorname{senh}\left(\frac{y-\gamma}{\sigma}\right)\right)=\frac{\Gamma([\nu+1] / 2)}{\Gamma(\nu / 2) \sqrt{\nu \pi}}\left[1+\frac{4}{\nu \alpha^{2}} \operatorname{senh}^{2}\left(\frac{y-\gamma}{\sigma}\right)\right]^{-[\nu+1] / 2}
$$

e a função de distribuição acumulada é dada por

$$
F_{Y}(y)=\Phi_{t}\left(\frac{2}{\alpha} \operatorname{senh}\left(\frac{y-\gamma}{\sigma}\right)\right)=\frac{1}{2}\left[1+I_{\frac{\xi^{2}}{\xi^{2}+\nu}}\left(\frac{1}{2}, \frac{\nu}{2}\right)\right],
$$

em que

$$
\xi=\frac{2}{\alpha \sigma} \operatorname{senh}\left(\frac{y-\gamma}{\sigma}\right), \quad I_{x}(a, b)=\frac{\int_{0}^{x} t^{a-1}(1-t)^{b-1} \mathrm{~d} t}{\int_{0}^{1} t^{a-1}(1-t)^{b-1} \mathrm{~d} t}
$$

é a razão da função beta incompleta e $\Phi_{t}(\cdot)$ é a função de distribuição acumulada de uma variável aleatória t-Student padrão $\operatorname{com} \nu$ graus de liberdade. Em resumo, podemos escrever a função densidade de probabilidade de $Y \sim \operatorname{SHt}(\alpha, \gamma, \sigma ; \nu)$ como

$$
f_{Y}(y)=\frac{\Gamma([\nu+1] / 2)}{\Gamma(\nu / 2) \sqrt{\nu \pi}}\left[1+\frac{4}{\nu \alpha^{2}} \operatorname{senh}^{2}\left(\frac{y-\gamma}{\sigma}\right)\right]^{-[\nu+1] / 2} \frac{2}{\alpha \sigma} \cosh \left(\frac{y-\gamma}{\sigma}\right) .
$$

A função de risco é definida por

$$
h_{Y}(y)=\frac{\phi_{t}\left(b_{y}\right) B_{y}}{1-\Phi_{t}\left(B_{y}\right)}
$$

com $b_{y}$ e $B_{y}$ dadas na equação (1.9).

\subsection{Estrutura do trabalho}

A presente tese de doutorado tem como objetivo principal estudar os modelos Birnbaum-Saunders com efeitos mistos. A tese é constituída de 7 capítulos descritos a seguir:

* Capítulo 1: apresentamos alguns conceitos básicos na análise de sobrevivência. Fazemos uma revisão da literatura e mostramos a derivação da distribuição Birnbaum-Saunders. Finalmente apresentamos as distribuições simétricas univariadas, seno hiperbólico simétrica, seno hiperbólico normal, seno hiperbólico t e a estrutura da tese. 
* Capítulo 2: apresentamos uma revisão da distribuição Birnbaum-Saunders e BirnbaumSaunders generalizada, mostrando suas propriedades e realizando análises gráficas.

* Capítulo 3: apresentamos um resumo de alguns resultados para os modelos log-BirnbaumSaunders e log-Birnbaum-Saunders-t de efeitos fixos. Apresentamos o método de influência local e o resíduo tipo martingale.

* Capítulos 4 e 5: apresentamos os modelos log-Birnbaum-Saunders e log-Birnbaum-Saunders-t de efeitos aleatórios, calculamos a função escore e a matriz hessiana baseadas na quadratura de Gauss-Hermite. São apresentadas expressões para a esperança, variância, covariância e correlação para esses modelos. Finalmente, estudamos em particular, os modelos log-Birnbaum-Saunders e log-Birnbaum-Saunders-t de intercepto aleatório.

* Capítulo 6: aplicamos a um conjunto de dados reais os modelos log-Birnbaum-Saunders e log-Birnbaum-Saunders-t de intercepto aleatório.

* Capítulo 7: apresentamos as conclusões com os principais resultados e contribuições desta tese. Serão também apresentadas algumas direções para pesquisas futuras. 



\section{Capítulo 2}

\section{Distribuições Birnbaum-Saunders e Birnbaum-Saunders generalizada}

A distribuição Birnbaum-Saunders generalizada (BSG) foi proposta por Díaz-García e Leiva (2005, 2007), baseando-se em distribuições simétricas univariadas (ou elípticas univariadas). Diversos autores têm estudado as distribuições elípticas (multivariadas e univariadas), tais como, Kelker (1970), Dawid (1977), Cambanis et al. (1981), Anderson e Fang (1987) e Fang et al. (1990), entre outros.

Neste capítulo, descrevemos a distribuição Birnbaum-Saunders (veja, por exemplo Birnbaum e Saunders, 1969a,b) e geramos a distribuição Birnbaum-Saunders generalizada assumindo distribuições simétricas padrão, isto é, a normal, Cauchy, Laplace, t-Student, exponencial potência e Kotz que foram estudadas no Capítulo 1. Algumas propriedades e representações gráficas são apresentadas para ilustrar as particularidades e flexibilidades destas distribuições. O objetivo principal das distribuições Birnbaum-Saunders generalizadas é ter uma classe de distribuições que possam acomodar melhor as observações atípicas, isto é, ter distribuições com caudas mais leves e mais pesadas do que a distribuição Birnbaum-Saunders. Cabe destacar que a distribuição Birnbaum-Saunders é um caso particular da distribuição Birnbaum-Saunders generalizada, ou seja, quando assumimos uma distribuição normal padrão.

Finalmennte, relacionamos as distribuições Birnbaum-Saunders generalizada e seno hiperbólico simétrica com a distribuição log-Birnbaum-Saunders generalizada (log-BSG) que tem como casos particulares as distribuições log-Birnbaum-Saunders (log-BS) e log-Birnbaum-Saunders-t (log-BSt), que serão usadas no Capítulo 3. 


\subsection{Distribuição Birnbaum-Saunders}

Definição 4. (Distribuição Birnbaum-Saunders)

A função densidade de probabilidade de uma variável aleatória $T$ que segue uma distribuição Birnbaum-Saunders, com parâmetro de forma $\alpha$ e parâmetro de escala $\beta$ (também correspondente à mediana) é definida por

$$
f_{T}(t)=\phi\left(a_{t}\right) A_{t}, \quad t>0
$$

em que $\phi(\cdot)$ representa a função de distribuição de probabilidade da distribuição normal padrão,

$$
a_{t}=a_{t}(\alpha, \beta)=\frac{1}{\alpha}\left[\sqrt{\frac{t}{\beta}}-\sqrt{\frac{\beta}{t}}\right], \quad A_{t}=\frac{d}{d t} a_{t}=\frac{t^{-3 / 2}(t+\beta)}{2 \alpha \sqrt{\beta}}
$$

com $\alpha>0$ e $\beta>0$. Notação: $T \sim \mathcal{B S}(\alpha, \beta)$.

Temos que $T \sim \mathcal{B S}(\alpha, \beta)$ é definida em termos da distribuição normal por meio da variável aleatória

$$
T=\beta\left[\frac{\alpha Z}{2}+\sqrt{\left(\frac{\alpha Z}{2}\right)^{2}+1}\right]^{2},
$$

em que $Z \sim \mathcal{N}(0,1)$. Assim, a variável aleatória $Z$ pode ser representada estocasticamente em termos de $T$ escrevendo

$$
Z=\frac{1}{\alpha}\left[\sqrt{\frac{T}{\beta}}-\sqrt{\frac{\beta}{T}}\right] \sim \mathcal{N}(0,1) .
$$

Se $T \sim \mathcal{B S}(\alpha, \beta)$, então a função de distribuição acumulada de $T$ é dada por

$$
F_{T}(t)=\Phi\left(\frac{1}{\alpha}\left[\sqrt{\frac{t}{\beta}}-\sqrt{\frac{\beta}{t}}\right]\right) .
$$

\subsubsection{Propriedades e análise gráfica}

Pode se provar que se $T \sim \mathcal{B S}(\alpha, \beta)$, então segue que

$$
\mathrm{E}[T]=\beta\left(1+\frac{\alpha^{2}}{2}\right) \quad \text { e } \operatorname{Var}[T]=\beta^{2} \alpha^{2}\left(\frac{5}{4} \alpha^{2}+1\right)
$$

Duas propriedades interessantes da distribuição Birnbaum-Saunders são as seguintes:

1. Se $c>0$, então $c T \sim \mathcal{B S}(\alpha, c \beta)$.

2. Se $T \sim \mathcal{B S}(\alpha, \beta)$, então a variável aleatória $T^{-1} \sim \mathcal{B S}\left(\alpha, \beta^{-1}\right)$, isto é, a distribuição Birnbaum-Saunders possui a propriedade de ser fechada sob reciprocidade (Saunders, 1974). 
A função de sobrevivência e a função de risco da distribuição Birnbaum-Saunders são dadas, respectivamente, por

$$
\begin{aligned}
S_{T}(t) & =1-\Phi\left(a_{t}\right) \mathrm{e} \\
h_{T}(t) & =\frac{f_{T}(t)}{S_{T}(t)}=\frac{\phi\left(a_{t}\right) A_{t}}{1-\Phi\left(a_{t}\right)},
\end{aligned}
$$

em que $\phi(\cdot)$ e $\Phi(\cdot)$ representam a função densidade de probabilidade e função de distribuição acumulada da distribuição normal padrão, $a_{t}$ e $A_{t}$ são dados em (2.2).

Observação 3. A distribuição Birnbaum-Saunders torna-se assimétrica à medida que $\alpha$ cresce, $e$ simétrica, em torno de $\beta$, à medida que $\alpha$ se aproxima de zero. Este comportamento pode ser visto na Figura 2.1 (esquerda). A função de risco da variável aleatória $T \sim \mathcal{B S}(\alpha, \beta)$, assume valor zero em $t=0$, cresce até um valor máximo e depois decresce até uma constante positiva, como podemos observar na Figura 2.1 (direita).
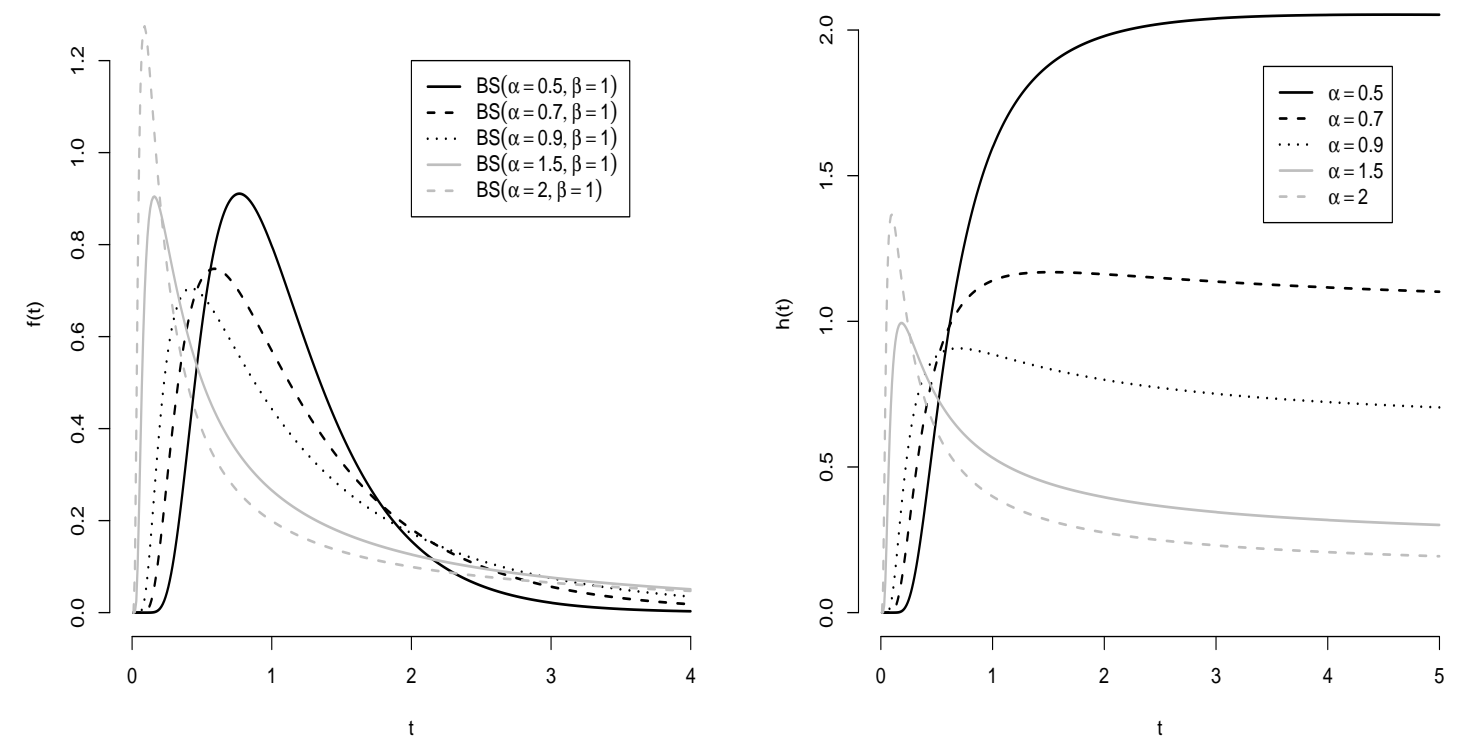

Figura 2.1: Gráfico da função densidade de probabilidade (esquerda) e função de risco (direita) da distribuição BS para $\beta=1$ e diferentes valores de $\alpha$.

Analogamente à seção anterior, apresentamos algumas propriedades e gráficos da distribuição Birnbaum-Saunders generalizada. 


\subsection{Distribuição Birnbaum-Saunders generalizada}

Definição 5. (Distribuição Birnbaum-Saunders generalizada)

A função densidade de probabilidade de uma variável aleatória $T$ com distribuição Birnbaum-Saunders generalizada, com parâmetro de forma $\alpha$, parâmetro de escala $\beta$ (também correspondente à mediana) e função geradora de densidades $g$ é definida por

$$
f_{T}(t)=f_{Z}\left(a_{t}\right) A_{t}
$$

em que $a_{t}$ e $A_{t}$ são definidas na equação (2.1). Notação: $T \sim \mathcal{B S G}(\alpha, \beta ; g)$.

Temos que $T \sim \mathcal{B S G}(\alpha, \beta ; g)$ é definida em termos da distribuição simétrica univariada por meio da variável aleatória

$$
T=\beta\left[\frac{\alpha Z}{2}+\sqrt{\left(\frac{\alpha Z}{2}\right)^{2}+1}\right]^{2},
$$

em que $Z \sim \mathcal{S}(0,1 ; g)$. Assim, a variável aleatória $Z$ pode ser representada estocasticamente em termos de $T$ como

$$
Z=\frac{1}{\alpha}\left[\sqrt{\frac{T}{\beta}}-\sqrt{\frac{\beta}{T}}\right] \sim \mathcal{S}(0,1 ; g) .
$$

Logo, se $T \sim \mathcal{B S G}(\alpha, \beta ; g)$, então a função de distribuição acumulada de $T$ pode ser escrita como

$$
F_{T}(t)=F_{Z}\left(a_{t}\right), t>0
$$

A(s) moda(s) de $T \sim \mathcal{B S G}(\alpha, \beta ; g)$, denotada(s) por $t_{m}$, é (são) resultante(s) da(s) solução(ões)

$$
\zeta_{g}\left(a_{t_{m}}^{2}\right)=\frac{\alpha^{2} \beta t_{m}\left[t_{m}+3 \beta\right]}{2\left[t_{m}-\beta\right]\left[t_{m}+\beta\right]^{2}}
$$

em que $\zeta_{g}(\cdot)$ é dado por

$$
\zeta_{g}(u)=\frac{g^{\prime}(u)}{g(u)}, \quad u>0 .
$$

Nesse caso $g^{\prime}(\cdot)$ denota a derivada de $g(\cdot)$ dada na equação (1.6). O $q$-ésimo percentil de $T$ é dado por

$$
t(q)=F_{T}^{-1}(q)=\frac{\beta}{4}\left[\alpha z_{q}+\sqrt{\alpha^{2} z_{q}^{2}+4}\right]^{2} ; \quad 0<q \leq 1,
$$

em que $z_{q}$ é o $q$-ésimo percentil de $Z \sim \mathcal{S}(0,1 ; g)$ e $F_{T}^{-1}(\cdot)$ é a inversa da função de distribuição acumulada da variável aleatória $T$. 


\subsubsection{Propriedades e análise gráfica}

Algumas propriedades da distribuição Birnbaum-Saunders generalizada são as seguintes (veja Sanhueza et al., 2008):

(1) Se $T \sim \mathcal{B S G}(\alpha, \beta ; g)$, então para $a>0 ; a T \sim \mathcal{B S G}(\alpha, a \beta ; g)$.

(2) Se $T \sim \mathcal{B S} \mathcal{G}(\alpha, \beta ; g)$, então a variável aleatória $T^{-1} \sim \mathcal{B S} \mathcal{G}\left(\alpha, \beta^{-1} ; g\right)$, isto é, a distribuição Birnbaum-Saunders generalizada é fechada sob reciprocidade; veja Saunders (1974).

As diferentes formas da distribuição Birnbaum-Saunders generalizada, geradas a partir de diferentes distribuições simétricas padrão, são apresentadas nas Figuras 2.2 e 2.3. A Figura 2.2 (esquerda) ilustra uma distribuição bimodal que não possui momentos, gerada pela distribuição Cauchy; e a Figura 2.2 (direita) mostra que quando o parâmetro $\nu$ cresce, a distribuição tende à distribuição Birnbaum-Saunders clássica, ou seja, gerada pela distribuição normal. A Figura 2.3 (esquerda) ilustra uma distribuição com caudas mais leves que a distribuição Birnbaum-Saunders clássica; gerada pela distribuição exponencial potência; e a Figura 2.3 (direita) ilustra uma distribuição bimodal (gerada pela distribuição Kotz).
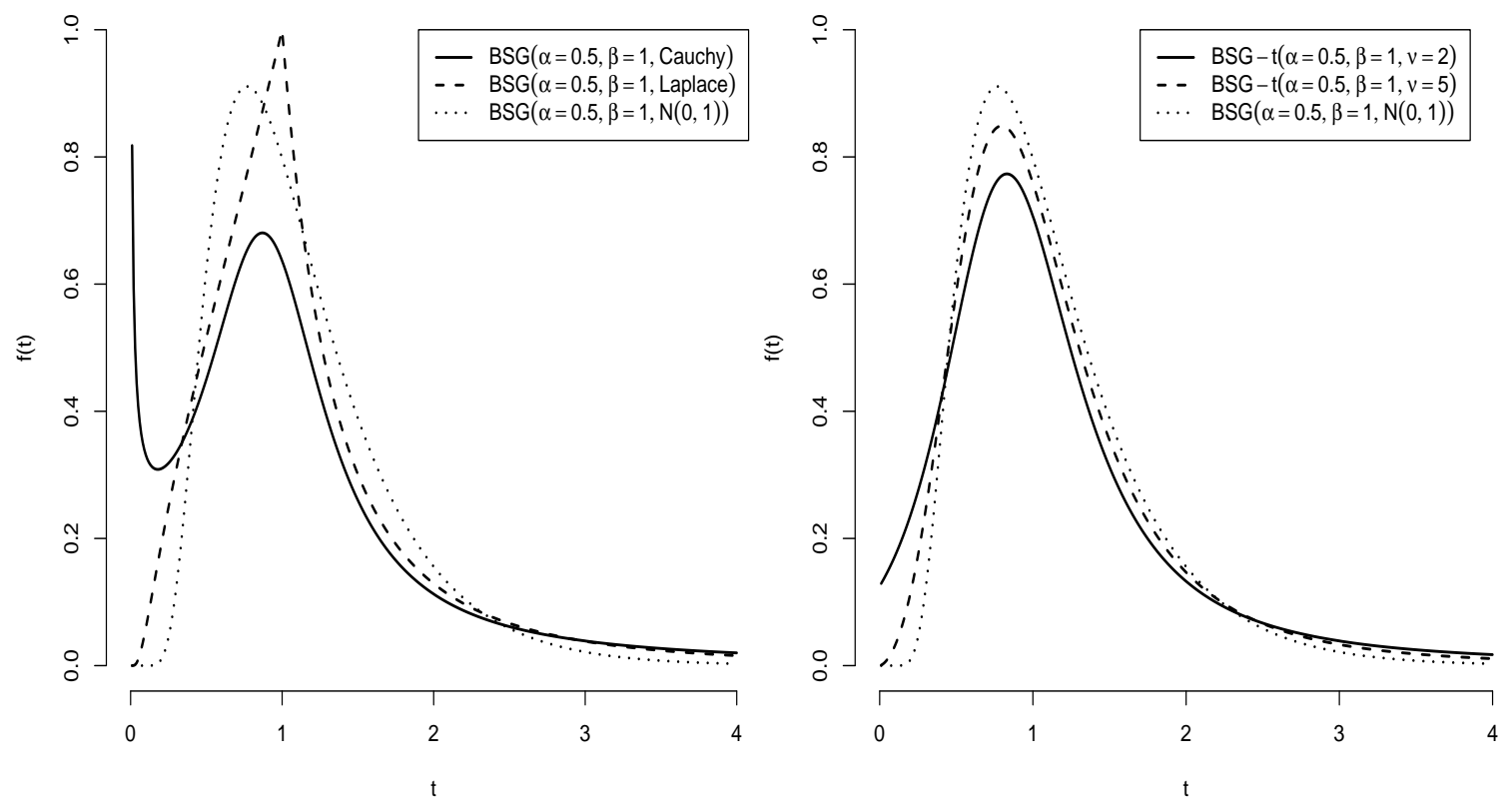

Figura 2.2: Gráfico da distribuição BSG para $\alpha=0,5 ; \beta=1$ obtidas a partir das distribuições Cauchy, Laplace, normal (esquerda); t-Student $\operatorname{com} \nu=2$ e $\nu=5$ graus de liberdade e normal (direita). 

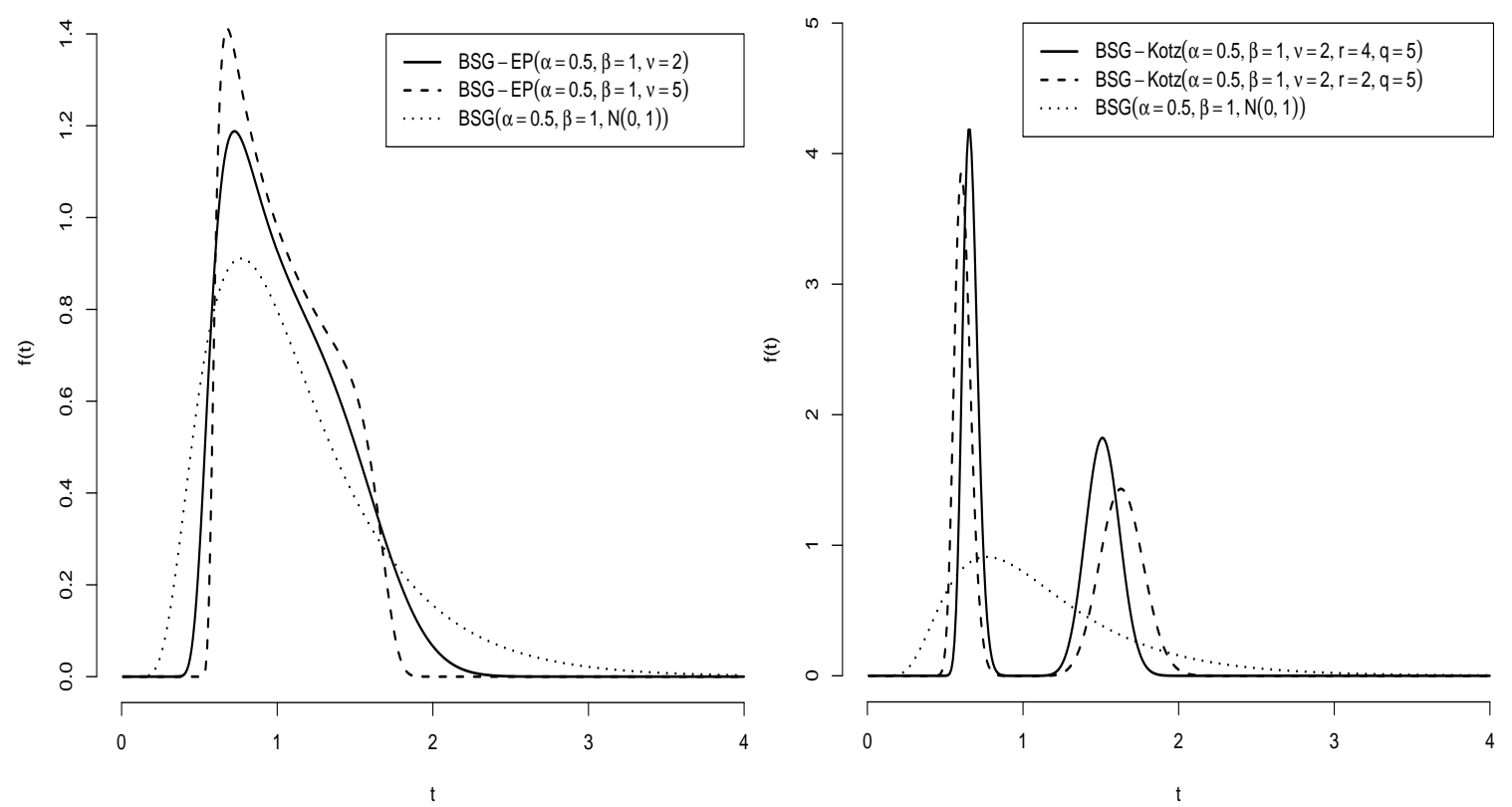

Figura 2.3: Gráfico da distribuição BSG para $\alpha=0,5 ; \beta=1$ obtidas a partir das distribuições exponencial potência com $\nu=2$ e $\nu=5$ graus de liberdade e normal (esquerda); $\operatorname{Kotz}(\nu=2, \mathrm{r}=4, \mathrm{q}=5), \operatorname{Kotz}(\nu=2$, $\mathrm{r}=2, \mathrm{q}=5$ ) e normal (direita).

\subsubsection{Momentos}

Se $Z \sim \mathcal{S}(0,1 ; g), U=Z^{2} \sim \mathcal{G} \chi^{2}(1 ; g)$ (qui-quadrado generalizada) e se $\mathrm{E}\left[Z^{k}\right]$ existe, então

$$
\mathrm{E}\left[T^{k}\right]=\beta^{k} \sum_{i=0}^{k}\left(\begin{array}{l}
2 k \\
2 i
\end{array}\right) \sum_{j=0}^{i}\left(\begin{array}{l}
i \\
j
\end{array}\right) \mathrm{E}\left[U^{k+j-i}\right]\left[\frac{\alpha}{2}\right]^{2[k+j-i]}, \quad k=1,2, \ldots
$$

Das propriedades (1) e (2) (Seção 2.2.1), os momentos negativos de $T$ podem ser obtidos usando o fato, de que $\beta / T$ e $T / \beta$ têm a mesma distribuição, isto é,

$$
\frac{\beta}{T} \stackrel{\mathrm{d}}{=} \frac{T}{\beta} \sim \mathcal{B S G}(\alpha, 1 ; g)
$$

Portanto,

$$
\mathrm{E}\left[T^{-k}\right]=\frac{\mathrm{E}\left[T^{k}\right]}{\beta^{2 k}}, \quad k=1,2, \ldots
$$

Uma alternativa para calcular o $k$-ésimo momento da distribuição Birnbaum-Saunders generalizada, ao invés da equação (2.14), é

$$
\mathrm{E}\left[T^{k}\right]=\frac{\beta^{k} u_{k}}{2 \alpha^{-2 k}}-\frac{1}{2}\left[\sum_{j=1}^{k}\left(\begin{array}{c}
2 k \\
j
\end{array}\right) \frac{\mathrm{E}\left[T^{k-j}\right]}{\beta^{-j}[-1]^{j}}+\sum_{j=k+1}^{2 k-1}\left(\begin{array}{c}
2 k \\
j
\end{array}\right) \frac{\mathrm{E}\left[T^{j-k}\right]}{\beta^{j-2 k}[-1]^{j}}\right],
$$


para $k \in \mathbb{N}$, em que $u_{k}=\mathrm{E}\left[U^{k}\right]$. Das equações (2.14) ou (2.15), o primeiro momento não central de $T$ é dado por $\mathrm{E}[T]=\frac{\beta}{2}\left[2+u_{1} \alpha^{2}\right]$ e o segundo momento central de $T$, isto é, $\operatorname{Var}[T]=$ $\mathrm{E}\left[T^{2}\right]-\mathrm{E}^{2}[T]=\frac{\beta^{2} \alpha^{2}}{4}\left[4 u_{1}+\left[2 u_{2}-u_{1}^{2}\right] \alpha^{2}\right]$.

A função de sobrevivência e função de risco de uma variável aleatória $T \sim \mathcal{B S G}(\alpha, \beta ; g)$ são, respectivamente, dados por

$$
\begin{aligned}
S_{T}(t) & =1-F_{Z}\left(a_{t}\right) \mathrm{e} \\
h_{T}(t) & =\frac{f_{T}(t)}{S_{T}(t)}=\frac{f_{Z}\left(a_{t}\right) A_{t}}{1-F_{Z}\left(a_{t}\right)},
\end{aligned}
$$

em que $Z \sim \mathcal{S}(0,1 ; g)$, a $a_{t}$ e $A_{t}$ são definidas na equação (2.1). A seguir apresentamos alguns exemplos de distribuições Birnbaum-Saunders generalizadas, baseados na Tabela 1.1.

Exemplo 1. Se $T \sim \mathcal{B S G}(\alpha, \beta ; g)$, então para uma dada função geradora de densidades $g$ especificada em parênteses colchetes, a função densidade de probabilidade de uma variável aleatória $T$ com dois parâmetros segue uma distribuição Birnbaum-Saunders generalizada dada por

$$
\begin{array}{lll}
f_{T}(t)=\frac{1}{\sqrt{2 \pi}} \exp \left(-\frac{1}{2 \alpha^{2}}\left[\frac{t}{\beta}+\frac{\beta}{t}-2\right]\right) \frac{t^{-3 / 2}(t+\beta)}{2 \alpha \sqrt{\beta}} & \text { [Normal] } \\
f_{T}(t)=\frac{1}{\pi}\left(1+\frac{1}{\alpha^{2}}\left[\frac{t}{\beta}+\frac{\beta}{t}-2\right]\right)^{-1} \frac{t^{-3 / 2}(t+\beta)}{2 \alpha \sqrt{\beta}} & \text { [Cauchy] } \\
f_{T}(t)=\frac{1}{4} \exp \left(-\frac{1}{2 \alpha}\left[\sqrt{\frac{T}{\beta}}-\sqrt{\frac{\beta}{T}}\right]\right) \frac{t^{-3 / 2}(t+\beta)}{2 \alpha \sqrt{\beta}} & \text { [Laplace]. }
\end{array}
$$

Exemplo 2. Se $T \sim \mathcal{B S G}(\alpha, \beta ; g)$, então para uma dada função geradora de densidades $g$ espeficada em parêntesis colchetes, a função densidade de probabilidade de uma variável aleatória $T$ com três ou mais parâmetros segue uma distribuição Birnbaum-Saunders generalizada dada por

$$
\begin{aligned}
& f_{T}(t)=\frac{\Gamma([\nu+1] / 2)}{\Gamma(\nu / 2) \sqrt{\nu \pi}}\left(1+\frac{1}{\nu \alpha^{2}}\left[\frac{t}{\beta}+\frac{\beta}{t}-2\right]\right)^{-[\nu+1] / 2} \frac{t^{-3 / 2}(t+\beta)}{2 \alpha \sqrt{\beta}} \nu>0 \\
& \text { [t-Student] } \\
& f_{T}(t)=\frac{2^{-(1+1 / 2 \nu)}}{\Gamma(1+1 / 2 \nu)} \exp \left(-\frac{1}{2 \alpha^{2 \nu}}\left[\frac{t}{\beta}+\frac{\beta}{t}-2\right]^{\nu}\right) \frac{t^{-3 / 2}(t+\beta)}{2 \alpha \sqrt{\beta}} \quad \nu>0 \\
& \text { [Exponêncial potência] }
\end{aligned}
$$




$$
\begin{aligned}
f_{T}(t)= & \left.\frac{\nu r^{(2 q-1) /(2 \nu)}}{\Gamma([2 q-1] / 2 \nu)}\left(\frac{1}{\alpha^{2}}\left[\frac{t}{\beta}+\frac{\beta}{t}-2\right]\right)^{q-1} \exp \left(-\frac{r}{\alpha^{2 \nu}}\left[\frac{t}{\beta}+\frac{\beta}{t}-2\right]\right]^{\nu}\right) \frac{t^{-3 / 2}(t+\beta)}{2 \alpha \sqrt{\beta}} \\
& r, \nu>0, q>1 / 2[\text { Kotz }] .
\end{aligned}
$$

Neste trabalho estudamos as distribuições Birnbaum-Saunders e Birnbaum-Saunders-t (BS-t) dadas pelas equações (2.16) e (2.19), respectivamente. A distribuição BS-t surge como uma interessante alternativa para modelar tempos de vida na presença de observações atípicas. Existe uma relação entre as distribuições Birnbaum-Saunders generalizada e seno hiperbólico simétrica que descrevemos a seguir.

\subsection{Distribuição log-Birnbaum-Saunders generalizada}

Recordemos da equação (1.8) que se $Y \sim \mathcal{S H} \mathcal{S}(\alpha, \gamma, \sigma ; g)$, então sua função densidade de probabilidade fica dada por

$$
f_{Y}(y)=f_{Z}\left(\frac{2}{\alpha} \operatorname{senh}\left(\frac{y-\gamma}{\sigma}\right)\right) \frac{2}{\alpha \sigma} \cosh \left(\frac{y-\gamma}{\sigma}\right), \quad y \in \mathbb{R}
$$

em que $Z \sim \mathcal{S}(0,1 ; g), \alpha>0, \gamma \in \mathbb{R}$ e $\sigma>0$. Se $Y=\log (T)$, em que $T \sim \mathcal{B S G}(\alpha, \beta ; g)$, então $Y \sim \mathcal{S H} \mathcal{S}(\alpha, \gamma=\log (\beta), \sigma=2 ; g)$, conhecida como distribuição log-Birnbaum-Saunders generalizada (log-BSG) de parâmetros $\alpha$, $\gamma$ e função geradora de densidades $g$. A função densidade de probabilidade de uma variável aleatória $Y \sim \log -\operatorname{BSG}(\alpha, \gamma ; g)$ fica dada por

$$
f_{Y}(y)=\frac{1}{2} f_{Z}\left(\frac{2}{\alpha} \operatorname{senh}\left(\frac{y-\gamma}{2}\right)\right) \frac{2}{\alpha} \cosh \left(\frac{y-\gamma}{2}\right) \quad y \in \mathbb{R}
$$

\subsubsection{Distribuição log-Birnbaum-Saunders}

Rieck e Nedelman (1991) provaram que se $T \sim \mathcal{B S}(\alpha, \beta)$, então $Y=\log (T) \sim \mathcal{S H \mathcal { N }}(\alpha, \gamma, 2)$, em que $\gamma=\log (\beta)$. Por esta razão, a distribuição seno hiperbólico normal é também denominada distribuição log-Birnbaum-Saunders (log-BS) de parâmetros $\alpha$ e $\gamma$. A função densidade de probabilidade de $Y \sim \log$-BS $(\alpha, \gamma)$ é dada por

$$
f_{Y}(y)=\frac{1}{\sqrt{2 \pi \alpha^{2}}} \cosh \left(\frac{y-\gamma}{2}\right) \exp \left\{-\frac{2}{\alpha^{2}} \operatorname{senh}^{2}\left(\frac{y-\gamma}{2}\right)\right\}, \quad \alpha>0, \gamma \in \mathbb{R}, y \in \mathbb{R}
$$

\subsubsection{Distribuição log-Birnbaum-Saunders-t}

Com base na distribuição Birnbaum-Saunders-t (BS-t) dada pela equação (2.19), temos o seguinte resultado. Se $T \sim \mathcal{B S}$-t $(\alpha, \beta ; \nu)$, então $Y=\log (T) \sim \operatorname{SHt}(\alpha, \gamma=\log (\beta), \sigma=2 ; \nu)$, conhecida como distribuição log-Birnbaum-Saunders-t (log-BS-t) de parâmetros $\alpha, \gamma$ e $\nu$. A função densidade de probabilidade de uma variável aleatória $Y \sim \log$-BS-t $(\alpha, \gamma ; \nu)$ fica dada por

$$
f_{Y}(y)=\frac{\Gamma([\nu+1] / 2)}{\Gamma(\nu / 2) \sqrt{4 \nu \pi}}\left[1+\frac{4}{\nu \alpha^{2}} \operatorname{senh}^{2}\left(\frac{y-\gamma}{2}\right)\right]^{-[\nu+1] / 2} \frac{2}{\alpha} \cosh \left(\frac{y-\gamma}{2}\right) .
$$


Alguns gráficos da distribuição log-BS-t para diferentes graus de liberdade, $\alpha=1$ e $\gamma=0$ são apresentados na Figura 2.4. Baseado na Figura 2.4 (esquerda), podemos observar que a distribuição $\log$-BS-t é muito flexível para modelar a curtose e que quando os graus de liberdade $\nu$ aumentam a distribuição tende à distribuição log-BS clássica. A Figura 2.4 (direita), também estabelece que a distribuição log-BS-t possui caudas mais pesadas que as distribuições log-BS, normal e t-Student.
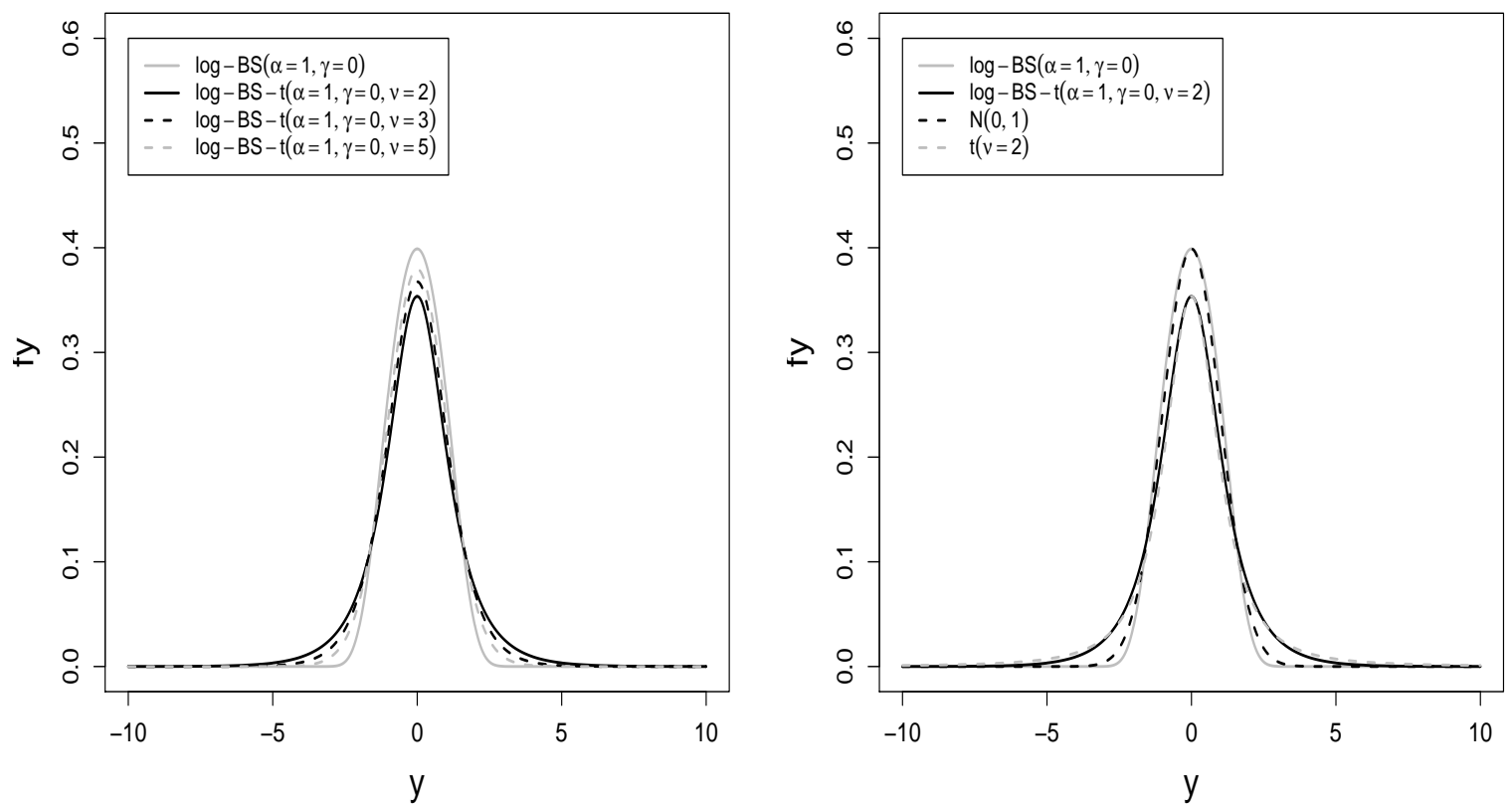

Figura 2.4: Gráfico das distribuições $\log$-BS-t $(1,0 ; \nu)$ para diferentes graus de liberdade (esquerda) e log$\mathrm{BS}(1,0), \mathcal{N}(0,1)$ e $t_{2}$ (direita).

Alguns aspectos de robustez associados ao processo de estimação por máxima verossimilhança, no modelo de regressão log-BS-t serão estudados no Capítulo 3.

\subsection{Conclusões do capítulo}

Neste capítulo foram descritas as distribuições Birnbaum-Saunders (BS) e Birnbaum-Saunders generalizada (BSG). Mostrou-se a relação que existe entre as distribuições seno hiperbólico simétrica (SHS), BSG, log-BSG, log-BS e log-BS-t. Apresentaram-se algumas propriedades e análises gráficas das distribuições BS e BSG com base nas distribuições normal, Cauchy, Laplace, t-Student, exponencial potência e Kotz. Por meio das análises gráficas, destaca-se o fato que a distribuição Birnbaum-Saunders generalizada possui diferentes graus de curtose e assimetria, além disso pode apresentar unimodalidade e bimodalidade. No próximo Capítulo serão estudados os modelos de regressão log-BS e log-BS-t de efeitos fixos. 



\section{Capítulo 3}

\section{Modelos log-BS e log-BS-t de Efeitos}

\section{Fixos}

Neste capítulo, faremos uma breve descrição a respeito dos modelos log-Birnbaum-Saunders (logBS) e log-Birnbaum-Saunders-t (log-BS-t) para o caso de modelos com efeitos fixos e supondo a existência de censura. Apresentamos a função escore, a matriz hessiana e alguns resultados assintóticos para os modelos descritos anteriormente. Além disso, são mostrados alguns aspectos de robustez relacionados ao processo de estimação por máxima verossimilhança da distribuição log-BS-t em relação à distribuição log-BS. Finalmente, descrevemos o método de influência local proposto por Cook (1986) e os resíduos tipo martingale comumente usados na área de análises de sobrevivência, veja por exemplo, Leiva et al. (2007).

\subsection{Modelos log-Birnbaum-Saunders}

Consideremos o modelo de regressão log-Birnbaum-Saunders dado por

$$
Y_{i}=\boldsymbol{x}_{i}^{\top} \boldsymbol{\beta}+\varepsilon_{i}, \quad i=1, \ldots, n,
$$

em que $Y_{i}$ é o logaritmo do tempo de falha ou o logaritmo do tempo de censura observado para o $i$-ésimo indivíduo, $\boldsymbol{\beta}=\left[\beta_{1}, \ldots, \beta_{p}\right]^{\top}$ é o vetor de parâmetros desconhecidos a serem estimados, $\boldsymbol{x}_{i}=\left[x_{i 1}, \ldots, x_{i p}\right]^{\top}$ contém valores de variáveis explicativas e $\varepsilon_{i} \sim \log$-BS $(\alpha, 0)$ é o elemento referente do erro aleatório do modelo, para $i=1, \ldots, n$. Assumimos a existência de censura não informativa e que os tempos observados de falha ou os tempos observados de censura são independentes. Sejam $O$ e $C$ os conjuntos dos indivíduos para os quais $Y_{i}$ é o logaritmo do tempo de falha ou o logaritmo do tempo de censura, respectivamente. O logaritmo da função de verossimilhança do modelo (3.1), para $\boldsymbol{\theta}=\left[\boldsymbol{\beta}^{\top} \alpha\right]^{\top}$, pode ser expresso por

$$
L(\boldsymbol{\theta}) \propto \sum_{i \in O}\left[\log \left(\xi_{i 1}\right)-\frac{\xi_{i 2}^{2}}{2}\right]+\sum_{i \in C} \log \left(1-\Phi\left(\xi_{i 2}\right)\right),
$$


em que $\Phi(\cdot)$ denota a função de distribuição acumulada de uma variável aleatória com distribuição normal padrão,

$$
\xi_{i 1}=\frac{2}{\alpha} \cosh \left(\frac{y_{i}-\boldsymbol{x}_{i}^{\top} \boldsymbol{\beta}}{2}\right)
$$

$\mathrm{e}$

$$
\xi_{i 2}=\frac{2}{\alpha} \operatorname{senh}\left(\frac{y_{i}-\boldsymbol{x}_{i}^{\top} \boldsymbol{\beta}}{2}\right), \quad \text { para } i=1, \ldots, n
$$

\subsubsection{Função escore}

A fim de obtermos as estimativas de máxima verossimilhança de $\boldsymbol{\theta}=\left[\begin{array}{ll}\boldsymbol{\beta}^{\top} \alpha\end{array}\right]^{\top}$ no modelo (3.1), devemos obter as soluções das equações

$$
U_{\beta_{j}}(\boldsymbol{\theta})=\frac{\partial L(\boldsymbol{\theta})}{\partial \beta_{j}}=0 \quad \text { e } \quad U_{\alpha}(\boldsymbol{\theta})=\frac{\partial L(\boldsymbol{\theta})}{\partial \alpha}=0, \quad \text { para } j=1, \ldots, p .
$$

Logo,

$$
U_{\beta_{j}}(\boldsymbol{\theta})=\frac{1}{2} \sum_{i \in O} x_{i j}\left[\xi_{i 1} \xi_{i 2}-\xi_{i 3}\right]+\frac{1}{2} \sum_{i \in C}\left[x_{i j} \xi_{i 1} h\left(\xi_{i 2}\right)\right]
$$

$\mathrm{e}$

$$
U_{\alpha}(\boldsymbol{\theta})=\frac{1}{\alpha} \sum_{i \in O}\left[\xi_{i 2}^{2}-1\right]+\frac{1}{\alpha} \sum_{i \in C}\left[\xi_{i 2} h\left(\xi_{i 2}\right)\right],
$$

em que

$$
h\left(\xi_{i 2}\right)=\frac{\phi\left(\xi_{i 2}\right)}{1-\Phi\left(\xi_{i 2}\right)}
$$

$\mathrm{e}$

$$
\xi_{i 3}=\tanh \left(\frac{y_{i}-\boldsymbol{x}_{i}^{\top} \boldsymbol{\beta}}{2}\right)
$$

Já que as soluções de essas equações não possuem forma fechada, é preciso o uso de métodos iterativos, para os quais podemos considerar como valores iniciais as estimativas de máxima verossimilhança do modelo log-BS sem censura; veja por exemplo, Leiva et al. (2007). Os métodos iterativos tradicionalmente usados para estimar parâmetros são os métodos de escore de Fisher, Newton-Raphson e quase-Newton. O método de quase-Newton é uma boa opção para a resolução de sistemas de equações não lineares, pois (em geral) consegue alcançar a solução em um tempo de execução menor do que os métodos mencionados anteriormente. O método de quase-Newton aproxima a matriz de segundas derivadas, reduzindo assim o número de operações por iteração; veja por exemplo, Lange (2004). 


\subsubsection{Matriz hessiana}

A seguir apresentamos a matriz hessiana do modelo (3.1), que será usada na seguinte seção como uma aproximação da matriz de variâncias e covariâncias de $\widehat{\boldsymbol{\theta}}$. A matriz hessiana do modelo (3.1) é calculada como

$$
\ddot{\boldsymbol{L}}_{\theta \theta}=\left[\begin{array}{cc}
\frac{\partial \boldsymbol{U}_{\beta}^{\top}(\boldsymbol{\theta})}{\partial \boldsymbol{\beta}} & \frac{\partial U_{\alpha}(\boldsymbol{\theta})}{\partial \boldsymbol{\beta}} \\
\frac{\partial \boldsymbol{U}_{\beta}^{\top}(\boldsymbol{\theta})}{\partial \alpha} & \frac{\partial U_{\alpha}(\boldsymbol{\theta})}{\partial \alpha}
\end{array}\right]=\left[\begin{array}{cc}
\ddot{\boldsymbol{L}}_{\beta \beta} & \ddot{\boldsymbol{L}}_{\beta \alpha} \\
\ddot{\boldsymbol{L}}_{\alpha \beta} & \ddot{L}_{\alpha \alpha}
\end{array}\right]=\left[\begin{array}{cc}
\boldsymbol{X}^{\top} \boldsymbol{V} \boldsymbol{X} & \boldsymbol{X}^{\top} \boldsymbol{k} \\
\boldsymbol{k}^{\top} \boldsymbol{X} & \operatorname{tr}(\boldsymbol{G})
\end{array}\right]
$$

em que $\boldsymbol{U}_{\beta}^{\top}(\boldsymbol{\theta})=\left[U_{\beta_{1}}(\boldsymbol{\theta}), \ldots, U_{\beta_{p}}(\boldsymbol{\theta})\right], \boldsymbol{V}=\operatorname{diag}\left\{v_{1}(\boldsymbol{\theta}), \ldots, v_{n}(\boldsymbol{\theta})\right\}, \boldsymbol{k}=\left[k_{1}(\boldsymbol{\theta}), \ldots, k_{n}(\boldsymbol{\theta})\right]^{\top} \mathrm{e}$ $\boldsymbol{G}=\operatorname{diag}\left\{g_{1}(\boldsymbol{\theta}), \ldots, g_{n}(\boldsymbol{\theta})\right\}, \operatorname{com}$

$$
\begin{aligned}
& v_{i}(\boldsymbol{\theta})= \begin{cases}\frac{1}{4} \operatorname{sech}^{2}\left(\frac{y_{i}-\boldsymbol{x}_{i}^{\top} \boldsymbol{\beta}}{2}\right)-\frac{1}{\alpha^{2}} \cosh \left(y_{i}-\boldsymbol{x}_{i}^{\top} \boldsymbol{\beta}\right), & \text { se } i \in O ; \\
-\frac{1}{4} \xi_{i 2} h\left(\xi_{i 2}\right)-\frac{1}{4} \xi_{i 1}^{2} h^{\prime}\left(\xi_{i 2}\right), & \text { se } i \in C ;\end{cases} \\
& k_{i}(\boldsymbol{\theta})= \begin{cases}-\frac{2}{\alpha^{3}} \operatorname{senh}\left(y_{i}-\boldsymbol{x}_{i}^{\top} \boldsymbol{\beta}\right), & \text { se } i \in O ; \\
-\frac{1}{2 \alpha} \xi_{i 1} h\left(\xi_{i 2}\right)-\frac{1}{\alpha^{3}} \operatorname{senh}\left(y_{i}-\boldsymbol{x}_{i}^{\top} \boldsymbol{\beta}\right) h^{\prime}\left(\xi_{i 2}\right), & \text { se } i \in C ;\end{cases}
\end{aligned}
$$

$\mathrm{e}$

$$
g_{i}(\boldsymbol{\theta})=\left\{\begin{array}{lr}
\frac{1}{\alpha^{2}}-\frac{3 \xi_{i 2}^{2}}{\alpha^{2}}, & \text { se } i \in O \\
-\frac{2}{\alpha^{2}} \xi_{i 2} h\left(\xi_{i 2}\right)-\frac{1}{\alpha^{2}} \xi_{i 2}^{2} h^{\prime}\left(\xi_{i 2}\right), & \text { se } i \in C
\end{array}\right.
$$

em que $h^{\prime}\left(\xi_{i 2}\right)$ é a derivada de $h\left(\xi_{i 2}\right)$ definida na equação (3.5), $\xi_{i 1}$ e $\xi_{i 2}$ definidas nas equações (3.3) e (3.4).

\subsubsection{Resultados assintóticos}

Com base na normalidade assintótica dos estimadores de máxima verossimilhança, tem-se que

$$
\widehat{\boldsymbol{\theta}} \dot{\sim} \mathcal{N}_{p+1}\left(\boldsymbol{\theta}, \boldsymbol{\Sigma}_{\theta}\right),
$$

$\operatorname{com} \boldsymbol{\Sigma}_{\theta}$ sendo a matriz de variâncias e covariâncias de $\widehat{\boldsymbol{\theta}}$, que pode ser aproximada pela matriz de informação observada $-\ddot{\boldsymbol{L}}_{\theta \theta}^{-1} \operatorname{com} \ddot{\boldsymbol{L}}_{\theta \theta}$ definida na equação (3.7). A seguir apresentamos os modelos log-BS-t, a função escore, a matriz hessiana e alguns resultados assintóticos associados ao modelo; veja por exemplo, Barros et al. (2008). 


\subsection{Modelos log-Birnbaum-Saunders-t}

Barros et al. (2008) estenderam o modelo log-BS de efeitos fixos para dados com censura proposto por Leiva et al. (2007), para o modelo log-BS-t de efeitos fixos para dados com censura. Consideremos o modelo de regressão log-BS-t dado por

$$
Y_{i}=\boldsymbol{x}_{i}^{\top} \boldsymbol{\beta}+\varepsilon_{i}, \quad i=1, \ldots, n,
$$

em que $Y_{i}$ denota o logaritmo do tempo observado de falha ou censura para o $i$-ésimo indivíduo $\boldsymbol{\beta}=\left[\beta_{1}, \ldots, \beta_{p}\right]^{\top}$ é um vetor de parâmetros desconhecidos, $\boldsymbol{x}_{i}=\left[x_{i 1}, \ldots, x_{i p}\right]^{\top}$ contém valores de variáveis explicativas e $\varepsilon_{i} \sim \log$-BS-t $(\alpha, 0 ; \nu)$ é o termo aleatório do modelo (3.8), para $i=1, \ldots, n$. Como no modelo log-BS, assumimos a presença de censura não informativa e independência entre os tempos de falha e os tempos de censura. Sejam $O$ e $C$ os conjuntos dos indivíduos para os quais $Y_{i}$ é o logaritmo do tempo de falha ou o logaritmo do tempo de censura, respectivamente. O logaritmo da função de verossimilhança no modelo (3.8), para $\boldsymbol{\theta}=\left[\boldsymbol{\beta}^{\top} \alpha\right]^{\top}$, pode ser expresso por

$$
L(\boldsymbol{\theta}) \propto \sum_{i \in O}\left[\log \left(\xi_{i 1}\right)-\left[\frac{\nu+1}{2}\right] \log \left(\nu+\xi_{i 2}^{2}\right)\right]+\sum_{i \in C} \log \left(1-\Phi_{t}\left(\xi_{i 2}\right)\right),
$$

em que $\Phi_{t}(\cdot)$ é a função de distribuição acumulada de uma variável aleatória t-Student padrão com $\nu$ graus de liberdade, $\xi_{i 1}$ e $\xi_{i 2}$ são definidas nas equações (3.3) e (3.4).

\subsubsection{Função escore}

A fim de obtermos as estimativas de máxima verossimilhança de $\boldsymbol{\theta}$, no modelo (3.8), devemos obter as soluções das equações

$$
U_{\beta_{j}}(\boldsymbol{\theta})=\frac{\partial L(\boldsymbol{\theta})}{\partial \beta_{j}}=0
$$

e

$$
U_{\alpha}(\boldsymbol{\theta})=\frac{\partial L(\boldsymbol{\theta})}{\partial \alpha}=0, \quad \text { para } j=1, \ldots, p .
$$

Logo,

$$
U_{\beta_{j}}(\boldsymbol{\theta})=\frac{1}{2} \sum_{i \in O} x_{i j}\left[\xi_{i 1} \xi_{i 2} w\left(\xi_{i 2}^{2}\right)-\xi_{i 3}\right]+\frac{1}{2} \sum_{i \in C}\left[x_{i j} \xi_{i 1} h_{t}\left(\xi_{i 2}\right)\right]
$$

$\mathrm{e}$

$$
U_{\alpha}(\boldsymbol{\theta})=\frac{1}{\alpha} \sum_{i \in O}\left[w\left(\xi_{i 2}^{2}\right) \xi_{i 2}^{2}-1\right]+\frac{1}{\alpha} \sum_{i \in C}\left[\xi_{i 2} h_{t}\left(\xi_{i 2}\right)\right]
$$


com

$$
w\left(\xi_{i 2}^{2}\right)=\frac{\nu+1}{\nu+\xi_{i 2}^{2}} \quad \text { e } \quad h_{t}\left(\xi_{i 2}\right)=\frac{\phi_{t}\left(\xi_{i 2}\right)}{1-\Phi_{t}\left(\xi_{i 2}\right)} .
$$

Como as equações (3.10) e (3.11) não possuem forma fechada, são necessários o uso de métodos iterativos.

Note que quando $\nu \rightarrow \infty$, tem-se que $w\left(\xi_{i 2}^{2}\right) \rightarrow 1$ para todo $i=1, \ldots, n$ e as funções escore dadas nas equações (3.10) e (3.11) se reduzem às funções escore do modelo de regressão log-BS, como é esperado, veja por exemplo, Galea et al. (2004) e Leiva et al. (2007). Assim, $w\left(\xi_{i 2}^{2}\right)$ pode ser interpretado como um peso que é inversamente proporcional à quantidade $\xi_{i 2}^{2}=\left([2 / \alpha] \operatorname{senh}\left(\left[y_{i}-\right.\right.\right.$ $\left.\left.\left.\boldsymbol{x}_{i}^{\top} \boldsymbol{\beta}\right] / 2\right)\right)^{2}$. A Figura 3.1 descreve o comportamento de $w\left(\xi_{i 2}^{2}\right)$ contra $\xi_{i 2}^{2}$ para alguns valores dos graus de liberdade $\nu$ da distribuição t-Student. Notamos que o peso decrece quando os graus de liberdade tornam-se menores, portanto temos para as estimativas de máxima verossimilhança do modelo log-BS-t um tipo de robustez contra observações atípicas no sentido da quantidade $\xi_{i 2}$, já que valores aberrantes para essa quantidade levam a pesos pequenos no processo de estimação.

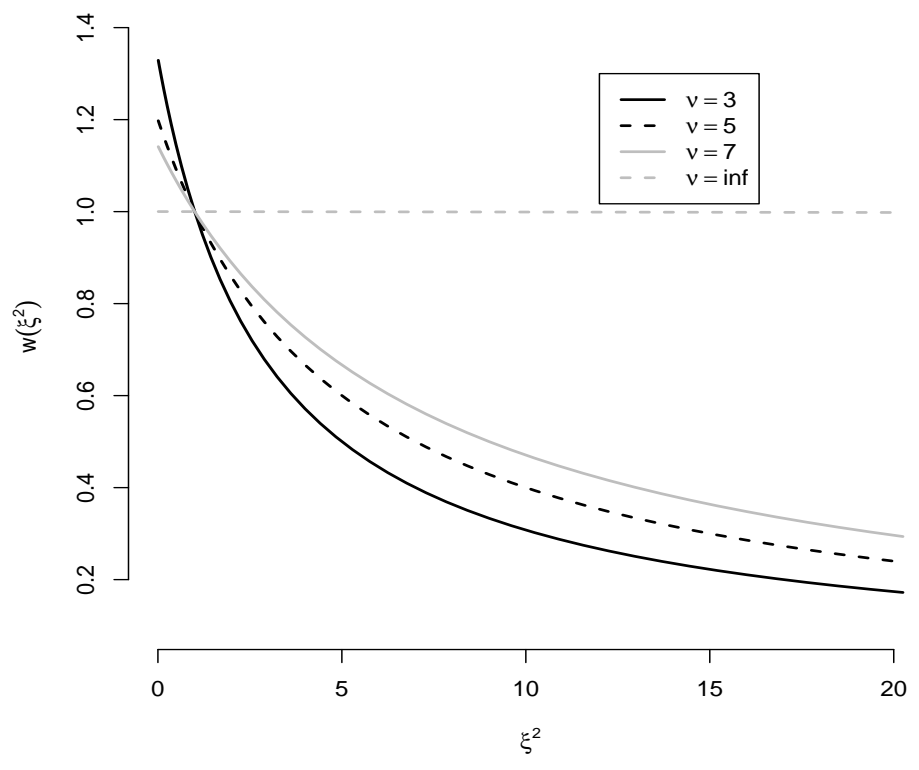

Figura 3.1: Gráfico de $w\left(\xi^{2}\right)$ versus $\xi^{2}$ para alguns valores de $\nu$ da distribuição t-Student.

\subsubsection{Matriz hessiana}

Neste caso, para o modelo (3.8), a matriz de informação observada é dada por $-\ddot{\boldsymbol{L}}_{\theta \theta}$, em que

$$
\ddot{\boldsymbol{L}}_{\theta \theta}=\left[\begin{array}{cc}
\ddot{\boldsymbol{L}}_{\beta \beta} & \ddot{\boldsymbol{L}}_{\beta \alpha} \\
\ddot{\boldsymbol{L}}_{\alpha \beta} & \ddot{L}_{\alpha \alpha}
\end{array}\right]=\left[\begin{array}{cc}
\boldsymbol{X}^{\top} \boldsymbol{V} \boldsymbol{X} & \boldsymbol{X}^{\top} \boldsymbol{k} \\
\boldsymbol{k}^{\top} \boldsymbol{X} & \operatorname{tr}(\boldsymbol{G})
\end{array}\right],
$$


em que $\boldsymbol{V}=\operatorname{diag}\left\{v_{1}(\boldsymbol{\theta}), \ldots, v_{n}(\boldsymbol{\theta})\right\}, \boldsymbol{k}=\left[k_{1}(\boldsymbol{\theta}), \ldots, k_{n}(\boldsymbol{\theta})\right]^{\top}$ e $\boldsymbol{G}=\operatorname{diag}\left\{g_{1}(\boldsymbol{\theta}), \ldots, g_{n}(\boldsymbol{\theta})\right\}, \operatorname{com}$

$$
\begin{aligned}
& v_{i}(\boldsymbol{\theta})= \begin{cases}\frac{1}{4}\left(1-\xi_{i 3}^{2}\right)-\frac{1}{\alpha^{2}} \cosh \left(y_{i}-\boldsymbol{x}_{i}^{\top} \boldsymbol{\beta}\right) w\left(\xi_{i 2}^{2}\right)-\frac{2}{\alpha^{4}} \operatorname{senh}^{2}\left(y_{i}-\boldsymbol{x}_{i}^{\top} \boldsymbol{\beta}\right) w^{\prime}\left(\xi_{i 2}^{2}\right), \text { se } i \in O ; \\
-\frac{1}{4} \xi_{i 2} h_{t}\left(\xi_{i 2}\right)-\frac{1}{4} \xi_{i 1}^{2} h_{t}^{\prime}\left(\xi_{i 2}\right), \quad \text { se } i \in C ;\end{cases} \\
& k_{i}(\boldsymbol{\theta})= \begin{cases}-\frac{2}{\alpha^{3}} \operatorname{senh}\left(y_{i}-\boldsymbol{x}_{i}^{\top} \boldsymbol{\beta}\right)\left[w\left(\xi_{i 2}^{2}\right)+\xi_{i 2}^{2} w^{\prime}\left(\xi_{i 2}^{2}\right)\right], & \text { se } i \in O ; \\
-\frac{1}{2 \alpha} \xi_{i 1} h_{t}\left(\xi_{i 2}\right)-\frac{1}{\alpha^{3}} \operatorname{senh}\left(y_{i}-\boldsymbol{x}_{i}^{\top} \boldsymbol{\beta}\right) h_{t}^{\prime}\left(\xi_{i 2}\right), & \text { se } i \in C ;\end{cases}
\end{aligned}
$$

$\mathrm{e}$

$$
g_{i}(\boldsymbol{\theta})= \begin{cases}\frac{1}{\alpha^{2}}-\frac{3 \xi_{i 2}^{2}}{\alpha^{2}} w\left(\xi_{i 2}^{2}\right)-\frac{2}{\alpha^{2}} \xi_{i 2}^{4} w^{\prime}\left(\xi_{i 2}^{2}\right), & \text { se } i \in O ; \\ -\frac{2}{\alpha^{2}} \xi_{i 2} h_{t}\left(\xi_{i 2}\right)-\frac{1}{\alpha^{2}} \xi_{i 2}^{2} h_{t}^{\prime}\left(\xi_{i 2}\right), & \text { se } i \in C ;\end{cases}
$$

em que $w^{\prime}\left(\xi_{i 2}\right)$ é a derivada de $w\left(\xi_{i 2}\right)$ definido na equação (3.11).

\subsubsection{Resultados assintóticos}

De forma análoga ao modelo log-BS e com base na normalidade assintótica dos estimadores de máxima verossimilhança, tem-se que $\widehat{\boldsymbol{\theta}} \dot{\sim} \mathcal{N}_{p+1}\left(\boldsymbol{\theta}, \boldsymbol{\Sigma}_{\theta}\right)$, com $\boldsymbol{\Sigma}_{\theta}$ sendo a matriz de variâncias e covariâncias de $\widehat{\boldsymbol{\theta}}$, a qual pode ser aproximada pela matriz de informação observada $-\ddot{\boldsymbol{L}}_{\theta \theta}^{-1}$ em que $\ddot{\boldsymbol{L}}_{\theta \theta}$ foi definido na equação (3.12).

\subsection{Análise de diagnóstico}

A eliminação de pontos é o método mais tradicional de sensibilidade que consiste em avaliar o impacto da retirada de uma observação nas estimativas dos parâmetros de um modelo. Há uma vasta literatura sobre o assunto (veja, por exemplo, Belsley et al., 1980; Chatterjee e Hadi, 1988; Cook e Weisberg, 1982; Weisberg, 2005). Contudo, o método de eliminação de pontos não verifica a influência conjunta das observações nas estimativas dos parâmetros. Nesse sentido o método de influência local tem se constituído numa ferramenta importante na análise da influência conjunta das observações nas estimativas do modelo.

\subsubsection{Influência local}

O conceito de influência local foi proposto por Cook (1986) com o objetivo de avaliar mudanças nos resultados da análise quando pequenas perturbações são incorporadas ao modelo ou dados. Se essas perturbações causarem efeitos desproporcionais, pode haver indícios de que o modelo está mal ajustado ou que podem existir afastamentos sérios das suposições feitas para o mesmo. A abordagem original baseia-se na análise do afastamento da verossimilhança $L D(\boldsymbol{\omega})=2\{L(\widehat{\boldsymbol{\theta}})-$ $\left.L\left(\widehat{\boldsymbol{\theta}}_{\boldsymbol{\omega}}\right)\right\}$, em que $\widehat{\boldsymbol{\theta}}_{\boldsymbol{\omega}}$ denota a estimativa de máxima verossimilhança sob o modelo perturbado e $\boldsymbol{\omega}=\left[\omega_{1}, \ldots, \omega_{n}\right]^{\top}$ é o vetor de perturbações aplicadas no modelo ou dados. A proposta de 
Cook (1986) é avaliar o comportamento da função $L D(\boldsymbol{\omega})$ numa vizinhança do vetor de não perturbação $\boldsymbol{\omega}_{0}$. O procedimento consiste em selecionar uma direção unitária $\boldsymbol{\ell}$, tal que $\|\boldsymbol{\ell}\|=1$, e então, considerar o gráfico de $L D\left(\boldsymbol{\omega}_{0}+a \boldsymbol{\ell}\right)$ contra $a$, com $a \in \mathbb{R}$. Esse gráfico é chamado de linha projetada. Cada linha projetada pode ser caracterizada pela curvatura normal $C_{\ell}(\widehat{\boldsymbol{\theta}}) \mathrm{em}$ torno de $a=0$.

A sugestão de Cook (1986) é considerar a direção $\ell_{\max }$ correspondente à maior curvatura $C_{\ell_{\max }}$. O gráfico de índices $\ell_{\max }$ pode mostrar os elementos que, sob pequenas perturbações, exercem notável influência sobre $L D(\boldsymbol{\omega})$. Cook (1986) mostra que a curvatura normal pode ser expressa numa forma geral como

$$
C_{\ell}(\boldsymbol{\theta})=2\left|\ell^{\top} \boldsymbol{\Delta}^{\top} \ddot{\boldsymbol{L}}_{\widehat{\theta} \hat{\theta}}^{-1} \boldsymbol{\Delta} \boldsymbol{\ell}\right|,
$$

em que $\Delta$ é uma matriz $(p+1) \times n$ com

$$
\Delta_{i j}=\partial^{2} L(\boldsymbol{\theta} \mid \boldsymbol{\omega}) / \partial \theta_{i} \partial \omega_{j}, \quad i=1, \ldots, p+1 \quad \text { e } j=1, \ldots, n,
$$

com todas as quantidades sendo avaliadas em $\boldsymbol{\omega}=\boldsymbol{\omega}_{0}$ e $\boldsymbol{\theta}=\widehat{\boldsymbol{\theta}}$. Então, $C_{\ell_{\max }}$ é o maior autovalor da matriz

$$
\boldsymbol{B}=\boldsymbol{\Delta}^{\top} \ddot{\boldsymbol{L}}_{\widehat{\theta} \widehat{\theta}}^{-1} \boldsymbol{\Delta}
$$

e $\ell_{\max }$ é o correspondente autovetor (veja, por exemplo, Galea et al., 2004). Nesse contexto, se o interesse é avaliar a influência parcial em um subconjunto de $\boldsymbol{\theta}=\left(\boldsymbol{\theta}_{1}^{\top} \boldsymbol{\theta}_{2}^{\top}\right)^{\top}, \boldsymbol{\theta}_{1}$ por exemplo, tem-se que a curvatura normal na direção do vetor $\ell$ é dada por

$$
C_{\ell}\left(\boldsymbol{\theta}_{1}\right)=2\left|\ell^{\top} \boldsymbol{\Delta}^{\top}\left(\ddot{\boldsymbol{L}}_{\widehat{\theta} \widehat{\theta}}^{-1}-\boldsymbol{B}_{1}\right) \boldsymbol{\Delta} \boldsymbol{\ell}\right|,
$$

com

$$
\boldsymbol{B}_{1}=\left[\begin{array}{cc}
\mathbf{0} & \mathbf{0} \\
\mathbf{0} & \ddot{\boldsymbol{L}}_{\widehat{\theta}_{2} \widehat{\theta}_{2}}^{-1}
\end{array}\right],
$$

e $\ddot{\boldsymbol{L}}_{\widehat{\theta}_{2} \widehat{\theta}_{2}}=\left.\left\{\partial^{2} L(\boldsymbol{\theta} \mid \boldsymbol{\omega}) / \partial \boldsymbol{\theta}_{2}^{\top} \partial \boldsymbol{\theta}_{2}\right\}\right|_{\boldsymbol{\theta}=\widehat{\boldsymbol{\theta}}}$.

O gráfico do autovetor associado ao maior autovalor da matriz $\boldsymbol{\Delta}^{\top}\left(\ddot{\boldsymbol{L}}_{\widehat{\theta} \hat{\theta}}^{-1}-\boldsymbol{B}_{1}\right) \boldsymbol{\Delta}$ contra o índice das observações pode revelar quais observações estão influenciando $\boldsymbol{\theta}_{1}$. Analogamente, se o interesse está em $\boldsymbol{\theta}_{2}$, então a curvatura normal na direção do vetor $\ell$ é dada por

$$
C_{\ell}\left(\boldsymbol{\theta}_{2}\right)=2\left|\ell^{\top} \boldsymbol{\Delta}^{\top}\left(\ddot{\boldsymbol{L}}_{\widetilde{\theta} \widehat{\theta}}^{-1}-\boldsymbol{B}_{2}\right) \boldsymbol{\Delta} \boldsymbol{\ell}\right|
$$


com

$$
\boldsymbol{B}_{2}=\left[\begin{array}{cc}
\ddot{\boldsymbol{L}}_{\widehat{\theta}_{1} \widehat{\theta}_{1}}^{-1} & \mathbf{0} \\
\mathbf{0} & \mathbf{0}
\end{array}\right],
$$

e $\ddot{\boldsymbol{L}}_{\widehat{\theta}_{1} \widehat{\theta}_{1}}=\left.\left\{\partial^{2} L(\boldsymbol{\theta} \mid \boldsymbol{\omega}) / \partial \boldsymbol{\theta}_{1}^{\top} \partial \boldsymbol{\theta}_{1}\right\}\right|_{\boldsymbol{\theta}=\widehat{\boldsymbol{\theta}}}$. A influência local das observações em $\widehat{\boldsymbol{\theta}}_{2}$ pode ser avaliada considerando o gráfico $\ell_{\max }$ para a matriz $\boldsymbol{\Delta}^{\top}\left(\ddot{\boldsymbol{L}}_{\widehat{\theta} \widehat{\theta}}^{-1}-\boldsymbol{B}_{2}\right) \boldsymbol{\Delta}$ contra o índice das observações.

\subsubsection{Influência local total}

Além de considerar a direção do vetor de máxima curvatura normal $\ell_{\max }$, é possível considerar a direção $\ell=\boldsymbol{e}_{\text {in }}$ (veja, por exemplo, Lesaffre e Verbeke, 1998) a qual corresponde à direção da $i$-ésima observação, em que $\boldsymbol{e}_{i n}$ é um vetor $n \times 1$ de zeros com um 1 na $i$-ésima posição. Nesse caso, a influência local total do $i$-ésimo caso é dada por

$$
C_{i}=2\left|b_{i i}\right|, \quad i=1, \ldots, n,
$$

em que $b_{i i}$ é o $i$-ésimo elemento diagonal de $\boldsymbol{B}$. Verbeke e Molenberghs (2000) propõem considerar os casos tais que $C_{i}>2 \bar{C}$, em que

$$
\bar{C}=\frac{1}{n} \sum_{i=1}^{n} C_{i},
$$

como potenciais pontos influentes.

\subsubsection{Influência local conformal}

Embora o método baseado na curvatura normal proposto por Cook (1986) seja muito útil para avaliar potenciais pontos influentes, o mesmo apresenta algumas desvantagens. Poon e Poon (1999) observaram que a curvatura normal pode assumir qualquer valor e não é invariante sob uma transformação uniforme de escala. A fim de resolver esse problema, Poon e Poon (1999) definem uma nova medida de curvatura que tem uma interpretação mais simples com boas propriedades, que denominaram como curvatura normal conformal. Essa curvatura na direção de um vetor $\ell$ avaliada no ponto $\omega_{0}$ é dada por

$$
B_{\boldsymbol{\ell}}=\frac{\left|\boldsymbol{\ell}^{\top} \boldsymbol{\Delta}^{\top} \ddot{\boldsymbol{L}}_{\widehat{\theta} \widehat{\theta}}^{-1} \boldsymbol{\Delta} \boldsymbol{\ell}\right|}{\left\|\boldsymbol{\Delta}^{\top} \ddot{\boldsymbol{L}}_{\widehat{\theta} \widehat{\theta}}^{-1} \boldsymbol{\Delta}\right\|_{\mathcal{F}}},
$$

em que $\|\cdot\|_{\mathcal{F}}$ denota a norma de Fröbenius definida por

$$
\|\mathbf{A}\|_{\mathcal{F}}=\sqrt{\operatorname{tr}\left(\mathbf{A}^{\top} \mathbf{A}\right)}
$$

sendo A uma matriz $m \times n$. É possível provar que para qualquer direção $\ell, B_{\ell}$ satisfaz a condição $0 \leq\left|B_{\ell}\right| \leq 1$. Isso permite comparar a curvatura normal em diferentes modelos, ou seja, para 
diferentes famílias de modelos, ver por exemplo, Osorio et al. (2007). A curvatura normal conformal $B_{E_{i}}$ da perturbação básica do vetor $E_{i}$, isto é o vetor com o valor 1 na $i$-ésima posição e zeros nas demais posições, é dada por

$$
B_{E_{i}}=\sum_{j=1}^{n} \varphi_{j} a_{j i}^{2}, \quad i=1,2, \ldots
$$

em que $\varphi_{j}$ denota o valor absoluto do $j$-ésimo autovalor normalizado da matriz $\boldsymbol{B}$ e $a_{j i}$ denota o $i$-ésimo elemento do autovetor normalizado associado com $\varphi_{j}$. Poon e Poon (1999) sugerem o uso de $2 b$ como possíveis pontos de corte $B_{E_{i}}$, em que

$$
b=\frac{\operatorname{tr}(\boldsymbol{B})}{\sqrt{\operatorname{tr}\left(\boldsymbol{B}^{\top} \boldsymbol{B}\right)}} .
$$

Então, os casos tais que $B_{E_{i}}>2 b$ são considerados como potenciais pontos influentes. A seguir discutiremos brevemente a análise de resíduos para os modelos log-BS e log-BS-t.

\subsubsection{Análise de resíduos}

Therneau et al. (1990) propuseram o resíduo componente do desvio em processos de contagem usando basicamente resíduos martingales. Os resíduos martingales são assimétricos, assumem valor máximo em +1 e valor mínimo em $-\infty$.

$\mathrm{Na}$ área paramétrica, os resíduos martingales podem ser expressos como $r_{\mathrm{M}_{i}}=\delta_{i}+$ $\log \left(S_{Y}\left(y_{i} ; \widehat{\boldsymbol{\theta}}\right)\right)$, com $\delta_{i}=0,1$, indicando se a observação é censurada ou não (veja, por exemplo Klein e Moeschberger, 1997).

O resíduo componente do desvio proposto por Therneau et al. (1990) é uma transformação do resíduo martingale com a finalidade de atenuar a assimetria. Essa transformação foi motivada pelos resíduos componente do desvio encontrados em modelos lineares generalizados. Em particular, o resíduo componente do desvio para o modelo de Cox com variáveis explicativas não dependendo do tempo é expresso como

$$
r_{\mathrm{MT}_{i}}=\operatorname{sign}\left(r_{\mathrm{M}_{i}}\right) \sqrt{-2\left[r_{\mathrm{M}_{i}}+\delta_{i} \log \left(\delta_{i}-r_{\mathrm{M}_{i}}\right)\right]},
$$

em que $\delta_{i}=0,1$, indicando se a observação é censurada ou não, respectivamente, enquanto

$$
r_{\mathrm{M}_{i}}=\delta_{i}+\log \left(\Phi\left(-\frac{2}{\widehat{\alpha}} \operatorname{senh}\left[\frac{y_{i}-\boldsymbol{x}_{i}^{\top} \widehat{\boldsymbol{\beta}}}{2}\right]\right)\right)
$$

é o resíduo martingale para o modelo log-BS e

$$
r_{\mathrm{M}_{i}}=\delta_{i}+\log \left(\Phi_{t}\left(-\frac{2}{\widehat{\alpha}} \operatorname{senh}\left[\frac{y_{i}-\boldsymbol{x}_{i}^{\top} \widehat{\boldsymbol{\beta}}}{2}\right]\right)\right)
$$


é o resíduo martingale para o modelo log-BS-t com $\Phi(\cdot)$ e $\Phi_{t}(\cdot)$ denotando, respectivamente, as funções de distribuição acumulada da distribuição normal padrão e da distribuição t-Student padrão $\operatorname{com} \nu$ graus de liberdade.

Usaremos os resíduos $r_{\mathrm{MT}_{i}}$ como uma transformação dos resíduos martingales com o fim de obtermos resíduos distribuídos simetricamente em torno do zero. Chamaremos este resíduo de resíduo tipo martingale.

A fim de detectar afastamentos sérios das suposições feitas para os erros dos modelos log-BS e $\log$-BS-t um resíduo tipo martingale tem sido recomendado (veja, por exemplo, Leiva et al., 2007; Barros et al., 2008). O resíduo tipo martingale tem sido aplicado recentemente em alguns modelos paramétricos para a análise de dados de sobrevivência. Por exemplo, Ortega et al. (2008) desenvolveram um estudo de simulação com alguns tipos de resíduos sugeridos para a análise de modelos log-gama generalizados com censura aleatória e verificaram uma boa concordância entre a distribuição empírica do resíduo tipo martingale com a distribuição normal padrão. Isso sugere que as análises de resíduos tradicionais recomendados para os normais lineares como descritos, por exemplo, em Montgomery et al. (2001) possam ser estendidos para os modelos log-gama generalizados com censura aleatória. Leiva et al. (2007), Barros et al. (2008) e Ortega et al. (2009) desenvolveram estudos de simulação com o objetivo de conhecer a distribuição empírica do resíduo tipo martingale $r_{\mathrm{MT}_{i}}$ em modelos log-BS, log-BS-t e log-gama generalizados com fração de cura, respectivamente. Os resultados obtidos foram muito similares àqueles obtidos por Ortega et al. (2008). Assim, há indicações de que o resíduo tipo martingale seja uma opção interessante nas análises de diagnóstico em alguns modelos paramétricos com censura aleatória para a análise de dados de sobrevivência.

\subsection{Conclusões do capítulo}

Neste capítulo, foram descritos os modelos de regressão log-BS (veja, por exemplo, Leiva et al., 2007) e log-BS-t (veja, por exemplo, Barros et al., 2008) de efeitos fixos para dados que apresentam censura. Além disso, foram apresentadas a função escore, a matriz hessiana e alguns resultados assintóticos que podem ser consultados com mais detalhes em Barros (2007). Foi estudada a metodologia de influência local proposta por Cook (1986), a curvatura normal conformal (veja, por exemplo, Poon e Poon, 1999), junto com os resíduos tipo martingale (veja por exemplo, Leiva et al., 2007; Barros et al., 2008). 


\section{Capítulo 4}

\section{Modelos log-Birnbaum-Saunders mistos}

O principal objetivo deste capítulo é introduzir os modelos log-Birnbaum-Saunders (log-BS) para dados correlacionados (ou modelos de efeitos aleatórios), isto é, estender os modelos log-BS de efeitos fixos apresentados na Seção 3.1. Os modelos de efeitos aleatórios surgem como uma boa alternativa para ajustar modelos multiníveis, dados longitudianis e dados de medidas repetidas. Para motivar este capítulo, consideremos o conjunto de dados estudado por Smith (1991). Esses dados correspondem aos tempos de falha (horas) de um tipo particular de fibra (Kevlar 49) submetido a diferentes níveis de estresse medidos em MPa (Mega Pascal). Uma amostra de oito carretéis foi selecionada para o estudo desde uma população de carretéis e portanto o efeito do carretel pode ser considerado como um efeito aleatório. Este exemplo será analizado com mais detalhes no Capítulo 6.

Apresentamos, inicialmente, uma revisão dos métodos de quadratura de Gauss-Hermite não adaptativa e adaptativa, unidimensional e multidimensional, respectivamente. As principais referências para o caso unidimensional são os artigos de Liu e Pierce (1994) e Lesaffre e Spiessens (2001); e para o caso multidimensional são os artigos de Pinheiro e Bates (1995) e Pinheiro e Chao (2006). O procedimento Proc NLMIXED do SAS foi utilizado para a obtenção das estimativas de máxima verossimilhança e dos respectivos erros padrão aproximados do modelo marginal. Em seguida, aplicamos o método de quadratura de Gauss-Hermite não adaptativo em modelos log-BS com intercepto aleatório. Derivamos o logaritmo aproximado da função de verossimilhança marginal e daí derivamos a função escore e a matriz hessiana. Discutimos a estimação de parâmetros, predição dos efeitos aleatórios e teste para o componente de variância. A partir do logaritmo aproximado da função de verossimilhança derivamos as curvaturas normais de influência local para alguns esquemas usuais de perturbação. Finalmente, discutimos a extensão do resíduo tipo martingale para os modelos log-BS mistos. 


\subsection{Quadratura de Gauss-Hermite}

\subsubsection{Versão unidimensional}

Nesta seção apresentamos os métodos de quadratura de Gauss-Hermite unidimensional, não adaptativa e adaptativa.

\section{(i) Versão não adaptativa}

As técnicas de integração numérica representam uma forma de se obterem aproximações para integrais que não podem ser resolvidas analiticamente. A regra de Simpson e os métodos de quadratura são métodos interessantes dentre as técnicas para integrais univariadas (veja, por exemplo, Davis e Rabinowitz, 1975). Esses métodos consistem em reexpressar uma função $g(x)$ : $\mathbb{R} \rightarrow \mathbb{R}$ como o produto de uma função de pesos $v(x): \mathbb{R} \rightarrow \mathbb{R}^{+}$e outra função $f(x): \mathbb{R} \rightarrow \mathbb{R}$, isto é

$$
g(x)=f(x) v(x)
$$

Definição 6. (quadratura de Gauss-Hermite não adaptativa unidimensional)

Se $g(x)$ é uma função suave a quadratura de Gauss-Hermite não adaptativa, ou simplesmente quadratura de Gauss-Hermite é definida como

$$
\int_{-\infty}^{+\infty} g(x) \mathrm{d} x=\int_{-\infty}^{+\infty} f(x) \exp \left\{-x^{2}\right\} \mathrm{d} x \approx \sum_{k=1}^{q} v_{k} f\left(s_{k}\right)
$$

em que $s_{k}$ é o k-ésimo zero do polinômio de Hermite de grau q, isto é $H_{q}(x)$, e o peso $v_{k}$ depende do número $q$ de pontos da quadratura e do polinômio de Hermite $H_{q-1}(x)$ avaliado em $s_{k}$.

Tabelas para se obter os zeros e os pesos das expressões da quadratura podem ser encontradas em Abramowitz e Stegun (1972). Quando $g(x)$ é um polinômio de grau $2 q-1$, a soma (4.1) produz o valor exato da integral. Isso significa que a aproximação da integral na equação (4.1) chega a ser exata quando o número $q$ de pontos de quadratura é aumentado.

Para a maioria dos problemas de integração, a função densidade de probabilidade de uma variável aleatória $X \sim \mathcal{N}\left(\mu, \sigma^{2}\right)$ pode ser deduzida como uma função de peso. Tal função de peso é a base da quadratura de Gauss-Hermite. Para tanto, consideramos mais geralmente a função

$$
g(x)=f(x) v(x), \quad \text { com } \quad v(x)=\frac{1}{\sqrt{2 \pi \sigma^{2}}} \exp \left\{-\frac{(x-\mu)^{2}}{2 \sigma^{2}}\right\} .
$$

Se definimos $z=(x-\mu) / \sqrt{2 \sigma^{2}}$, então com base na equação (4.2) temos a identidade

$$
\int_{-\infty}^{+\infty} g(x) \mathrm{d} x=\int_{-\infty}^{+\infty} f(x) v(x) \mathrm{d} x=\int_{-\infty}^{+\infty} \pi^{-1 / 2} f(\sqrt{2} \sigma z+\mu) \exp \left\{-z^{2}\right\} \mathrm{d} z
$$


que pode ser resolvida pela quadratura de Gauss-Hermite do seguinte modo:

$$
\int_{-\infty}^{+\infty} g(x) \mathrm{d} x=\int_{-\infty}^{+\infty} f(x) v(x) \mathrm{d} x \approx \sum_{k=1}^{q} \frac{v_{k}}{\sqrt{\pi}} f\left(\sqrt{2} \sigma s_{k}+\mu\right),
$$

em que $v_{k}$ e $s_{k}$ são o $k$-ésimo peso e o $k$-ésimo zero tabulado do polinômio de Hermite de grau $q$, respectivamente.

Observação 4. Há um pacote desenvolvido no $R$ para calcular os zeros e os pesos do polinômio de Hermite. O pacote é glmmML e a função ghq, cujo código fonte foi desenvolvido em Fortran. Um exemplo da função ghq é apresentada a seguir com $q=10$ pontos de quadratura.

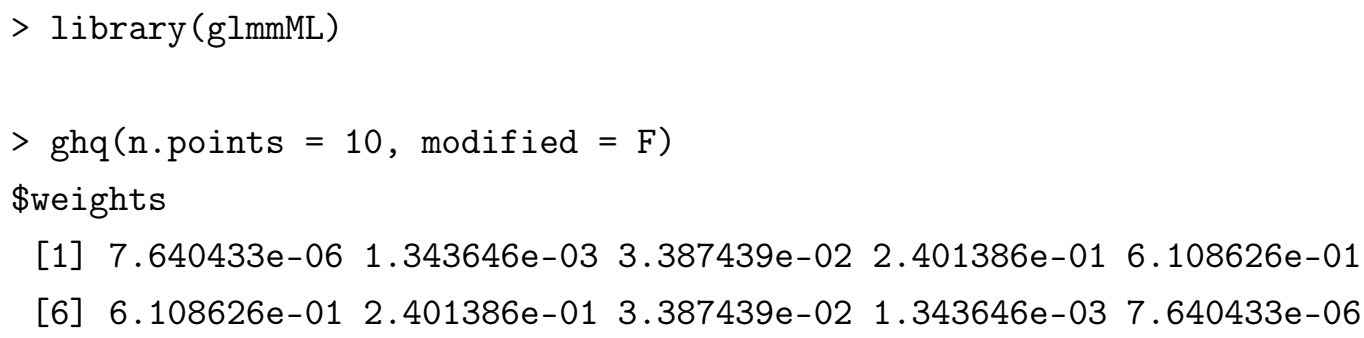

Os valores acima, podem ser comprovados em Abramowitz e Stegun (1972), página 924. Nas tabelas de Abramowitz e Stegun (1972) somente são calculados os pesos e os zeros para $q=$ $2,3,4,5,6,7,8,9,10,12,16$ e 20 pontos de quadratura do polinômio de Gauss-Hermite.

Segundo Davis e Rabinowitz (1975), uma regra de integração deveria ter pelo menos as seguintes propriedades para reduzir o esforço numérico e o erro de integração em (4.1):

$\star$ Os zeros e pesos devem ser facilmente encontrados e calculados.

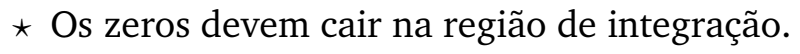

$\star$ Os pesos devem ser todos positivos.

\subsubsection{Fórmulas de Gauss-Hermite}

Algumas fórmulas úteis para o método de quadratura de Gauss-Hermite, em que $H_{q}(x)$ representa o polinômio de Gauss-Hermite de grau $q$ e $v_{k}$ o $k$-ésimo peso do polinômio de Gauss-Hermite de grau $q$, são apresentadas a seguir e posteriormente apresentamos uma tabela com alguns valores de $H_{q}(x)$ e $v_{k}$ para $q=2,3$ e 4 .

$$
\begin{aligned}
H_{q}(x) & =(-1)^{q} \exp \left\{x^{2}\right\} \frac{\mathrm{d}^{q}}{\mathrm{~d} x^{q}}\left(\exp \left\{-x^{2}\right\}\right) \mathrm{e} \\
v_{k} & =\frac{2^{q+1} q ! \sqrt{\pi}}{\left[H_{q}^{\prime}(x)\right]^{2}} \quad \text { (função que não depende de } k \text { ). }
\end{aligned}
$$


Tabela 4.1: Valores de $H_{q}(x)$ e $v_{k}$ para $q=2,3$ e 4

\begin{tabular}{cccc}
\hline$q$ & 2 & 3 & 4 \\
\hline$H_{q}(x)$ & $4 x^{2}-1$ & $8 x^{3}-12 x$ & $16 x^{4}-48 x^{2}+12$ \\
$v_{k}$ & $\frac{\sqrt{\pi}}{4 x^{2}}$ & $\frac{96 \sqrt{\pi}}{\left(24 x^{2}-12\right)^{2}}$ & $\frac{768 \sqrt{\pi}}{\left(64 x^{3}-96 x\right)^{2}}$ \\
\hline
\end{tabular}

Exemplo 3. Se aplicamos a quadratura de Gauss-Hermite com dois pontos de quadratura, na integral

$$
\int_{-\infty}^{+\infty} \exp \left\{-x^{2}\right\} x^{2} \mathrm{~d} x
$$

temos que

$$
\int_{-\infty}^{+\infty} \exp \left\{-x^{2}\right\} f(x) \mathrm{d} x \approx \frac{\sqrt{\pi}}{2}\left[f\left(\frac{\sqrt{2}}{2}\right)+f\left(-\frac{\sqrt{2}}{2}\right)\right],
$$

em que $f(x)=x^{2}$ e $H_{2}(x)=(-1)^{2} \exp \left\{x^{2}\right\} \frac{\mathrm{d}^{2}}{\mathrm{~d} x^{2}}\left(\exp \left\{-x^{2}\right\}\right)=4 x^{2}-1$. Os zeros deste polinômio são $x_{1}=+\sqrt{2} / 2$ e $x_{2}=-\sqrt{2} / 2$. Logo, $v_{1}=v_{2}=\frac{\sqrt{\pi}}{4(\sqrt{2} / 2)^{2}}=\frac{\sqrt{\pi}}{2}$. Assim, obtemos

$$
\int_{-\infty}^{+\infty} \exp \left\{-x^{2}\right\} f(x) \mathrm{d} x=\frac{\sqrt{\pi}}{2}
$$

que é o valor exato da integral.

Uma pergunta que não foi respondida até agora está relacionada ao número de pontos de quadratura do polinômio de Gauss-Hermite que devemos considerar para obter uma boa aproximação. A seguir discutimos um critério para determinar o número de pontos que são necessesários para atingir uma boa aproximação e apresentamos uma fórmula associada ao erro de arrendodamento sob o contexto dos polinômios mencionados anteriormente.

\subsubsection{Erro nas fórmulas de Gauss-Hermite}

Alguns comentários prévios à definição do erro nas fórmulas de Gauss-hermite são os seguintes:

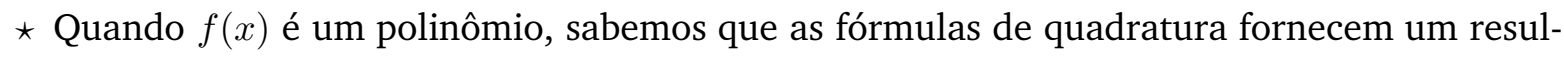
tado exato a menos, é claro, dos erros de arredondamento.

* Na maioria das situações reais, $f(x)$ não é um polinômio e, portanto, sua integral é aproximada quando calculada através das fórmulas de quadratura.

* Exibiremos uma expressão do termo do resto para a QGH. 
Não nos preocuparemos com a dedução de tais expressões por ser extremamente trabalhosas e sem nenhum interesse prático. A expressão do erro, para a fórmula de Gauss-Hermite, é dada por (veja, por ejemplo, Cuminato, 2009)

$$
E_{q}=\frac{q ! \sqrt{\pi}}{2^{q}(2 q) !} f^{(2 q)}(\xi)
$$

Como pode ser observado, a fórmula do erro na equação (4.4) contém a derivada da $f$ de ordem $2 q$, em que $q$ é o índice do último ponto considerado no cálculo da integral. Assim, usar a fórmula do erro para obter o número de pontos necessários para calcular a integral com uma determinada precisão torna-se inviável.

\subsubsection{Quantos pontos de quadratura são necessários para uma boa aproximação?}

Se quisermos o resultado da integral com uma determinada precisão, começamos calculando a integral com 2 pontos, vamos aumentando o número de pontos e comparando os resultados obtidos. Quando 2 resultados consecutivos tiverem o mesmo número de casas decimais iguais teremos o resultado com a precisão desejada. A seguir um exemplo relacionado com a distribuição normal.

Exemplo 4. Se $X \sim \mathcal{N}\left(\mu, \sigma^{2}\right)$, então $E(\exp \{X\})=\exp \left\{\mu+\sigma^{2} / 2\right\}$. Se $\mu=4$ e $\sigma^{2}=4$, então $E(\exp \{X\})=403,4288$. A seguir aproximamos $E(\exp \{X\})$ para $q=5,8,9,10$ e 11 pontos de quadratura.

1. $\operatorname{Com} q=5$ pontos de quadratura, $E(\exp \{X\}) \approx 398,6570$.

2. Com $q=8$ pontos de quadratura, $E(\exp \{X\}) \approx 403,4109$.

3. $\operatorname{Com} q=9$ pontos de quadratura, $E(\exp \{X\}) \approx 403,4267$.

4. Com $q=10$ pontos de quadratura, $E(\exp \{X\}) \approx 403,4286$.

5. Com $q=11$ pontos de quadratura, $E(\exp \{X\}) \approx 403,4288$.

Assim, com $q=11$ pontos de quadratura, temos obtido o resultado da integral com três casas decimais corretas.

\section{(ii) Versão adaptativa}

A quadratura de Gauss-Hermite, é útil na aproximação de integrais do tipo

$$
\int_{-\infty}^{+\infty} g(x) \mathrm{d} x=\int_{-\infty}^{+\infty} f(x) v(x) \mathrm{d} x, \text { em que } v(x)=v\left(x ; \mu, \sigma^{2}\right)=\frac{1}{\sqrt{2 \pi \sigma^{2}}} \exp \left\{-\frac{1}{2} \frac{(x-\mu)^{2}}{\sigma^{2}}\right\} .
$$

Porém, os pontos da quadratura $x_{k}$ são escolhidos com base em $v(x)$, independente da função $g(x)$ no integrando. Dependendo da forma de $g(x)$, os pontos $x_{k}$ selecionados podem estar ou não na região de interesse da função que se deseja aproximar. Por isso, esse método funciona de forma satisfatória nos casos em que o comportamento de $g(x)=f(x) v(x)$ for próximo de 
$v(x)$, isto é, como uma função densidade de probabilidade normal padrão. Neste caso, a função $g(x)$, deverá ser, por exemplo, um polinômio de baixa ordem. Uma boa referência que pode ser consultada para esse propósito é o trabalho de Liu e Pierce (1994). Usando o método adaptado de Gauss-Hermite, os pontos da quadratura são reescalados e modificados de forma que mais pontos da quadratura estejam na região de interesse. Lesaffre e Spiessens (2001) estudaram a quadratura de Gauss-Hermite adaptativa no modelo logístico de intercepto aleatório, mostrando que o número de pontos de quadratura depende do tipo de modelo que se esteja estudando e que a versão adaptativa é mais eficiente computacionalmente que a versão não adaptativa. Baseados na equação (4.3), escolheremos $\mu$ e $\sigma^{2}$ de modo que $g(x)$ será amostrado em uma região apropriada. Em particular, considerando $\hat{\mu}$ e $\hat{\sigma}^{2}$ como

$$
\hat{\mu}=\arg \max _{x} g(x) \text { e } \hat{\sigma}^{2}=\left.\left[-\frac{\mathrm{d}^{2}}{\mathrm{~d} x^{2}} \log g(x)\right]^{-1}\right|_{x=\hat{\mu}},
$$

em que $\hat{\mu}$ e $\hat{\sigma}^{2}$ são a média e a variância da distribuição. Se definirmos

$$
h(x)=\frac{g(x)}{v\left(x ; \hat{\mu}, \hat{\sigma}^{2}\right)},
$$

então podemos reescrever

$$
\int_{-\infty}^{+\infty} g(x) \mathrm{d} x=\int_{-\infty}^{+\infty} h(x) v\left(x ; \hat{\mu}, \hat{\sigma}^{2}\right) \mathrm{d} x
$$

em que $v\left(x ; \hat{\mu}, \hat{\sigma}^{2}\right)=\frac{1}{\sqrt{2 \pi \hat{\sigma}^{2}}} \exp \left\{-\frac{1}{2} \frac{(x-\hat{\mu})^{2}}{\hat{\sigma}^{2}}\right\}$. Se aplicarmos a quadratura de Gauss-Hermite na equação (4.5), temos que

$$
\int_{-\infty}^{+\infty} g(x) \mathrm{d} x \approx \sum_{k=1}^{q} \frac{v_{k}}{\sqrt{\pi}} h\left(\sqrt{2} \hat{\sigma} s_{k}+\hat{\mu}\right)=\sqrt{2 \pi} \hat{\sigma} \sum_{k=1}^{q} v_{k}^{+} g\left(s_{k}^{+}\right),
$$

em que $v_{k}^{+}=v_{k} \exp \left\{s_{k}^{2}\right\}$ e $s_{k}^{+}=\sqrt{2} \hat{\sigma} s_{k}+\hat{\mu}$. As quantidades $v_{k}$ e $s_{k}$ são definidas na equação (4.3).

Observação 5. Na equação (4.6), quando $q=1$, temos a aproximação de Laplace (veja, por exemplo, McCulloch e Searle, 2001), isto é

$$
\int_{-\infty}^{+\infty} g(x) \mathrm{d} x \approx h(\hat{\mu})=\sqrt{2 \pi} \hat{\sigma} g(\hat{\mu}) .
$$

A aproximação de Laplace é frequentemente usada em inferência Bayesiana para estimar densidades a posteriori e distribuições preditivas. Os principais trabalhos nessa área são de Tierney e Kadane (1986) e Leonard et al. (1989). 


\subsubsection{Versão multidimensional}

Nesta seção apresentamos os métodos de quadratura de Gauss-Hermite multidimensional, não adaptativa e adaptativa.

\section{(i) Versão não adaptativa}

Consideremos o caso $q$-dimensional em que $\boldsymbol{x}=\left[x_{1}, \ldots, x_{q}\right]^{\top}$, com uma função regular $f(\boldsymbol{x})$ : $\mathbb{R}^{q} \rightarrow \mathbb{R}$, uma função de pesos $v(\boldsymbol{x}): \mathbb{R}^{q} \rightarrow \mathbb{R}^{+}$e outra função $g(\boldsymbol{x}): \mathbb{R}^{q} \rightarrow \mathbb{R}$, definida como $g(\boldsymbol{x})=f(\boldsymbol{x}) v(\boldsymbol{x})$.

Definição 7. (quadratura de Gauss-Hermite q-dimensional não adaptativa)

Se $g(\boldsymbol{x})$ é uma função regular a quadratura de Gauss-Hermite q-dimensional não adaptativa, ou simplesmente quadratura de Gauss-Hermite, é definida como (veja, por exemplo, Fahrmeir e Tutz, 1994)

$$
\int_{\mathbb{R}^{q}} g(\boldsymbol{x}) \mathrm{d} \boldsymbol{x}=\int_{\mathbb{R}^{q}} f(\boldsymbol{x}) \exp \left\{-\boldsymbol{x}^{\top} \boldsymbol{x}\right\} \mathrm{d} \boldsymbol{x} \approx \sum_{i_{1}=1}^{N_{G H}} \ldots \sum_{i_{q}=1}^{N_{G H}} f\left(s_{i_{1}}, \ldots, s_{i_{q}}\right) \prod_{k=1}^{q} v_{i_{k}},
$$

em que $s_{i_{r}}$ é o $i_{r}$-ésimo zero do polinômio de Hermite de grau $N_{G H}$, isto é $H_{N_{G H}}(x)$, e $v_{i_{r}}$ é o peso correspondente.

A seguir definimos uma versão mais eficiente que a quadratura de Gauss-Hermite não adaptativa (veja, por exemplo, Pinheiro e Bates, 1995).

\section{(ii) Versão adaptativa}

Definição 8. (quadratura de Gauss-Hermite q-dimensional adaptativa)

Se $g(\boldsymbol{x})$ é uma função regular a quadratura de Gauss-Hermite q-dimensional adaptativa é definida como

$$
\begin{aligned}
\int_{\mathbb{R}^{q}} g(\boldsymbol{x}) \mathrm{d} \boldsymbol{x} & =\int_{\mathbb{R}^{q}} f(\boldsymbol{x}) \exp \left\{-0,5 \boldsymbol{x}^{\top} \boldsymbol{x}\right\} \mathrm{d} \boldsymbol{x} \\
& \approx(2 \pi)^{q / 2}|\boldsymbol{\Sigma}|^{-1 / 2} \sum_{i_{1}=1}^{N_{G H}} \ldots \sum_{i_{q}=1}^{N_{G H}} f\left(\boldsymbol{s}^{+}\right) \prod_{k=1}^{q} v_{i_{k}}^{+},
\end{aligned}
$$

em que $\boldsymbol{s}^{+}=\hat{\boldsymbol{\mu}}+\sqrt{2} \boldsymbol{\Sigma}^{-1 / 2} \boldsymbol{s}, \boldsymbol{s}=\left[s_{i_{1}}, \ldots, s_{i_{q}}\right]^{\top}, v_{i_{k}}^{+}=v_{i_{k}} \exp \left\{s_{i_{k}}^{2}\right\} e$

$$
\hat{\boldsymbol{\mu}}=\arg \max _{\boldsymbol{x}} g(\boldsymbol{x}) \quad \text { e } \boldsymbol{\Sigma}=-\left.\frac{\partial^{2} g(\boldsymbol{x})}{\partial \boldsymbol{x} \partial \boldsymbol{x}^{\top}}\right|_{\boldsymbol{x}=\hat{\boldsymbol{\mu}}} .
$$

Observação 6. Quando na equação (4.7) $N_{G H}=1$, temos como caso particular a aproximação de Laplace multidimensional (veja, por exemplo, Pinheiro e Bates, 1995), já que $s_{i}=0$ e $v_{i}=1$, isto é

$$
\int_{\mathbb{R}^{q}} g(\boldsymbol{x}) \mathrm{d} \boldsymbol{x}=\int_{\mathbb{R}^{q}} f(\boldsymbol{x}) \exp \left\{-0,5 \boldsymbol{x}^{\top} \boldsymbol{x}\right\} \mathrm{d} \boldsymbol{x} \approx(2 \pi)^{q / 2}|\boldsymbol{\Sigma}|^{-1 / 2} f(\hat{\boldsymbol{\mu}}),
$$


em que

$$
\hat{\boldsymbol{\mu}}=\arg \max _{\boldsymbol{x}} g(\boldsymbol{x}) \quad e \quad \boldsymbol{\Sigma}=-\left.\frac{\partial^{2} g(\boldsymbol{x})}{\partial \boldsymbol{x} \partial \boldsymbol{x}^{\top}}\right|_{\boldsymbol{x}=\hat{\boldsymbol{\mu}}} .
$$

Mais detalhes da aproximação de Laplace podem ser encontrados em Wolfinger (1993), Vonesh (1992, 1996), Vonesh e Chinchilli (1997) e Wolfinger e Lin (1997).

\subsection{Modelos log-Birnbaum-Saunders mistos}

Seja $Y_{i j}$ o $j$-ésimo logaritmo da resposta associada ao $i$-ésimo grupo (indivíduo), para $i=1, \ldots, n$ e $j=1, \ldots, m_{i}$. Assumimos que o modelo log-Birnbaum-Saunders de efeitos mistos segue a seguinte estrutura hierárquica:

$$
\begin{aligned}
Y_{i j} \mid \boldsymbol{b}_{i} & \stackrel{\text { ind }}{\sim} \log -\operatorname{BS}\left(\alpha, \mu_{i j}\right) \quad \mathrm{e} \\
\boldsymbol{b}_{i} & \stackrel{\text { ind }}{\sim} \mathcal{N}_{q}(\mathbf{0}, \boldsymbol{D}),
\end{aligned}
$$

em que $\mu_{i j}=\boldsymbol{x}_{i j}^{\top} \boldsymbol{\beta}+\boldsymbol{z}_{i j}^{\top} \boldsymbol{b}_{i}, \boldsymbol{x}_{i j}=\left[x_{i j 1}, \ldots, x_{i j p}\right]^{\top}$ contém valores de variáveis explicativas associadas aos efeitos fixos, $\boldsymbol{z}_{i j}=\left[z_{i j 1}, \ldots, z_{i j q}\right]^{\top}$ contém valores de variáveis explicativas associadas aos efeitos aleatórios, $\boldsymbol{\beta}=\left[\beta_{1}, \ldots, \beta_{p}\right]^{\top}$ é um vetor de efeitos fixos (parâmetros desconhecidos), $\boldsymbol{b}_{i}=\left[b_{i 1}, \ldots, b_{i q}\right]^{\top}$ é um vetor de efeitos aleatórios, $\mathbf{0}$ é um vetor de zeros e $\boldsymbol{D}=\boldsymbol{D}(\boldsymbol{\varsigma}) \in \mathbb{R}^{q}$ é a matriz que contém os componentes de variância do modelo cujos elementos são $\varsigma=\left[\varsigma_{11} \ldots \varsigma_{q q}\right]^{\top}$. Seja $\boldsymbol{\theta}=\left[\alpha, \boldsymbol{\beta}^{\top}, \boldsymbol{\varsigma}^{\top}\right]^{\top}$ o vetor de parâmetros a ser estimados no modelo anterior. Usando resultados de distribuições condicionais, temos que

$$
\begin{aligned}
\mathrm{E}\left(Y_{i j}\right) & =\boldsymbol{x}_{i j}^{\top} \boldsymbol{\beta}, \\
\operatorname{Var}\left(Y_{i j}\right) & =4 \omega(\alpha)+\boldsymbol{z}_{i j}^{\top} \boldsymbol{D} \boldsymbol{z}_{i j} \quad \mathrm{e} \\
\operatorname{Cov}\left(Y_{i j}, Y_{i j^{\prime}}\right) & =\boldsymbol{z}_{i j}^{\top} \boldsymbol{D} \boldsymbol{z}_{i j},
\end{aligned}
$$

em que $\omega(\alpha)$ foi definido na equação (1.11). Assim a correlação intraclasse é dada por

$$
\operatorname{Corr}\left(Y_{i j}, Y_{i j^{\prime}}\right)=\frac{\boldsymbol{z}_{i j}^{\top} \boldsymbol{D} \boldsymbol{z}_{i j}}{4 \omega(\alpha)+\boldsymbol{z}_{i j}^{\top} \boldsymbol{D} \boldsymbol{z}_{i j}}
$$

para $i=1, \ldots, n, j=1, \ldots, m_{i}$ e $j \neq j^{\prime}$. A função densidade de probabilidade marginal de $\boldsymbol{Y}=\left[\boldsymbol{Y}_{1}^{\top}, \ldots, \boldsymbol{Y}_{n}^{\top}\right]^{\top}, \operatorname{com} \boldsymbol{Y}_{i}=\left[Y_{i 1}, \ldots, Y_{i m_{i}}\right]^{\top}$, é dada por

$$
f(\boldsymbol{y} ; \boldsymbol{\theta})=\prod_{i=1}^{n} \int_{\mathbb{R}^{q}}\left\{\prod_{j \in O} f_{i j}\left(y_{i j} \mid \mathbf{b}_{i} ; \alpha, \boldsymbol{\beta}\right) \prod_{j \in C} \Phi\left(-\kappa_{i j 2}\right)\right\} f\left(\boldsymbol{b}_{i} ; \boldsymbol{\varsigma}\right) \mathrm{d} \boldsymbol{b}_{i}
$$

com $O$ e $C$ representando os conjuntos associados aos logaritmos dos tempos de falha ou censura, respectivamente, $\Phi(\cdot)$ é a função de distribuição acumulada de $Z \sim \mathcal{N}(0,1)$. A função densidade 
de probabilidade de $Y_{i j} \mid \boldsymbol{b}_{i}$ é dada por

$$
f_{i j}\left(y_{i j} \mid \boldsymbol{b}_{i} ; \alpha, \boldsymbol{\beta}\right)=\exp \left(-\frac{1}{2} \log (8 \pi)+\log \left(\kappa_{i j 1}\right)-\frac{1}{2} \kappa_{i j 2}^{2}\right),
$$

com

$$
\kappa_{i j 1}=\frac{2}{\alpha} \cosh \left(\frac{y_{i j}-\boldsymbol{x}_{i j}^{\top} \boldsymbol{\beta}-\boldsymbol{z}_{i j}^{\top} \boldsymbol{b}_{i}}{2}\right) \quad \text { e } \quad \kappa_{i j 2}=\frac{2}{\alpha} \operatorname{senh}\left(\frac{y_{i j}-\boldsymbol{x}_{i j}^{\top} \boldsymbol{\beta}-\boldsymbol{z}_{i j}^{\top} \boldsymbol{b}_{i}}{2}\right) .
$$

Finalmente, o logaritmo da função de verossimilhança pode ser expresso na forma

$$
L(\boldsymbol{\theta})=\sum_{i=1}^{n} \log \left(\int_{\mathbb{R}^{q}}\left\{\prod_{j \in O} f_{i j}\left(y_{i j} \mid \mathbf{b}_{i} ; \alpha, \boldsymbol{\beta}\right) \prod_{j \in C} \Phi\left(-\kappa_{i j 2}\right)\right\} f\left(\boldsymbol{b}_{i} ; \boldsymbol{\varsigma}\right) \mathrm{d} \boldsymbol{b}_{i}\right) .
$$

Para estimar o vetor de parâmetros $\boldsymbol{\theta}=\left[\alpha, \boldsymbol{\beta}^{\top}, \boldsymbol{\varsigma}^{\top}\right]^{\top}$ no modelo log-Birnbaum-Saunders, usamos a quadratura de Gauss-Hermite não adaptativa para aproximar a função $L(\boldsymbol{\theta})$. O algoritmo quaseNewton implementado no Proc NLMIXED do SAS é utilizado para obter as estimativas de máxima verossimilhança do modelo marginal e os respectivos erros padrão aproximados.

Observação 7. Alguns casos particulares podem ser recuperados da equação (4.8). Por exemplo, considerando dados não censurados e $m_{i}=1$, para todo $i$, tem-se o modelo proposto por Rieck e Nedelman (1991). Considerando dados censurados e $m_{i}=1$, para todo $i$, tem-se o modelo proposto por Leiva et al. (2007). Assim, estendemos esses modelos considerando a inclusão de correlação intraclasse.

Na seguinte seção discutimos o modelo log-Birnbaum-Saunders de intercepto aleatório para dados com censura aleatória, que será aplicado no Capítulo 6.

\subsection{Modelos log-BS de intercepto aleatório}

Suponha que são observados $n$ grupos (por exemplo fábricas), em que cada grupo possui $m_{i}$ observações (por exemplo máquinas dentro das fábricas). Seja $Y_{i j}$ a $j$-ésima medida no $i$-ésimo indivíduo (ou grupo), considerando censura. Vamos supor o modelo por meio da seguinte estrutura hierárquica:

$$
\begin{aligned}
Y_{i j} \mid b_{i} & \stackrel{\text { ind }}{\sim} \log -\mathrm{BS}\left(\alpha, \mu_{i j}\right) \quad \mathrm{e} \\
b_{i} & \stackrel{\text { iid }}{\sim} \mathcal{N}(0, \varsigma), \quad i=1, \ldots, n \quad \text { e } \quad j=1, \ldots, m_{i},
\end{aligned}
$$

em que $\mu_{i j}=\boldsymbol{x}_{i j}^{\top} \boldsymbol{\beta}+b_{i}, \boldsymbol{x}_{i j}=\left[x_{i j 1}, \ldots, x_{i j p}\right]^{\top}$ contém valores das variáveis explicativas associadas aos efeitos fixos, $\boldsymbol{\beta}=\left[\beta_{1}, \ldots, \beta_{p}\right]^{\top}$ contém os parâmetros fixos do modelo e $b_{i}$ é o efeito aleatório associado ao $i$-ésimo grupo. Seja $\boldsymbol{\theta}=\left[\alpha, \boldsymbol{\beta}^{\top}, \varsigma\right]^{\top}$ o vetor de parâmetros a ser estimados no modelo anterior. 


\subsubsection{Consequências do modelo log-BS}

A seguir apresentamos algumas propriedades resultantes do modelo descrito na equação (4.12).

(i) Média de $Y_{i j}$

A média marginal de $Y_{i j}$ pode ser calculada com base na esperança condicional (veja, por exemplo, Casella e Berger, 2002) de $Y_{i j}$ dado $b_{i}$, ou seja,

$$
\mathrm{E}\left[Y_{i j}\right]=\mathrm{E}\left[\mathrm{E}\left(Y_{i j} \mid b_{i}\right)\right]=\boldsymbol{x}_{i j}^{\top} \boldsymbol{\beta}+\mathrm{E}\left[b_{i}\right]=\boldsymbol{x}_{i j}^{\top} \boldsymbol{\beta} .
$$

(ii) Variância de $Y_{i j}$

Para calcular a variância marginal de $Y_{i j}$ usamos a propriedade

$$
\operatorname{Var}[W]=\operatorname{Var}[\mathrm{E}(W \mid X)]+\mathrm{E}[\operatorname{Var}(W \mid X)]
$$

em que $W$ e $X$ são duas variáveis aleatórias (veja, por exemplo, Casella e Berger, 2002). Aplicando a fórmula (4.13) no modelo em questão, temos que

$$
\begin{aligned}
\operatorname{Var}\left[Y_{i j}\right] & =\mathrm{E}\left[\operatorname{Var}\left(Y_{i j} \mid b_{i}\right)\right]+\operatorname{Var}\left[\mathrm{E}\left(Y_{i j} \mid b_{i}\right)\right] \\
& =\mathrm{E}[4 \omega(\alpha)]+\operatorname{Var}\left[\mu_{i j}\right] \\
& =4 \omega(\alpha)+\operatorname{Var}\left[\boldsymbol{x}_{i j}^{\top} \boldsymbol{\beta}+b_{i}\right] \\
& =4 \omega(\alpha)+\operatorname{Var}\left[b_{i}\right] \\
& =4 \omega(\alpha)+\varsigma, \quad \text { em que } \omega(\alpha) \text { foi definido na equação }(1.11)
\end{aligned}
$$

\section{(iii) Covariância e correlação}

Assumindo a independência condicional dos elementos de $Y_{i j}$ e com base nas fórmulas de McCulloch e Searle (2001), temos que

$$
\begin{aligned}
\operatorname{Cov}\left[Y_{i j}, Y_{i j^{\prime}}\right] & =\operatorname{Cov}\left[\mathrm{E}\left(Y_{i j} \mid b_{i}\right), \mathrm{E}\left(Y_{i j^{\prime}} \mid b_{i}\right)\right]+\mathrm{E}\left[\operatorname{Cov}\left(Y_{i j}\left|b_{i}, Y_{i j^{\prime}}\right| b_{i}\right)\right] \\
& =\operatorname{Cov}\left[\boldsymbol{x}_{i j}^{\top} \boldsymbol{\beta}+b_{i}, \boldsymbol{x}_{i j^{\prime}}^{\top} \boldsymbol{\beta}+b_{i}\right] \\
& =\operatorname{Var}\left[b_{i}\right] \\
& =\varsigma, \quad \text { para } j \neq j^{\prime}
\end{aligned}
$$

O parâmetro ऽ representa a covariância intraclasse, ou seja, a covariância entre cada par de observações na mesma classe, e portanto

$$
\operatorname{Corr}\left[Y_{i j}, Y_{i j^{\prime}}\right]=\frac{\varsigma}{4 \omega(\alpha)+\varsigma}
$$

representa a correlação intraclasse. Pode-se observar que, ao introduzir efeitos aleatórios no modelo, introduzimos uma correlação entre as observações, que têm um efeito aleatório em comum. Denotamos a função densidade de probabilidade condicional de $Y_{i j} \mid b_{i}$ por $f_{i j}\left(y_{i j} \mid b_{i} ; \alpha, \boldsymbol{\beta}\right)$ e a 
função densidade de probabilidade de $b_{i}$ por $f\left(b_{i} ; \varsigma\right)$. Daí segue que a função densidade de probabilidade conjunta de $\left(Y_{i j}, b_{i}\right)$ fica dada por

$$
f_{i j}\left(y_{i j}, b_{i} ; \boldsymbol{\theta}\right)=f_{i j}\left(y_{i j} \mid b_{i} ; \alpha, \boldsymbol{\beta}\right) f\left(b_{i} ; \varsigma\right) .
$$

A função densidade de probabilidade conjunta de $\left(\boldsymbol{Y}_{i}, b_{i}\right)$, com $\boldsymbol{Y}_{i}=\left[Y_{i 1}, \ldots, Y_{i m_{i}}\right]^{\top}$, assume a forma

$$
f_{i}\left(\boldsymbol{y}_{i}, b_{i} ; \boldsymbol{\theta}\right)=\left\{\prod_{j \in O} f_{i j}\left(y_{i j} \mid b_{i} ; \alpha, \boldsymbol{\beta}\right) \prod_{j \in C}\left[1-\Phi\left(\kappa_{i j 2}\right)\right]\right\} f\left(b_{i} ; \varsigma\right),
$$

em que $O$ e $C$ representam os conjuntos de logaritmos dos tempos de vida e os logaritmos dos tempos com censura aleatória, respectivamente, $\Phi(\cdot)$ a função de distribuição acumulada de $Z \sim$ $\mathcal{N}(0,1)$. A função densidade de probabilidade de $Y_{i j} \mid b_{i}$ fica dada por

$$
\begin{aligned}
f_{i j}\left(y_{i j} \mid b_{i} ; \alpha, \boldsymbol{\beta}\right) & =\exp \left(-\frac{1}{2} \log (8 \pi)+\log \left(\kappa_{i j 1}\right)-\frac{1}{2} \kappa_{i j 2}^{2}\right), \\
\kappa_{i j 1} & =\frac{2}{\alpha} \cosh \left(\frac{y_{i j}-\boldsymbol{x}_{i j}^{\top} \boldsymbol{\beta}-b_{i}}{2}\right) \text { e } \kappa_{i j 2}=\frac{2}{\alpha} \operatorname{senh}\left(\frac{y_{i j}-\boldsymbol{x}_{i j}^{\top} \boldsymbol{\beta}-b_{i}}{2}\right),
\end{aligned}
$$

em que $\boldsymbol{x}_{i j}^{\top} \boldsymbol{\beta}=\beta_{1} x_{i j 1}+\ldots+\beta_{p} x_{i j p}$. Assim, segue que a função densidade de probabilidade marginal de $\boldsymbol{Y}_{i}$ fica dada por

$$
f_{i}\left(\boldsymbol{y}_{i} ; \alpha, \boldsymbol{\beta}, \varsigma\right)=\int_{-\infty}^{+\infty}\left\{\prod_{j \in O} f_{i j}\left(y_{i j} \mid b_{i} ; \alpha, \boldsymbol{\beta}\right) \prod_{j \in C}\left[1-\Phi\left(\kappa_{i j 2}\right)\right]\right\} f\left(b_{i} ; \varsigma\right) \mathrm{d} b_{i},
$$

que deve ser resolvida numericamente. Finalmente, a função densidade de probabilidade marginal de $\boldsymbol{Y}=\left(\boldsymbol{Y}_{1}^{\top}, \ldots, \boldsymbol{Y}_{n}^{\top}\right)^{\top}$ pode ser escrita na forma

$$
f(\boldsymbol{y} ; \alpha, \boldsymbol{\beta}, \varsigma)=\prod_{i=1}^{n} \int_{-\infty}^{+\infty}\left\{\prod_{j \in O} f_{i j}\left(y_{i j} \mid b_{i} ; \alpha, \boldsymbol{\beta}\right) \prod_{j \in C}\left[1-\Phi\left(\kappa_{i j 2}\right)\right]\right\} f\left(b_{i} ; \varsigma\right) \mathrm{d} b_{i} .
$$

Denotando $\boldsymbol{\theta}=\left[\alpha, \boldsymbol{\beta}^{\top}, \varsigma\right]^{\top}$, o logaritmo da função de verossimilhança pode ser expresso na forma

$$
L(\boldsymbol{\theta})=\sum_{i=1}^{n} \log \left(\int_{-\infty}^{+\infty}\left\{\prod_{j \in O} f_{i j}\left(y_{i j} \mid b_{i} ; \alpha, \boldsymbol{\beta}\right) \prod_{j \in C}\left[1-\Phi\left(\kappa_{i j 2}\right)\right]\right\} f\left(b_{i} ; \varsigma\right) \mathrm{d} b_{i}\right) .
$$

Devido ao envolvimento do efeito aleatório, o logaritmo da função de verossimilhança na equação (4.16), o modelo log-BS de intercepto aleatório deve ser resolvido utilizando integração numérica. Portanto, o problema de otimização ou maximizarão não é simples de se resolver, especialmente quando o número de efeitos aleatórios é grande ( $q \geq 3$ ), já que a função escore e a matriz de informação observada (ou esperada) são difíceis de calcular. Assim os métodos tradicionais de 
otimização numérica como Newton-Raphson ou escore de Fisher não são viáveis de ser utilizados. Ao invés de usar esses métodos, outros métodos podem ser utilizados, em particular o algoritmo de quase-Newton que aproxima numericamente a matriz de segundas derivadas. Uma boa alternativa para obter as estimativas de máxima verossimilhança de $\boldsymbol{\theta}$ é baseada no Proc NLMIXED do SAS, que ajusta modelos não lineares, isto é, modelos nos quais os efeitos fixos e aleatórios são adicionados de forma não linear. Esses modelos têm uma ampla variedade de aplicações, por exemplo na área de farmacocinética. O Proc NLMIXED ajusta modelos não lineares maximizando uma aproximação do logaritmo da função de verossimilhança integrando sobre os efeitos aleatórios. Diversas aproximações da integral estão disponíveis, sendo a principal a quadratura de Gauss-Hermite adaptativa e a aproximação de Taylor de primeira ordem. Há uma variedade de técnicas de otimização disponíveis que podem ser utilizadas, para mais detalhes veja a documentação no manual do usuário SAS/STAT 9.1 (SAS Institute Inc., 2004, Capítulo 51).

Com base no artigo de Tang et al. (2006) vamos aproximar a função escore e a matriz de informação observada, mas usando a quadratura de Gauss-Hermite não adaptativa descrita na Seção 4.1.1. Tang et al. (2006) aproximam a função escore e a matriz de informação observada usando a aproximação de Laplace para modelos de dispersão não lineares mistos e a estimação de parâmetros é feita usando o algoritmo EM (veja, por exemplo Dempster et al., 1977).

\subsubsection{Modelo marginal}

Baseado na estrutura hierárquica do modelo (4.12) e na quadratura de Gauss-Hermite não adaptativa, temos que,

$$
L(\boldsymbol{\theta}) \approx \sum_{i=1}^{n} \log \left(\sum_{k=1}^{q} \frac{v_{k}}{\sqrt{\pi}} A_{i k}(\boldsymbol{\theta}) B_{i k}(\boldsymbol{\theta})\right),
$$

com

$$
\begin{aligned}
& A_{i k}(\boldsymbol{\theta})=\exp \left(\sum_{j \in O}\left[-\frac{1}{2} \log (8 \pi)+\log \left(\xi_{i j k 1}\right)-\frac{1}{2} \xi_{i j k 2}^{2}\right]\right) \mathrm{e} \\
& B_{i k}(\boldsymbol{\theta})=\exp \left(\sum_{j \in C} \log \left(1-\Phi\left(\xi_{i j k 2}\right)\right)\right),
\end{aligned}
$$

em que

$$
\begin{aligned}
& \xi_{i j k 1}=\frac{2}{\alpha} \cosh \left(\frac{y_{i j}-\boldsymbol{x}_{i j}^{\top} \boldsymbol{\beta}-\sqrt{2 \varsigma} s_{k}}{2}\right), \\
& \xi_{i j k 2}=\frac{2}{\alpha} \operatorname{senh}\left(\frac{y_{i j}-\boldsymbol{x}_{i j}^{\top} \boldsymbol{\beta}-\sqrt{2 \varsigma} s_{k}}{2}\right),
\end{aligned}
$$

com $v_{k}$ e $s_{k}$ são o $k$-ésimo peso e o $k$-ésimo zero tabulado do polinômio de Hermite de grau $q$, respectivamente. 


\subsubsection{Função escore}

A função escore é definida como o vetor gradiente de $L(\boldsymbol{\theta})$ em relação a $\boldsymbol{\theta}=\left[\alpha, \boldsymbol{\beta}^{\top}, \varsigma\right]^{\top}$, ou seja, precisamos derivar $L(\boldsymbol{\theta})$ em relação a $\boldsymbol{\theta}$. Portanto, com base na equação (4.17) temos que

$$
\boldsymbol{U}(\boldsymbol{\theta})=\frac{\partial L(\boldsymbol{\theta})}{\partial \boldsymbol{\theta}}=\sum_{i=1}^{n} \frac{\partial \log \left(D_{i}(\boldsymbol{\theta})\right)}{\partial \boldsymbol{\theta}}=\sum_{i=1}^{n} \boldsymbol{U}_{i}(\boldsymbol{\theta}),
$$

em que,

$$
\begin{aligned}
D_{i}(\boldsymbol{\theta}) & =\sum_{k=1}^{q} \frac{v_{k}}{\sqrt{\pi}} A_{i k}(\boldsymbol{\theta}) B_{i k}(\boldsymbol{\theta}) \\
\boldsymbol{U}_{i}(\boldsymbol{\theta}) & =D_{i}^{-1}(\boldsymbol{\theta}) \frac{\partial D_{i}(\boldsymbol{\theta})}{\partial \boldsymbol{\theta}} \text { e } \frac{\partial D_{i}(\boldsymbol{\theta})}{\partial \boldsymbol{\theta}}=\sum_{k=1}^{q} \frac{v_{k}}{\sqrt{\pi}} \frac{\partial\left(A_{i k}(\boldsymbol{\theta}) B_{i k}(\boldsymbol{\theta})\right)}{\partial \boldsymbol{\theta}} .
\end{aligned}
$$

Assim,

$$
\begin{aligned}
C_{i k}(\boldsymbol{\theta}) & =\frac{\partial\left(A_{i k}(\boldsymbol{\theta}) B_{i k}(\boldsymbol{\theta})\right)}{\partial \boldsymbol{\theta}} \\
& =A_{i k}(\boldsymbol{\theta}) B_{i k}(\boldsymbol{\theta})\left(\sum_{j \in O}\left[\xi_{i j k 1}^{-1} \frac{\partial \xi_{i j k 1}}{\partial \boldsymbol{\theta}}-\xi_{i j k 2} \frac{\partial \xi_{i j k 2}}{\partial \boldsymbol{\theta}}\right]-\sum_{j \in C} h\left(\xi_{i j k 2}\right) \frac{\partial \xi_{i j k 2}}{\partial \boldsymbol{\theta}}\right)
\end{aligned}
$$

com

$$
h\left(\xi_{i j k 2}\right)=\frac{\Phi^{\prime}\left(\xi_{i j k 2}\right)}{1-\Phi\left(\xi_{i j k 2}\right)} \quad \text { e } \quad \Phi^{\prime}\left(\xi_{i j k 2}\right)=\frac{\mathrm{d} \Phi\left(\xi_{i j k 2}\right)}{\mathrm{d} \xi_{i j k 2}}=\phi\left(\xi_{i j k 2}\right),
$$

em que $\phi(\cdot)$ e $\Phi(\cdot)$ são, respectivamente, a função densidade de probabilidade e a função de distribuição acumulada da distribuição normal padrão. Logo,

$$
\frac{\partial D_{i}(\boldsymbol{\theta})}{\partial \boldsymbol{\theta}}=\sum_{k=1}^{q} \frac{v_{k}}{\sqrt{\pi}} C_{i k}(\boldsymbol{\theta}) .
$$

Se definirmos $F_{i k}(\boldsymbol{\theta})$ como

$$
F_{i k}(\boldsymbol{\theta})=\sum_{j \in O}\left[\xi_{i j k 1}^{-1} \frac{\partial \xi_{i j k 1}}{\partial \boldsymbol{\theta}}-\xi_{i j k 2} \frac{\partial \xi_{i j k 2}}{\partial \boldsymbol{\theta}}\right]-\sum_{j \in C} h\left(\xi_{i j k 2}\right) \frac{\partial \xi_{i j k 2}}{\partial \boldsymbol{\theta}}
$$

então,

$$
\frac{\partial D_{i}(\boldsymbol{\theta})}{\partial \boldsymbol{\theta}}=\sum_{k=1}^{q} \frac{v_{k}}{\sqrt{\pi}} A_{i k}(\boldsymbol{\theta}) B_{i k}(\boldsymbol{\theta}) F_{i k}(\boldsymbol{\theta}) .
$$

Derivando $L(\boldsymbol{\theta})$ em relação a $\alpha$, temos que

$$
U_{\alpha}(\boldsymbol{\theta})=\sum_{i=1}^{n} D_{i}^{-1}(\boldsymbol{\theta}) \frac{\partial D_{i}(\boldsymbol{\theta})}{\partial \alpha}
$$


com

$$
\frac{\partial D_{i}(\boldsymbol{\theta})}{\partial \alpha}=\sum_{k=1}^{q} \frac{v_{k}}{\sqrt{\pi}} A_{i k}(\boldsymbol{\theta}) B_{i k}(\boldsymbol{\theta}) F_{i k \alpha}(\boldsymbol{\theta})
$$

e

$$
F_{i k \alpha}(\boldsymbol{\theta})=\frac{1}{\alpha} \sum_{j \in O}\left(\xi_{i j k 2}^{2}-1\right)+\frac{1}{\alpha} \sum_{j \in C} h\left(\xi_{i j k 2}\right) \xi_{i j k 2} .
$$

Derivando $L(\boldsymbol{\theta})$ em relação a $\beta_{r}(r=1, \ldots, p)$, temos que

$$
U_{\beta_{r}}(\boldsymbol{\theta})=\sum_{i=1}^{n} D_{i}^{-1}(\boldsymbol{\theta}) \frac{\partial D_{i}(\boldsymbol{\theta})}{\partial \beta_{r}},
$$

com

$$
\begin{aligned}
\frac{\partial D_{i}(\boldsymbol{\theta})}{\partial \beta_{r}} & =\sum_{k=1}^{q} \frac{v_{k}}{\sqrt{\pi}} A_{i k}(\boldsymbol{\theta}) B_{i k}(\boldsymbol{\theta}) F_{i k \beta_{r}}(\boldsymbol{\theta}), \\
F_{i k \beta_{r}}(\boldsymbol{\theta}) & =\frac{1}{2} \sum_{j \in O}\left(\xi_{i j k 1} \xi_{i j k 2}-\xi_{i j k 3}\right) x_{i j r}+\frac{1}{2} \sum_{j \in C} h\left(\xi_{i j k 2}\right) \xi_{i j k 1} x_{i j r}
\end{aligned}
$$

$\mathrm{e}$

$$
\xi_{i j k 3}=\tanh \left(\frac{y_{i j}-\boldsymbol{x}_{i j}^{\top} \boldsymbol{\beta}-\sqrt{2 \varsigma} s_{k}}{2}\right) .
$$

Finalmente derivando $L(\boldsymbol{\theta})$ em relação a $\varsigma$, temos que

$$
U_{\varsigma}(\boldsymbol{\theta})=\sum_{i=1}^{n} D_{i}^{-1}(\boldsymbol{\theta}) \frac{\partial D_{i}(\boldsymbol{\theta})}{\partial \varsigma},
$$

com

$$
\begin{aligned}
& \frac{\partial D_{i}(\boldsymbol{\theta})}{\partial \varsigma}=\sum_{k=1}^{q} \frac{v_{k}}{\sqrt{\pi}} A_{i k}(\boldsymbol{\theta}) B_{i k}(\boldsymbol{\theta}) F_{i k \varsigma}(\boldsymbol{\theta}) \\
& F_{i k \varsigma}(\boldsymbol{\theta})=\frac{1}{\sqrt{8 \varsigma}} \sum_{j \in O}\left(\xi_{i j k 1} \xi_{i j k 2}-\xi_{i j k 3}\right) s_{k}+\frac{1}{\sqrt{8 \varsigma}} \sum_{j \in C} h\left(\xi_{i j k 2}\right) \xi_{i j k 1} s_{k} .
\end{aligned}
$$

Matricialmente $\boldsymbol{U}(\boldsymbol{\theta})$ pode ser expresso na forma

$$
\boldsymbol{U}(\boldsymbol{\theta})=\left[U_{\alpha}(\boldsymbol{\theta}) U_{\beta_{1}}(\boldsymbol{\theta}), \ldots, U_{\beta_{p}}(\boldsymbol{\theta}) U_{\varsigma}(\boldsymbol{\theta})\right]^{\top}
$$


em que,

$$
U_{\alpha}(\theta)=\mathbf{1}^{\top} \boldsymbol{G}_{1} \boldsymbol{G}_{2} \mathbf{1}, \quad U_{\beta_{r}}(\boldsymbol{\theta})=\mathbf{1}^{\top} \boldsymbol{G}_{1} \boldsymbol{G}_{3 r} \mathbf{1}, \quad U_{\varsigma}(\boldsymbol{\theta})=\mathbf{1}^{\top} \boldsymbol{G}_{1} \boldsymbol{G}_{4} \mathbf{1},
$$

com $1 \in \mathbb{R}^{n}$ sendo um vetor de dimensão $n$ com 1 em todas as posições,

$$
\begin{aligned}
\boldsymbol{G}_{1} & =\operatorname{diag}\left\{D_{1}^{-1}(\boldsymbol{\theta}), \ldots, D_{n}^{-1}(\boldsymbol{\theta})\right\}, \\
\boldsymbol{G}_{2} & =\operatorname{diag}\left\{\partial D_{1}(\boldsymbol{\theta}) / \partial \alpha, \ldots, \partial D_{n}(\boldsymbol{\theta}) / \partial \alpha\right\}, \\
\boldsymbol{G}_{3 r} & =\operatorname{diag}\left\{\partial D_{1}(\boldsymbol{\theta}) / \partial \beta_{r}, \ldots, \partial D_{n}(\boldsymbol{\theta}) / \partial \beta_{r}\right\}, \quad r=1, \ldots, p, \quad \mathrm{e} \\
\boldsymbol{G}_{4} & =\operatorname{diag}\left\{\partial D_{1}(\boldsymbol{\theta}) / \partial \varsigma, \ldots, \partial D_{n}(\boldsymbol{\theta}) / \partial \varsigma,\right\} .
\end{aligned}
$$

Para detalhes das derivadas usadas acima, veja Apêndice B.

\subsubsection{Matriz hessiana}

Para obter a matriz hessiana no modelo (4.12), devemos calcular

$$
\begin{aligned}
\ddot{\boldsymbol{L}}_{\theta \theta} & =\sum_{i=1}^{n} \frac{\partial \boldsymbol{U}_{i}^{\top}(\boldsymbol{\theta})}{\partial \boldsymbol{\theta}} \\
& =\sum_{i=1}^{n} \frac{\partial}{\partial \boldsymbol{\theta}}\left\{D_{i}^{-1}(\boldsymbol{\theta}) \frac{\partial D_{i}(\boldsymbol{\theta})}{\partial \boldsymbol{\theta}^{\top}}\right\} \\
& =\sum_{i=1}^{n}\left\{D_{i}^{-1}(\boldsymbol{\theta}) \frac{\partial^{2} D_{i}(\boldsymbol{\theta})}{\partial \boldsymbol{\theta} \partial \boldsymbol{\theta}^{\top}}-D_{i}^{-2}(\boldsymbol{\theta}) \frac{\partial D_{i}(\boldsymbol{\theta})}{\partial \boldsymbol{\theta}} \frac{\partial D_{i}(\boldsymbol{\theta})}{\partial \boldsymbol{\theta}^{\top}}\right\} .
\end{aligned}
$$

Derivação de $\ddot{L}_{\alpha \alpha}$

Com base na equação (4.20), temos que

$$
\ddot{L}_{\alpha \alpha}=\sum_{i=1}^{n}\left\{D_{i}^{-1}(\boldsymbol{\theta}) \frac{\partial^{2} D_{i}(\boldsymbol{\theta})}{\partial^{2} \alpha}-D_{i}^{-2}(\boldsymbol{\theta})\left(\frac{\partial D_{i}(\boldsymbol{\theta})}{\partial \alpha}\right)^{2}\right\}
$$

com

$$
\frac{\partial^{2} D_{i}(\boldsymbol{\theta})}{\partial^{2} \alpha}=\sum_{k=1}^{q} \frac{v_{k}}{\sqrt{\pi}}\left[C_{i k \alpha}(\boldsymbol{\theta}) F_{i k \alpha}(\boldsymbol{\theta})+A_{i k}(\boldsymbol{\theta}) B_{i k}(\boldsymbol{\theta})\left(\frac{\partial F_{i k \alpha}(\boldsymbol{\theta})}{\partial \alpha}\right)\right] .
$$

Logo,

$$
\frac{\partial^{2} D_{i}(\boldsymbol{\theta})}{\partial^{2} \alpha}=\sum_{k=1}^{q} \frac{v_{k}}{\sqrt{\pi}} A_{i k}(\boldsymbol{\theta}) B_{i k}(\boldsymbol{\theta})\left[F_{i k \alpha}^{2}(\boldsymbol{\theta})+\left(\frac{\partial F_{i k \alpha}(\boldsymbol{\theta})}{\partial \alpha}\right)\right],
$$

com

$$
\frac{\partial F_{i k \alpha}(\boldsymbol{\theta})}{\partial \alpha}=\frac{1}{\alpha^{2}}\left(\sum_{j \in O}\left(1-3 \xi_{i j k 2}^{2}\right)-\sum_{j \in C}\left(h^{\prime}\left(\xi_{i j k 2}\right) \xi_{i j k 2}^{2}+2 h\left(\xi_{i j k 2}\right) \xi_{i j k 2}\right)\right)
$$


e

$$
h^{\prime}\left(\xi_{i j k 2}\right)=\frac{\mathrm{d} h\left(\xi_{i j k 2}\right)}{\mathrm{d} \xi_{i j k 2}}=h^{2}\left(\xi_{i j k 2}\right)-\xi_{i j k 2} h\left(\xi_{i j k 2}\right) .
$$

Derivação de $\ddot{L}_{\alpha \beta_{r}}$

Com base na equação (4.20), temos que

$$
\ddot{L}_{\alpha \beta_{r}}=\sum_{i=1}^{n}\left\{D_{i}^{-1}(\boldsymbol{\theta}) \frac{\partial^{2} D_{i}(\boldsymbol{\theta})}{\partial \alpha \partial \beta_{r}}-D_{i}^{-2}(\boldsymbol{\theta}) \frac{\partial D_{i}(\boldsymbol{\theta})}{\partial \alpha} \frac{\partial D_{i}(\boldsymbol{\theta})}{\partial \beta_{r}}\right\}
$$

com

$$
\frac{\partial^{2} D_{i}(\boldsymbol{\theta})}{\partial \alpha \partial \beta_{r}}=\sum_{k=1}^{q} \frac{v_{k}}{\sqrt{\pi}}\left[C_{i k \alpha}(\boldsymbol{\theta}) F_{i k \beta_{r}}(\boldsymbol{\theta})+A_{i k}(\boldsymbol{\theta}) B_{i k}(\boldsymbol{\theta})\left(\frac{\partial F_{i k \beta_{r}}(\boldsymbol{\theta})}{\partial \alpha}\right)\right] .
$$

Logo,

$$
\frac{\partial^{2} D_{i}(\boldsymbol{\theta})}{\partial \alpha \partial \beta_{r}}=\sum_{k=1}^{q} \frac{v_{k}}{\sqrt{\pi}} A_{i k}(\boldsymbol{\theta}) B_{i k}(\boldsymbol{\theta})\left[F_{i k \alpha}(\boldsymbol{\theta}) F_{i k \beta_{r}}(\boldsymbol{\theta})+\left(\frac{\partial F_{i k \beta_{r}}(\boldsymbol{\theta})}{\partial \alpha}\right)\right]
$$

e

$$
\begin{aligned}
\frac{\partial F_{i k \beta_{r}}(\boldsymbol{\theta})}{\partial \alpha}= & -\frac{2}{\alpha^{3}} \sum_{j \in O} \operatorname{senh}\left(y_{i j}-\boldsymbol{x}_{i j}^{\top} \boldsymbol{\beta}-\sqrt{2 \varsigma} s_{k}\right) x_{i j r} \\
& -\frac{1}{2 \alpha} \sum_{j \in C} \xi_{i j k 1}\left(h\left(\xi_{i j k 2}\right) x_{i j r}+\xi_{i j k 2} h^{\prime}\left(\xi_{i j k 2}\right)\right) x_{i j r} .
\end{aligned}
$$

Derivação de $\ddot{L}_{\alpha \varsigma}$

Com base na equação (4.20), temos que

$$
\ddot{L}_{\alpha \varsigma}=\sum_{i=1}^{n}\left\{D_{i}^{-1}(\boldsymbol{\theta}) \frac{\partial^{2} D_{i}(\boldsymbol{\theta})}{\partial \alpha \partial \varsigma}-D_{i}^{-2}(\boldsymbol{\theta}) \frac{\partial D_{i}(\boldsymbol{\theta})}{\partial \alpha} \frac{\partial D_{i}(\boldsymbol{\theta})}{\partial \varsigma}\right\}
$$

com

$$
\frac{\partial^{2} D_{i}(\boldsymbol{\theta})}{\partial \alpha \partial \varsigma}=\sum_{k=1}^{q} \frac{v_{k}}{\sqrt{\pi}}\left[C_{i k \alpha}(\boldsymbol{\theta}) F_{i k \varsigma}(\boldsymbol{\theta})+A_{i k}(\boldsymbol{\theta}) B_{i k}(\boldsymbol{\theta})\left(\frac{\partial F_{i k \varsigma}(\boldsymbol{\theta})}{\partial \alpha}\right)\right] .
$$

Logo,

$$
\frac{\partial^{2} D_{i}(\boldsymbol{\theta})}{\partial \alpha \partial \varsigma}=\sum_{k=1}^{q} \frac{v_{k}}{\sqrt{\pi}} A_{i k}(\boldsymbol{\theta}) B_{i k}(\boldsymbol{\theta})\left[F_{i k \alpha}(\boldsymbol{\theta}) F_{i k \varsigma}(\boldsymbol{\theta})+\left(\frac{\partial F_{i k \varsigma}(\boldsymbol{\theta})}{\partial \alpha}\right)\right],
$$

com

$$
\frac{\partial F_{i k \varsigma}(\boldsymbol{\theta})}{\partial \alpha}=-\frac{s_{k}}{\sqrt{2 \alpha^{2} \varsigma}} \sum_{j \in O} \xi_{i j k 1} \xi_{i j k 2}-\frac{s_{k}}{\sqrt{8 \alpha^{2} \varsigma}} \sum_{j \in C}\left(\xi_{i j k 1} h\left(\xi_{i j k 2}\right)+\xi_{i j k 1} \xi_{i j k 2} h^{\prime}\left(\xi_{i j k 2}\right)\right) .
$$


Derivação de $\ddot{L}_{\beta_{r} \beta_{s}}$

Com base na equação (4.20), temos que

$$
\begin{aligned}
\ddot{L}_{\beta_{r} \beta_{s}} & =\sum_{i=1}^{n}\left\{D_{i}^{-1}(\boldsymbol{\theta}) \frac{\partial^{2} D_{i}(\boldsymbol{\theta})}{\partial \beta_{r} \partial \beta_{s}}-D_{i}^{-2}(\boldsymbol{\theta}) \frac{\partial D_{i}(\boldsymbol{\theta})}{\partial \beta_{r}} \frac{\partial D_{i}(\boldsymbol{\theta})}{\partial \beta_{s}}\right\}, \quad \text { com } \\
\frac{\partial^{2} D_{i}(\boldsymbol{\theta})}{\partial \beta_{r} \partial \beta_{s}} & =\sum_{k=1}^{q} \frac{v_{k}}{\sqrt{\pi}}\left[C_{i k \beta_{r}}(\boldsymbol{\theta}) F_{i k \beta_{s}}(\boldsymbol{\theta})+A_{i k}(\boldsymbol{\theta}) B_{i k}(\boldsymbol{\theta})\left(\frac{\partial F_{i k \beta_{s}}(\boldsymbol{\theta})}{\partial \beta_{r}}\right)\right] .
\end{aligned}
$$

Logo,

$$
\begin{aligned}
\frac{\partial^{2} D_{i}(\boldsymbol{\theta})}{\partial \beta_{r} \partial \beta_{s}}= & \sum_{k=1}^{q} \frac{v_{k}}{\sqrt{\pi}} A_{i k}(\boldsymbol{\theta}) B_{i k}(\boldsymbol{\theta})\left[F_{i k \beta_{r}}(\boldsymbol{\theta}) F_{i k \beta_{s}}(\boldsymbol{\theta})+\left(\frac{\partial F_{i k \beta_{s}}(\boldsymbol{\theta})}{\partial \beta_{r}}\right)\right], \text { com } \\
\frac{\partial F_{i k \beta_{s}}(\boldsymbol{\theta})}{\partial \beta_{r}}= & \frac{1}{4} \sum_{j \in O}\left(\operatorname{sech}^{2}\left(\frac{y_{i j}-\boldsymbol{x}_{i j}^{\top} \boldsymbol{\beta}-\sqrt{2 \varsigma} s_{k}}{2}\right)-\frac{4}{\alpha^{2}} \cosh \left(y_{i j}-\boldsymbol{x}_{i j}^{\top} \boldsymbol{\beta}-\sqrt{2 \varsigma}\right)\right) x_{i j r} x_{i j s} s_{k} \\
& -\frac{1}{4} \sum_{j \in C}\left(\xi_{i j k 1}^{2} h^{\prime}\left(\xi_{i j k 2}\right)+\xi_{i j k 2} h\left(\xi_{i j k 2}\right)\right) x_{i j r} x_{i j s} s_{k} .
\end{aligned}
$$

Derivação de $\ddot{L}_{\varsigma \beta_{r}}$

Com base na equação (4.20), temos que

$$
\begin{aligned}
\ddot{L}_{\varsigma \beta_{r}} & =\sum_{i=1}^{n}\left\{D_{i}^{-1}(\boldsymbol{\theta}) \frac{\partial^{2} D_{i}(\boldsymbol{\theta})}{\partial \varsigma \partial \beta_{r}}-D_{i}^{-2}(\boldsymbol{\theta}) \frac{\partial D_{i}(\boldsymbol{\theta})}{\partial \varsigma} \frac{\partial D_{i}(\boldsymbol{\theta})}{\partial \beta_{r}}\right\}, \quad \text { com } \\
\frac{\partial^{2} D_{i}(\boldsymbol{\theta})}{\partial \varsigma \partial \beta_{r}} & =\sum_{k=1}^{q} \frac{v_{k}}{\sqrt{\pi}}\left[C_{i k \varsigma}(\boldsymbol{\theta}) F_{i k \beta_{r}}(\boldsymbol{\theta})+A_{i k}(\boldsymbol{\theta}) B_{i k}(\boldsymbol{\theta})\left(\frac{\partial F_{i k \beta_{r}}(\boldsymbol{\theta})}{\partial \varsigma}\right)\right] .
\end{aligned}
$$

Logo,

$$
\begin{aligned}
\frac{\partial^{2} D_{i}(\boldsymbol{\theta})}{\partial \varsigma \partial \beta_{r}}= & \sum_{k=1}^{q} \frac{v_{k}}{\sqrt{\pi}} A_{i k}(\boldsymbol{\theta}) B_{i k}(\boldsymbol{\theta})\left[F_{i k \varsigma}(\boldsymbol{\theta}) F_{i k \beta_{r}}(\boldsymbol{\theta})+\left(\frac{\partial F_{i k \beta_{r}}(\boldsymbol{\theta})}{\partial \varsigma}\right)\right], \text { com } \\
\frac{\partial F_{i k \beta_{r}}(\boldsymbol{\theta})}{\partial \varsigma}= & \frac{s_{k}}{\sqrt{32 \varsigma}} \sum_{j \in O}\left(\operatorname{sech}^{2}\left(\frac{y_{i j}-\boldsymbol{x}_{i j}^{\top} \boldsymbol{\beta}-\sqrt{2 \varsigma} s_{k}}{2}\right)-\frac{4}{\alpha^{2}} \cosh \left(y_{i j}-\boldsymbol{x}_{i j}^{\top} \boldsymbol{\beta}-\sqrt{2 \varsigma} s_{k}\right)\right) x_{i j r} \\
& -\frac{s_{k}}{\sqrt{32 \varsigma}} \sum_{j \in C}\left(\xi_{i j k 1}^{2} h^{\prime}\left(\xi_{i j k 2}\right)+\xi_{i j k 2} h\left(\xi_{i j k 2}\right)\right) x_{i j r} .
\end{aligned}
$$

Derivação de $\ddot{L}_{\varsigma \varsigma}$

Com base na equação (4.20), temos que

$$
\begin{aligned}
\ddot{L}_{\varsigma \varsigma} & =\sum_{i=1}^{n}\left\{D_{i}^{-1}(\boldsymbol{\theta}) \frac{\partial^{2} D_{i}(\boldsymbol{\theta})}{\partial^{2} \varsigma}-D_{i}^{-2}(\boldsymbol{\theta})\left(\frac{\partial D_{i}(\boldsymbol{\theta})}{\partial \varsigma}\right)^{2}\right\}, \quad \text { com } \\
\frac{\partial^{2} D_{i}(\boldsymbol{\theta})}{\partial^{2} \varsigma} & =\sum_{k=1}^{q} \frac{v_{k}}{\sqrt{\pi}}\left[C_{i k \varsigma}(\boldsymbol{\theta}) F_{i k \varsigma}(\boldsymbol{\theta})+A_{i k}(\boldsymbol{\theta}) B_{i k}(\boldsymbol{\theta})\left(\frac{\partial F_{i k \varsigma}(\boldsymbol{\theta})}{\partial \varsigma}\right)\right] .
\end{aligned}
$$


Logo,

$$
\begin{aligned}
\frac{\partial^{2} D_{i}(\boldsymbol{\theta})}{\partial^{2} \varsigma}= & \sum_{k=1}^{q} \frac{v_{k}}{\sqrt{\pi}} A_{i k}(\boldsymbol{\theta}) B_{i k}(\boldsymbol{\theta})\left[F_{i k \varsigma}^{2}(\boldsymbol{\theta})+\left(\frac{\partial F_{i k \varsigma}(\boldsymbol{\theta})}{\partial \varsigma}\right)\right], \text { com } \\
\frac{\partial F_{i k \varsigma}(\boldsymbol{\theta})}{\partial \varsigma}= & -\frac{1}{\sqrt{32 \varsigma^{3}}} \sum_{j \in O}\left(\xi_{i j k 1} \xi_{i j k 2}-\xi_{i j k 3}\right) s_{k}-\frac{1}{\sqrt{64 \varsigma^{2}}} \sum_{j \in O}\left(\xi_{i j k 1}^{2}+\xi_{i j k 2}^{2}\right) s_{k}^{2} \\
& +\frac{1}{\sqrt{64 \varsigma^{2}}} \sum_{j \in O} \operatorname{sech}^{2}\left(\frac{y_{i j}-\boldsymbol{x}_{i j}^{\top} \boldsymbol{\beta}-\sqrt{2 \varsigma} s_{k}}{2}\right) s_{k}^{2} \\
& -\frac{1}{\sqrt{32 \varsigma^{3}}} \sum_{j \in C} h\left(\xi_{i j k 2}\right) \xi_{i j k 1} s_{k}-\frac{1}{\sqrt{64 \varsigma^{2}}} \sum_{j \in C}\left(\xi_{i j k 1}^{2} h^{\prime}\left(\xi_{i j k 2}\right)+\xi_{i j k 2} h\left(\xi_{i j k 2}\right)\right) s_{k}^{2} .
\end{aligned}
$$

A matriz hessiana $\ddot{\boldsymbol{L}}_{\theta \theta}$ para o modelo (4.12) pode ser escrita matricialmente como

$$
\ddot{\boldsymbol{L}}_{\theta \theta}=\left[\begin{array}{ccc}
\ddot{L}_{\alpha \alpha} & \ddot{\boldsymbol{L}}_{\alpha \beta} & \ddot{L}_{\alpha \varsigma} \\
\ddot{\boldsymbol{L}}_{\beta \alpha} & \ddot{\boldsymbol{L}}_{\beta \beta} & \ddot{\boldsymbol{L}}_{\beta \varsigma} \\
\ddot{L}_{\varsigma \alpha} & \ddot{\boldsymbol{L}}_{\varsigma \beta} & \ddot{L}_{\varsigma \varsigma}
\end{array}\right],
$$

em que,

$$
\begin{aligned}
\ddot{L}_{\alpha \alpha} & =\mathbf{1}^{\top}\left(\boldsymbol{G}_{1} \boldsymbol{G}_{5}-\boldsymbol{G}_{1}^{2} \boldsymbol{G}_{2}^{2}\right) \mathbf{1}, \ddot{L}_{\alpha \beta_{r}}=\mathbf{1}^{\top}\left(\boldsymbol{G}_{1} \boldsymbol{G}_{6 r}-\boldsymbol{G}_{1}^{2} \boldsymbol{G}_{2} \boldsymbol{G}_{3 r}\right) \mathbf{1}, \\
\ddot{L}_{\alpha \varsigma} & =\mathbf{1}^{\top}\left(\boldsymbol{G}_{1} \boldsymbol{G}_{7}-\boldsymbol{G}_{1}^{2} \boldsymbol{G}_{2} \boldsymbol{G}_{4}\right) \mathbf{1}, \ddot{L}_{\beta_{r} \beta_{s}}=\mathbf{1}^{\top}\left(\boldsymbol{G}_{1} \boldsymbol{G}_{8 r s}-\boldsymbol{G}_{1}^{2} \boldsymbol{G}_{3 r} \boldsymbol{G}_{3 s}\right) \mathbf{1}, \\
\ddot{L}_{\beta_{r} \varsigma} & =\mathbf{1}^{\top}\left(\boldsymbol{G}_{1} \boldsymbol{G}_{9 r}-\boldsymbol{G}_{1}^{2} \boldsymbol{G}_{3 r} \boldsymbol{G}_{4}\right) \mathbf{1}, \ddot{L}_{\varsigma \varsigma}=\mathbf{1}^{\top}\left(\boldsymbol{G}_{1} \boldsymbol{G}_{10}-\boldsymbol{G}_{1}^{2} \boldsymbol{G}_{4}^{2}\right) \mathbf{1} .
\end{aligned}
$$

As matrizes envolvidas são definidas a seguir

$$
\begin{aligned}
& \boldsymbol{G}_{5}=\operatorname{diag}\left\{\frac{\partial^{2} D_{1}(\boldsymbol{\theta})}{\partial^{2} \alpha}, \ldots, \frac{\partial^{2} D_{n}(\boldsymbol{\theta})}{\partial^{2} \alpha}\right\}, \boldsymbol{G}_{6 r}=\operatorname{diag}\left\{\frac{\partial^{2} D_{1}(\boldsymbol{\theta})}{\partial \alpha \partial \beta_{r}}, \ldots, \frac{\partial^{2} D_{n}(\boldsymbol{\theta})}{\partial \alpha \partial \beta_{r}}\right\}, \\
& \boldsymbol{G}_{7}=\operatorname{diag}\left\{\frac{\partial^{2} D_{1}(\boldsymbol{\theta})}{\partial \alpha \partial \varsigma}, \ldots, \frac{\partial^{2} D_{n}(\boldsymbol{\theta})}{\partial \alpha \partial \varsigma}\right\}, \boldsymbol{G}_{8 r s}=\operatorname{diag}\left\{\frac{\partial^{2} D_{1}(\boldsymbol{\theta})}{\partial \beta_{r} \partial \beta_{s}}, \ldots, \frac{\partial^{2} D_{n}(\boldsymbol{\theta})}{\partial \beta_{r} \partial \beta_{s}}\right\}, \\
& \boldsymbol{G}_{9 r}=\operatorname{diag}\left\{\frac{\partial^{2} D_{1}(\boldsymbol{\theta})}{\partial \beta_{r} \partial \varsigma}, \ldots, \frac{\partial^{2} D_{n}(\boldsymbol{\theta})}{\partial \beta_{r} \partial \varsigma}\right\}, \boldsymbol{G}_{10}=\operatorname{diag}\left\{\frac{\partial^{2} D_{1}(\boldsymbol{\theta})}{\partial^{2} \varsigma}, \ldots, \frac{\partial^{2} D_{n}(\boldsymbol{\theta})}{\partial^{2} \varsigma}\right\} .
\end{aligned}
$$

\subsubsection{Estimação dos parâmetros}

Para estimar o vetor de parâmetros $\boldsymbol{\theta}=\left[\alpha, \boldsymbol{\beta}^{\top}, \varsigma\right]^{\top}$ no modelo log-Birnbaum-Saunders de intercepto aleatório, usamos a quadratura de Gauss-Hermite não adaptativa para aproximar a função $L(\boldsymbol{\theta})$ e o algoritmo quase-Newton implementado no Proc NLMIXED do SAS. Para efeitos comparativos, implementou-se computacionalmente o processo de estimação de parâmetros no software R (R Development Core Team, 2008), baseando-se na função optim. Em particular, utilizou-se o pacote glmmML e a função ghq para calcular a quadratura de Gauss-Hermite não adaptativa com $q=50$ pontos de quadratura e o método BFGS (veja Broyden, 1970; Fletcher, 1970; Goldfarb, 1970; Shanno, 1970) para maximizar o logaritmo da função de verossimilhança. Os resultados 
apresentados usando o software $\mathrm{R}$ foram similares aos obtidos usando o Proc NLMIXED do SAS, mas o tempo computational foi consideravelmente maior em R. Portanto, optou-se por usar o Proc NLMIXED do SAS na estimação de parâmetros. Com os resultados obtidos no SAS foram desenvolvidos as análises de diagnósticos, isto é, análises de resíduos e influência local.

\subsubsection{Predição do efeito aleatório}

Um problema frequente de interesse é predizer a variável resposta. Isto é muito importante nas análises de modelos de efeitos aleatórios. Ainda que isto tem sido estudado para os modelos lineares de efeitos aleatórios e modelos lineares generalizados de efeitos aleatórios (veja, por exemplo, Searle et al., 1992; Vonesh e Chinchilli, 1997; McCulloch e Searle, 2001), não há informação sobre como predizer o efeito aleatório no modelo log-Birnbaum-Saunders de efeitos aleatórios. Neste trabalho usamos o estimador de Bayes empírico (veja, por exemplo, Schervish, 1995) para predizer o efeito aleatório, que se define como

$$
\tilde{b}_{i}=\mathrm{E}\left[b_{i} \mid y_{i j} ; \boldsymbol{\theta}\right]=\frac{\int_{-\infty}^{+\infty} b_{i} f\left(y_{i j} \mid b_{i} ; \alpha, \boldsymbol{\beta}\right) f\left(b_{i} ; \varsigma\right) \mathrm{d} b_{i}}{\int_{-\infty}^{+\infty} f\left(y_{i j} \mid b_{i} ; \alpha, \boldsymbol{\beta}\right) f\left(b_{i} ; \varsigma\right) \mathrm{d} b_{i}} .
$$

Utilizamos o Proc NLMIXED do SAS para calcular $\tilde{b}_{i}$.

\subsubsection{Teste do componente de variância}

Em geral, para testar a hipótese

$$
H_{0}: \varsigma=0 \quad \text { vs } \quad H_{1}: \varsigma>0
$$

no modelo log-Birnbaum-Saunders de intercepto aleatório, podemos usar o teste da razão de verossimilhanças (RV) dado por

$$
\mathrm{RV}=2[L(\hat{\alpha}, \hat{\boldsymbol{\beta}}, \hat{\varsigma})-L(\tilde{\alpha}, \tilde{\boldsymbol{\beta}}, \varsigma=0)]
$$

em que $[\hat{\alpha}, \hat{\boldsymbol{\beta}}, \hat{\varsigma}]^{\top}$ e $[\tilde{\alpha}, \tilde{\boldsymbol{\beta}}, 0]^{\top}$, são respectivamente, as estimativas de máxima verossimilhança no modelo log-Birnbaum-Saunders com intercepto aleatório sob as hipóteses $H_{1}$ e $H_{0}$. Como sob a hipótese nula o teste RV está na fronteira do espaço paramétrico para $\varsigma$, a estatística RV não segue para $n$ grande uma distribuição nula $\chi_{1}^{2}$.

Usando os resultados de Self e Liang (1987), Stram e Lee (1994) mostraram que para testar o componente de variância no modelo de intercepto aleatório linear normal a estatística RV segue para $n$ grande e sob $H_{0}$ uma mistura de distribuições qui-quadrado, isto é

$$
\mathrm{RV} \sim \frac{1}{2} \chi_{0}^{2}+\frac{1}{2} \chi_{1}^{2}
$$

em que $\chi_{0}^{2}$ representa a distribuição degenerada na origem, isto é $P\left(\chi_{0}^{2}=0\right)=1$. No trabalho de Self e Liang (1987), se apresenta a distribuição assintótica do teste RV baseado na suposição de que as variáveis são independentes e identicamente distribuídas, Vu e Zhou (1997) estenderam o 
trabalho desses autores para caso em que as variáveis não são identicamente distribuídas. Recentemente, Giampaoli e Singer (2009) discutem o artigo de Stram e Lee (1994) e usam o trabalho de Vu e Zhou (1997) para estudar o comportamento assintótico do teste RV para modelos lineares mistos. Giampaoli e Singer (2009) mostram que os resultados de Stram e Lee (1994), com base no artigo de Self e Liang (1987) são válidos, mas que em geral a suposição de variáveis aleatórias identicamente distribuídas no contexto de dados longitudinais não são sempre verdadeiras.

Alternativamente, outros trabalhos têm sido desenvolvidos para testar o componente de variância, em particular baseado no trabalho de Silvapulle e Silvapulle (1995), Verbeke e Molenberghs (2003) aplicam o teste tipo escore para modelos lineares mistos. A estatística do tipo escore apresenta a grande vantagem de requerer que o modelo seja estimado somente sob a hipótese nula. Savalli et al. (2006) e Russo (2010) aplicam a estatística do tipo escore a modelos lineares elípticos mistos e modelos não lineares elípticos mistos, respectivamente.

\subsubsection{Influência local dos grupos}

Os esquemas de perturbação considerados aqui são: ponderação de casos, perturbação da resposta e perturbação de variáveis explicativas contínuas. Lembrando que a matriz $\boldsymbol{\Delta}$ de perturbações (Seção 3.15) foi definida como

$$
\boldsymbol{\Delta}=\frac{\partial^{2} L(\boldsymbol{\theta} \mid \boldsymbol{\omega})}{\partial \boldsymbol{\theta} \partial \boldsymbol{\omega}^{\top}} \quad \text { sendo avaliada em } \boldsymbol{\theta}=\hat{\boldsymbol{\theta}} \text { e } \boldsymbol{\omega}=\boldsymbol{\omega}_{0} .
$$

Vamos definir $\boldsymbol{\Delta}_{i}$ como a $i$-ésima coluna de $\boldsymbol{\Delta}$, isto é

$$
\boldsymbol{\Delta}_{i}=\frac{\partial^{2} L(\boldsymbol{\theta} \mid \boldsymbol{\omega})}{\partial \omega_{i} \partial \boldsymbol{\theta}}, \quad i=1, \ldots, n
$$

em que $\boldsymbol{\theta}=[\alpha, \boldsymbol{\beta}, \varsigma]^{\top}$. Baseando-se na equação (4.23), podemos reescrever $\boldsymbol{\Delta}_{i}$ como

$$
\boldsymbol{\Delta}_{i}=\left[\Delta_{i \alpha}, \boldsymbol{\Delta}_{i \beta}, \Delta_{i \varsigma}\right]^{\top}, \quad i=1, \ldots, n,
$$

em que,

$$
\begin{aligned}
\Delta_{i \alpha} & =\left.\frac{\partial^{2} L(\boldsymbol{\theta} \mid \boldsymbol{\omega})}{\partial \omega_{i} \partial \alpha}\right|_{\boldsymbol{\theta}=\hat{\boldsymbol{\theta}}, \boldsymbol{\omega}=\boldsymbol{\omega}_{0}}, \\
\boldsymbol{\Delta}_{i \beta} & =\left.\frac{\partial^{2} L(\boldsymbol{\theta} \mid \boldsymbol{\omega})}{\partial \omega_{i} \partial \boldsymbol{\beta}}\right|_{\boldsymbol{\theta}=\hat{\boldsymbol{\theta}}, \boldsymbol{\omega}=\boldsymbol{\omega}_{0}}
\end{aligned}
$$

e

$$
\Delta_{i \varsigma}=\left.\frac{\partial^{2} L(\boldsymbol{\theta} \mid \boldsymbol{\omega})}{\partial \omega_{i} \partial \varsigma}\right|_{\boldsymbol{\theta}=\hat{\boldsymbol{\theta}}, \boldsymbol{\omega}=\boldsymbol{\omega}_{0}},
$$

com $\boldsymbol{\Delta}_{i \beta}=\left[\Delta_{i \beta_{1}}, \ldots, \Delta_{i \beta_{p}}\right]^{\top}$. Finalmente $\boldsymbol{\Delta}=\left[\boldsymbol{\Delta}_{1}, \ldots, \boldsymbol{\Delta}_{n}\right]$. 


\section{Ponderação de casos}

Com este tipo de perturbação se deseja avaliar se a contribuição das observações com ponderações diferentes afetam a estimativa de máxima verossimilhança de $\boldsymbol{\theta}$. Segundo Cook (1987), a perturbação de ponderação de casos, é talvez o método mais comum, para avaliar a influência de uma modificação pequena num modelo. Se consideramos o modelo postulado como sendo um modelo log-Birnbaum-Saunders de intercepto aleatório temos que

$$
L(\boldsymbol{\theta})=\sum_{i=1}^{n} \log \left(D_{i}(\boldsymbol{\theta})\right) .
$$

Então, o logaritmo da função de verossimilhança do modelo perturbado fica dado por

$$
L(\boldsymbol{\theta} \mid \boldsymbol{\omega})=\sum_{i=1}^{n} \omega_{i} L_{i}(\boldsymbol{\theta}),
$$

em que $\boldsymbol{\omega}=\left[\omega_{1}, \ldots, \omega_{n}\right]^{\top}$ e $\omega_{i}>0$ representa uma ponderação fixa para a $i$-ésima observação. Nesse caso, quando $\boldsymbol{\omega}=\boldsymbol{\omega}_{0}=[1, \ldots, 1]^{\top}$, o modelo perturbado se reduz ao modelo postulado, isto é, $L(\boldsymbol{\theta})=L(\boldsymbol{\theta} \mid \boldsymbol{\omega})$. Portanto, derivando a equação (4.24) em relação a $\omega_{i}$, tem-se que

$$
\frac{\partial L(\boldsymbol{\theta} \mid \boldsymbol{\omega})}{\partial \omega_{i}}=L_{i}(\boldsymbol{\theta})
$$

Assim, matricialmente $\Delta_{i \gamma}=\left[\Delta_{i \alpha}, \Delta_{i \beta_{r}}, \Delta_{i \varsigma}\right]^{\top}$ pode ser escrita como

$$
\begin{aligned}
\boldsymbol{\Delta}_{\alpha} & =\left.\frac{\partial^{2} L(\boldsymbol{\theta} \mid \boldsymbol{\omega})}{\partial \alpha \partial \boldsymbol{\omega}^{\top}}\right|_{\boldsymbol{\theta}=\hat{\boldsymbol{\theta}}, \boldsymbol{\omega}=\boldsymbol{\omega}_{0}}=\left(\boldsymbol{g}_{1} \odot \boldsymbol{g}_{2}\right)^{\top} \\
\boldsymbol{\Delta}_{\beta_{r}} & =\left.\frac{\partial^{2} L(\boldsymbol{\theta} \mid \boldsymbol{\omega})}{\partial \beta_{r} \partial \boldsymbol{\omega}^{\top}}\right|_{\boldsymbol{\theta}=\hat{\boldsymbol{\theta}}, \boldsymbol{\omega}=\boldsymbol{\omega}_{0}}=\left(\boldsymbol{g}_{1} \odot \boldsymbol{g}_{3 r}\right)^{\top}, \quad r=1, \ldots, p \\
\boldsymbol{\Delta}_{\varsigma} & =\left.\frac{\partial^{2} L(\boldsymbol{\theta} \mid \boldsymbol{\omega})}{\partial \varsigma \partial \boldsymbol{\omega}^{\top}}\right|_{\boldsymbol{\theta}=\hat{\boldsymbol{\theta}}, \boldsymbol{\omega}_{=\boldsymbol{\omega}_{0}}=\left(\boldsymbol{g}_{1} \odot \boldsymbol{g}_{4}\right)^{\top},}
\end{aligned}
$$

em que $\odot$ denota o produto Hadamard (veja, por exemplo, Ravishanker e Dipak, 2002),

$$
\begin{aligned}
\boldsymbol{g}_{1} & =\left(D_{1}^{-1}(\boldsymbol{\theta}), \ldots, D_{n}^{-1}(\boldsymbol{\theta})\right)^{\top} \\
\boldsymbol{g}_{2} & =\left(\partial D_{1}(\boldsymbol{\theta}) / \partial \alpha, \ldots, \partial D_{n}(\boldsymbol{\theta}) / \partial \alpha\right)^{\top}, \\
\boldsymbol{g}_{3 r} & =\left(\partial D_{1}(\boldsymbol{\theta}) / \partial \beta_{r}, \ldots, \partial D_{n}(\boldsymbol{\theta}) / \partial \beta_{r}\right)^{\top} r=1, \ldots, p \quad \mathrm{e} \\
\boldsymbol{g}_{4} & =\left(\partial D_{1}(\boldsymbol{\theta}) / \partial \varsigma, \ldots, \partial D_{n}(\boldsymbol{\theta}) / \partial \varsigma\right)^{\top} .
\end{aligned}
$$

\section{Perturbação dos dados}

Billor e Loynes (1993) consideram que os tipos de perturbação possíveis dependem do modelo. Há duas razões para considerar perturbação de dados: a presença de medidas com erros de medição e a presença de observações aberrantes (em uma proporção relativamente pequena dos dados). 


\section{Perturbação aditiva da resposta}

Seja $\boldsymbol{\omega}=\left[\omega_{1}, \ldots, \omega_{n}\right]^{\top}$ o vetor de perturbações do modelo. Então vamos perturbar os valores da variável resposta da seguinte forma: $y_{i j}\left(\omega_{i}\right)=y_{i j}+\omega_{i}$, em que $\omega_{i}$ é o $i$-ésimo elemento do vetor $\boldsymbol{\omega}$. Nesse caso, quando $\boldsymbol{\omega}=\boldsymbol{\omega}_{0}=[0, \ldots, 0]^{\top}$, o modelo perturbado se reduz ao modelo postulado, isto é, $L(\boldsymbol{\theta})=L(\boldsymbol{\theta} \mid \boldsymbol{\omega})$, em que

$$
\begin{aligned}
L(\boldsymbol{\theta} \mid \boldsymbol{\omega}) & =\sum_{i=1}^{n} \log \left(D_{\omega_{i 1}}(\boldsymbol{\theta})\right), \\
D_{\omega_{i 1}}(\boldsymbol{\theta}) & =\sum_{k=1}^{q} \frac{v_{k}}{\sqrt{\pi}} A_{\omega_{i 1 k}}(\boldsymbol{\theta}) B_{\omega_{i 1 k}}(\boldsymbol{\theta}), \\
A_{\omega_{i 1 k}}(\boldsymbol{\theta}) & =\exp \left(\sum_{j \in O}\left[-\frac{1}{2} \log (8 \pi)+\log \left(\xi_{i j k 1}\left(\omega_{i}\right)\right)-\frac{1}{2} \xi_{i j k 2}^{2}\left(\omega_{i}\right)\right]\right), \quad \mathrm{e} \\
B_{\omega_{i 1 k}}(\boldsymbol{\theta}) & =\exp \left(\sum_{j \in C} \log \left(1-\Phi\left(\xi_{i j k 2}\left(\omega_{i}\right)\right)\right)\right),
\end{aligned}
$$

com

$$
\xi_{i j k 1}\left(\omega_{i}\right)=\frac{2}{\alpha} \cosh \left(\frac{y_{i j}\left(\omega_{i}\right)-\boldsymbol{x}_{i j}^{\top} \boldsymbol{\beta}-\sqrt{2 \varsigma} s_{k}}{2}\right)
$$

e

$$
\xi_{i j k 2}\left(\omega_{i}\right)=\frac{2}{\alpha} \operatorname{senh}\left(\frac{y_{i j}\left(\omega_{i}\right)-\boldsymbol{x}_{i j}^{\top} \boldsymbol{\beta}-\sqrt{2 \varsigma} s_{k}}{2}\right) .
$$

A matriz $\boldsymbol{\Delta}_{i \gamma}, \gamma=\left[\alpha, \beta_{r}, \varsigma\right]^{\top}$, pode ser escrita como

$$
\boldsymbol{\Delta}_{i \gamma}=\frac{\partial^{2} L(\boldsymbol{\theta} \mid \boldsymbol{\omega})}{\partial \omega_{i} \partial \boldsymbol{\gamma}}=\frac{\partial}{\partial \omega_{i}}\left(\frac{\partial L(\boldsymbol{\theta} \mid \boldsymbol{\omega})}{\partial \boldsymbol{\gamma}}\right)=\frac{\partial}{\partial \omega_{i}}\left(\sum_{i=1}^{n} D_{\omega_{i 1}}^{-1}(\boldsymbol{\theta}) \frac{\partial D_{\omega_{i 1}}(\boldsymbol{\theta})}{\partial \boldsymbol{\gamma}}\right) .
$$

Então,

$$
\boldsymbol{\Delta}_{i \gamma}=D_{\omega_{i 1}}^{-1}(\boldsymbol{\theta}) \frac{\partial^{2} D_{\omega_{i 1}}(\boldsymbol{\theta})}{\partial \omega_{i} \partial \gamma}-D_{\omega_{i 1}}^{-2}(\boldsymbol{\theta}) \frac{\partial D_{\omega_{i 1}}(\boldsymbol{\theta})}{\partial \omega_{i}} \frac{\partial D_{\omega_{i 1}}(\boldsymbol{\theta})}{\partial \boldsymbol{\gamma}} .
$$

Se definimos, $b_{i 1}=D_{\omega_{i 1}}^{-1}(\boldsymbol{\theta}), c_{i 1}=\frac{\partial D_{\omega_{i 1}}(\boldsymbol{\theta})}{\partial \omega_{i}}$ e $\boldsymbol{d}_{i 1 \gamma}=\frac{\partial D_{\omega_{i 1}}(\boldsymbol{\theta})}{\partial \boldsymbol{\gamma}}$, temos que

$$
\boldsymbol{\Delta}_{i \gamma}=b_{i 1}\left(\frac{\partial \boldsymbol{d}_{i 1 \gamma}}{\partial \omega_{i}}-b_{i 1} c_{i 1} \boldsymbol{d}_{i 1 \gamma}\right) .
$$

Em primeiro lugar, vamos calcular $c_{i 1}$

$$
c_{i 1}=\sum_{k=1}^{q} \frac{v_{k}}{\sqrt{\pi}} A_{\omega_{i 1 k}}(\boldsymbol{\theta}) B_{\omega_{i 1 k}}(\boldsymbol{\theta}) F\left(y_{i j}\left(\omega_{i}\right)\right)
$$


em que,

$$
\begin{aligned}
F\left(y_{i j}\left(\omega_{i}\right)\right) & =\sum_{j \in O}\left[\xi_{i j k 1}^{-1}\left(\omega_{i}\right) \frac{\partial \xi_{i j k 1}\left(\omega_{i}\right)}{\partial \omega_{i}}-\xi_{i j k 2}\left(\omega_{i}\right) \frac{\partial \xi_{i j k 2}\left(\omega_{i}\right)}{\partial \omega_{i}}\right]-\sum_{j \in C} h\left(\xi_{i j k 2}\left(\omega_{i}\right)\right) \frac{\partial \xi_{i j k 2}\left(\omega_{i}\right)}{\partial \omega_{i}} \\
& =\frac{1}{2} \sum_{j \in O}\left[\xi_{i j k 3}\left(\omega_{i}\right)-\xi_{i j k 2}\left(\omega_{i}\right) \xi_{i j k 1}\left(\omega_{i}\right)\right]-\frac{1}{2} \sum_{j \in C} h\left(\xi_{i j k 2}\left(\omega_{i}\right)\right) \xi_{i j k 1}\left(\omega_{i}\right) \quad \mathrm{e} \\
\xi_{i j k 3}\left(\omega_{i}\right) & =\tanh \left(\frac{y_{i j}\left(\omega_{i}\right)-\boldsymbol{x}_{i j}^{\top} \boldsymbol{\beta}-\sqrt{2 \varsigma} s_{k}}{2}\right) .
\end{aligned}
$$

Por outro lado,

$$
\boldsymbol{d}_{i 1 \gamma}=\sum_{k=1}^{q} \frac{v_{k}}{\sqrt{\pi}} A_{\omega_{i 1 k}}(\boldsymbol{\theta}) B_{\omega_{i 1 k}}(\boldsymbol{\theta}) \boldsymbol{F}_{\omega_{i 1 k \gamma}}(\boldsymbol{\theta}),
$$

com

$$
\boldsymbol{F}_{\omega_{i 1 k \gamma}}(\boldsymbol{\theta})=\sum_{j \in O}\left[\xi_{i j k 1}^{-1}\left(\omega_{i}\right) \frac{\partial \xi_{i j k 1}\left(\omega_{i}\right)}{\partial \boldsymbol{\gamma}}-\xi_{i j k 2}\left(\omega_{i}\right) \frac{\partial \xi_{i j k 2}\left(\omega_{i}\right)}{\partial \boldsymbol{\gamma}}\right]-\sum_{j \in C} h\left(\xi_{i j k 2}\left(\omega_{i}\right)\right) \frac{\partial \xi_{i j k 2}\left(\omega_{i}\right)}{\partial \boldsymbol{\gamma}} .
$$

Em segundo lugar,

$$
\frac{\partial \boldsymbol{d}_{i 1 \gamma}}{\partial \omega_{i}}=\sum_{k=1}^{q} \frac{v_{k}}{\sqrt{\pi}}\left[\frac{\partial\left(A_{\omega_{i 1 k}}(\boldsymbol{\theta}) B_{\omega_{i 1 k}}(\boldsymbol{\theta})\right)}{\partial \omega_{i}} \boldsymbol{F}_{\omega_{i 1 k \gamma}}(\boldsymbol{\theta})+A_{\omega_{i 1 k}}(\boldsymbol{\theta}) B_{\omega_{i 1 k}}(\boldsymbol{\theta}) \frac{\partial \boldsymbol{F}_{\omega_{i 1 k \gamma}}(\boldsymbol{\theta})}{\partial \omega_{i}}\right],
$$

com

$$
\begin{aligned}
\frac{\partial\left(A_{\omega_{i 1 k}}(\boldsymbol{\theta}) B_{\omega_{i 1 k}}(\boldsymbol{\theta})\right)}{\partial \omega_{i}} & =A_{\omega_{i 1 k}}(\boldsymbol{\theta}) B_{\omega_{i 1 k}}(\boldsymbol{\theta}) F\left(y_{i j}\left(\omega_{i}\right)\right) \mathrm{e} \\
\frac{\partial \boldsymbol{F}_{\omega_{i 1 k \gamma}}(\boldsymbol{\theta})}{\partial \omega_{i}} & =\left[\frac{\partial F_{\omega_{i 1 k \alpha}}(\boldsymbol{\theta})}{\partial \omega_{i}}, \frac{\partial F_{\omega_{i 1 k \beta_{r}}}(\boldsymbol{\theta})}{\partial \omega_{i}}, \frac{\partial F_{\omega_{i 1 k \varsigma}}(\boldsymbol{\theta})}{\partial \omega_{i}}\right]^{\top}
\end{aligned}
$$

Portanto,

$$
\frac{\partial \boldsymbol{d}_{i 1 \gamma}}{\partial \omega_{i}}=\sum_{k=1}^{q} \frac{v_{k}}{\sqrt{\pi}} A_{\omega_{i 1 k}}(\boldsymbol{\theta}) B_{\omega_{i 1 k}}(\boldsymbol{\theta})\left[F\left(y_{i j}\left(\omega_{i}\right)\right) \boldsymbol{F}_{\omega_{i 1 k \gamma}}(\boldsymbol{\theta})+\frac{\partial \boldsymbol{F}_{\omega_{i 1 k \gamma}}(\boldsymbol{\theta})}{\partial \omega_{i}}\right]
$$

\section{Derivando $\Delta_{i \alpha}$}

Com base na equação (4.25) temos que

$$
\Delta_{i \alpha}=b_{i 1}\left(\frac{\partial d_{i 1 \alpha}}{\partial \omega_{i}}-b_{i 1} c_{i 1} d_{i 1 \alpha}\right),
$$

em que,

$$
d_{i 1 \alpha}=\sum_{k=1}^{q} \frac{v_{k}}{\sqrt{\pi}} A_{\omega_{i 1 k}}(\boldsymbol{\theta}) B_{\omega_{i 1 k}}(\boldsymbol{\theta}) F_{\omega_{i 1 k \alpha}}(\boldsymbol{\theta}),
$$


com

$$
F_{\omega_{i 1 k \alpha}}(\boldsymbol{\theta})=\frac{1}{\alpha} \sum_{j \in O}\left(\xi_{i j k 2}^{2}\left(\omega_{i}\right)-1\right)+\frac{1}{\alpha} \sum_{j \in C} h\left(\xi_{i j k 2}\left(\omega_{i}\right)\right) \xi_{i j k 2}\left(\omega_{i}\right) .
$$

Com base na equação (4.26), temos que

$$
\frac{\partial d_{i 1 \alpha}}{\partial \omega_{i}}=\sum_{k=1}^{q} \frac{v_{k}}{\sqrt{\pi}} A_{\omega_{i 1 k}}(\boldsymbol{\theta}) B_{\omega_{i 1 k}}(\boldsymbol{\theta})\left[F\left(y_{i j}\left(\omega_{i}\right)\right) F_{\omega_{i 1 k \alpha}}(\boldsymbol{\theta})+\frac{\partial F_{\omega_{i 1 k \alpha}}(\boldsymbol{\theta})}{\partial \omega_{i}}\right]
$$

e

$$
\frac{\partial F_{\omega_{i 1 k \alpha}}(\boldsymbol{\theta})}{\partial \omega_{i}}=\frac{1}{2 \alpha}\left[\sum_{j \in O} 2 \xi_{i j k 2}\left(\omega_{i}\right) \xi_{i j k 1}\left(\omega_{i}\right)+\sum_{j \in C} \xi_{i j k 1}\left(\omega_{i}\right)\left(h^{\prime}\left(\xi_{i j k 2}\left(\omega_{i}\right)\right) \xi_{i j k 2}\left(\omega_{i}\right)+h\left(\xi_{i j k 2}\left(\omega_{i}\right)\right)\right)\right] .
$$

Derivando $\Delta_{i \beta_{r}}$

Com base na equação (4.25) temos que

$$
\Delta_{i \beta_{r}}=b_{i 1}\left(\frac{\partial d_{i 1 \beta_{r}}}{\partial \omega_{i}}-b_{i 1} c_{i 1} d_{i 1 \beta_{r}}\right),
$$

em que

$$
d_{i 1 \beta_{r}}=\sum_{k=1}^{q} \frac{v_{k}}{\sqrt{\pi}} A_{\omega_{i 1 k}}(\boldsymbol{\theta}) B_{\omega_{i 1 k}}(\boldsymbol{\theta}) F_{\omega_{i 1 k \beta_{r}}}(\boldsymbol{\theta}),
$$

com

$$
F_{\omega_{i 1 k \beta_{r}}}(\boldsymbol{\theta})=\frac{1}{2} \sum_{j \in O}\left(\xi_{i j k 1}\left(\omega_{i}\right) \xi_{i j k 2}\left(\omega_{i}\right)-\xi_{i j k 3}\left(\omega_{i}\right)\right) x_{i j r}+\frac{1}{2} \sum_{j \in C} h\left(\xi_{i j k 2}\left(\omega_{i}\right)\right) \xi_{i j k 1}\left(\omega_{i}\right) x_{i j r} .
$$

Com base na equação (4.26), temos que

$$
\frac{\partial d_{i 1 \beta_{r}}}{\partial \omega_{i}}=\sum_{k=1}^{q} \frac{v_{k}}{\sqrt{\pi}} A_{\omega_{i 1 k}}(\boldsymbol{\theta}) B_{\omega_{i 1 k}}(\boldsymbol{\theta})\left[F\left(y_{i j}\left(\omega_{i}\right)\right) F_{\omega_{i 1 k \beta_{r}}}(\boldsymbol{\theta})+\frac{\partial F_{\omega_{i 1 k \beta_{r}}}(\boldsymbol{\theta})}{\partial \omega_{i}}\right]
$$

e

$$
\begin{aligned}
\frac{\partial F_{\omega_{i 1 k \beta_{r}}}(\boldsymbol{\theta})}{\partial \omega_{i}} & =\frac{1}{4} \sum_{j \in O}\left(\xi_{i j k 1}^{2}\left(\omega_{i}\right)+\xi_{i j k 2}^{2}\left(\omega_{i}\right)-\left(1-\xi_{i j k 3}^{2}\left(\omega_{i}\right)\right)\right) x_{i j r} \\
& +\frac{1}{4} \sum_{j \in C}\left(h^{\prime}\left(\xi_{i j k 2}\left(\omega_{i}\right)\right) \xi_{i j k 1}^{2}\left(\omega_{i}\right)+h\left(\xi_{i j k 2}\left(\omega_{i}\right)\right) \xi_{i j k 2}\left(\omega_{i}\right)\right) x_{i j r} .
\end{aligned}
$$

Derivando $\Delta_{i \varsigma}$

Com base na equação (4.25), temos que

$$
\Delta_{i \varsigma}=b_{i 1}\left(\frac{\partial d_{i 1 \varsigma}}{\partial \omega_{i}}-b_{i 1} c_{i 1} d_{i 1 \varsigma}\right),
$$


em que,

$$
d_{i 1 \varsigma}=\sum_{k=1}^{q} \frac{v_{k}}{\sqrt{\pi}} A_{\omega_{i 1 k}}(\boldsymbol{\theta}) B_{\omega_{i 1 k}}(\boldsymbol{\theta}) F_{\omega_{i 1 k \varsigma}}(\boldsymbol{\theta}),
$$

com

$$
F_{\omega_{i 1 k \varsigma}}(\boldsymbol{\theta})=\frac{1}{\sqrt{8 \varsigma}} \sum_{j \in O}\left(\xi_{i j k 1}\left(\omega_{i}\right) \xi_{i j k 2}\left(\omega_{i}\right)-\xi_{i j k 3}\left(\omega_{i}\right)\right) s_{k}+\frac{1}{\sqrt{8 \varsigma}} \sum_{j \in C} h\left(\xi_{i j k 2}\left(\omega_{i}\right)\right) \xi_{i j k 1}\left(\omega_{i}\right) s_{k} .
$$

Com base na equação (4.26), temos que

$$
\begin{aligned}
& \frac{\partial d_{i 1 \varsigma}}{\partial \omega_{i}}=\sum_{k=1}^{q} \frac{v_{k}}{\sqrt{\pi}} A_{\omega_{i 1 k}}(\boldsymbol{\theta}) B_{\omega_{i 1 k}}(\boldsymbol{\theta})\left[F\left(y_{i j}\left(\omega_{i}\right)\right) F_{\omega_{i 1 k \varsigma}}(\boldsymbol{\theta})+\frac{\partial F_{\omega_{i 1 k \varsigma}}(\boldsymbol{\theta})}{\partial \omega_{i}}\right] \mathrm{e} \\
& \frac{\partial F_{\omega_{i 1 k \varsigma}}(\boldsymbol{\theta})}{\partial \omega_{i}}=\frac{1}{\sqrt{32 \varsigma}} \sum_{j \in O}\left(\xi_{i j k 1}^{2}\left(\omega_{i}\right)+\xi_{i j k 2}^{2}\left(\omega_{i}\right)-\left(1-\xi_{i j k 3}^{2}\left(\omega_{i}\right)\right)\right) s_{k} \\
& +\frac{1}{\sqrt{32 \varsigma}} \sum_{j \in C}\left(h^{\prime}\left(\xi_{i j k 2}\left(\omega_{i}\right)\right) \xi_{i j k 1}^{2}\left(\omega_{i}\right)+h\left(\xi_{i j k 2}\left(\omega_{i}\right)\right) \xi_{i j k 2}\left(\omega_{i}\right)\right) s_{k} .
\end{aligned}
$$

Portanto, a matriz $\boldsymbol{\Delta}_{i \gamma}$ fica dada por

$$
\begin{aligned}
& \Delta_{i \alpha}=\left.\frac{\partial^{2} L(\boldsymbol{\theta} \mid \boldsymbol{\omega})}{\partial \omega_{i} \partial \alpha}\right|_{\boldsymbol{\theta}=\hat{\boldsymbol{\theta}}, \boldsymbol{\omega}=\boldsymbol{\omega}_{0}}=b_{i 1}\left(\frac{\partial d_{i 1 \alpha}}{\partial \omega_{i}}-b_{i 1} c_{i 1} d_{i 1 \alpha}\right), \\
& \Delta_{i \beta_{r}}=\left.\frac{\partial^{2} L(\boldsymbol{\theta} \mid \boldsymbol{\omega})}{\partial \omega_{i} \partial \beta_{r}}\right|_{\boldsymbol{\theta}=\hat{\boldsymbol{\theta}}, \boldsymbol{\omega}=\boldsymbol{\omega}_{0}}=b_{i 1}\left(\frac{\partial d_{i 1 \beta_{r}}}{\partial \omega_{i}}-b_{i 1} c_{i 1} d_{i 1 \beta_{r}}\right), \quad r=1, \ldots, p, \\
& \Delta_{i \varsigma}=\left.\frac{\partial^{2} L(\boldsymbol{\theta} \mid \boldsymbol{\omega})}{\partial \omega_{i} \partial \varsigma}\right|_{\boldsymbol{\theta}=\hat{\boldsymbol{\theta}}, \boldsymbol{\omega}=\boldsymbol{\omega}_{0}}=b_{i 1}\left(\frac{\partial d_{i 1 \varsigma}}{\partial \omega_{i}}-b_{i 1} c_{i 1} d_{i 1 \varsigma}\right),
\end{aligned}
$$

em que $b_{i 1}=D_{\omega_{i 1}}^{-1}(\boldsymbol{\theta}), c_{i 1}=\frac{\partial D_{\omega_{i 1}}(\boldsymbol{\theta})}{\partial \omega_{i}}$ e $\boldsymbol{d}_{i 1 \gamma}=\frac{\partial D_{\omega_{i 1}}(\boldsymbol{\theta})}{\partial \boldsymbol{\gamma}}$

\section{Perturbação aditiva de uma variável explicativa}

Consideremos a perturbação dos valores da $p$-ésima variável explicativa, assumida contínua, como $x_{i j p}\left(\omega_{i}\right)=x_{i j p}+\omega_{i}$, em que $\omega_{i}$ representa o $i$-ésimo elemento do vetor $\boldsymbol{\omega}=\left[\omega_{1}, \ldots, \omega_{n}\right]^{\top}$ que denota o vetor de perturbações do modelo. Nesse caso, quando $\boldsymbol{\omega}=\boldsymbol{\omega}_{0}=[0, \ldots, 0]^{\top}$, o modelo perturbado se reduz ao modelo postulado, isto é, $L(\boldsymbol{\theta})=L(\boldsymbol{\theta} \mid \boldsymbol{\omega})$, em que

$$
\begin{aligned}
& L(\boldsymbol{\theta} \mid \boldsymbol{\omega})=\sum_{i=1}^{n} \log \left(D_{\omega_{i 2}}(\boldsymbol{\theta})\right), \quad D_{\omega_{i 2}}(\boldsymbol{\theta})=\sum_{k=1}^{q} \frac{v_{k}}{\sqrt{\pi}} A_{\omega_{i 2 k}}(\boldsymbol{\theta}) B_{\omega_{i 2 k}}(\boldsymbol{\theta}), \\
& A_{\omega_{i 2 k}(\boldsymbol{\theta})}=\exp \left(\sum_{j \in O}\left[-\frac{1}{2} \log (8 \pi)+\log \left(\xi_{i j k 1}\left(\omega_{i}\right)\right)-\frac{1}{2} \xi_{i j k 2}^{2}\left(\omega_{i}\right)\right]\right),
\end{aligned}
$$


$B_{\omega_{i 2 k}}(\boldsymbol{\theta})=\exp \left(\sum_{j \in C} \log \left(1-\Phi\left(\xi_{i j k 2}\left(\omega_{i}\right)\right)\right), \quad \xi_{i j k 2}\left(\omega_{i}\right)=\frac{2}{\alpha} \operatorname{senh}\left(\frac{y_{i j}-\boldsymbol{x}_{i j}^{\top}\left(\omega_{i}\right) \boldsymbol{\beta}-\sqrt{2 \varsigma} s_{k}}{2}\right)\right.$,

e

$$
\xi_{i j k 1}\left(\omega_{i}\right)=\frac{2}{\alpha} \cosh \left(\frac{y_{i j}-\boldsymbol{x}_{i j}^{\top}\left(\omega_{i}\right) \boldsymbol{\beta}-\sqrt{2 \varsigma} s_{k}}{2}\right)
$$

$\operatorname{com} \boldsymbol{x}_{i j}^{\top}\left(\omega_{i}\right) \boldsymbol{\beta}=\beta_{1} x_{i j 1}+\ldots+\beta_{p-1} x_{i j, p-1}+\beta_{p} x_{i j p}\left(\omega_{i}\right)$. A matriz $\boldsymbol{\Delta}_{i \gamma}$, com $\gamma=\left[\alpha, \beta_{r}, \varsigma\right]^{\top}$, pode ser escrita como

$$
\boldsymbol{\Delta}_{i \gamma}=\frac{\partial^{2} L(\boldsymbol{\theta} \mid \boldsymbol{\omega})}{\partial \omega_{i} \partial \boldsymbol{\gamma}}=\frac{\partial}{\partial \omega_{i}}\left(\frac{\partial L(\boldsymbol{\theta} / \boldsymbol{\omega})}{\partial \boldsymbol{\gamma}}\right)=\frac{\partial}{\partial \omega_{i}}\left(\sum_{i=1}^{n} D_{\omega_{i 2}}^{-1}(\boldsymbol{\theta}) \frac{\partial D_{\omega_{i 2}}(\boldsymbol{\theta})}{\partial \boldsymbol{\gamma}}\right)
$$

então

$$
\boldsymbol{\Delta}_{i \gamma}=D_{\omega_{i 2}}^{-1}(\boldsymbol{\theta}) \frac{\partial^{2} D_{\omega_{i 2}}(\boldsymbol{\theta})}{\partial \omega_{i} \partial \gamma}-D_{\omega_{i 2}}^{-2}(\boldsymbol{\theta}) \frac{\partial D_{\omega_{i 2}}(\boldsymbol{\theta})}{\partial \omega_{i}} \frac{\partial D_{\omega_{i 2}}(\boldsymbol{\theta})}{\partial \boldsymbol{\gamma}} .
$$

Se definimos $b_{i 2}=D_{\omega_{i 2}}^{-1}(\boldsymbol{\theta}), c_{i 2}=\frac{\partial D_{\omega_{i 2}}(\boldsymbol{\theta})}{\partial \omega_{i}}$ e $\boldsymbol{d}_{i 2 \gamma}=\frac{\partial D_{\omega_{i 2}}(\boldsymbol{\theta})}{\partial \boldsymbol{\gamma}}$, temos que

$$
\boldsymbol{\Delta}_{i \gamma}=b_{i 2}\left(\frac{\partial \boldsymbol{d}_{i 2 \gamma}}{\partial \omega_{i}}-b_{i 2} c_{i 2} \boldsymbol{d}_{i 2 \gamma}\right), \quad \boldsymbol{d}_{i 2 \gamma}=\sum_{k=1}^{q} \frac{v_{k}}{\sqrt{\pi}} A_{\omega_{i 2 k}}(\boldsymbol{\theta}) B_{\omega_{i 2 k}}(\boldsymbol{\theta}) \boldsymbol{F}_{\omega_{i 2 k \gamma}}(\boldsymbol{\theta}),
$$

com

$$
\boldsymbol{F}_{\omega_{i 2 k \gamma}}(\boldsymbol{\theta})=\sum_{j \in O}\left[\xi_{i j k 1}^{-1}\left(\omega_{i}\right) \frac{\partial \xi_{i j k 1}\left(\omega_{i}\right)}{\partial \gamma}-\xi_{i j k 2}\left(\omega_{i}\right) \frac{\partial \xi_{i j k 2}\left(\omega_{i}\right)}{\partial \gamma}\right]-\sum_{j \in C} h\left(\xi_{i j k 2}\left(\omega_{i}\right)\right) \frac{\partial \xi_{i j k 2}\left(\omega_{i}\right)}{\partial \gamma} .
$$

Em primeiro lugar vamos calcular $c_{i 2}$

$$
c_{i 2}=\sum_{k=1}^{q} \frac{v_{k}}{\sqrt{\pi}} A_{\omega_{i 2 k}}(\boldsymbol{\theta}) B_{\omega_{i 2 k}}(\boldsymbol{\theta}) F\left(x_{i j p}\left(\omega_{i}\right)\right)
$$

em que

$$
\begin{aligned}
F\left(x_{i j p}\left(\omega_{i}\right)\right) & =\sum_{j \in O}\left[\xi_{i j k 1}^{-1}\left(\omega_{i}\right) \frac{\partial \xi_{i j k 1}\left(\omega_{i}\right)}{\partial \omega_{i}}-\xi_{i j k 2}\left(\omega_{i}\right) \frac{\partial \xi_{i j k 2}\left(\omega_{i}\right)}{\partial \omega_{i}}\right]-\sum_{j \in C} h\left(\xi_{i j k 2}\left(\omega_{i}\right)\right) \frac{\partial \xi_{i j k 2}\left(\omega_{i}\right)}{\partial \omega_{i}} \\
& =-\frac{1}{2} \beta_{p} \sum_{j \in O}\left[\xi_{i j k 3}\left(\omega_{i}\right)-\xi_{i j k 2}\left(\omega_{i}\right) \xi_{i j k 1}\left(\omega_{i}\right)\right]+\frac{1}{2} \beta_{p} \sum_{j \in C} h\left(\xi_{i j k 2}\left(\omega_{i}\right)\right) \xi_{i j k 1}\left(\omega_{i}\right)
\end{aligned}
$$

e

$$
\xi_{i j k 3}\left(\omega_{i}\right)=\tanh \left(\frac{y_{i j}-\boldsymbol{x}_{i j}^{\top}\left(\omega_{i}\right) \boldsymbol{\beta}-\sqrt{2 \varsigma} s_{k}}{2}\right)
$$


Em segundo lugar,

$$
\frac{\partial \boldsymbol{d}_{i 2 \gamma}}{\partial \omega_{i}}=\sum_{k=1}^{q} \frac{v_{k}}{\sqrt{\pi}}\left[\frac{\partial\left(A_{\omega_{i 2 k}}(\boldsymbol{\theta}) B_{\omega_{i 2 k}}(\boldsymbol{\theta})\right)}{\partial \omega_{i}} \boldsymbol{F}_{\omega_{i 2 k \gamma}}(\boldsymbol{\theta})+A_{\omega_{i 2 k}}(\boldsymbol{\theta}) B_{\omega_{i 2 k}}(\boldsymbol{\theta}) \frac{\partial \boldsymbol{F}_{\omega_{i 2 k \gamma}}(\boldsymbol{\theta})}{\partial \omega_{i}}\right],
$$

com

$$
\begin{aligned}
\frac{\partial\left(A_{\omega_{i 2 k}}(\boldsymbol{\theta}) B_{\omega_{i 2 k}}(\boldsymbol{\theta})\right)}{\partial \omega_{i}} & =A_{\omega_{i 2 k}}(\boldsymbol{\theta}) B_{\omega_{i 2 k}}(\boldsymbol{\theta}) F\left(x_{i j p}\left(\omega_{i}\right)\right) \mathrm{e} \\
\frac{\partial \boldsymbol{F}_{\omega_{i 2 k \gamma}}(\boldsymbol{\theta})}{\partial \omega_{i}} & =\left[\frac{\partial F_{\omega_{i 2 k \alpha}}(\boldsymbol{\theta})}{\partial \omega_{i}}, \frac{\partial F_{\omega_{i 2 k \beta_{r}}}(\boldsymbol{\theta})}{\partial \omega_{i}}, \frac{\partial F_{\omega_{i 2 k \varsigma}}(\boldsymbol{\theta})}{\partial \omega_{i}}\right]^{\top}
\end{aligned}
$$

Portanto,

$$
\frac{\partial \boldsymbol{d}_{i 2 \gamma}}{\partial \omega_{i}}=\sum_{k=1}^{q} \frac{v_{k}}{\sqrt{\pi}} A_{\omega_{i 2 k}}(\boldsymbol{\theta}) B_{\omega_{i 2 k}}(\boldsymbol{\theta})\left[F\left(x_{i j p}\left(\omega_{i}\right)\right) \boldsymbol{F}_{\omega_{i 2 k \gamma}}(\boldsymbol{\theta})+\frac{\partial \boldsymbol{F}_{\omega_{i 2 k \gamma}}(\boldsymbol{\theta})}{\partial \omega_{i}}\right] .
$$

Derivando $\Delta_{i \alpha}$

Com base na equação (4.27) temos que

$$
\Delta_{i \alpha}=b_{i 2}\left(\frac{\partial d_{i 2 \alpha}}{\partial \omega_{i}}-b_{i 2} c_{i 2} d_{i 2 \alpha}\right),
$$

em que,

$$
d_{i 2 \alpha}=\sum_{k=1}^{q} \frac{v_{k}}{\sqrt{\pi}} A_{\omega_{i 2 k}}(\boldsymbol{\theta}) B_{\omega_{i 2 k}}(\boldsymbol{\theta}) F_{\omega_{i 2 k \alpha}}(\boldsymbol{\theta})
$$

com,

$$
F_{\omega_{i 2 k \alpha}}(\boldsymbol{\theta})=\frac{1}{\alpha} \sum_{j \in O}\left(\xi_{i j k 2}^{2}\left(\omega_{i}\right)-1\right)+\frac{1}{\alpha} \sum_{j \in C} h\left(\xi_{i j k 2}\left(\omega_{i}\right)\right) \xi_{i j k 2}\left(\omega_{i}\right) .
$$

Com base na equação (4.28), temos que

$$
\begin{aligned}
\frac{\partial d_{i 2 \alpha}}{\partial \omega_{i}}= & \sum_{k=1}^{q} \frac{v_{k}}{\sqrt{\pi}} A_{\omega_{i 2 k}}(\boldsymbol{\theta}) B_{\omega_{i 2 k}}(\boldsymbol{\theta})\left[F\left(x_{i j p}\left(\omega_{i}\right)\right) F_{\omega_{i 2 k \alpha}}(\boldsymbol{\theta})+\frac{\partial F_{\omega_{i 2 k \alpha}}(\boldsymbol{\theta})}{\partial \omega_{i}}\right] \\
\frac{\partial F_{\omega_{i 2 k \alpha}}(\boldsymbol{\theta})}{\partial \omega_{i}}= & -\frac{\beta_{p}}{2 \alpha}\left[\sum_{j \in O} 2 \xi_{i j k 2}\left(\omega_{i}\right) \xi_{i j k 1}\left(\omega_{i}\right)\right] \\
& -\frac{\beta_{p}}{2 \alpha}\left[\sum_{j \in C} \xi_{i j k 1}\left(\omega_{i}\right)\left(h\left(\xi_{i j k 2}\left(\omega_{i}\right)\right)+h^{\prime}\left(\xi_{i j k 2}\left(\omega_{i}\right)\right) \xi_{i j k 2}\left(\omega_{i}\right)\right)\right],
\end{aligned}
$$

em que

$$
h^{\prime}\left(\xi_{i j k 2}\left(\omega_{i}\right)\right)=\mathrm{d} h\left(\xi_{i j k 2}\left(\omega_{i}\right)\right) / \mathrm{d} \xi_{i j k 2}\left(\omega_{i}\right) .
$$


Derivando $\Delta_{i \beta_{r}}$

Com base na equação (4.27) temos que

$$
\Delta_{i \beta_{r}}=b_{i 2}\left(\frac{\partial d_{i 2 \beta_{r}}}{\partial \omega_{i}}-b_{i 2} c_{i 2} d_{i 2 \beta_{r}}\right),
$$

em que,

$$
d_{i 2 \beta_{r}}=\sum_{k=1}^{q} \frac{v_{k}}{\sqrt{\pi}} A_{\omega_{i 2 k}}(\boldsymbol{\theta}) B_{\omega_{i 2 k}}(\boldsymbol{\theta}) F_{\omega_{i 2 k \beta_{r}}}(\boldsymbol{\theta})
$$

com

$$
F_{\omega_{i 2 k \beta_{r}}}(\boldsymbol{\theta})=\frac{1}{2} \sum_{j \in O}\left(\xi_{i j k 1}\left(\omega_{i}\right) \xi_{i j k 2}\left(\omega_{i}\right)-\xi_{i j k 3}\left(\omega_{i}\right)\right) x_{i j r}+\frac{1}{2} \sum_{j \in C} h\left(\xi_{i j k 2}\left(\omega_{i}\right)\right) \xi_{i j k 1}\left(\omega_{i}\right) x_{i j r} .
$$

Com base na equação (4.28), temos que

$$
\frac{\partial d_{i 2 \beta_{r}}}{\partial \omega_{i}}=\sum_{k=1}^{q} \frac{v_{k}}{\sqrt{\pi}} A_{\omega_{i 2 k}}(\boldsymbol{\theta}) B_{\omega_{i 2 k}}(\boldsymbol{\theta})\left[F\left(x_{i j p}\left(\omega_{i}\right)\right) F_{\omega_{i 2 k \beta_{r}}}(\boldsymbol{\theta})+\frac{\partial F_{\omega_{i 2 k \beta_{r}}}(\boldsymbol{\theta})}{\partial \omega_{i}}\right]
$$

em que, se $r \neq p$

$$
\begin{aligned}
\frac{\partial F_{\omega_{i 2 k \beta_{r}}}(\boldsymbol{\theta})}{\partial \omega_{i}}= & -\frac{\beta_{p}}{4} \sum_{j \in O}\left(\xi_{i j k 1}^{2}\left(\omega_{i}\right)+\xi_{i j k 2}^{2}\left(\omega_{i}\right)-\left(1-\xi_{i j k 3}^{2}\left(\omega_{i}\right)\right)\right) x_{i j r} \\
& -\frac{\beta_{p}}{4} \sum_{j \in C}\left(h^{\prime}\left(\xi_{i j k 2}\left(\omega_{i}\right)\right) \xi_{i j k 1}^{2}\left(\omega_{i}\right)+h\left(\xi_{i j k 2}\left(\omega_{i}\right)\right) \xi_{i j k 2}\left(\omega_{i}\right)\right) x_{i j r} .
\end{aligned}
$$

e se $r=p$

$$
\begin{aligned}
\frac{\partial F_{\omega_{i 2 k \beta_{r}}}(\boldsymbol{\theta})}{\partial \omega_{i}}= & -\frac{\beta_{p}}{4} \sum_{j \in O}\left(\xi_{i j k 1}^{2}\left(\omega_{i}\right)+\xi_{i j k 2}^{2}\left(\omega_{i}\right)-\left(1-\xi_{i j k 3}^{2}\left(\omega_{i}\right)\right)\right) x_{i j r} \\
& +\frac{1}{2} \sum_{j \in O}\left(\xi_{i j k 3}\left(\omega_{i}\right)-\xi_{i j k 1}\left(\omega_{i}\right) \xi_{i j k 2}\left(\omega_{i}\right)\right) x_{i j r} \\
& -\frac{\beta_{p}}{4} \sum_{j \in C}\left(h^{\prime}\left(\xi_{i j k 2}\left(\omega_{i}\right)\right) \xi_{i j k 1}^{2}\left(\omega_{i}\right)+h\left(\xi_{i j k 2}\left(\omega_{i}\right)\right) \xi_{i j k 2}\left(\omega_{i}\right)\right) x_{i j r} \\
& -\frac{1}{2} \sum_{j \in C}\left(h\left(\xi_{i j k 2}\left(\omega_{i}\right)\right) \xi_{i j k 1}\left(\omega_{i}\right)\right) x_{i j r} .
\end{aligned}
$$

Derivando $\Delta_{i \varsigma}$

Com base na equação (4.27), temos que

$$
\Delta_{i \varsigma}=b_{i 2}\left(\frac{\partial d_{i 2 \varsigma}}{\partial \omega_{i}}-b_{i 2} c_{i 2} d_{i 2 \varsigma}\right),
$$


em que,

$$
d_{i 2 \varsigma}=\sum_{k=1}^{q} \frac{v_{k}}{\sqrt{\pi}} A_{\omega_{i 2 k}}(\boldsymbol{\theta}) B_{\omega_{i 2 k}}(\boldsymbol{\theta}) F_{\omega_{i 2 k \varsigma}}(\boldsymbol{\theta})
$$

com,

$$
F_{\omega_{i 2 k \varsigma}}(\boldsymbol{\theta})=\frac{1}{\sqrt{8 \varsigma}} \sum_{j \in O}\left(\xi_{i j k 1}\left(\omega_{i}\right) \xi_{i j k 2}\left(\omega_{i}\right)-\xi_{i j k 3}\left(\omega_{i}\right)\right) s_{k}+\frac{1}{\sqrt{8 \varsigma}} \sum_{j \in C} h\left(\xi_{i j k 2}\left(\omega_{i}\right)\right) \xi_{i j k 1}\left(\omega_{i}\right) s_{k} .
$$

Com base na equação (4.28), temos que

$$
\begin{aligned}
\frac{\partial d_{i 2 \varsigma}}{\partial \omega_{i}}= & \sum_{k=1}^{q} \frac{v_{k}}{\sqrt{\pi}} A_{\omega_{i 2 k}}(\boldsymbol{\theta}) B_{\omega_{i 2 k}}(\boldsymbol{\theta})\left[F\left(x_{i j p}\left(\omega_{i}\right)\right) F_{\omega_{i 2 k \varsigma}}(\boldsymbol{\theta})+\frac{\partial F_{\omega_{i 2 k \varsigma}}(\boldsymbol{\theta})}{\partial \omega_{i}}\right] \mathrm{e} \\
\frac{\partial F_{\omega_{i 2 k \varsigma}}(\boldsymbol{\theta})}{\partial \omega_{i}}= & -\frac{\beta_{p}}{\sqrt{32 \varsigma}} \sum_{j \in O}\left(\xi_{i j k 1}^{2}\left(\omega_{i}\right)+\xi_{i j k 2}^{2}\left(\omega_{i}\right)-\left(1-\xi_{i j k 3}^{2}\left(\omega_{i}\right)\right)\right) s_{k} \\
& -\frac{\beta_{p}}{\sqrt{32 \varsigma}} \sum_{j \in C}\left(h^{\prime}\left(\xi_{i j k 2}\left(\omega_{i}\right)\right) \xi_{i j k 1}^{2}\left(\omega_{i}\right)+h\left(\xi_{i j k 2}\left(\omega_{i}\right)\right) \xi_{i j k 2}\left(\omega_{i}\right)\right) s_{k} .
\end{aligned}
$$

Portanto, a matriz $\boldsymbol{\Delta}_{i \gamma}$ fica dada por

$$
\begin{aligned}
\Delta_{i \alpha} & =\left.\frac{\partial^{2} L(\boldsymbol{\theta} \mid \boldsymbol{\omega})}{\partial \omega_{i} \partial \alpha}\right|_{\boldsymbol{\theta}=\hat{\boldsymbol{\theta}}, \boldsymbol{\omega}=\boldsymbol{\omega}_{0}}=b_{i 2}\left(\frac{\partial d_{i 2 \alpha}}{\partial \omega_{i}}-b_{i 2} c_{i 2} d_{i 2 \alpha}\right) \\
\Delta_{i \beta_{r}} & =\left.\frac{\partial^{2} L(\boldsymbol{\theta} \mid \boldsymbol{\omega})}{\partial \omega_{i} \partial \beta_{r}}\right|_{\boldsymbol{\theta}=\hat{\boldsymbol{\theta}}, \boldsymbol{\omega}=\boldsymbol{\omega}_{0}}=b_{i 2}\left(\frac{\partial d_{i 2 \beta_{r}}}{\partial \omega_{i}}-b_{i 2} c_{i 2} d_{i 2 \beta_{r}}\right) \quad r=1, \ldots, p \quad \mathrm{e} \\
\Delta_{i \varsigma} & =\left.\frac{\partial^{2} L(\boldsymbol{\theta} \mid \boldsymbol{\omega})}{\partial \omega_{i} \partial \varsigma}\right|_{\boldsymbol{\theta}=\hat{\boldsymbol{\theta}}, \boldsymbol{\omega}_{=\boldsymbol{\omega}_{0}}=b_{i 2}\left(\frac{\partial d_{i 2 \varsigma}}{\partial \omega_{i}}-b_{i 2} c_{i 2} d_{i 2 \varsigma}\right)}
\end{aligned}
$$

em que $b_{i 2}=D_{\omega_{i 2}}^{-1}(\boldsymbol{\theta}), c_{i 2}=\frac{\partial D_{\omega_{i 2}}(\boldsymbol{\theta})}{\partial \omega_{i}}$ e $\boldsymbol{d}_{i 2 \gamma}=\frac{\partial D_{\omega_{i 2}}(\boldsymbol{\theta})}{\partial \gamma}$.

\subsubsection{Resíduos}

Uma extensão natural do resíduo tipo martingale definido na Seção 3.3.4 para os modelos logBirnbaum-Saunders mistos é a seguinte:

$$
r_{\mathrm{MT}_{i j}}=\operatorname{sign}\left(r_{\mathrm{M}_{i j}}\right) \sqrt{-2\left[r_{\mathrm{M}_{i j}}+\delta_{i j} \log \left(\delta_{i j}-r_{\mathrm{M}_{i j}}\right)\right]},
$$

em que $\delta_{i j}=0,1$ indicando se a $j$-ésima observação do $i$-ésimo grupo é censurada ou não, respectivamente, enquanto

$$
r_{\mathrm{M}_{i j}}=\delta_{i j}+\log \left(\Phi\left(-\frac{2}{\hat{\alpha}} \operatorname{senh}\left(\frac{y_{i j}-\hat{\mu}_{i j}}{2}\right)\right)\right)
$$

é o resíduo tipo martingale e $\hat{\mu}_{i j}=\boldsymbol{x}_{i j}^{\top} \hat{\boldsymbol{\beta}}+\tilde{b}_{i}$ com $\tilde{b}_{i}$ sendo a predição de $b_{i}$. Como $\hat{\mu}_{i j}$ envolve também $\tilde{b}_{i}$ denominamos $r_{\mathrm{MT}_{i j}}$ como resíduo tipo martingale condicional. O resíduo tipo martin- 
gale marginal é definido da mesma forma, contudo $\hat{\mu}_{i j}=\boldsymbol{x}_{i j}^{\top} \hat{\boldsymbol{\beta}}$. Não há estudos numéricos sobre a distribuição empírica dos resíduos tipo martingale definidos acima. Apresentamos a seguir um resumo de alguns estudos numéricos desenvolvidos para o resíduo tipo martingale condicional para modelos log-Birnbaum-Saunders de intercepto aleatório.

\subsubsection{Simulações}

Um pequeno estudo de simulação foi realizado para investigar o comportamento da distribuição empírica do resíduo tipo martingale condicional. Consideramos grupos de tamanhos $n=10,20,30$ cada um com $m=3$ repetições, ou seja, consideramos como número total de observações $N=$ 30, 60, 90. Os tempos de sobrevivência Birnbaum-Saunders foram gerados usando a expressão

$$
T_{i j}=\exp \left\{\beta_{1}+\beta_{2} x_{i j}+b_{i}\right\}\left[1+\frac{\alpha Z_{i j}^{2}}{2}+\alpha Z_{i j} \sqrt{1+\frac{\alpha^{2} Z_{i j}^{2}}{4}}\right],
$$

em que $b_{i} \sim \mathcal{N}(0,1.5), Z_{i j} \sim \mathcal{N}(0,1)$, com $i=1, \ldots, n$ e $j=1, \ldots, m$. Os valores para os coeficientes do modelo foram fixados em $\alpha=0,5 ; 1,0 ; 1,5, \beta_{1}=4, \beta_{2}=-2$ e a covariável foi gerada de uma variável aleatória distribuída $U(0,1)$. Os tempos de censura $C_{i j}$ foram gerados conforme variáveis independentes com distribuição uniforme definida no intervalo $(0, \tau)$, em que $\tau$ foi escolhido de maneira que as porcentagens de censura 10, 30 e 50 fossem atingidas. Assim, os tempos observados de sobrevivência foram $W_{i j}=\min \left(T_{i j}, C_{i j}\right)$ e o indicador de censura é $\delta_{i j}=0,1$ indica se a $j$-ésima observação do $i$-ésimo grupo é censurada ou não, respectivamente. Foram geradas 1000 amostras aleatórias para as diferentes configurações de $N=n m$, $\alpha$ e as diferentes porcentagens de censura. Para cada amostra o modelo log-BS de intercepto aleatório dado em (4.12) foi ajustado, em que $Y_{i j}^{*}=Y_{i j} \mid b_{i}=\log \left(W_{i j}\right) \operatorname{com} i=1, \ldots, n$ e $j=1, \ldots, m$. As Figuras (4.1)-(4.3) mostram os gráficos normais de probabilidades para os resíduos tipo martingale condicional. Baseando-se nas figuras em questão, podemos concluir que o resíduo tipo martingale condicional apresenta uma boa concordância com a distribuição normal padrão para os diferentes valores de $N, \alpha$ e as porcentagens de censura escolhidas. Em particular, quando a proporção de censura diminui, as distribuições empíricas dos resíduos tipo martingale condicional se aproximam mais rapidamente da distribuição normal padrão. 


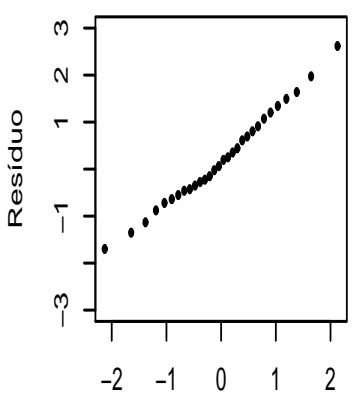

Percentis da N $(0,1)$ $\alpha=0,5 ; \mathrm{N}=30$

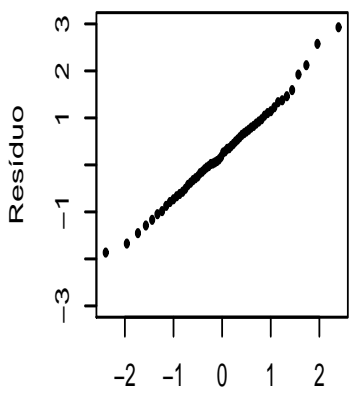

Percentis da N $(0,1)$ $\alpha=0,5: \mathrm{N}=60$

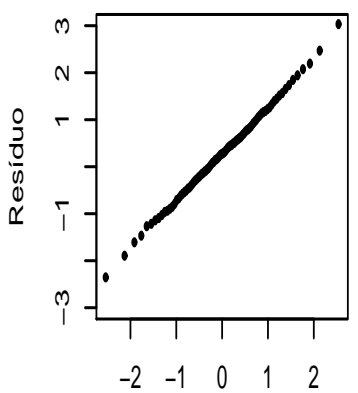

Percentis da N(0,1) $\alpha=0,5 ; \mathrm{N}=90$

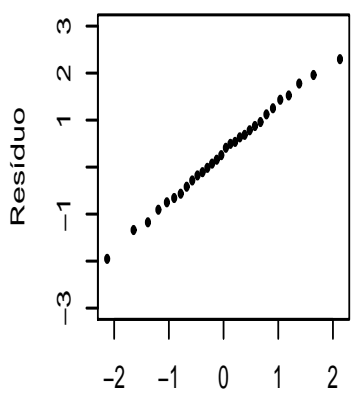

Percentis da $N(0,1)$ $\alpha=1,0 ; \mathrm{N}=30$

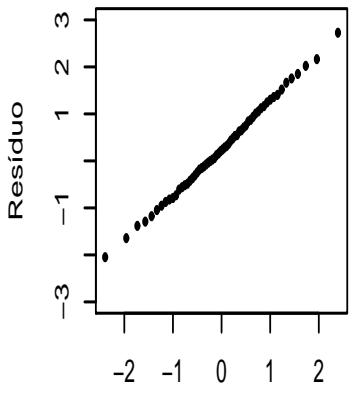

Percentis da N $(0,1)$
$\alpha=1,0 ; N=60$

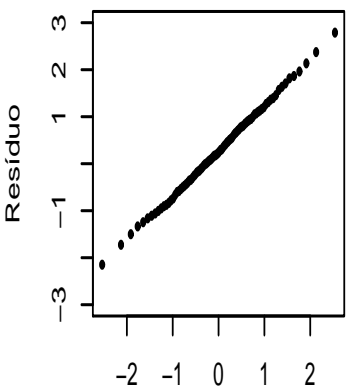

Percentis da N $(0,1)$ $\alpha=1,0 ; \mathrm{N}=90$

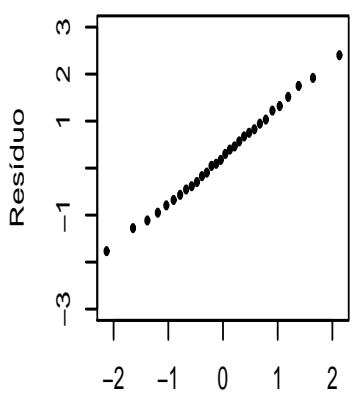

Percentis da N $(0,1)$ $\alpha=1,5 ; N=30$

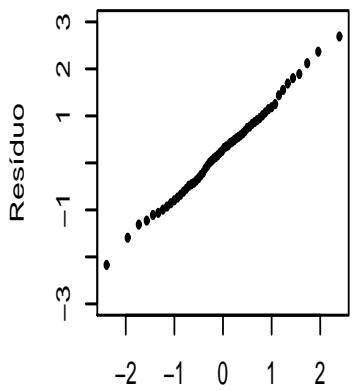

Percentis da N $(0,1)$ $\alpha=1,5 ; N=60$

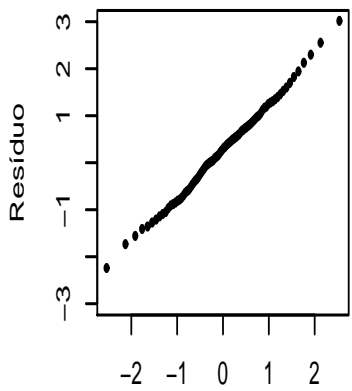

Percentis da N $(0,1)$

Figura 4.1: Gráficos normais de probabilidade para o resíduo tipo martingale condicional com porcentagem de censura de $10 \%$. 


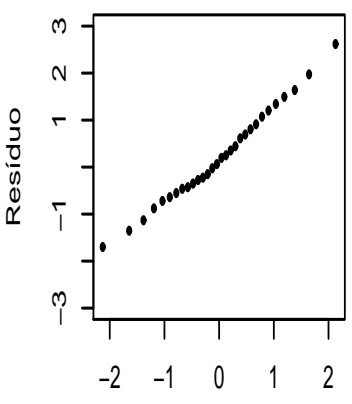

Percentis da $\mathrm{N}(0,1)$ $\alpha=0,5 ; N=30$

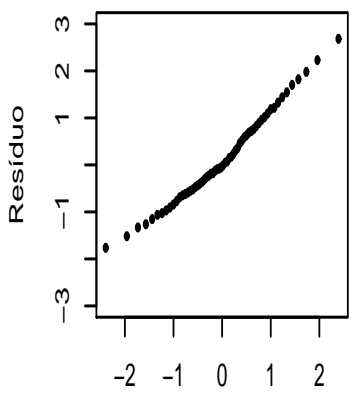

Percentis da N $(0,1)$ $\alpha=0,5 ; N=60$

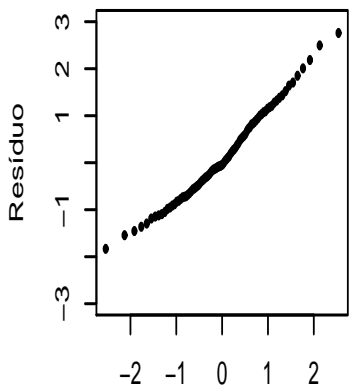

Percentis da N(0,1) $\alpha=0,5 ; \mathrm{N}=90$

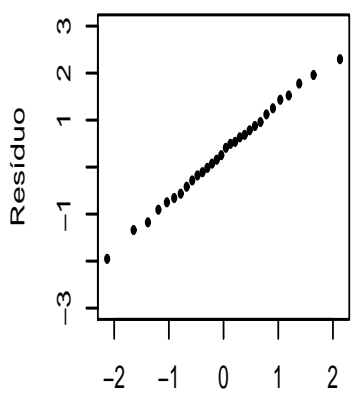

Percentis da N $(0,1)$ $\alpha=1,0 ; N=30$

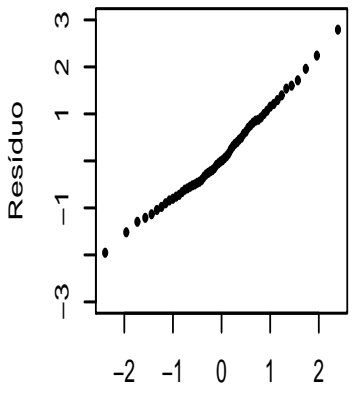

Percentis da N(0,1)
$\alpha=1,0 ; N=60$

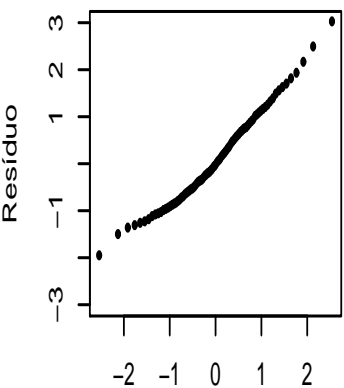

Percentis da N $(0,1)$ $\alpha=1,0 ; \mathrm{N}=90$

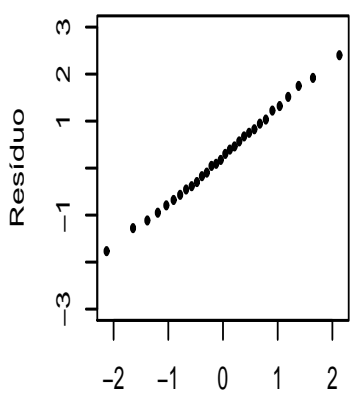

Percentis da N $(0,1)$ $\alpha=1,5 ; N=30$

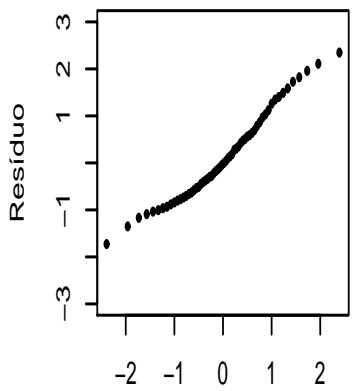

Percentis da N $(0,1)$ $\alpha=1,5 ; N=60$

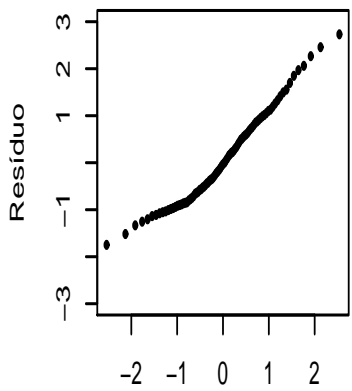

Percentis da N $(0,1)$ $\alpha=1,5 ; \mathrm{N}=90$

Figura 4.2: Gráficos normais de probabilidade para o resíduo tipo martingale condicional com porcentagem de censura de $30 \%$. 

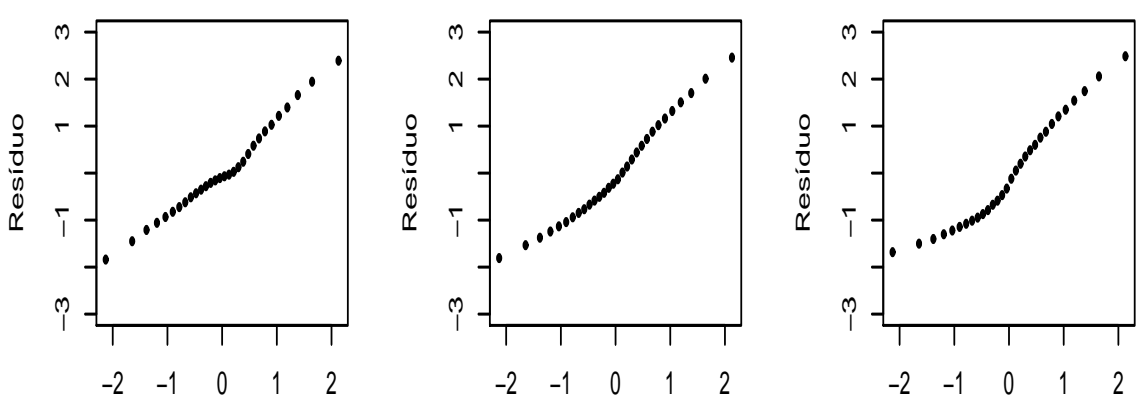

Percentis da N $(0,1)$ $\alpha=0,5 ; \mathrm{N}=30$

Percentis da N(0,1) $\alpha=1,0 ; N=30$

Percentis da N $(0,1)$ $\alpha=1,5 ; N=30$
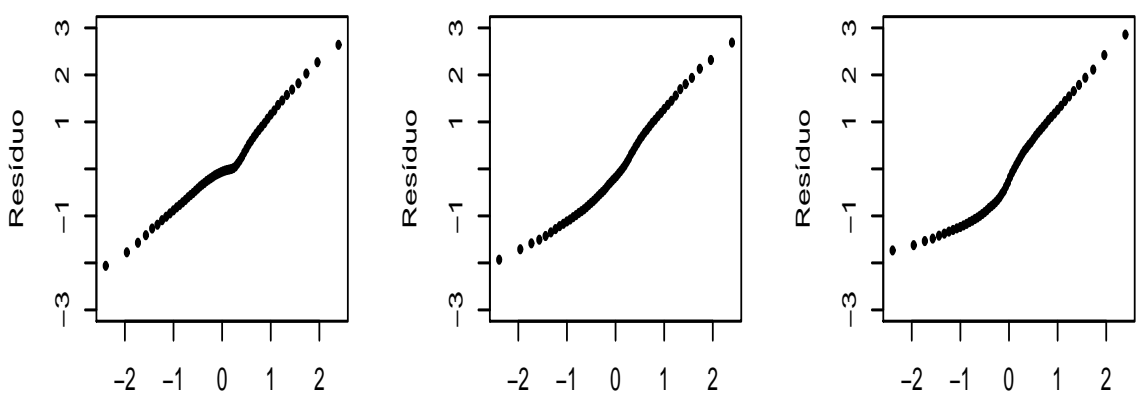

Percentis da N $(0,1)$ $\alpha=0,5 ; N=60$

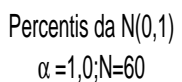

Percentis da N $(0,1)$ $\alpha=1,5 ; \mathrm{N}=60$
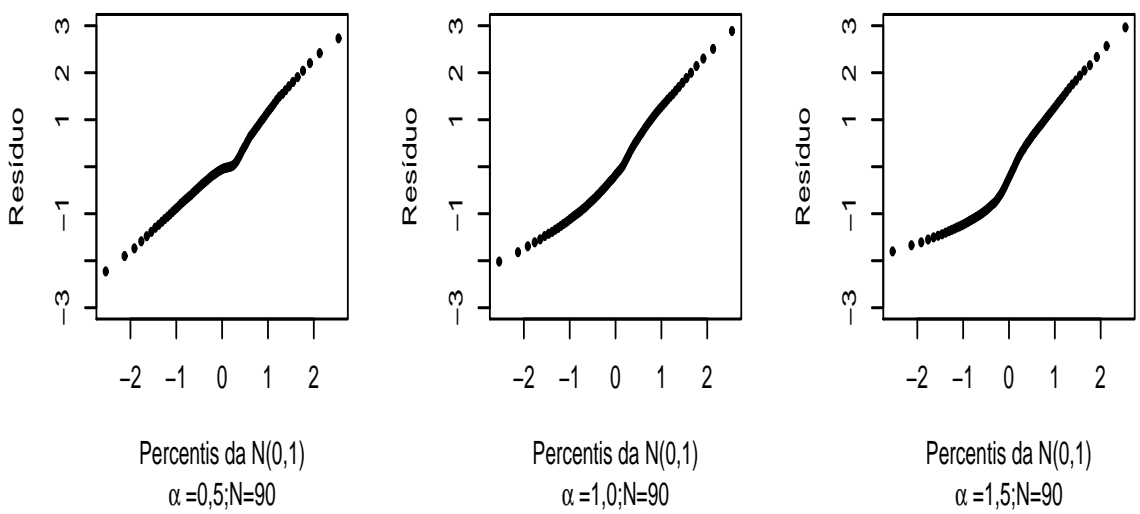

Percentis da N $(0,1)$

$$
\begin{gathered}
\text { Percentis da N }(0,1) \\
\alpha=1,5 ; \mathrm{N}=90
\end{gathered}
$$

Figura 4.3: Gráficos normais de probabilidade para o resíduo tipo martingale condicional com porcentagem de censura de $50 \%$. 


\subsection{Conclusões do capítulo}

Neste capítulo foram apresentados os modelos log-BS mistos para dados com censura e foi estudada a quadratura de Gauss-Hermite unidimensional e multidimensional. Especificamente, os modelos log-BS de intercepto aleatório foram estudados com base no enfoque marginal. Em particular e com base no trabalho de Tang et al. (2006), derivamos a função escore e a matriz hessiana. Uma discussão para testar o componente de variância em modelos lineares mistos foi feita e aplicou-se o teste da razão de verossimilhanças para os modelos mencionados anteriormente. A metodologia de influência local, proposta por Cook (1986) foi desenvolvida e um estudo de simulação para o resíduo tipo martingale condicional foi considerado para os modelos log-BS de intercepto aleatório.

Os modelos log-BS mistos para dados sem censura podem ser vistos como uma alternativa ao trabalho desenvolvido por Osorio (2006) que desenvolve os modelos elípticos com efeitos mistos sob o enfoque marginal (veja, por exemplo, Pinheiro e Bates, 2000; Verbeke e Molenberghs, 2000) e o enfoque hierárquico (veja, por exemplo, Pinheiro et al., 2001) usando a classe das misturas de escala normal (veja, por exemplo, Andrews e Mallows, 1974; Lange e Sinsheimer, 1993; Jamshidian, 1999).

A seguir estendemos os resultados desenvolvidos neste capítulo para os modelos log-BirnbaumSaunders-t (log-BS-t) mistos. 


\section{Capítulo 5}

\section{Modelos log-Birnbaum-Saunders-t mistos}

Os modelos log-Birnbaum-Saunders-t (log-BS-t) mistos surgem principalmente como uma alternativa para a obtenção de estimativas robustas, isto é, de acomodar observações aberrantes em relação aos modelos log-BS mistos e são motivados principalmente pelo trabalho de Barros et al. (2008), em que os autores argumentam que os modelos log-BS com efeitos fixos são sensíveis à observações atípicas, situação que se dá também entre os modelos lineares normal e os modelos lineares t-Student (veja, por exemplo, Lange et al., 1989).

Neste capítulo estendemos os cálculos apresentados no capítulo anterior para os modelos log-BSt mistos. Novamente, o procedimento Proc NLMIXED do SAS é sugerido para a obtenção das estimativas de máxima verossimilhança do modelo marginal e os respectivos erros padrão aproximados. Nos modelos log-BS-t com intercepto aleatório aplicamos o método de quadratura de Gauss-Hermite não adaptativo para aproximar o logaritmo da função de verossimilhança. Com essa aproximação derivamos a função escore e a matriz hessiana bem como as curvaturas normais de influência local para alguns esquemas de perturbação usuais. Discussões a respeito da predição dos resíduos tipo martingale são também apresentados. Finalmente, são discutidos alguns aspectos de robustez das estimativas de máxima verossimilhança. 


\subsection{Introdução}

Um importante ponto a ser considerado nos modelos log-BS-t mistos está relacionado à estimação dos graus de liberdade. Diferentes autores têm discutido esse tema, veja por exemplo, Lange et al. (1989), Fernandez e Steel (1999), Taylor e Verbyla (2004) e Leiva et al. (2008b). Esses autores descrevem a existência de dificuldades na estimação dos graus de liberdade, já que a função de verossimilhança apresenta máximos locais e não é limitada. Portanto neste trabalho, os graus de liberdade serão considerados fixos e serão escolhidos com base no critério de informação de Akaike (AIC). Na seguinte seção apresentamos os modelos log-BS-t mistos.

\subsection{Modelos log-Birnbaum-Saunders-t mistos}

Seja $Y_{i j}$ o $j$-ésimo logaritmo da resposta associada ao $i$-ésimo grupo, para $i=1, \ldots, n$ e $j=$ $1, \ldots, m_{i}$. Assumimos que o modelo log-Birnbaum-Saunders-t misto segue a seguinte estrutura hierárquica:

$$
\begin{aligned}
Y_{i j} \mid \boldsymbol{b}_{i} & \stackrel{\text { ind }}{\sim} \log \text {-BS-t }\left(\alpha, \boldsymbol{x}_{i j}^{\top} \boldsymbol{\beta}+\boldsymbol{z}_{i j}^{\top} \boldsymbol{b}_{i} ; \nu\right), \\
\boldsymbol{b}_{i} & \stackrel{\text { ind }}{\sim} \mathcal{N}_{q}(\mathbf{0}, \boldsymbol{D}),
\end{aligned}
$$

em que $\boldsymbol{x}_{i j}=\left[x_{i j 1}, \ldots, x_{i j p}\right]^{\top}$ contém valores de variáveis explicativas, $\boldsymbol{z}_{i j}=\left[z_{i j 1}, \ldots, z_{i j q}\right]^{\top}$ contém valores de variáveis explicativas associadas aos efeitos aleatórios, $\boldsymbol{\beta}=\left[\beta_{1}, \ldots, \beta_{p}\right]^{\top}$ é um vetor de efeitos fixos (parâmetros desconhecidos), $\boldsymbol{b}_{i}=\left[b_{i 1}, \ldots, b_{i q}\right]^{\top}$ é um vetor de efeitos aleatórios, 0 é um vetor de zeros e $\boldsymbol{D}=\boldsymbol{D}(\varsigma) \in \mathbb{R}^{q}$ é a matriz que contém os componentes de variância do modelo cujos elementos são $\varsigma=\left[\varsigma_{11} \ldots \varsigma_{q q}\right]^{\top}$. Seja $\boldsymbol{\theta}=\left[\alpha, \boldsymbol{\beta}^{\top}, \varsigma^{\top}\right]^{\top}$ o vetor de parâmetros a ser estimados no modelo acima. Analogamente à Seção 4.2, temos que o logaritmo da função de verossimilhança pode ser expresso na forma

$$
L(\boldsymbol{\theta})=\sum_{i=1}^{n} \log \left(\int_{\mathbb{R}^{q}}\left\{\prod_{j \in O} f_{i j}\left(y_{i j} \mid \mathbf{b}_{i} ; \alpha, \boldsymbol{\beta}\right) \prod_{j \in C} \Phi_{t}\left(-\kappa_{i j 2}\right)\right\} f\left(\boldsymbol{b}_{i} ; \boldsymbol{\varsigma}\right) \mathrm{d} \boldsymbol{b}_{i}\right),
$$

em que $O$ e $C$ representam os conjuntos de logaritmos dos tempos de falha ou censura, respectivamente, $\Phi_{t}(\cdot)$ é a função de distribuição acumulada de uma variável aleatória com distribuição t-Student padrão com $\nu$ graus de liberdade e

$$
f_{i j}\left(y_{i j} \mid \boldsymbol{b}_{i} ; \alpha, \boldsymbol{\beta}\right) \propto \exp \left(-\frac{\nu+1}{2} \log \left(1+\frac{\kappa_{i j 2}^{2}}{\nu}\right)+\log \left(\kappa_{i j 1}\right)\right),
$$

em que $\kappa_{i j 1}$ e $\kappa_{i j 2}$ foram definidos na equação (4.10).

Observação 8. Um caso particular que pode ser recuperado da equação (5.1), considerando dados com censura aleatória e $m_{i}=1$, para todo $i$, é o modelo proposto por Leiva et al. (2008a).

Na seguinte seção introduzimos o modelo log-Birnbaum-Saunders-t de intercepto aleatório para dados com censura aleatória, que será usado no Capítulo 6. 


\subsection{Modelos log-Birnbaum-Saunders-t de intercepto aleatório}

Seja $Y_{i j}$ a $j$-ésima medida no $i$-ésimo indivíduo, para dados com censura, definimos o modelo log-BS-t de intercepto aleatório por meio da seguinte estrutura hierárquica:

$$
\begin{aligned}
Y_{i j} \mid b_{i} & \stackrel{\text { ind }}{\sim} \log -\mathrm{BS}-\mathrm{t}\left(\alpha, \boldsymbol{x}_{i j}^{\top} \boldsymbol{\beta}+b_{i} ; \nu\right) \quad \mathrm{e} \\
b_{i} & \stackrel{\text { ind }}{\sim} \mathcal{N}(0, \varsigma), \quad i=1, \ldots, n \quad j=1, \ldots, m_{i},
\end{aligned}
$$

em que $\boldsymbol{x}_{i j}=\left[x_{i j 1}, \ldots, x_{i j p}\right]^{\top}$ contém valores das variáveis explicativas, $\boldsymbol{\beta}=\left[\beta_{1}, \ldots, \beta_{p}\right]^{\top}$ contém os parâmetros fixos do modelo e $b_{i}$ é o efeito aleatório associado ao $i$-ésimo grupo. Seja $\boldsymbol{\theta}=$ $\left[\alpha, \boldsymbol{\beta}^{\top}, \boldsymbol{\varsigma}^{\top}\right]^{\top}$ o vetor de parâmetros a ser estimados no modelo 5.4. Baseado na seção anterior, podemos escrever o logaritmo da função de verossimilhança expresso na forma

$$
L(\boldsymbol{\theta})=\sum_{i=1}^{n} \log \left(\int_{-\infty}^{+\infty}\left\{\prod_{j \in O} f_{i j}\left(y_{i j} \mid b_{i} ; \alpha, \boldsymbol{\beta}\right) \prod_{j \in C}\left[1-\Phi_{t}\left(\kappa_{i j 2}\right)\right]\right\} f\left(b_{i} ; \varsigma\right) \mathrm{d} b_{i}\right) .
$$

\subsubsection{Modelo marginal}

Com base na estrutura hierárquica do modelo (5.4) e na quadratura de Gauss-Hermite não adaptativa temos que,

$$
\begin{aligned}
L(\boldsymbol{\theta}) & \approx \sum_{i=1}^{n} \log \left(\sum_{k=1}^{q} \frac{v_{k}}{\sqrt{\pi}} A_{i k}^{\star}(\boldsymbol{\theta}) B_{i k}^{\star}(\boldsymbol{\theta})\right), \operatorname{com} \\
A_{i k}^{\star}(\boldsymbol{\theta}) & =\exp \left(\sum_{j \in O}\left[-\frac{\nu+1}{2} \log \left(1+\frac{\xi_{i j k 2}^{2}}{\nu}\right)+\log \left(\xi_{i j k 1}\right)\right]\right), \mathrm{e} \\
B_{i k}^{\star}(\boldsymbol{\theta}) & =\exp \left(\sum_{j \in C} \log \left(1-\Phi_{t}\left(\xi_{i j k 2}\right)\right)\right),
\end{aligned}
$$

em que $\xi_{i j k 1}$ e $\xi_{i j k 2}$ foram definidas na equação (4.18).

\subsubsection{Função escore}

Os seguintes cálculos da função escore são baseados na Seção 4.3.3.

$$
\boldsymbol{U}(\boldsymbol{\theta})=\frac{\partial L(\boldsymbol{\theta})}{\partial \boldsymbol{\theta}}=\sum_{i=1}^{n} \frac{\partial \log \left(D_{i}^{\star}(\boldsymbol{\theta})\right)}{\partial \boldsymbol{\theta}}=\sum_{i=1}^{n} \frac{1}{D_{i}^{\star}(\boldsymbol{\theta})} \frac{\partial D_{i}^{\star}(\boldsymbol{\theta})}{\partial \boldsymbol{\theta}},
$$

em que,

$$
D_{i}^{\star}(\boldsymbol{\theta})=\sum_{k=1}^{q} \frac{v_{k}}{\sqrt{\pi}} A_{i k}^{\star}(\boldsymbol{\theta}) B_{i k}^{\star}(\boldsymbol{\theta}) \quad \text { e } \quad \frac{\partial D_{i}^{\star}(\boldsymbol{\theta})}{\partial \boldsymbol{\theta}}=\sum_{k=1}^{q} \frac{v_{k}}{\sqrt{\pi}} A_{i k}^{\star}(\boldsymbol{\theta}) B_{i k}^{\star}(\boldsymbol{\theta}) F_{i k}^{\star}(\boldsymbol{\theta}),
$$


com

$$
\begin{aligned}
& F_{i k}^{\star}(\boldsymbol{\theta})=\sum_{j \in O}\left[\xi_{i j k 1}^{-1} \frac{\partial \xi_{i j k 1}}{\partial \boldsymbol{\theta}}-w\left(\xi_{i j k 2}^{2}\right) \xi_{i j k 2} \frac{\partial \xi_{i j k 2}}{\partial \boldsymbol{\theta}}\right]-\sum_{j \in C} h_{t}\left(\xi_{i j k 2}\right) \frac{\partial \xi_{i j k 2}}{\partial \boldsymbol{\theta}} \\
& w\left(\xi_{i j k 2}^{2}\right)=\frac{\nu+1}{\nu+\xi_{i j k 2}^{2}} \text { e } \quad h_{t}\left(\xi_{i j k 2}\right)=\frac{\phi_{t}\left(\xi_{i j k 2}\right)}{1-\Phi_{t}\left(\xi_{i j k 2}\right)},
\end{aligned}
$$

em que $\phi_{t}(\cdot)$ é a função de distribuição acumulada da distribuição t-Student padrão com $\nu$ graus de liberdade. Logo, com base na equação (5.7) temos os seguintes resultados

$$
\begin{aligned}
F_{i k \alpha}^{\star}(\boldsymbol{\theta}) & =\frac{1}{\alpha} \sum_{j \in O}\left(w\left(\xi_{i j k 2}^{2}\right) \xi_{i j k 2}^{2}-1\right)+\frac{1}{\alpha} \sum_{j \in C} h_{t}\left(\xi_{i j k 2}\right) \xi_{i j k 2} \\
F_{i k \beta_{r}}^{\star}(\boldsymbol{\theta}) & =\frac{1}{2} \sum_{j \in O}\left(w\left(\xi_{i j k 2}^{2}\right) \xi_{i j k 1} \xi_{i j k 2}-\xi_{i j k 3}\right) x_{i j r}+\frac{1}{2} \sum_{j \in C} h_{t}\left(\xi_{i j k 2}\right) \xi_{i j k 1} x_{i j r} \quad \mathrm{e} \\
F_{i k \varsigma}^{\star}(\boldsymbol{\theta}) & =\frac{1}{\sqrt{8 \varsigma}} \sum_{j \in O}\left(w\left(\xi_{i j k 2}^{2}\right) \xi_{i j k 1} \xi_{i j k 2}-\xi_{i j k 3}\right) s_{k}+\frac{1}{\sqrt{8 \varsigma}} \sum_{j \in C} h_{t}\left(\xi_{i j k 2}\right) \xi_{i j k 1} s_{k} .
\end{aligned}
$$

Matricialmente $\boldsymbol{U}(\boldsymbol{\theta})$ pode ser expresso na forma

$$
\boldsymbol{U}(\boldsymbol{\theta})=\left[U_{\alpha}(\boldsymbol{\theta}) U_{\beta_{1}}(\boldsymbol{\theta}), \ldots, U_{\beta_{p}}(\boldsymbol{\theta}) U_{\varsigma}(\boldsymbol{\theta})\right]^{\top},
$$

em que, aproximadamente temos

$$
\begin{aligned}
U_{\alpha}(\boldsymbol{\theta}) & =\mathbf{1}^{\top} \boldsymbol{G}_{1}^{\star} \boldsymbol{G}_{2}^{\star} \mathbf{1}, \\
U_{\beta_{r}}(\boldsymbol{\theta}) & =\mathbf{1}^{\top} \boldsymbol{G}_{1}^{\star} \boldsymbol{G}_{3 r}^{\star} \mathbf{1}, \quad r=1, \ldots, p, \quad \mathrm{e} \\
U_{\varsigma}(\boldsymbol{\theta}) & =\mathbf{1}^{\top} \boldsymbol{G}_{1}^{\star} \boldsymbol{G}_{4}^{\star} \mathbf{1},
\end{aligned}
$$

com $1 \in \mathbb{R}^{n}$ sendo um vetor de dimensão $n$ com 1 em todas as posições,

$$
\begin{aligned}
\boldsymbol{G}_{1}^{\star} & =\operatorname{diag}\left\{1 / D_{1}^{\star}(\boldsymbol{\theta}), \ldots, 1 / D_{n}^{\star}(\boldsymbol{\theta})\right\}, \\
\boldsymbol{G}_{2}^{\star} & =\operatorname{diag}\left\{\partial D_{1}^{\star}(\boldsymbol{\theta}) / \partial \alpha, \ldots, \partial D_{n}^{\star}(\boldsymbol{\theta}) / \partial \alpha\right\}, \\
\boldsymbol{G}_{3 r}^{\star} & =\operatorname{diag}\left\{\partial D_{1}^{\star}(\boldsymbol{\theta}) / \partial \beta_{r}, \ldots, \partial D_{n}^{\star}(\boldsymbol{\theta}) / \partial \beta_{r}\right\}, \quad r=1, \ldots, p, \quad \mathrm{e} \\
\boldsymbol{G}_{4}^{\star} & =\operatorname{diag}\left\{\partial D_{1}^{\star}(\boldsymbol{\theta}) / \partial \varsigma, \ldots, \partial D_{n}^{\star}(\boldsymbol{\theta}) / \partial \varsigma,\right\} .
\end{aligned}
$$

\subsubsection{Robustez das estimativas}

Observando as funções escore $U_{\alpha}(\boldsymbol{\theta}), U_{\beta_{r}}(\boldsymbol{\theta})$ e $U_{\varsigma}(\boldsymbol{\theta})$ considerando que não há dados censurados notamos que a quantidade

$$
w\left(\xi_{i j k 2}^{2}\right)=\frac{\nu+1}{\nu+\xi_{i j k 2}^{2}}
$$


aparece nessas expressões. Note que $w\left(\xi_{i j k 2}^{2}\right) \rightarrow 1$ quando $\nu \rightarrow \infty$. Além disso, $w\left(\xi_{i j k 2}^{2}\right)$ é inversamente proporcional à quantidade

$$
\xi_{i j k 2}^{2}=\left[\frac{2}{\alpha} \operatorname{senh}\left(C_{i j k}\right)\right]^{2}
$$

em que $C_{i j k}=\left(y_{i j}-\boldsymbol{x}_{i j}^{\top} \boldsymbol{\beta}-\sqrt{2 \varsigma} s_{k}\right) / 2$. Assim, há indícios de robustez das estimativas de máxima verossimilhança $\widehat{\alpha}, \widehat{\beta}$ e $\widehat{\varsigma}$ contra observações aberrantes no sentido do resíduo $\widehat{\xi}_{i j k 2}=\frac{2}{\widehat{\alpha}} \operatorname{senh}\left(\widehat{C}_{i j k}\right)$ com $\widehat{C}_{i j k}=\left(y_{i j}-\boldsymbol{x}_{i j}^{\top} \widehat{\boldsymbol{\beta}}-\sqrt{2 \widehat{\varsigma}} s_{k}\right) / 2$, similarmente ao que foi observado por Barros et al. (2008) para o caso de modelos log-BS-t de efeitos fixos.

\subsubsection{Matriz hessiana}

Para obter a matriz hessiana, baseado na Seção 4.3.4, devemos calcular

$$
\ddot{\boldsymbol{L}}_{\theta \theta}=\sum_{i=1}^{n}\left\{\frac{1}{D_{i}^{\star}(\boldsymbol{\theta})} \frac{\partial^{2} D_{i}^{\star}(\boldsymbol{\theta})}{\partial \boldsymbol{\theta} \partial \boldsymbol{\theta}^{\top}}-\frac{1}{D_{i}^{\star}(\boldsymbol{\theta})} \frac{\partial D_{i}^{\star}(\boldsymbol{\theta})}{\partial \boldsymbol{\theta}} \frac{\partial D_{i}^{\star}(\boldsymbol{\theta})}{\partial \boldsymbol{\theta}^{\top}}\right\} .
$$

As matrices associadas com a derivada $\frac{\partial^{2} D_{i}^{\star}(\boldsymbol{\theta})}{\partial \boldsymbol{\theta} \partial \boldsymbol{\theta}^{\top}}$ da equação (5.8), seguindo a Seção 4.3 .4 são $\frac{\partial F_{i k \alpha}^{\star}(\boldsymbol{\theta})}{\partial \alpha}, \frac{\partial F_{i k \beta_{r}}^{\star}(\boldsymbol{\theta})}{\partial \alpha}, \frac{\partial F_{i k \varsigma}^{\star}(\boldsymbol{\theta})}{\partial \alpha}, \frac{\partial F_{i k \beta_{r}}^{\star}(\boldsymbol{\theta})}{\partial \beta_{s}}, \frac{\partial F_{i k \beta_{r}}^{\star}(\boldsymbol{\theta})}{\partial \varsigma} \mathrm{e} \frac{\partial F_{i k \varsigma}^{\star}(\boldsymbol{\theta})}{\partial \varsigma}$, as quais detalhamos a seguir.

$$
\begin{aligned}
\frac{\partial F_{i k \alpha}^{\star}(\boldsymbol{\theta})}{\partial \alpha}= & \frac{1}{\alpha^{2}} \sum_{j \in O}\left[1-3 \xi_{i j k 2}^{2} w\left(\xi_{i j k 2}^{2}\right)-\xi_{i j k 1} \xi_{i j k 2}^{2} w^{\prime}\left(\xi_{i j k 2}^{2}\right)\right] \\
& -\frac{1}{\alpha^{2}} \sum_{j \in C}\left(h_{t}^{\prime}\left(\xi_{i j k 2}\right) \xi_{i j k 2}^{2}+2 h_{t}\left(\xi_{i j k 2}\right) \xi_{i j k 2}\right), \\
\frac{\partial F_{i k \beta_{r}}^{\star}(\boldsymbol{\theta})}{\partial \alpha}= & -\frac{2}{\alpha^{3}}\left(\sum_{j \in O} \operatorname{senh}\left(2 C_{i j k}\right)\left[w^{\prime}\left(\xi_{i j k 2}^{2}\right) \xi_{i j k 2}^{2}+w\left(\xi_{i j k 2}^{2}\right)\right] x_{i j r}\right) \\
& -\frac{1}{2 \alpha}\left(\sum_{j \in C}\left(\xi_{i j k 1} h_{t}\left(\xi_{i j k 2}\right)+\xi_{i j k 1} \xi_{i j k 2} h_{t}^{\prime}\left(\xi_{i j k 2}\right)\right) x_{i j r}\right), \\
\frac{\partial F_{i k \varsigma}^{\star}(\boldsymbol{\theta})}{\partial \alpha}= & -\frac{s_{k}}{\sqrt{2 \alpha^{2} \varsigma}}\left(\sum_{j \in O} \xi_{i j k 1} \xi_{i j k 2}\left[w^{\prime}\left(\xi_{i j k 2}^{2}\right) \xi_{i j k 2}^{2}+w\left(\xi_{i j k 2}^{2}\right)\right]\right) s_{k} \\
& -\frac{1}{\sqrt{8 \alpha^{2} \varsigma}}\left(\sum_{j \in C}\left(\xi_{i j k 1} h_{t}\left(\xi_{i j k 2}\right)+\xi_{i j k 1} \xi_{i j k 2} h_{t}^{\prime}\left(\xi_{i j k 2}\right)\right)\right) s_{k},
\end{aligned}
$$




$$
\begin{aligned}
\frac{\partial F_{i k \beta_{s}}^{\star}(\boldsymbol{\theta})}{\partial \beta_{r}}= & -\frac{1}{4} \sum_{j \in O}\left[w\left(\xi_{i j k 2}^{2}\right)\left(\xi_{i j k 1}^{2}+\xi_{i j k 2}^{2}\right)+w^{\prime}\left(\xi_{i j k 2}^{2}\right) \xi_{i j k 1} \xi_{i j k 2}^{2}-\operatorname{sech}^{2}\left(C_{i j k}\right)\right] x_{i j r} x_{i j s} \\
& -\frac{1}{4} \sum_{j \in C}\left[\xi_{i j k 1}^{2} h_{t}^{\prime}\left(\xi_{i j k 2}\right)+\xi_{i j k 2} h_{t}\left(\xi_{i j k 2}\right)\right] x_{i j r} x_{i j s}, \\
\frac{\partial F_{i k \beta_{r}}^{\star}(\boldsymbol{\theta})}{\partial \varsigma}= & -\frac{1}{\sqrt{32 \varsigma}} \sum_{j \in O}\left[w\left(\xi_{i j k 2}^{2}\right)\left(\xi_{i j k 1}^{2}+\xi_{i j k 2}^{2}\right)+2 w^{\prime}\left(\xi_{i j k 2}^{2}\right) \xi_{i j k 1}^{2} \xi_{i j k 2}^{2}-\operatorname{sech}^{2}\left(C_{i j k}\right)\right] x_{i j r} s_{k} \\
& -\frac{1}{\sqrt{32 \varsigma}} \sum_{j \in C}\left(\xi_{i j k 1}^{2} h_{t}^{\prime}\left(\xi_{i j k 2}\right)+\xi_{i j k 2} h_{t}\left(\xi_{i j k 2}\right)\right) x_{i j r} s_{k}, \\
\frac{\partial F_{i k \varsigma}^{\star}(\boldsymbol{\theta})}{\partial \varsigma}= & -\frac{1}{\sqrt{32 \varsigma^{3}}} \sum_{j \in O}\left(w\left(\xi_{i j k 2}^{2}\right) \xi_{i j k 1} \xi_{i j k 2}-\xi_{i j k 3}\right) s_{k} \\
& -\frac{1}{\sqrt{64 \varsigma^{2}}} \sum_{j \in O}\left[2 w^{\prime}\left(\xi_{i j k 2}\right) \xi_{i j k 1}^{2} \xi_{i j k 2}^{2}+w\left(\xi_{i j k 2}^{2}\right)\left(\xi_{i j k 1}^{2}+\xi_{i j k 2}^{2}\right)-\operatorname{sech}^{2}\left(C_{i j k}\right)\right] s_{k}^{2} \\
& -\frac{s_{k}}{\sqrt{32 \varsigma^{3}}} \sum_{j \in C} h_{t}\left(\xi_{i j k 2}\right) \xi_{i j k 1}-\frac{1}{\sqrt{64 \varsigma^{2}}} \sum_{j \in C}\left(\left(\xi_{i j k 1}^{2} h_{t}^{\prime}\left(\xi_{i j k 2}\right)+\xi_{i j k 2} h_{t}\left(\xi_{i j k 2}\right)\right)\right) s_{k}^{2},
\end{aligned}
$$

em que

$$
\begin{aligned}
h_{t}^{\prime}\left(\xi_{i j k 2}\right) & =\frac{\mathrm{d} h_{t}\left(\xi_{i j k 2}\right)}{\mathrm{d} \xi_{i j k 2}}=h_{t}^{2}\left(\xi_{i j k 2}\right)-\xi_{i j k 2} w\left(\xi_{i j k 2}^{2}\right) h_{t}\left(\xi_{i j k 2}\right) \quad \mathrm{e} \\
\xi_{i j k 3} & =\tanh \left(\frac{y_{i j}-\boldsymbol{x}_{i}^{\top} \boldsymbol{\beta}}{2}\right) .
\end{aligned}
$$

Matricialmente, $\ddot{\boldsymbol{L}}_{\theta \theta}$ pode ser escrita como

$$
\ddot{\boldsymbol{L}}_{\theta \theta}=\left[\begin{array}{ccc}
\ddot{L}_{\alpha \alpha} & \ddot{\boldsymbol{L}}_{\alpha \beta} & \ddot{L}_{\alpha \varsigma} \\
\ddot{\boldsymbol{L}}_{\beta \alpha} & \ddot{\boldsymbol{L}}_{\beta \beta} & \ddot{\boldsymbol{L}}_{\beta \varsigma} \\
\ddot{L}_{\varsigma \alpha} & \ddot{\boldsymbol{L}}_{\varsigma \beta} & \ddot{L}_{\varsigma \varsigma}
\end{array}\right],
$$

em que

$$
\begin{aligned}
\ddot{L}_{\alpha \alpha}=\mathbf{1}^{\top}\left(\boldsymbol{G}_{1}^{\star} \boldsymbol{G}_{5}^{\star}-\left(\boldsymbol{G}_{1}^{\star}\right)^{2}\left(\boldsymbol{G}_{2}^{\star}\right)^{2}\right) \mathbf{1}, & \ddot{L}_{\alpha \beta_{r}}=\mathbf{1}^{\top}\left(\boldsymbol{G}_{1}^{\star} \boldsymbol{G}_{6 r}^{\star}-\left(\boldsymbol{G}_{1}^{\star}\right)^{2} \boldsymbol{G}_{2}^{\star} \boldsymbol{G}_{3}^{\star}\right) \mathbf{1}, \\
\ddot{L}_{\alpha \varsigma}=\mathbf{1}^{\top}\left(\boldsymbol{G}_{1}^{\star} \boldsymbol{G}_{7}^{\star}-\left(\boldsymbol{G}_{1}^{\star}\right)^{2} \boldsymbol{G}_{2}^{\star} \boldsymbol{G}_{4}^{\star}\right) \mathbf{1}, & \ddot{L}_{\beta_{r} \beta_{s}}=\mathbf{1}^{\top}\left(\boldsymbol{G}_{1}^{\star} \boldsymbol{G}_{8 r s}^{\star}-\left(\boldsymbol{G}_{1}^{\star}\right)^{2}\left(\boldsymbol{G}_{3}^{\star}\right)^{2}\right) \mathbf{1}, \\
\ddot{L}_{\beta_{r} \varsigma}=\mathbf{1}^{\top}\left(\boldsymbol{G}_{1}^{\star} \boldsymbol{G}_{9 r}^{\star}-\left(\boldsymbol{G}_{1}^{\star}\right)^{2} \boldsymbol{G}_{3}^{\star} \boldsymbol{G}_{4}^{\star}\right) \mathbf{1}, & \ddot{L}_{\varsigma \varsigma}=\mathbf{1}^{\top}\left(\boldsymbol{G}_{1} \boldsymbol{G}_{10}^{\star}-\left(\boldsymbol{G}_{1}^{\star}\right)^{2}\left(\boldsymbol{G}_{4}^{\star}\right)^{2}\right) \mathbf{1},
\end{aligned}
$$


As matrizes envolvidas são definidas a seguir

$$
\begin{aligned}
& \boldsymbol{G}_{5}^{\star}=\operatorname{diag}\left\{\frac{\partial^{2} D_{1}^{\star}(\boldsymbol{\theta})}{\partial^{2} \alpha}, \ldots, \frac{\partial^{2} D_{n}^{\star}(\boldsymbol{\theta})}{\partial^{2} \alpha}\right\}, \quad \boldsymbol{G}_{6 r}^{\star}=\operatorname{diag}\left\{\frac{\partial^{2} D_{1}^{\star}(\boldsymbol{\theta})}{\partial \alpha \partial \beta_{r}}, \ldots, \frac{\partial^{2} D_{n}^{\star}(\boldsymbol{\theta})}{\partial \alpha \partial \beta_{r}}\right\}, \\
& \boldsymbol{G}_{7}^{\star}=\operatorname{diag}\left\{\frac{\partial^{2} D_{1}^{\star}(\boldsymbol{\theta})}{\partial \alpha \partial \varsigma}, \ldots, \frac{\partial^{2} D_{n}^{\star}(\boldsymbol{\theta})}{\partial \alpha \partial \varsigma}\right\}, \quad \boldsymbol{G}_{8 r s}^{\star}=\operatorname{diag}\left\{\frac{\partial^{2} D_{1}^{\star}(\boldsymbol{\theta})}{\partial \beta_{r} \partial \beta_{s}}, \ldots, \frac{\partial^{2} D_{n}^{\star}(\boldsymbol{\theta})}{\partial \beta_{r} \partial \beta_{s}}\right\}, \\
& \boldsymbol{G}_{9 r}^{\star}=\operatorname{diag}\left\{\frac{\partial^{2} D_{1}^{\star}(\boldsymbol{\theta})}{\partial \beta_{r} \partial \varsigma}, \ldots, \frac{\partial^{2} D_{n}^{\star}(\boldsymbol{\theta})}{\partial \beta_{r} \partial \varsigma}\right\} \text { e } \boldsymbol{G}_{10}^{\star}=\operatorname{diag}\left\{\frac{\partial^{2} D_{1}^{\star}(\boldsymbol{\theta})}{\partial^{2} \varsigma}, \ldots, \frac{\partial^{2} D_{n}^{\star}(\boldsymbol{\theta})}{\partial^{2} \varsigma}\right\} .
\end{aligned}
$$

A estimação de parâmetros, predição do efeito aleatório e o teste do componente de variância do modelo 5.4 podem ser estendidas usando as Seções 4.3.5, 4.3.6 e 4.3.7, respectivamente.

\subsubsection{Influência local dos grupos}

Apresentamos a seguir de forma resumida os cálculos de influência local, isto é as matrices $\Delta$, sob os esquemas de perturbação de ponderação de casos, perturbação da resposta e perturbação de variáveis explicativas contínuas com base na Seção 4.3.8, em que $\gamma=\left[\alpha, \beta_{r}, \varsigma\right]^{\top}$, para $r=1, \ldots, p$.

\section{Ponderação de casos}

Matricialmente $\boldsymbol{\Delta}_{\gamma}=\left[\boldsymbol{\Delta}_{\alpha}^{\top}, \boldsymbol{\Delta}_{\beta_{r}}^{\top}, \boldsymbol{\Delta}_{\varsigma}^{\top}\right]^{\top}$ pode ser escrita da forma

$$
\boldsymbol{\Delta}_{\alpha}=\left(\boldsymbol{g}_{1}^{\star} \odot \boldsymbol{g}_{2}^{\star}\right)^{\top}, \quad \boldsymbol{\Delta}_{\beta_{r}}=\left(\boldsymbol{g}_{1}^{\star} \odot \boldsymbol{g}_{3 r}^{\star}\right)^{\top} \quad \text { e } \quad \boldsymbol{\Delta}_{\varsigma}=\left(\boldsymbol{g}_{1}^{\star} \odot \boldsymbol{g}_{4}^{\star}\right)^{\top},
$$

em que $\odot$ denota o produto Hadamard,

$$
\begin{aligned}
\boldsymbol{g}_{1}^{\star} & =\left[1 / D_{1}^{\star}(\boldsymbol{\theta}), \ldots, 1 / D_{n}^{\star}(\boldsymbol{\theta})\right]^{\top}, \\
\boldsymbol{g}_{2}^{\star} & =\left[\partial D_{1}^{\star}(\boldsymbol{\theta}) / \partial \alpha, \ldots, \partial D_{n}^{\star}(\boldsymbol{\theta}) / \partial \alpha\right]^{\top}, \\
\boldsymbol{g}_{3 r}^{\star} & =\left[\partial D_{1}^{\star}(\boldsymbol{\theta}) / \partial \beta_{r}, \ldots, \partial D_{n}^{\star}(\boldsymbol{\theta}) / \partial \beta_{r}\right]^{\top}, \quad \mathrm{e} \\
\boldsymbol{g}_{4}^{\star} & =\left[\partial D_{1}^{\star}(\boldsymbol{\theta}) / \partial \varsigma, \ldots, \partial D_{n}^{\star}(\boldsymbol{\theta}) / \partial \varsigma\right]^{\top} .
\end{aligned}
$$

\section{Perturbação aditiva da resposta}

Matricialmente $\boldsymbol{\Delta}_{i \gamma}=\left[\Delta_{i \alpha}, \Delta_{i \beta_{r}}, \Delta_{i \varsigma}\right]^{\top}$ pode ser escrita como

$$
\begin{aligned}
\Delta_{i \alpha} & =b_{i 1}^{\star}\left(\frac{\partial d_{i 1 \alpha}^{\star}}{\partial \omega_{i}}-b_{i 1}^{\star} c_{i 1}^{\star} d_{i 1 \alpha}^{\star}\right), \\
\Delta_{i \beta_{r}} & =b_{i 1}^{\star}\left(\frac{\partial d_{i 1 \beta_{r}}^{\star}}{\partial \omega_{i}}-b_{i 1}^{\star} c_{i 1}^{\star} d_{i 1 \beta_{r}}^{\star}\right), \quad \mathrm{e} \\
\Delta_{i \varsigma} & =b_{i 1}^{\star}\left(\frac{\partial d_{i 1 \varsigma}^{\star}}{\partial \omega_{i}}-b_{i 1}^{\star} c_{i 1}^{\star} d_{i 1 \varsigma}^{\star}\right),
\end{aligned}
$$

em que $b_{i 1}^{\star}=\frac{1}{D_{\omega_{i 1}}^{\star}(\boldsymbol{\theta})}, c_{i 1}^{\star}=\frac{\partial D_{\omega_{i 1}}^{\star}(\boldsymbol{\theta})}{\partial \omega_{i}}$ e $\boldsymbol{d}_{i 1 \gamma}^{\star}=\frac{\partial D_{\omega_{i 1}}^{\star}(\boldsymbol{\theta})}{\partial \boldsymbol{\gamma}}$. Detalhes podem ser consultados no Apêndice D. 


\section{Perturbação aditiva de uma variável explicativa}

Matricialmente $\boldsymbol{\Delta}_{i \gamma}=\left[\boldsymbol{\Delta}_{i \alpha}, \boldsymbol{\Delta}_{i \beta_{r}}, \boldsymbol{\Delta}_{i \varsigma}\right]^{\top}$ pode ser escrita como

$$
\begin{aligned}
\Delta_{i \alpha} & =b_{i 2}^{\star}\left(\frac{\partial d_{i 2 \alpha}^{\star}}{\partial \omega_{i}}-b_{i 2}^{\star} c_{i 2}^{\star} d_{i 2 \alpha}^{\star}\right), \\
\Delta_{i \beta_{r}} & =b_{i 2}^{\star}\left(\frac{\partial d_{i 2 \beta_{r}}^{\star}}{\partial \omega_{i}}-b_{i 2}^{\star} c_{i 2}^{\star} d_{i 2 \beta_{r}}^{\star}\right) \mathrm{e} \\
\Delta_{i \varsigma} & =b_{i 2}^{\star}\left(\frac{\partial d_{i 2 \varsigma}^{\star}}{\partial \omega_{i}}-b_{i 2}^{\star} c_{i 2}^{\star} d_{i 2 \varsigma}^{\star}\right),
\end{aligned}
$$

em que $b_{i 2}^{\star}=\frac{1}{D_{\omega_{i 2}^{\star}}^{\star}(\boldsymbol{\theta})}, c_{i 2}^{\star}=\frac{\partial D_{\omega_{i 2}}^{\star}(\boldsymbol{\theta})}{\partial \omega_{i}}$ e $\boldsymbol{d}_{i 2 \gamma}^{\star}=\frac{\partial D_{\omega_{i 2}}^{\star}(\boldsymbol{\theta})}{\partial \gamma}$. Detalhes podem ser consultados no Apêndice D.

\subsubsection{Resíduos}

Uma extensão natural do resíduo martingale definido na Seção 3.3.4 para os modelos log-BS-t mistos é a seguinte:

$$
r_{\mathrm{MT}_{i j}}=\operatorname{sign}\left(r_{\mathrm{M}_{i j}}\right) \sqrt{-2\left[r_{\mathrm{M}_{i j}}+\delta_{i j} \log \left(\delta_{i j}-r_{\mathrm{M}_{i j}}\right)\right]},
$$

em que $\delta_{i j}=0,1$ indica se a $j$-ésima observação do $i$-ésimo grupo é censurada ou não, respectivamente, enquanto

$$
r_{\mathrm{M}_{i j}}=\delta_{i j}+\log \left(\Phi_{t}\left(-\frac{2}{\widehat{\alpha}} \operatorname{senh}\left(\frac{y_{i j}-\widehat{\mu}_{i j}}{2}\right)\right)\right)
$$

é o resíduo martingale e $\widehat{\mu}_{i j}=\boldsymbol{x}_{i j}^{\top} \widehat{\boldsymbol{\beta}}+\tilde{b}_{i}$ com $\tilde{b}_{i}$ sendo a predição de $b_{i}$. Como $\widehat{\mu}_{i j}$ envolve também $\tilde{b}_{i}$ denominamos $r_{\mathrm{MT}_{i j}}$ como resíduo martingale condicional. O resíduo martingale marginal é definido da mesma forma, contudo $\widehat{\mu}_{i j}=\boldsymbol{x}_{i j}^{\top} \widehat{\boldsymbol{\beta}}$. Como foi observado na Seção 5.3.2 há indícios de robustez das estimativas de máxima verossimilhança $\widehat{\alpha}, \widehat{\beta}$ e $\widehat{\varsigma}$ contra observações aberrantes no sentido do resíduo $\widehat{\xi}_{i j k 2}$. Apresentamos a seguir um estudo entre o resíduo tipo martingale $r_{\mathrm{MT}_{i j}} \mathrm{e}$ a quantidade $\widehat{\xi}_{i j k 2}$ para $k$ fixado.

A Figura 5.1 descreve o comportamento de $r_{\mathrm{MT}_{i j}}$ contra o resíduo $\xi_{i j k 2}$ para observações não censuradas, para alguns valores de $\nu, \alpha=1$ e $\varsigma=1$. Podemos observar pela Figura 5.1 uma boa concordância entre os resíduos $r_{\mathrm{MT}_{i j}}$ e $\widehat{\xi}_{i j k 2}$, ou seja, valores altos para $\left|r_{\mathrm{MT}_{i j}}\right|$ implicam em valores altos para $\left|\widehat{\xi}_{i j k 2}\right|$. Como as estimativas de máxima verossimilhança $\widehat{\alpha}, \widehat{\beta}$ e $\widehat{\varsigma}$ são robustas contra valores altos de $\left|\widehat{\xi}_{i j k 2}\right|$ podemos dizer que essas estimativas são também robustas contra valores altos do resíduo tipo martingale $r_{\mathrm{MT}_{i j}}$. 


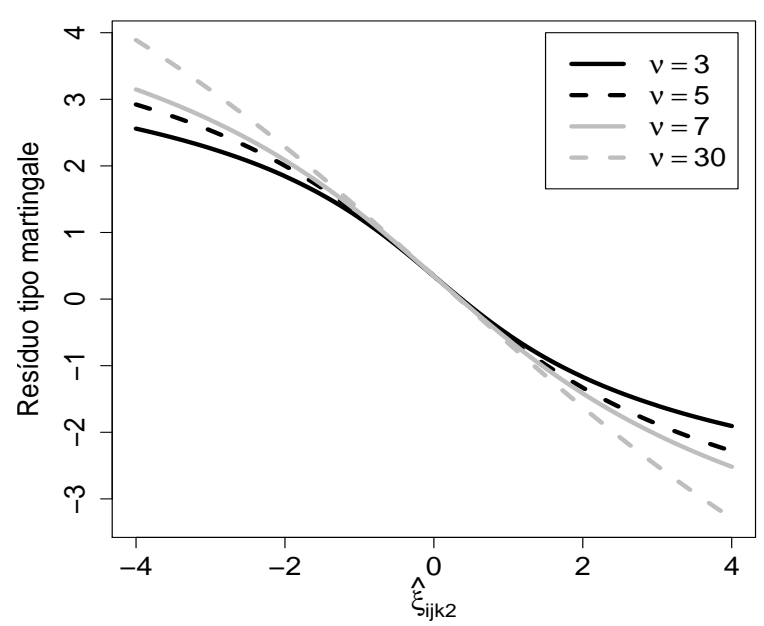

Figura 5.1: Comportamento do resíduo $r_{\mathrm{MT}_{i j}}$ contra o resíduo senh para observações não censuradas.

\subsection{Conclusões do capítulo}

Neste capítulo foram apresentados os modelos log-BS-t mistos para dados com censura, motivados pelo trabalho de Barros et al. (2008) em relação aos modelos log-BS-t de efeitos fixos já que as estimativas de máxima verossimilhança apresentam uma robustez em relação ao modelo log-BS de efeitos fixos. Em particular, estudamos os modelos log-BS-t de intercepto aleatório que serão usados no Capítulo 6. Usando a formulação marginal, aproximamos o logaritmo da função de verossimilhança baseado na quadratura de Gauss-Hermite não adaptativa e com isso foram calculadas a função escore e matriz hessiana. Alguns aspectos de robustez das estimativas de máxima verossimilhança, nas quais foram uma das principais contribuições deste trabalho, tem sido apresentadas. Além disso, análises de influência local (veja, por exemplo, Cook, 1986) e análises de resíduos tipo martingale condicional (veja, por exemplo, Barros et al., 2008) foram desenvolvidas. Seguindo os trabalhos de Lange et al. (1989), Fernandez e Steel (1999), Taylor e Verbyla (2004) e Leiva et al. (2008b) os graus de liberdade foram escolhidos com base no critério de informação de Akaike (AIC).

Analogamente ao Capítulo 2, os modelos log-BS-t mistos podem ser comparados aos modelos elípticos com efeitos mistos (veja, por exemplo, Osorio, 2006) sob o enfoque marginal no caso em que não se tem dados que apresentem algum tipo de censura. Isto acontece já que a distribuição log-BS-t apresenta em geral um comportamento simétrico, fato compartilhado pelos modelos simétricos univariados. 



\section{Capítulo 6}

\section{Aplicação}

Neste capítulo ajustamos os modelos log-Birnbaum-Saunders (log-BS) e log-Birnbaum-Saunders-t (log-BS-t) de efeitos fixos, como também os modelos log-BS e log-BS-t de intercepto aleatório a um conjunto de dados reais. Os quatro ajustes são comparados através das metodologias desenvolvidas neste trabalho.

\subsection{Descrição do problema}

Consideremos o conjunto da dados com censura tipo I estudado por Smith (1991) supondo um modelo de regressão Weibull. Esses dados correspondem aos tempos de falha de um tipo particular de fibra (Kevlar 49) submetido a quatro diferentes níveis de estresse medidos em MPa (Mega Pascal). Uma amostra de oito carretéis foi estudada, em que cada carretel foi submetido a diferentes níveis de estresse considerando réplicas. Os dados são apresentados na Tabela 6.5 no final deste capítulo.

Um gráfico de dispersão e boxplot dos tempos de falha para cada carretel são apresentados nas Figuras 6.1 e 6.2. Pode ser osbervar, com base nas figuras mencionadas anteriormente, que os tempos de falha apresentam uma assimetria positiva e que os maoires tempos são dos carretéis 1 e 5. Além disso, a Figura 6.1 mostra que estamos estudando dados com medidas repetidas que apresentam réplicas e que os carretéis 1, 4 e 8 possuim censura. Os dados de Smith (1991) apresentam $11 \%$ de censura. Como era de esperar (veja Figura 6.1), os tempos de falha decrescem quando os níveis de estresse aumentam.

Como foi mencionado no Capítulo 2, a distribuição Birnbaum-Saunders tem sido aplicada em estudos para relacionar o tempo de falha que apresenta algum tipo de dano cumulativo. Portanto usaremos a distribuição Birnbaum-Saunders para ajustar os dados de fibras (Kevlar 49). As análises são apresentadas a seguir.

\subsection{Modelo log-Birnbaum-Saunders de efeitos fixos}

Assumimos, inicialmente, que o tempo de falha possui uma distribuição Birnbaum-Saunders generalizada gerada por uma distribuição normal, ou seja, uma distribuição Birnbaum-Saunders clássica (Birnbaum e Saunders, 1969a). 


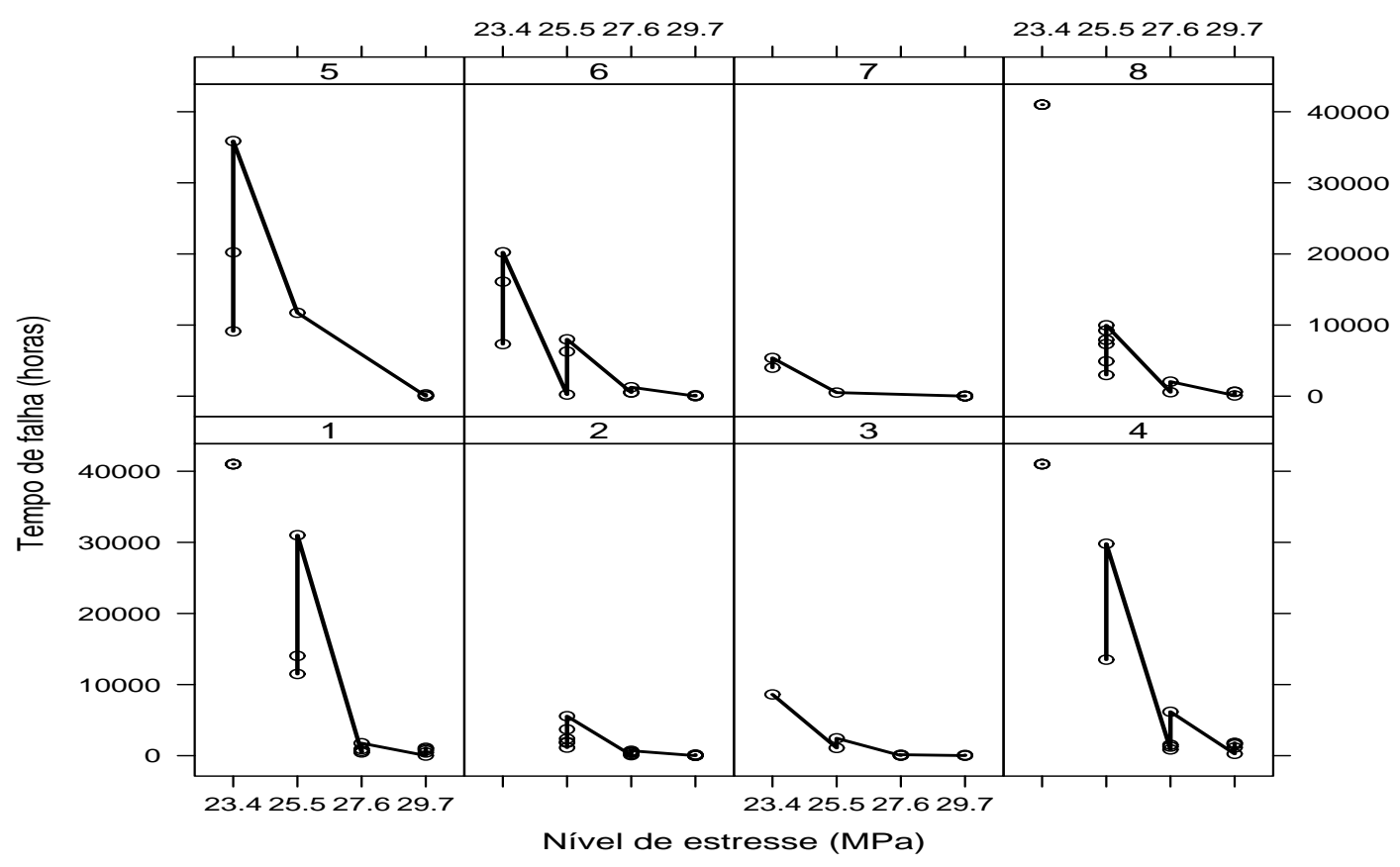

Figura 6.1: Gráfico de dispersão dos tempos de falha, para os dados de fibras.

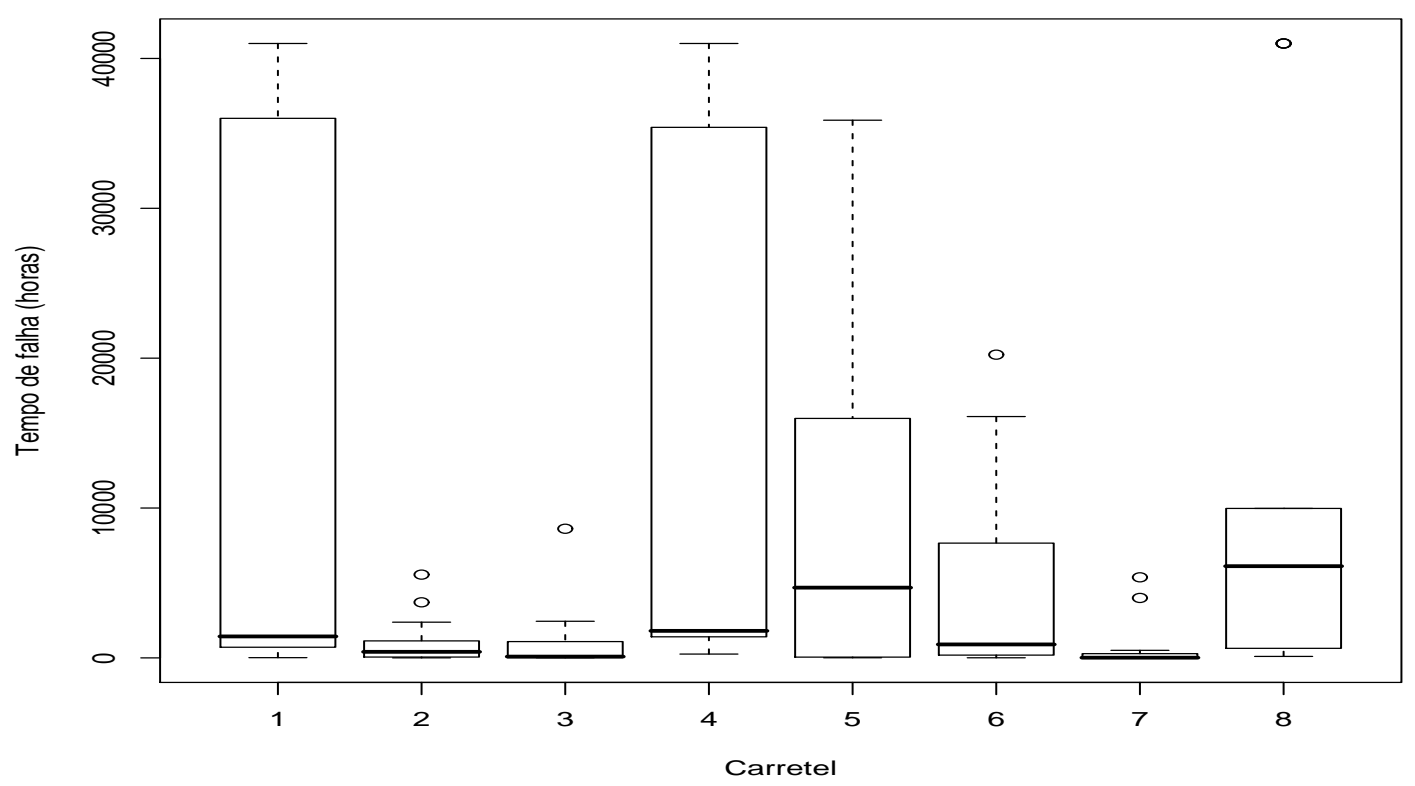

Figura 6.2: Boxplots dos tempos de falha para os dados de fibras (Kevlar 49). 


\subsubsection{Modelo log-BS}

A primeira abordagem para os dados de Smith (1991) é aplicar o modelo log-Birnbaum-Saunders de efeitos fixos com censura estudado por Leiva et al. (2007) dado por

$$
Y_{i j k}=\mu+\beta_{j}+\varepsilon_{i j k}, \quad i=1, \ldots, 8 ; \quad j=1, \ldots, 4 ; k=1, \ldots, m_{i j} \text { e } \quad \varepsilon_{i j} \sim \log -\mathrm{BS}(\alpha, 0),
$$

em que $Y_{i j k}$ denota o logaritmo do tempo de falha ou censura da $k$-ésima fibra submetida ao $j$-ésimo nível de estresse proveniente do $i$-ésimo carretel.

No modelo 6.1, $\mu$ representa a média geral de $Y_{i j k}, \beta_{1}=0$. Assim tem-se um modelo casela de referência em que $\beta_{2}$, $\beta_{3}$ e $\beta_{4}$ denotam os aumentos (ou diminuições) dos níveis de estresse 25,5, 27,6 e 29,7, respectivamente, em relação ao nível de estresse 23,4.

Para estimar os parâmetros do modelo usamos Proc NLMIXED do SAS. As saídas computacionais são apresentadas na Tabela 6.1.

Tabela 6.1: Estimativas dos parâmetros do modelo log-Birnbaum-Saunders de efeitos fixos ajustado aos dados de fibras e seus correspondentes níveis de significância.

\begin{tabular}{ccccc}
\hline Efeito & Estimativa & E. Padrão & $\mathbf{Z}$ & Valor $\mathbf{p}$ \\
\hline Intercepto & 11,0462 & 0,3985 & 27,7186 & $<0,0001$ \\
estresse $_{25,5}$ & $-2,9271$ & 0,5798 & $-5,0484$ & $<0,0001$ \\
estresse $_{27,6}$ & $-5,2909$ & 0,5336 & $-9,9159$ & $<0,0001$ \\
estresse $_{29,7}$ & $-6,7854$ & 0,4320 & $-15,7058$ & $<0,0001$ \\
\hline$\alpha$ & 2,1477 & 0,1623 & 13,2293 & $<0,0001$ \\
\hline
\end{tabular}

$\ell(\hat{\boldsymbol{\theta}})=-187,7757$.

Podemos observar da Tabela 6.1 que por exemplo, $\hat{\beta}_{2}=-2,9271$, isto é, o logaritmo do tempo de falha teve uma diminuição do nível de estresse 23,4 para o nível 25,5 . Isso ocorre também do nível 23,4 para o nível 27,6 e do nível 23,4 para o nível 29,7. O valor de $\hat{\alpha}=2,1477$ significa que a distribuição condicional do tempo de falha (dado o nível de estresse) é fortemente assimétrica à direita. Todos os parâmetros são significativos.

Para avaliar afastamentos das suposições do modelo (6.1), realizamos uma análise de resíduos e de influência local (ponderação de casos), veja as Figuras 6.3 e 6.4, respectivamente para mais detalhes. Pode-se observar na Figura 6.4 que as observações \#16, \#30 e \#62 apresentam um comportamento afastado da maioria das observações. Em particular os dados mencionados anteriormente correspondem aos logaritmos dos tempos de falha submetidos ao nível de estresse 29,7 (MPa) em que a variável de interesse apresentou um valor alto, baixo e alto, respectivamente. Já que observações atípicas foram observadas quando o modelo (6.1) foi ajustado, vamos reanalisar os dados considerando uma distribuição Birnbaum-Saunders generalizada gerada por uma distribuição t-Student, ou simplesmente Birnbaum-Saunders t. Os graus de liberdade foram escolhidos baseando-se no critério de informação de Akaike (AIC), e o valor obtido para $\nu$ foi $\nu=10$. 

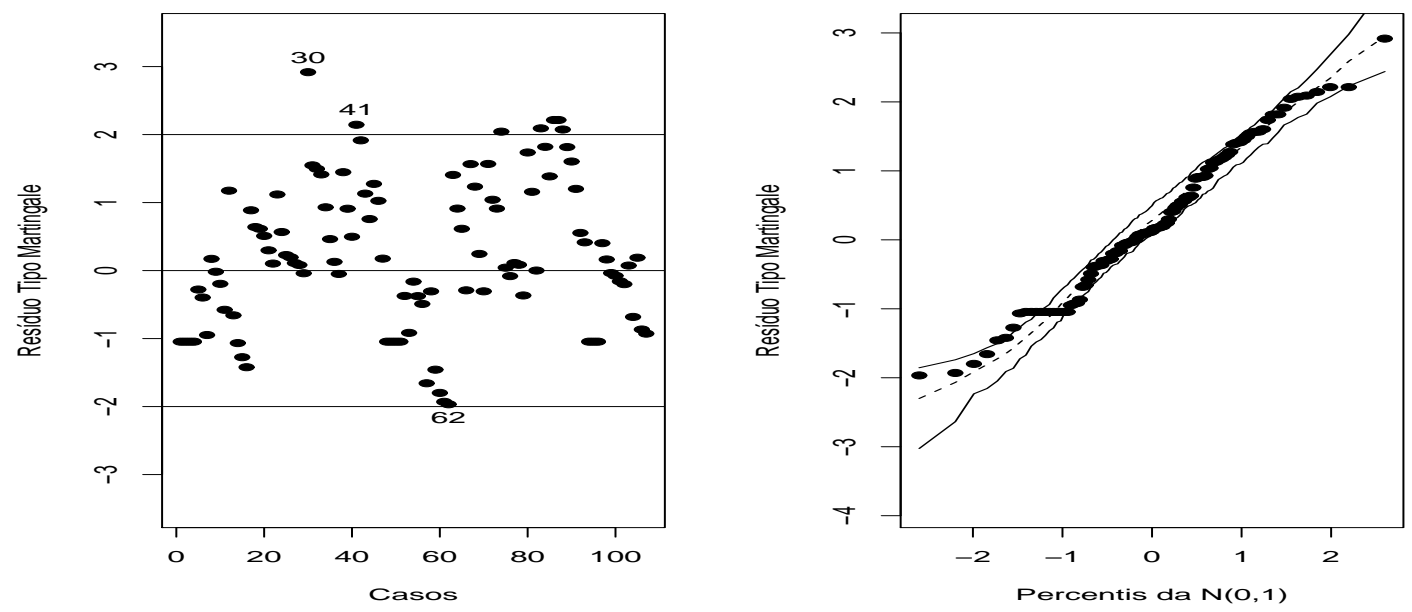

Figura 6.3: Gráfico de índices (esquerda) e gráfico normal de probabilidades do resíduo tipo Martingale, modelo log-Birnbaum-Saunders.

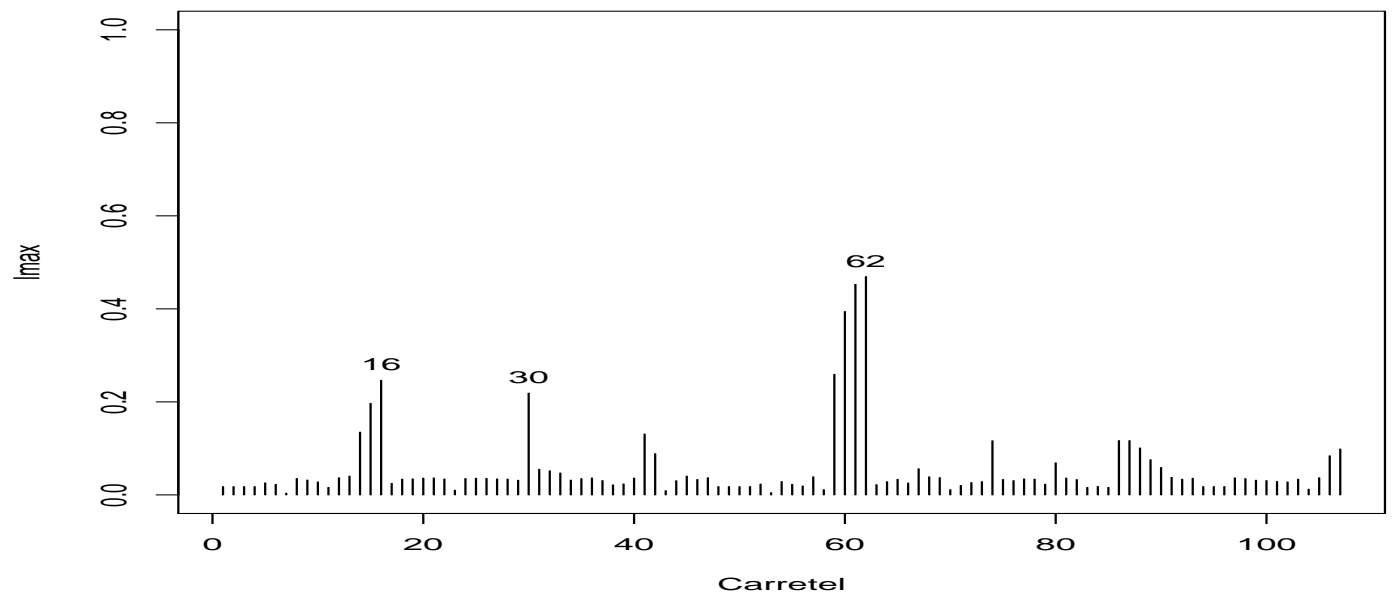

Figura 6.4: Gráfico de índices de $l_{\max }$ para $\boldsymbol{\theta}$ considerando o modelo log-Birnbaum-Saunders.

\subsubsection{Modelo log-BS-t}

Consideremos o seguinte modelo log-Birnbaum-Saunders-t de efeitos fixos com censura aleatória, proposto por Barros et al. (2008):

$$
Y_{i j k}=\mu+\beta_{j}+\varepsilon_{i j}, \quad i=1, \ldots, 8 ; j=1, \ldots, 4 ; k=1, \ldots, m_{i j} \text { e } \quad \varepsilon_{i j} \sim \log \text {-BS-t }(\alpha, 0 ; 10),(6.2)
$$

em que $Y_{i j k}$ denota o logaritmo do tempo de falha ou censura da $k$-ésima fibra submetida ao $j$ ésimo nível de estresse proveniente do $i$-ésimo carretel, como foi dito anteriormente. A estimação dos parâmetros do modelo (6.2) foi feita usando o Proc NLNIXED do SAS. Os resultados são 
apresentadas na Tabela 6.2. Nota-se pela Tabela 6.2 uma similaridade entre as estimativas dos modelos log-BS e log-BS-t para os efeitos dos níveis de estresse, confirmando os resultados da análise descritiva, em que o logaritmo do tempo de falha tende a decrescer com o aumento do nível de estresse.

Tabela 6.2: Estimativas dos parâmetros do modelo log-Birnbaum-Saunders-t de efeitos fixos ajustado aos dados de fibras e seus correspondentes níveis de significância.

\begin{tabular}{ccccc}
\hline Efeito & Estimativa & E. Padrão & $\mathbf{Z}$ & Valor p \\
\hline Intercepto & 10,9697 & 0,4180 & 26,24 & $<0,0001$ \\
estresse $_{25,5}$ & $-2,7537$ & 0,5814 & $-4,74$ & $<0,0001$ \\
estresse $_{27,6}$ & $-5,1403$ & 0,5611 & $-9,16$ & $<0,0001$ \\
estresse $_{29,7}$ & $-6,7448$ & 0,4615 & $-14,61$ & $<0,0001$ \\
\hline$\alpha$ & 1,9631 & 0,1667 & 11,78 & $<0,0001$ \\
\hline
\end{tabular}

$\ell(\hat{\boldsymbol{\theta}})=-188,6310$.

A seguir apresentamos alguns resultados obtidos a partir das análises de diagnóstico, em particular análise de resíduos e influência local (ponderação de casos), para estudar se há observações atípicas e se as suposições feitas no modelo são adequadas (veja Figuras 6.5 e 6.6). Baseando-se na Figura 6.5 parece ser razoável a suposição de que os dados seguem uma distribuição BirnbaumSaunders e também podemos observar que as observações \#30, \#41 e \#74 possuim valores altos para o resíduo tipo martingale. Pode-se observar da Figura 6.6 que o modelo log-Birnbaum-Saunders-t diminui a influência da observação \#30, porém alguns pontos como \#16 e \#32 continuam a se destacar dos demais. Devido ao fato dos dados de Smith (1991) serem dados de medidas repetidas ajustaremos a seguir os modelos log-BS de intercepto aleatório em que estudaremos se os carreteis são da mesma população ou não.

\subsection{Modelo $\log$-BS de intercepto aleatório}

Nesta seção reanalisamos os dados de Smith (1991) sob modelos de intercepto aleatório.

\subsubsection{Modelo log-BS}

Consideremos o seguinte modelo log-Birnbaum-Saunders misto:

$$
\begin{aligned}
Y_{i j k} \mid b_{i} & \stackrel{\text { ind }}{\sim} \log -\mathrm{BS}\left(\alpha, \mu_{i j}\right), \\
b_{i} & \stackrel{\mathrm{iid}}{\sim} \mathcal{N}(0, \varsigma), \quad i=1, \ldots, 8 ; j=1, \ldots, 4 \text { e } k=1, \ldots, m_{i j},
\end{aligned}
$$

em que $Y_{i j k}$ denota o logaritmo do tempo de falha ou censura da $k$-ésima fibra submetida ao $j$-ésimo nível de estresse proveniente do $i$-ésimo carretel, $\mu_{i j}=\mu+b_{i}+\beta_{j}$ e $b_{i}$ representa o efeito aleatório associado ao $i$-ésimo carretel. O número de réplicas para cada carretel foi 16, 21, 10, 15 , 8, 12, 11 e 14, respectivamente. Portanto, temos um modelo de medidas repetidas desbalanceado. As estimativas de máxima verossimilhança dos parâmetros do modelo (6.3) foram calculados baseadas no logaritmo da função de verossimilhança, a qual foi aproximada pela quadratura 

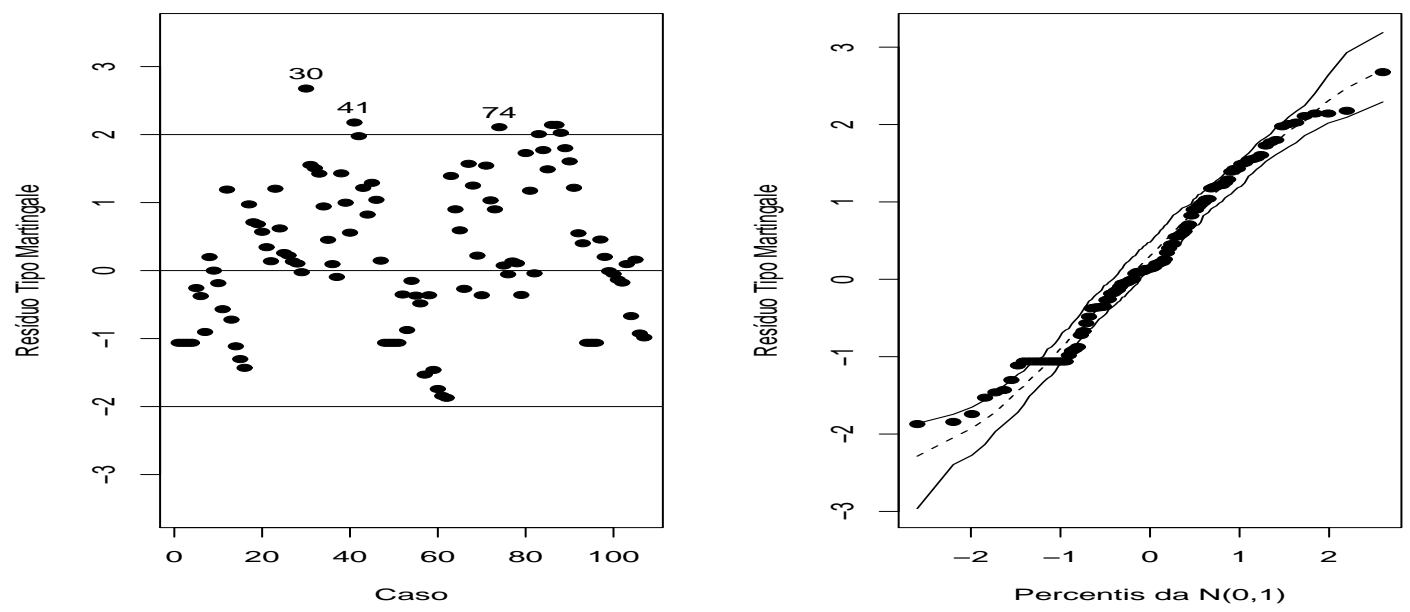

Figura 6.5: Gráfico de índices (esquerda) e gráfico normal de probabilidades do resíduo tipo Martingale, modelo log-Birnbaum-Saunders-t.

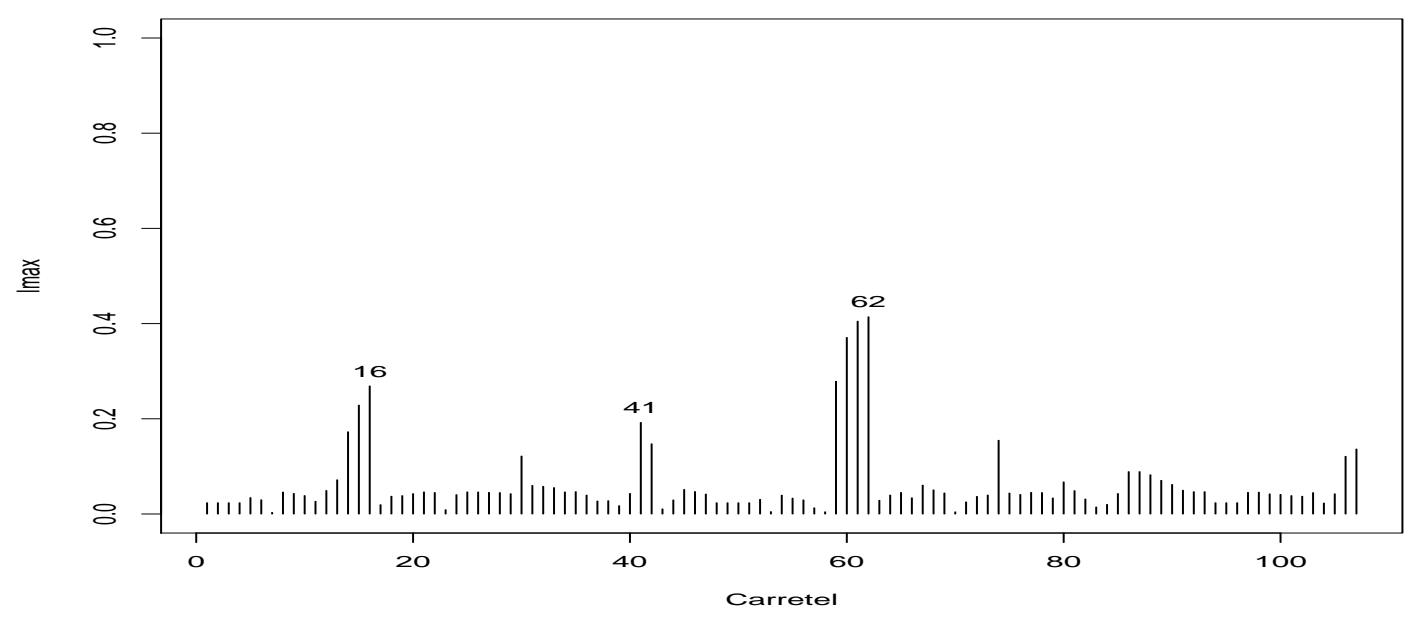

Figura 6.6: Gráfico de índices de $l_{\max }$ para $\boldsymbol{\theta}$ considerando o modelo log-Birnbaum-Saunders-t.

de Gauss Hermite com 50 pontos de quadratura. O algoritmo para estimar os parâmetros foi o algoritmo de quase-Newton. O programa para estimar os parâmetros do modelo foi o SAS, usando o Proc NLMIXED cujo código pode ser consultado no Apêndice E. A saída computacional do SAS é apresentada na Tabela 6.3.

Podemos observar pela Tabela 6.3 que todas as estimativas são significativas. Baseado no teste da componente de variância ( $\mathrm{p}=0,038$ ), rejeitamos a hipótese nula, isto é, o efeito aleatório deve ser incluído no modelo, o que indica que os oito carretéis parecem vir de diferentes populações. A seguir estudamos os resíduos do modelo (6.3); ver Figura 6.7. Analogamente à Seção 6.2, reanalisamos o modelo log-BS de intercepto aleatório considerando uma distribuição log-BS-t $\left(\alpha, \mu_{i j} ; 10\right)$. 
Tabela 6.3: Estimativas dos parâmetros do modelo log-Birnbaum-Saunders de intercepto aleatório ajustado para os dados de fibras e seus correspondentes níveis de significância.

\begin{tabular}{ccccc}
\hline Efeito & Estimativa & E. Padrão & $\mathbf{Z}$ & Valor p \\
\hline Intercepto & 10,7707 & 0,4860 & 22,1619 & $<0,0001$ \\
estresse $_{25,5}$ & $-2,3807$ & 0,4329 & $-5,4994$ & $<0,0001$ \\
estresse $_{27,6}$ & $-4,6366$ & 0,4391 & $-10,5593$ & $<0,0001$ \\
estresse $_{29,7}$ & $-6,4615$ & 0,3921 & $-16,4834$ & $<0,0001$ \\
\hline$\alpha$ & 1,2154 & 0,0951 & 12,7802 & $<0,0001$ \\
\hline
\end{tabular}

$\ell(\hat{\boldsymbol{\theta}})=-157,00$ e $\hat{\varsigma}=1,8644(1,0529)$.
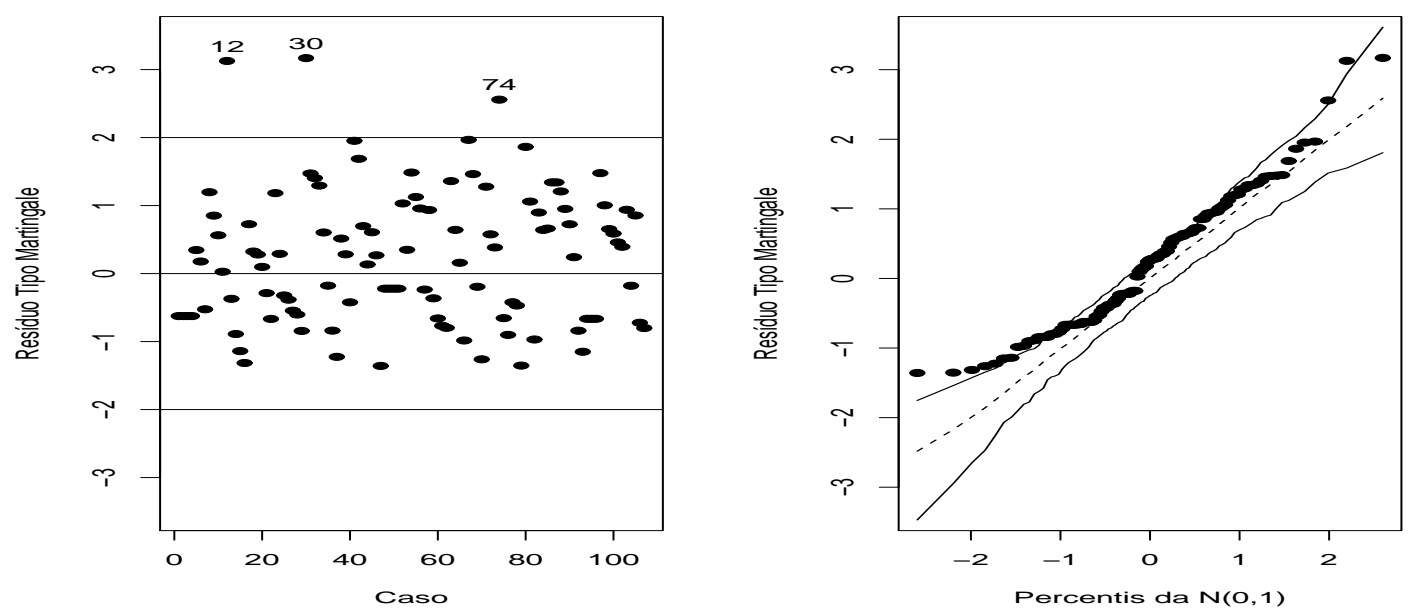

Figura 6.7: Gráfico de índices do resíduo tipo Martingale (esquerda) e gráfico normal de probabilidades (direita) para o modelo log-Birnbaum-Saunders de intercepto aleatório.

\subsubsection{Modelo log-BS-t}

Consideremos o seguinte modelo, log-Birnbaum-Saunders-t misto:

$$
\begin{aligned}
Y_{i j k} \mid b_{i} & \stackrel{\text { ind }}{\sim} \log \text {-BS-t }\left(\alpha, \mu_{i j} ; 10\right), \\
b_{i} & \stackrel{\text { iid }}{\sim} \mathcal{N}(0, \varsigma), \quad i=1, \ldots, 8 ; j=1, \ldots, 4 \text { e } k=1, \ldots, m_{i j},
\end{aligned}
$$

em que $Y_{i j k}, \mu_{i j}$ e $b_{i}$ foram definidas na equação (6.3). O ajuste do modelo foi feito usando o Proc NLMIXED do SAS, cujo código pode ser consultado no Apêndice E. Os resultados do ajuste se encontram na Tabela 6.4. Pode-se observar da Tabela 6.4 que a estimativa de $\alpha$ sob o modelo $\log$-BS-t de intercepto aleatório é aproximadamente $10 \%$ menor do que sob o modelo log-BS de intercepto aleatório. Como essa estimativa está relacionada com a assimetria da distribuição condicional do tempo de falha, podemos dizer que o uso de um modelo de caudas mais pesadas pode ter reduzido a influência das observações mais aberrantes na estimativa de $\alpha$.

A Figura 6.8 mostra que o modelo log-Birnbaum-Saunders-t de intercepto aleatório não diminui a influência das observações \#12, \#30, \#47 e \#74. 
Tabela 6.4: Estimativas dos parâmetros do modelo log-Birnbaum-Saunders-t de intercepto aleatório ajustado para os dados de fibras e seus correspondentes níveis de significância.

\begin{tabular}{ccccc}
\hline Efeito & Estimativa & E. Padrão & $\mathbf{Z}$ & Valor p \\
\hline Intercepto & 10,6004 & 0,5261 & 20,1490 & $<0,0001$ \\
estresse $_{25,5}$ & $-2,2538$ & 0,4111 & $-5,4824$ & $<0,0001$ \\
estresse $_{27,6}$ & $-4,5650$ & 0,4158 & $-10,9788$ & $<0,0001$ \\
estresse $_{29,7}$ & $-6,2892$ & 0,3782 & $-16,6293$ & $<0,0001$ \\
\hline$\alpha$ & 1,0985 & 0,0954 & 11,5135 & $<0,0001$ \\
\hline
\end{tabular}

$\ell(\hat{\boldsymbol{\theta}})=-156,90, \hat{\varsigma}=1,5673(0,7291)$.
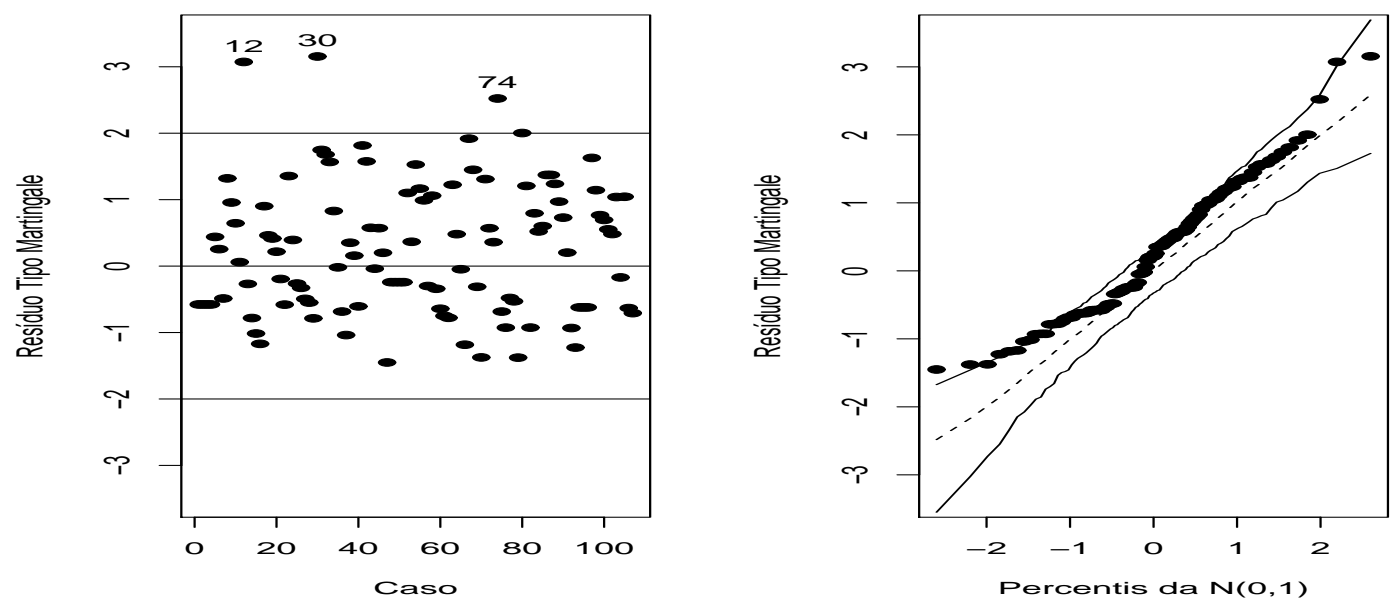

Figura 6.8: Gráfico de índice do Resíduo tipo Martingale (esquerda) e gráfico normal de probabilidades (direita) para o modelo log-Birnbaum-Saunders-t de intercepto aleatório.

\subsection{Conclusões da aplicação}

O teste da componente de variância, para decidir se o efeito aleatório deve ser considerado no modelo, foi estudado para o modelo (6.3) o qual teve um valor $\mathrm{p}=0,038$. Logo podemos concluir que os carretéis vêm de diferentes populações. Analogamente, para o modelo (6.4) o teste da componente de variância rejeitou a hipótese de que os carretéis vêm da mesma população ( valor $\mathrm{p}=0,016$ ). Como os resultados sob o modelo $\log$-BS-t de intercepto aleatório não foram muito diferentes dos resultados sob o modelo log-BS de intercepto aleatório, escolhemos o modelo mais simples, isto é, o modelo log-BS de intercepto aleatório. Concluímos a nossa análise apresentando o gráfico dos perfis estimados (veja Figura 6.9) para os dados de fibras (Kevlar 49, Smith (1991)) para os tempos e o logaritmos dos tempos sob o modelo log-BS de intercepto aleatório. 

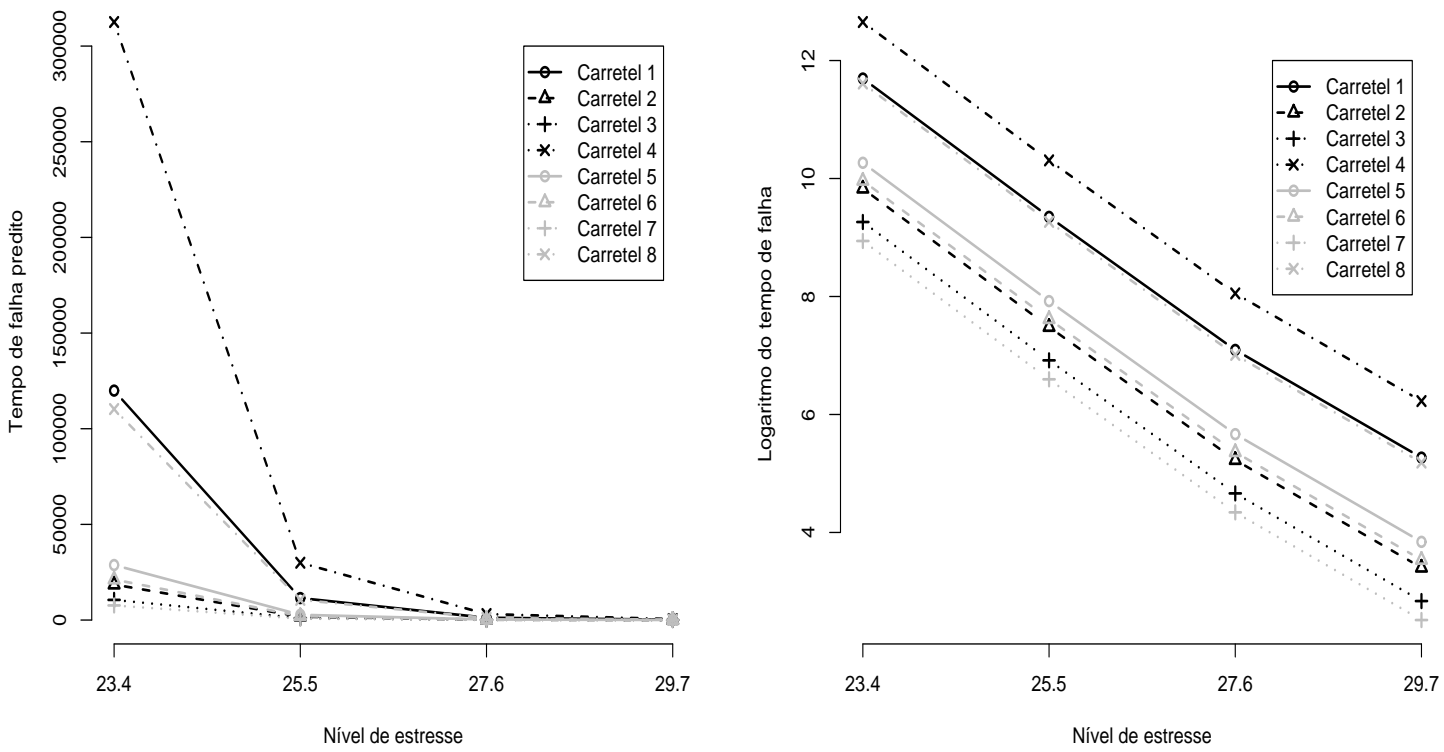

Figura 6.9: Gráficos de perfis ajustado para o tempo de falha (esquerda) e para o logaritmo do tempo de falha (direita) sob o modelo log-BS de intercepto aleatório. 
Tabela 6.5: Logaritmos dos tempos de falha de um tipo particular de fibra (Kevlar 49) segundo o nível de estresse e ocorrência ou não de censura.

\begin{tabular}{|c|c|c|c|c|c|c|c|c|c|c|c|c|c|c|c|}
\hline Grupo & $\mathrm{x}$ & $\delta$ & $\mathrm{y}$ & Grupo & $\mathbf{x}$ & $\delta$ & $\mathrm{y}$ & Grupo & $\mathrm{x}$ & $\delta$ & $\mathrm{y}$ & Grupo & $\mathrm{x}$ & $\delta$ & $\mathrm{y}$ \\
\hline 1 & 23,4 & 0 & 10,6213 & 2 & 27,6 & 1 & 6,3006 & 4 & 27,6 & 1 & 7,1512 & 6 & 29,7 & 1 & 4,9698 \\
\hline 1 & 23,4 & 0 & 10,6213 & 2 & 27,6 & 1 & 6,5426 & 4 & 27,6 & 1 & 7,3375 & 7 & 23,4 & 1 & 8,2940 \\
\hline 1 & 23,4 & 0 & 10,6213 & 2 & 29,7 & 1 & 0,7885 & 4 & 27,6 & 1 & 8,7287 & 7 & 23,4 & 1 & 8,5897 \\
\hline 1 & 23,4 & 0 & 10,6213 & 2 & 29,7 & 1 & 2,1401 & 4 & 29,7 & 1 & 5,5377 & 7 & 25,5 & 1 & 6,2218 \\
\hline 1 & 25,5 & 1 & 9,3490 & 2 & 29,7 & 1 & 2,2083 & 4 & 29,7 & 1 & 7,0462 & 7 & 29,7 & 1 & 1,3863 \\
\hline 1 & 25,5 & 1 & 9,5491 & 2 & 29,7 & 2 & 3,3224 & 4 & 29,7 & 1 & 7,3584 & 7 & 29,7 & 1 & 1,3863 \\
\hline 1 & 25,5 & 1 & 10,3420 & 2 & 29,7 & 1 & 3,0956 & 4 & 29,7 & 1 & 7,4677 & 7 & 29,7 & 1 & 1,5261 \\
\hline 1 & 27,6 & 1 & 6,1168 & 2 & 29,7 & 1 & 4,0146 & 4 & 29,7 & 1 & 7,4967 & 7 & 29,7 & 1 & 1,8083 \\
\hline 1 & 27,6 & 1 & 6,4990 & 2 & 29,7 & 1 & 4,7131 & 5 & 23,4 & 1 & 9,1182 & 7 & 29,7 & 1 & 2,0669 \\
\hline 1 & 27,6 & 1 & 6,8356 & 2 & 29,7 & 1 & 5,0670 & 5 & 23,4 & 1 & 9,9150 & 7 & 29,7 & 1 & 2,6391 \\
\hline 1 & 27,6 & 1 & 7,4705 & 3 & 23,4 & 1 & 9,0614 & 5 & 23,4 & 1 & 10,4879 & 7 & 29,7 & 1 & 3,8265 \\
\hline 1 & 29,7 & 1 & 2,6810 & 3 & 25,5 & 1 & 6,9918 & 5 & 25,5 & 1 & 9,3697 & 7 & 29,7 & 1 & 4,1141 \\
\hline 1 & 29,7 & 1 & 6,0960 & 3 & 25,5 & 1 & 7,8008 & 5 & 29,7 & 1 & 2,1163 & 8 & 23,4 & 0 & 10,6213 \\
\hline 1 & 29,7 & 1 & 6,6270 & 3 & 27,6 & 1 & 2,9497 & 5 & 29,7 & 1 & 2,5878 & 8 & 23,4 & 0 & 10,6213 \\
\hline 1 & 29,7 & 1 & 6,8588 & 3 & 27,6 & 1 & 3,1905 & 5 & 29,7 & 1 & 4,4716 & 8 & 23,4 & 0 & 10,6213 \\
\hline 1 & 29,7 & 1 & 7,0105 & 3 & 27,6 & 1 & 4,2456 & 5 & 29,7 & 1 & 5,5369 & 8 & 25,5 & 1 & 7,9979 \\
\hline 2 & 25,5 & 1 & 7,0338 & 3 & 27,6 & 1 & 4,9127 & 6 & 23,4 & 1 & 8,8984 & 8 & 25,5 & 1 & 8,4988 \\
\hline 2 & 25,5 & 1 & 7,5090 & 3 & 29,7 & 1 & 2,5257 & 6 & 23,4 & 1 & 9,6868 & 8 & 25,5 & 1 & 8,9000 \\
\hline 2 & 25,5 & 1 & 7,5601 & 3 & 29,7 & 1 & 2,9285 & 6 & 23,4 & 1 & 9,9151 & 8 & 25,5 & 1 & 8,9770 \\
\hline 2 & 25,5 & 1 & 7,7761 & 3 & 29,7 & 1 & 4,6151 & 6 & 25,5 & 1 & 5,4170 & 8 & 25,5 & 1 & 9,1313 \\
\hline 2 & 25,5 & 1 & 8,2185 & 4 & 23,4 & 0 & 10,6213 & 6 & 25,5 & 1 & 8,7437 & 8 & 25,5 & 1 & 9,2076 \\
\hline 2 & 25,5 & 1 & 8,6226 & 4 & 23,4 & 0 & 10,6213 & 6 & 25,5 & 1 & 8,9867 & 8 & 27,6 & 1 & 6,3175 \\
\hline 2 & 27,6 & 1 & 4,2655 & 4 & 23,4 & 0 & 10,6213 & 6 & 27,6 & 1 & 6,2426 & 8 & 27,6 & 1 & 7,6237 \\
\hline 2 & 27,6 & 1 & 5,2938 & 4 & 23,4 & 0 & 10,6213 & 6 & 27,6 & 1 & 6,2945 & 8 & 29,7 & 1 & 4,5870 \\
\hline 2 & 27,6 & 1 & 6,0007 & 4 & 25,5 & 1 & 9,5105 & 6 & 27,6 & 1 & 7,1348 & 8 & 29,7 & 1 & 6,3808 \\
\hline 2 & 27,6 & 1 & 6,0689 & 4 & 25,5 & 1 & 10,3025 & 6 & 29,7 & 1 & 1,9021 & 8 & 29,7 & 1 & 6,4580 \\
\hline 2 & 27,6 & 1 & 6,2424 & 4 & 27,6 & 1 & 6,7762 & 6 & 29,7 & 1 & 2,7081 & & & & \\
\hline
\end{tabular}




\section{Capítulo 7}

\section{Considerações Finais}

\subsection{Conclusões}

Neste trabalho estudamos os modelos log-Birnbaum-Saunders e log-Birnbaum-Saunders-t de efeitos aleatórios para dados que apresentam censura. Em particular, foram estendidos os trabalhos de Rieck e Nedelman (1991), Galea et al. (2004), Leiva et al. (2007) e Barros et al. (2008). Derivamos as matrizes associadas ao método de influência local proposto por Cook (1986) e analisamos os resíduos tipo martingale (Therneau et al., 1990; Ortega, 2001; Ortega et al., 2003; Leiva et al., 2007).

Aplicamos os modelos log-Birnbaum-Saunders e log-Birnbaum-Saunders-t de intercepto aleatório a um conjunto de dados reais (Smith, 1991), utilizando o Proc NLMIXED do SAS (Littell et al., 1996).

Utilizamos os métodos de integração numérica, em particular a quadratura de Gauss-Hermite, para aproximar o logaritmo da função de verossimilhança dos modelos com intercepto aleatório. Mostramos que existe uma relação entre a quadratura de Gauss-Hermite adaptativa (Pinheiro e Bates, 1995; Pinheiro e Chao, 2006) e a aproximação de Laplace (Tierney e Kadane, 1986; Leonard et al., 1989). Com base na quadratura de Gauss-Hermite calculamos as funções escore e as matrizes de informação observadas para os parâmetros dos modelos log-BS e log-BS-t intercepto aleatório.

\subsection{Perspectivas para trabalhos futuros}

Abaixo apresentamos uma lista de alguns problemas que podem ser de interesse para futuras pesquisas:

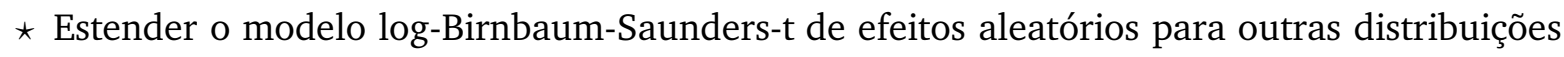
simétricas.

^ Estudar outros tipos de aproximação para o logaritmo da função de verossimilhança, tais como, importance sampling e Monte Carlo (Tanner, 1996) e compará-los com a quadratura de Gauss-Hermite (Pinheiro e Bates, 1995; Pinheiro e Chao, 2006). 
* Usar o algoritmo EM (Dempster et al., 1977) para estimar os parâmetros dos modelos logBirnbaum-Saunders de efeitos aleatórios.

* Utilizar a distância de Cook e alavanca generalizada como medidas de influência global (Cook e Weisberg, 1982; Laurent e Cook, 1992; Wei et al., 1998). 


\section{Apêndice A}

\section{Código $\mathrm{R}$ baseado no pacote bs}

\section{A.1 Aspectos computacionais}

Nesta seção apresentamos duas funções que dependem do pacote bs do R (Leiva et al., 2006), isto é graphdbs e grapfrbs, as quais foram utilizadas para gerar as Figuras 2.1 e 1.2, respectivamente. Modificando levemente as duas funções mencionadas anteriormente, temos que o código no R, fica expresso da seguinte forma:

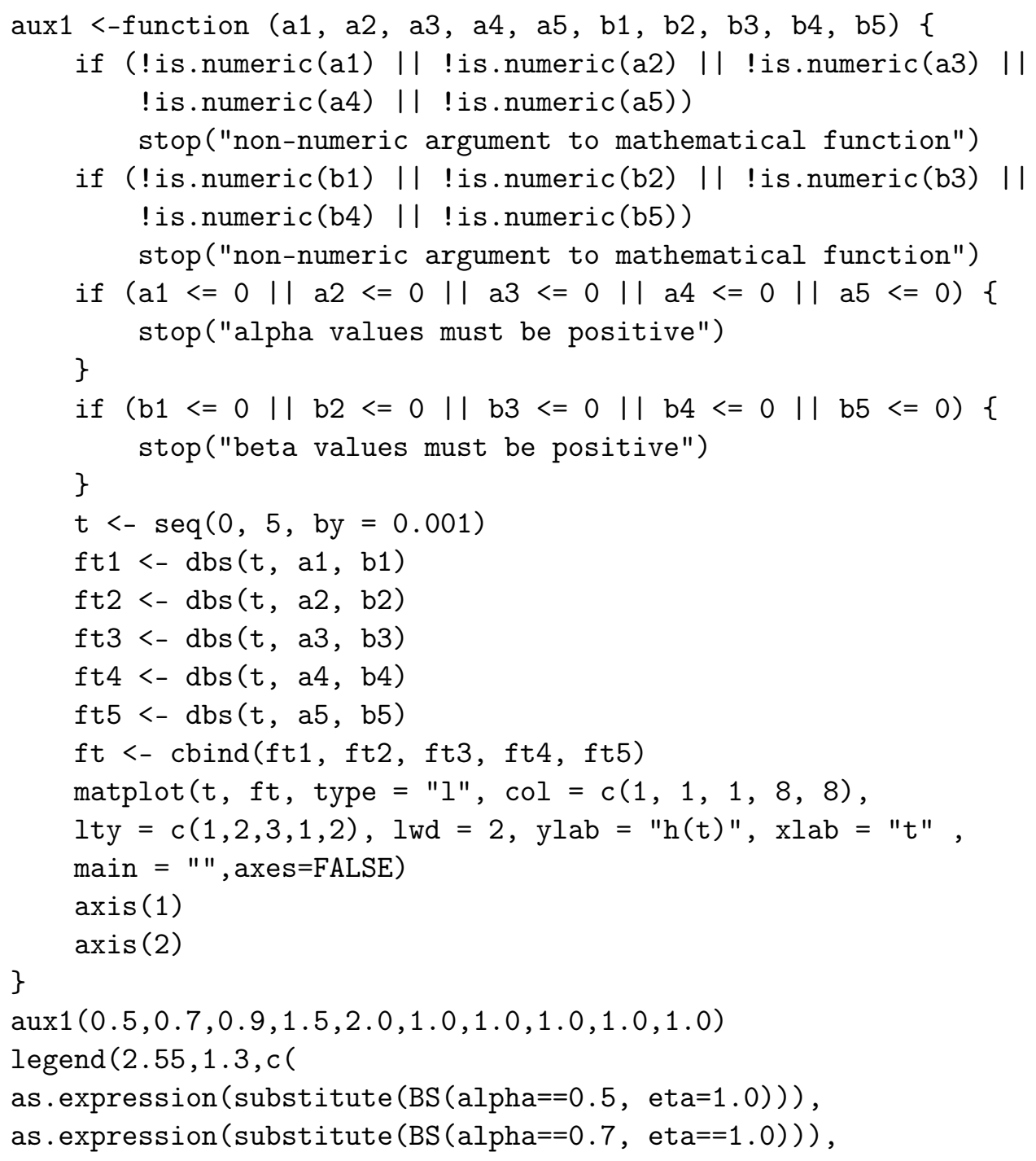




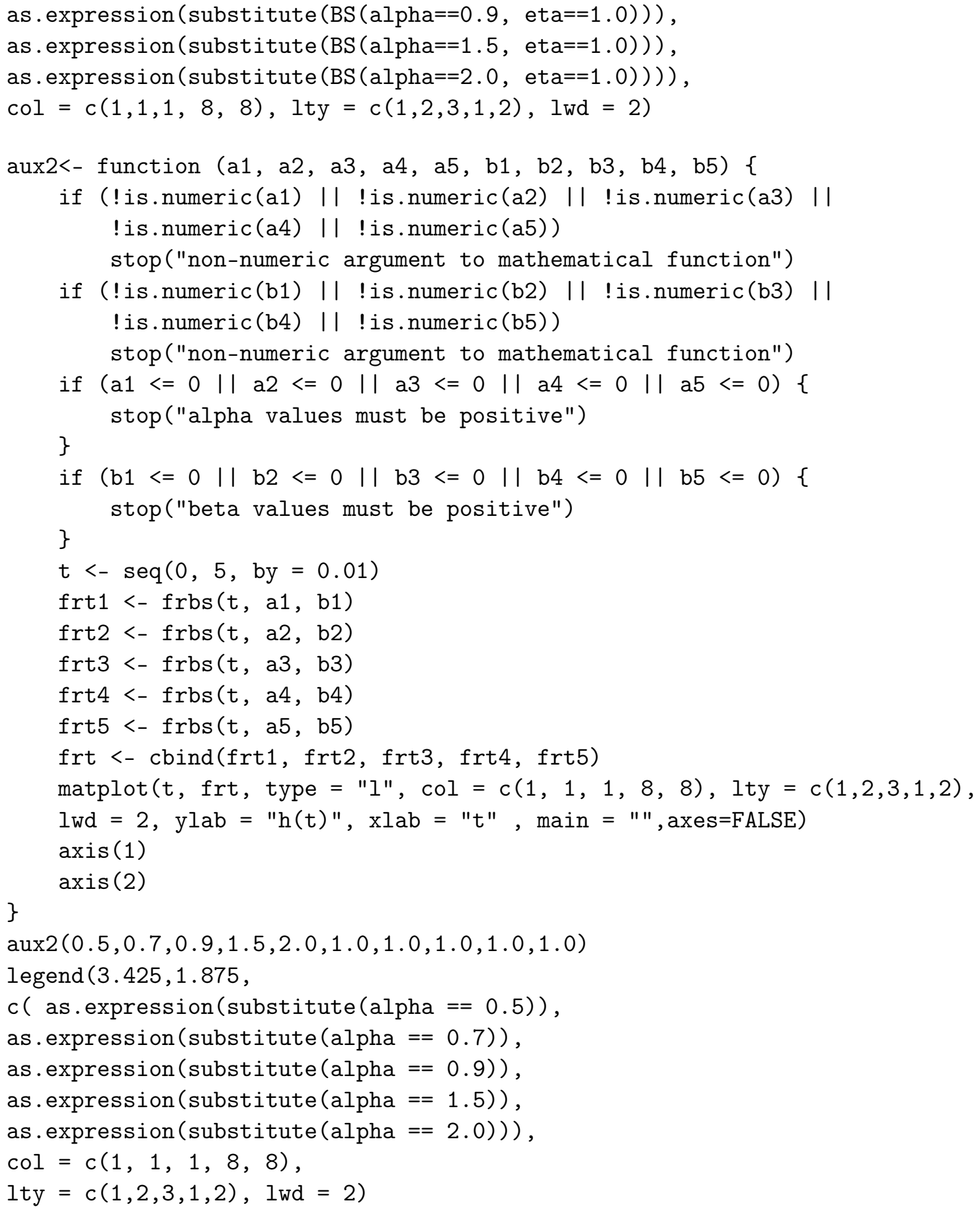




\section{Apêndice $B$}

\section{Derivadas usadas nos Capítulos 4 e 5}

Neste apêndice são apresentadas as derivadas do que foram usadas para calcular o vetor escore para os modelos log-Birnbaum-Saunders e log-Birnbaum-Saunders-t de intercepto aleatório.

\section{B.1 Cálculos}

Começamos definindo algumas expressões

$$
\begin{aligned}
& \xi_{i j k 1}=\frac{2}{\alpha} \cosh \left(\frac{y_{i j}-\boldsymbol{x}_{i j}^{\top} \boldsymbol{\beta}-\sqrt{2 \varsigma} s_{k}}{2}\right) \\
& \xi_{i j k 2}=\frac{2}{\alpha} \operatorname{senh}\left(\frac{y_{i j}-\boldsymbol{x}_{i j}^{\top} \boldsymbol{\beta}-\sqrt{2 \varsigma} s_{k}}{2}\right) \\
& \xi_{i j k 3}=\tanh \left(\frac{y_{i j}-\boldsymbol{x}_{i j}^{\top} \boldsymbol{\beta}-\sqrt{2 \varsigma} s_{k}}{2}\right),
\end{aligned}
$$

em que $s_{k}$ é o $k$-ésimo zero tabulado do polinômio de Hermite de grau $q$. As derivadas de (B.1), (B.2) e (B.3) em relação a $\gamma=\left[\alpha, \beta_{r}, \varsigma\right]^{\top}$ são dadas por

$$
\begin{aligned}
\frac{\partial \xi_{i j k 1}}{\partial \boldsymbol{\gamma}} & =\left[-\frac{1}{\alpha} \xi_{i j k 1},-\frac{1}{2} \xi_{i j k 2} x_{i j r},-\frac{1}{\sqrt{8 \varsigma}} \xi_{i j k 2} s_{k}\right]^{\top}, \\
\frac{\partial \xi_{i j k 2}}{\partial \gamma} & =\left[-\frac{1}{\alpha} \xi_{i j k 2},-\frac{1}{2} \xi_{i j k 1} x_{i j r},-\frac{1}{\sqrt{8 \varsigma}} \xi_{i j k 1} s_{k}\right]^{\top}, \\
\frac{\partial \xi_{i j k 3}}{\partial \boldsymbol{\gamma}} & =\left[0,-\frac{1}{2} \operatorname{sech}^{2}\left(C_{i j k}\right) x_{i j r},-\frac{1}{\sqrt{8 \varsigma}} \operatorname{sech}^{2}\left(C_{i j k}\right) s_{k}\right]^{\top}, C_{i j k}=\left(\frac{y_{i j}-\boldsymbol{x}_{i j}^{\top} \boldsymbol{\beta}-\sqrt{2 \varsigma} s_{k}}{2}\right)
\end{aligned}
$$

em que,

$$
\begin{aligned}
\frac{\partial C_{i j k}}{\partial \beta_{r}} & =-\frac{1}{2} x_{i j r} \\
\frac{\partial C_{i j k}}{\partial \varsigma} & =-\frac{1}{\sqrt{8 \varsigma}} s_{k} .
\end{aligned}
$$





\section{Apêndice C}

\section{Relações usadas na Matriz Hessiana}

Nesta apêndice são apresentadas as relações usadas nas derivadas da matriz de informação para os modelos log-Birnbaum-Saunders e log-Birnbaum-Saunders-t de intercepto aleatório.

\section{C.1 Relações entre cosh e senh}

Nesta seção apresentamos alguns resultados que serão úteis nos cálculos das derivadas.

$\star$ Baseado em que $2 \cosh (x) \operatorname{senh}(x)=\operatorname{senh}(2 x)$ (Gradshteyn e Ryzhik, 2007, página 35), temos que

$$
\begin{aligned}
\xi_{i j k 1} \xi_{i j k 2} & =\frac{2}{\alpha} \cosh \left(C_{i j k}\right) \frac{2}{\alpha} \operatorname{senh}\left(C_{i j k}\right) \\
& =\frac{4}{\alpha^{2}} \cosh \left(C_{i j k}\right) \operatorname{senh}\left(C_{i j k}\right) \\
& =\frac{2}{\alpha^{2}} \operatorname{senh}\left(2 C_{i j k}\right) .
\end{aligned}
$$

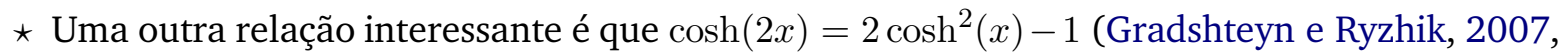
página 35) e $\cosh ^{2}(x)-\operatorname{senh}^{2}(x)=1$ (Gradshteyn e Ryzhik, 2007, página 29).

Finalmente, temos que

$$
\begin{aligned}
\cosh \left(2 C_{i j k}\right) & =2 \cosh ^{2}\left(C_{i j k}\right)-1 \\
& =\cosh ^{2}\left(C_{i j k}\right)+\operatorname{senh}^{2}\left(C_{i j k}\right) .
\end{aligned}
$$





\section{Apêndice D}

\section{Matrices $\Delta$ no Modelo $\log$-BS-t de intercepto aleatório}

Neste apêndice apresentamos as matrices $\Delta$, para a perturbação aditiva da resposta e perturbação aditiva de uma variável explicativa no modelo log-BS-t de intercepto aleatório.

\section{D.1 Perturbação aditiva da resposta}

Vamos perturbar os valores da variável resposta da seguinte forma: $y_{i j}\left(\omega_{i}\right)=y_{i j}+\omega_{i}$, em que $\omega_{i}$ é o $i$-ésimo elemento do vetor $\boldsymbol{\omega}=\left[\omega_{1}, \ldots, \omega_{n}\right]^{\top}$. Se definimos $b_{i 1}^{\star}=\frac{1}{D_{\omega_{i 1}}^{\star}(\boldsymbol{\theta})}, c_{i 1}^{\star}=\frac{\partial D_{\omega_{i 1}}^{\star}(\boldsymbol{\theta})}{\partial \omega_{i}}$, $\boldsymbol{d}_{i 1 \gamma}^{\star}=\frac{\partial D_{\omega_{i 1}}^{\star}(\boldsymbol{\theta})}{\partial \boldsymbol{\gamma}}$, em que

$$
\begin{gathered}
D_{\omega_{i 1}}^{\star}(\boldsymbol{\theta})=\sum_{k=1}^{q} \frac{v_{k}}{\sqrt{\pi}} A_{\omega_{i 1 k}}^{\star}(\boldsymbol{\theta}) B_{\omega_{i 1 k}}^{\star}(\boldsymbol{\theta}), B_{\omega_{i 1 k}}^{\star}(\boldsymbol{\theta})=\exp \left(\sum_{j \in C} \log \left(1-\Phi_{t}\left(\xi_{i j k 2}\left(\omega_{i}\right)\right)\right)\right) \\
A_{\omega_{i 1 k}}^{\star}(\boldsymbol{\theta})=\exp \left(\sum_{j \in O}\left[-\frac{\nu+1}{2} \log \left(1+\frac{\xi_{i j k 2}^{2}\left(\omega_{i}\right)}{\nu}\right)+\log \left(\xi_{i j k 1}\left(\omega_{i}\right)\right)\right]\right), \\
\xi_{i j k 1}\left(\omega_{i}\right)=\frac{2}{\alpha} \cosh \left(\frac{y_{i j}\left(\omega_{i}\right)-\boldsymbol{x}_{i j}^{\top} \boldsymbol{\beta}-\sqrt{2 \varsigma} s_{k}}{2}\right), \quad \xi_{i j k 2}\left(\omega_{i}\right)=\frac{2}{\alpha} \operatorname{senh}\left(\frac{y_{i j}\left(\omega_{i}\right)-\boldsymbol{x}_{i j}^{\top} \boldsymbol{\beta}-\sqrt{2 \varsigma} s_{k}}{2}\right),
\end{gathered}
$$

temos que

$$
\boldsymbol{\Delta}_{i \gamma}=b_{i 1}^{\star}\left(\frac{\partial \boldsymbol{d}_{i 1 \gamma}^{\star}}{\partial \omega_{i}}-b_{i 1}^{\star} c_{i 1}^{\star} \boldsymbol{d}_{i 1 \gamma}^{\star}\right), c_{i 1}^{\star}=\sum_{k=1}^{q} \frac{v_{k}}{\sqrt{\pi}} A_{\omega_{i 1 k}}^{\star}(\boldsymbol{\theta}) B_{\omega_{i 1 k}}^{\star}(\boldsymbol{\theta}) F_{t}\left(y_{i j}\left(\omega_{i}\right)\right),
$$

em que,

$$
\begin{aligned}
& F_{t}\left(y_{i j}\left(\omega_{i}\right)\right)=\frac{1}{2} \sum_{j \in O}\left[\xi_{i j k 3}\left(\omega_{i}\right)-\xi_{i j k 2}\left(\omega_{i}\right) \xi_{i j k 1}\left(\omega_{i}\right) w\left(\xi_{i j k 2}^{2}\left(\omega_{i}\right)\right)\right]-\frac{1}{2} \sum_{j \in C} h_{t}\left(\xi_{i j k 2}\left(\omega_{i}\right)\right) \xi_{i j k 1}\left(\omega_{i}\right), \\
& \xi_{i j k 3}\left(\omega_{i}\right)=\tanh \left(\frac{y_{i j}\left(\omega_{i}\right)-\boldsymbol{x}_{i j}^{\top} \boldsymbol{\beta}-\sqrt{2 \varsigma} s_{k}}{2}\right) .
\end{aligned}
$$


Por outro lado,

$$
\boldsymbol{d}_{i 1 \gamma}^{\star}=\sum_{k=1}^{q} \frac{v_{k}}{\sqrt{\pi}} A_{\omega_{i 1 k}}^{\star}(\boldsymbol{\theta}) B_{\omega_{i 1 k}}^{\star}(\boldsymbol{\theta}) \boldsymbol{F}_{\omega_{i 1 k \gamma}}^{\star}(\boldsymbol{\theta}),
$$

com

$$
\begin{aligned}
\boldsymbol{F}_{\omega_{i 1 k \gamma}}^{\star}(\boldsymbol{\theta})= & \sum_{j \in O}\left[\xi_{i j k 1}^{-1}\left(\omega_{i}\right) \frac{\partial \xi_{i j k 1}\left(\omega_{i}\right)}{\partial \boldsymbol{\gamma}}-\xi_{i j k 2}\left(\omega_{i}\right) w\left(\xi_{i j k 2}^{2}\left(\omega_{i}\right)\right) \frac{\partial \xi_{i j k 2}\left(\omega_{i}\right)}{\partial \boldsymbol{\gamma}}\right] \\
& -\sum_{j \in C} h_{t}\left(\xi_{i j k 2}\left(\omega_{i}\right)\right) \frac{\partial \xi_{i j k 2}\left(\omega_{i}\right)}{\partial \boldsymbol{\gamma}} .
\end{aligned}
$$

Portanto,

$$
\frac{\partial \boldsymbol{d}_{i 1 \gamma}^{\star}}{\partial \omega_{i}}=\sum_{k=1}^{q} \frac{v_{k}}{\sqrt{\pi}} A_{\omega_{i 1 k}}^{\star}(\boldsymbol{\theta}) B_{\omega_{i 1 k}}^{\star}(\boldsymbol{\theta})\left[F_{t}\left(y_{i j}\left(\omega_{i}\right)\right) \boldsymbol{F}_{\omega_{i 1 k \gamma}}^{\star}(\boldsymbol{\theta})+\frac{\partial \boldsymbol{F}_{\omega_{i 1 k \gamma}}^{\star}(\boldsymbol{\theta})}{\partial \omega_{i}}\right],
$$

em que,

$$
\frac{\partial \boldsymbol{F}_{\omega_{i 1 k \gamma}}^{\star}(\boldsymbol{\theta})}{\partial \omega_{i}}=\left[\frac{\partial F_{\omega_{i 1 k \alpha}}^{\star}(\boldsymbol{\theta})}{\partial \omega_{i}}, \frac{\partial F_{\omega_{i 1 k \beta_{r}}}^{\star}(\boldsymbol{\theta})}{\partial \omega_{i}}, \frac{\partial F_{\omega_{i 1 k \varsigma}}^{\star}(\boldsymbol{\theta})}{\partial \omega_{i}}\right]^{\top} .
$$

Para calcular $\boldsymbol{\Delta}_{i \gamma}$ dado na equação (D.1) precisamos determinar $\boldsymbol{d}_{i 1 \gamma}^{\star}$ e $\frac{\partial \boldsymbol{d}_{i 1 \gamma}^{\star}}{\partial \omega_{i}}$ que dependem de $\boldsymbol{F}_{\omega_{i 1 k \gamma}}^{\star}(\boldsymbol{\theta})$ e $\frac{\boldsymbol{F}_{\omega_{i 1 k \gamma}}^{\star}(\boldsymbol{\theta})}{\partial \omega_{i}}$, respectivamente. A seguir apresentamos as expressões com base nas equações (D.2) e (D.3).

$$
\begin{aligned}
F_{\omega_{i 1 k \alpha}}^{\star}(\boldsymbol{\theta}) & =\frac{1}{\alpha} \sum_{j \in O}\left(w\left(\xi_{i j k 2}^{2}\left(\omega_{i}\right)\right) \xi_{i j k 2}^{2}\left(\omega_{i}\right)-1\right)+\frac{1}{\alpha} \sum_{j \in C} h_{t}\left(\xi_{i j k 2}\left(\omega_{i}\right)\right) \xi_{i j k 2}\left(\omega_{i}\right) . \\
\frac{\partial F_{\omega_{i 1 k \alpha}}^{\star}(\boldsymbol{\theta})}{\partial \omega_{i}} & =\frac{1}{2 \alpha}\left[\sum_{j \in O} \xi_{i j k 2}\left(\omega_{i}\right) \xi_{i j k 1}\left(\omega_{i}\right)\left(2 w\left(\xi_{i j k 2}^{2}\left(\omega_{i}\right)\right)+\xi_{i j k 2}\left(\omega_{i}\right) w^{\prime}\left(\xi_{i j k 2}^{2}\left(\omega_{i}\right)\right)\right)\right] \\
& +\frac{1}{2 \alpha}\left[\sum_{j \in C} \xi_{i j k 1}\left(\omega_{i}\right)\left(h_{t}^{\prime}\left(\xi_{i j k 2}\left(\omega_{i}\right)\right) \xi_{i j k 2}\left(\omega_{i}\right)+h_{t}\left(\xi_{i j k 2}\left(\omega_{i}\right)\right)\right)\right],
\end{aligned}
$$

em que

$$
\begin{aligned}
& w^{\prime}\left(\xi_{i j k 2}\left(\omega_{i}\right)\right)=-\frac{2 \xi_{i j k 2}\left(\omega_{i}\right)}{\left(\nu+\xi_{i j k 2}^{2}\left(\omega_{i}\right)\right)} w\left(\xi_{i j k 2}^{2}\left(\omega_{i}\right)\right) \text { e } h_{t}^{\prime}\left(\xi_{i j k 2}\left(\omega_{i}\right)\right)=\frac{\mathrm{d} h_{t}\left(\xi_{i j k 2}\left(\omega_{i}\right)\right)}{\mathrm{d} \xi_{i j k 2}\left(\omega_{i}\right)} . \\
& F_{\omega_{i 1 k \beta_{r}}}^{\star}(\boldsymbol{\theta})=\frac{1}{2} \sum_{j \in O}\left(w\left(\xi_{i j k 2}^{2}\right) \xi_{i j k 1} \xi_{i j k 2}-\xi_{i j k 3}\right) x_{i j r}+\frac{1}{2} \sum_{j \in C} h_{t}\left(\xi_{i j k 2}\right) \xi_{i j k 1} x_{i j r} .
\end{aligned}
$$




$$
\begin{aligned}
\frac{\partial F_{\omega_{i 1 k \beta r}}^{\star}(\boldsymbol{\theta})}{\partial \omega_{i}}= & \frac{1}{4} \sum_{j \in O} \xi_{i j k 1}^{2}\left(\omega_{i}\right) \xi_{i j k 2}\left(\omega_{i}\right) w^{\prime}\left(\xi_{i j k 2}^{2}\left(\omega_{i}\right)\right) x_{i j r} \\
& +\frac{1}{4} \sum_{j \in O}\left(\xi_{i j k 1}^{2}\left(\omega_{i}\right)+\xi_{i j k 2}^{2}\left(\omega_{i}\right)\right) w\left(\xi_{i j k 2}^{2}\left(\omega_{i}\right)\right)-\left(1-\xi_{i j k 3}^{2}\left(\omega_{i}\right)\right) x_{i j r} \\
& -\frac{1}{4} \sum_{j \in C}\left(h_{t}^{\prime}\left(\xi_{i j k 2}\left(\omega_{i}\right)\right) \xi_{i j k 1}^{2}\left(\omega_{i}\right)+h_{t}\left(\xi_{i j k 2}\left(\omega_{i}\right)\right) \xi_{i j k 2}\left(\omega_{i}\right)\right) x_{i j r} . \\
F_{\omega_{i 1 k \varsigma}}^{\star}(\boldsymbol{\theta})= & \frac{1}{\sqrt{8 \varsigma}} \sum_{j \in O}\left(w\left(\xi_{i j k 2}^{2}\right) \xi_{i j k 1} \xi_{i j k 2}-\xi_{i j k 3}\right) s_{k}+\frac{1}{\sqrt{8 \varsigma}} \sum_{j \in C} h_{t}\left(\xi_{i j k 2}\right) \xi_{i j k 1} s_{k} . \\
\frac{\partial F_{\omega_{i 1 k \varsigma}}^{\star}(\boldsymbol{\theta})}{\partial \omega_{i}}= & \frac{1}{\sqrt{32 \varsigma}} \sum_{j \in O} \xi_{i j k 1}^{2}\left(\omega_{i}\right) \xi_{i j k 2}\left(\omega_{i}\right) w^{\prime}\left(\xi_{i j k 2}^{2}\left(\omega_{i}\right)\right) s_{k} \\
& +\frac{1}{\sqrt{32 \varsigma}} \sum_{j \in O}\left(\xi_{i j k 1}^{2}\left(\omega_{i}\right)+\xi_{i j k 2}^{2}\left(\omega_{i}\right)\right) w\left(\xi_{i j k 2}^{2}\left(\omega_{i}\right)\right)-\left(1-\xi_{i j k 3}^{2}\left(\omega_{i}\right)\right) s_{k} \\
& +\frac{1}{\sqrt{32 \varsigma}} \sum_{j \in C}\left(h_{t}^{\prime}\left(\xi_{i j k 2}\left(\omega_{i}\right)\right) \xi_{i j k 1}^{2}\left(\omega_{i}\right)+h_{t}\left(\xi_{i j k 2}\left(\omega_{i}\right)\right) \xi_{i j k 2}\left(\omega_{i}\right)\right) s_{k} .
\end{aligned}
$$

Portanto, a matriz $\boldsymbol{\Delta}_{i \gamma}$ fica dada por

$$
\begin{aligned}
\Delta_{i \alpha} & =b_{i 1}^{\star}\left(\frac{\partial d_{i 1 \alpha}^{\star}}{\partial \omega_{i}}-b_{i 1}^{\star} c_{i 1}^{\star} d_{i 1 \alpha}^{\star}\right), \\
\Delta_{i \beta_{r}} & =b_{i 1}^{\star}\left(\frac{\partial d_{i 1 \beta_{r}}^{\star}}{\partial \omega_{i}}-b_{i 1}^{\star} c_{i 1}^{\star} d_{i 1 \beta_{r}}^{\star}\right), \\
\Delta_{i \varsigma} & =b_{i 1}^{\star}\left(\frac{\partial d_{i 1 \varsigma}^{\star}}{\partial \omega_{i}}-b_{i 1}^{\star} c_{i 1}^{\star} d_{i 1 \varsigma}^{\star}\right),
\end{aligned}
$$

\section{D.2 Perturbação aditiva de uma variável explicativa}

Consideremos a perturbação dos valores da $p$-ésima variável explicativa, assumida contínua, como $x_{i j p}\left(\omega_{i}\right)=x_{i j p}+\omega_{i}$, em que $\omega_{i}$ representa o $i$-ésimo elemento do vetor $\boldsymbol{\omega}=\left[\omega_{1}, \ldots, \omega_{n}\right]^{\top}$ que denota o vetor de perturbações do modelo. Se definimos $b_{i 2}^{\star}=\frac{1}{D_{\omega_{i 2}^{\star}}(\boldsymbol{\theta})}, c_{i 2}^{\star}=\frac{\partial D_{\omega_{i 2}}^{\star}(\boldsymbol{\theta})}{\partial \omega_{i}}$ e $\boldsymbol{d}_{i 2 \gamma}^{\star}=$ $\frac{\partial D_{\omega_{i 2}}^{\star}(\boldsymbol{\theta})}{\partial \gamma}$, em que

$$
\begin{aligned}
D_{\omega_{i 2}}^{\star}(\boldsymbol{\theta}) & =\sum_{k=1}^{q} \frac{v_{k}}{\sqrt{\pi}} A_{\omega_{i 2 k}}^{\star}(\boldsymbol{\theta}) B_{\omega_{i 2 k}}^{\star}(\boldsymbol{\theta}), B_{\omega_{i 2 k}}^{\star}(\boldsymbol{\theta})=\exp \left(\sum_{j \in C} \log \left(1-\Phi\left(\xi_{i j k 2}\left(\omega_{i}\right)\right)\right)\right), \\
A_{\omega_{i 2 k}}^{\star}(\boldsymbol{\theta}) & =\exp \left(\sum_{j \in O}\left[-\frac{\nu+1}{2} \log \left(1+\frac{\xi_{i j k 2}^{2}\left(\omega_{i}\right)}{\nu}\right)+\log \left(\xi_{i j k 1}\left(\omega_{i}\right)\right)\right]\right) \mathrm{e}
\end{aligned}
$$

$\xi_{i j k 1}\left(\omega_{i}\right)=\frac{2}{\alpha} \cosh \left(\frac{y_{i j}-\boldsymbol{x}_{i j}^{\top}\left(\omega_{i}\right) \boldsymbol{\beta}-\sqrt{2 \varsigma} s_{k}}{2}\right), \quad \xi_{i j k 2}\left(\omega_{i}\right)=\frac{2}{\alpha} \operatorname{senh}\left(\frac{y_{i j}-\boldsymbol{x}_{i j}^{\top}\left(\omega_{i}\right) \boldsymbol{\beta}-\sqrt{2 \varsigma} s_{k}}{2}\right)$. 
$\operatorname{com} \boldsymbol{x}_{i j}^{\top}\left(\omega_{i}\right) \boldsymbol{\beta}=x_{i j 1} \beta_{1}+\ldots+x_{i j, p-1} \beta_{p-1}+x_{i j p}\left(\omega_{i}\right) \beta_{p}$, temos que

$$
\boldsymbol{\Delta}_{i \gamma}=b_{i 2}^{\star}\left(\frac{\partial \boldsymbol{d}_{i 2 \gamma}^{\star}}{\partial \omega_{i}}-b_{i 2}^{\star} c_{i 2}^{\star} \boldsymbol{d}_{i 2 \gamma}^{\star}\right), \quad c_{i 2}^{\star}=\sum_{k=1}^{q} \frac{v_{k}}{\sqrt{\pi}} A_{\omega_{i 2 k}}^{\star}(\boldsymbol{\theta}) B_{\omega_{i 2 k}}^{\star}(\boldsymbol{\theta}) F_{t}\left(x_{i j p}\left(\omega_{i}\right)\right)
$$

em que,

$$
\begin{aligned}
F_{t}\left(x_{i j p}\left(\omega_{i}\right)\right) & =-\frac{\beta_{p}}{2} \sum_{j \in O}\left[\xi_{i j k 3}\left(\omega_{i}\right)-\xi_{i j k 2}\left(\omega_{i}\right) \xi_{i j k 1}\left(\omega_{i}\right) w\left(\xi_{i j k 2}^{2}\left(\omega_{i}\right)\right)\right]+\frac{\beta_{p}}{2} \sum_{j \in C} h_{t}\left(\xi_{i j k 2}\left(\omega_{i}\right)\right) \xi_{i j k 1}\left(\omega_{i}\right), \\
\xi_{i j k 3}\left(\omega_{i}\right) & =\tanh \left(\frac{y_{i j}-\boldsymbol{x}_{i j}^{\top}\left(\omega_{i}\right) \boldsymbol{\beta}-\sqrt{2 \varsigma} s_{k}}{2}\right) .
\end{aligned}
$$

Por outro lado,

$$
\boldsymbol{d}_{i 2 \gamma}^{\star}=\sum_{k=1}^{q} \frac{v_{k}}{\sqrt{\pi}} A_{\omega_{i 2 k}}^{\star}(\boldsymbol{\theta}) B_{\omega_{i 2 k}}^{\star}(\boldsymbol{\theta}) \boldsymbol{F}_{\omega_{i 2 k \gamma}}^{\star}(\boldsymbol{\theta})
$$

com

$$
\begin{aligned}
\boldsymbol{F}_{\omega_{i 2 k \gamma}}^{\star}(\boldsymbol{\theta}) & =\sum_{j \in O}\left[\xi_{i j k 1}^{-1}\left(\omega_{i}\right) \frac{\partial \xi_{i j k 1}\left(\omega_{i}\right)}{\partial \boldsymbol{\gamma}}-\xi_{i j k 2}\left(\omega_{i}\right) w\left(\xi_{i j k 2}^{2}\left(\omega_{i}\right)\right) \frac{\partial \xi_{i j k 2}\left(\omega_{i}\right)}{\partial \boldsymbol{\gamma}}\right] \\
& -\sum_{j \in C} h_{t}\left(\xi_{i j k 2}\left(\omega_{i}\right)\right) \frac{\partial \xi_{i j k 2}\left(\omega_{i}\right)}{\partial \boldsymbol{\gamma}}
\end{aligned}
$$

Portanto,

$$
\frac{\partial \boldsymbol{d}_{i 2 \gamma}^{\star}}{\partial \omega_{i}}=\sum_{k=1}^{q} \frac{v_{k}}{\sqrt{\pi}} A_{\omega_{i 2 k}}^{\star}(\boldsymbol{\theta}) B_{\omega_{i 2 k}}^{\star}(\boldsymbol{\theta})\left[F_{t}\left(x_{i j p}\left(\omega_{i}\right)\right) \boldsymbol{F}_{\omega_{i 2 k \gamma}}^{\star}(\boldsymbol{\theta})+\frac{\partial \boldsymbol{F}_{\omega_{i 2 k \gamma}}^{\star}(\boldsymbol{\theta})}{\partial \omega_{i}}\right],
$$

em que,

$$
\frac{\partial \boldsymbol{F}_{\omega_{i 2 k \gamma}}^{\star}(\boldsymbol{\theta})}{\partial \omega_{i}}=\left[\frac{\partial F_{\omega_{i 2 k \alpha}}^{\star}(\boldsymbol{\theta})}{\partial \omega_{i}}, \frac{\partial F_{\omega_{i 2 k \beta_{r}}}^{\star}(\boldsymbol{\theta})}{\partial \omega_{i}}, \frac{\partial F_{\omega_{i 2 k \varsigma}}^{\star}(\boldsymbol{\theta})}{\partial \omega_{i}}\right]^{\top}
$$

Para calcular $\boldsymbol{\Delta}_{i \gamma}$ dado na equação (D.4) precisamos determinar $\boldsymbol{d}_{i 2 \gamma}^{\star}$ e $\frac{\partial \boldsymbol{d}_{i 2 \gamma}^{\star}}{\partial \omega_{i}}$ que dependem de $\boldsymbol{F}_{\omega_{i 2 k \gamma}}^{\star}(\boldsymbol{\theta})$ e $\frac{\boldsymbol{F}_{\omega_{i 2 k \gamma}}^{\star}(\boldsymbol{\theta})}{\partial \omega_{i}}$, respectivamente. A seguir apresentamos as expressões com base nas equações (D.5) e (D.6).

$$
\begin{aligned}
F_{\omega_{i 2 k \alpha}}^{\star}(\boldsymbol{\theta})= & \frac{1}{\alpha} \sum_{j \in O}\left(w\left(\xi_{i j k 2}^{2}\left(\omega_{i}\right)\right) \xi_{i j k 2}^{2}\left(\omega_{i}\right)-1\right)+\frac{1}{\alpha} \sum_{j \in C} h_{t}\left(\xi_{i j k 2}\left(\omega_{i}\right)\right) \xi_{i j k 2}\left(\omega_{i}\right) \\
\frac{\partial F_{\omega_{i 2 k \alpha}}^{\star}(\boldsymbol{\theta})}{\partial \omega_{i}}= & -\frac{\beta_{p}}{2 \alpha} \sum_{j \in O}\left[\xi_{i j k 2}\left(\omega_{i}\right) \xi_{i j k 1}\left(\omega_{i}\right)\left(2 w\left(\xi_{i j k 2}^{2}\left(\omega_{i}\right)\right)+\xi_{i j k 2}\left(\omega_{i}\right) w^{\prime}\left(\xi_{i j k 2}^{2}\left(\omega_{i}\right)\right)\right]\right. \\
& -\frac{\beta_{p}}{2 \alpha} \sum_{j \in C}\left[\xi_{i j k 1}\left(\omega_{i}\right)\left(h_{t}^{\prime}\left(\xi_{i j k 2}\left(\omega_{i}\right)\right) \xi_{i j k 2}\left(\omega_{i}\right)+h_{t}\left(\xi_{i j k 2}\left(\omega_{i}\right)\right)\right)\right]
\end{aligned}
$$


em que

$$
\begin{aligned}
w^{\prime}\left(\xi_{i j k 2}\left(\omega_{i}\right)\right) & =\frac{\mathrm{d} w\left(\xi_{i j k 2}\left(\omega_{i}\right)\right)}{\mathrm{d} \xi_{i j k 2}\left(\omega_{i}\right)} \text { e } \quad h_{t}^{\prime}\left(\xi_{i j k 2}\left(\omega_{i}\right)\right)=\frac{\mathrm{d} h_{t}\left(\xi_{i j k 2}\left(\omega_{i}\right)\right)}{\mathrm{d} \xi_{i j k 2}\left(\omega_{i}\right)} . \\
F_{\omega_{i 2 k \beta_{r}}}^{\star}(\boldsymbol{\theta}) & =\frac{1}{2} \sum_{j \in O}\left(w\left(\xi_{i j k 2}^{2}\right) \xi_{i j k 1} \xi_{i j k 2}-\xi_{i j k 3}\right) x_{i j r}+\frac{1}{2} \sum_{j \in C} h_{t}\left(\xi_{i j k 2}\right) \xi_{i j k 1} x_{i j r} . \\
\frac{\partial F_{\omega_{i 2 k \beta_{r}}}^{\star}(\boldsymbol{\theta})}{\partial \omega_{i}}= & -\frac{\beta_{p}}{4} \sum_{j \in O}\left(\xi_{i j k 1}^{2}\left(\omega_{i}\right) \xi_{i j k 2}\left(\omega_{i}\right) w^{\prime}\left(\xi_{i j k 2}^{2}\left(\omega_{i}\right)\right)+\left(\xi_{i j k 1}^{2}\left(\omega_{i}\right)+\xi_{i j k 2}^{2}\left(\omega_{i}\right)\right) w\left(\xi_{i j k 2}^{2}\left(\omega_{i}\right)\right)\right) \\
& +\frac{\beta_{p}}{4}\left[\sum_{j \in O}\left(1-\xi_{i j k 3}^{2}\left(\omega_{i}\right)\right)-\sum_{j \in C}\left(h_{t}^{\prime}\left(\xi_{i j k 2}\left(\omega_{i}\right)\right) \xi_{i j k 1}^{2}\left(\omega_{i}\right)+h_{t}\left(\xi_{i j k 2}\left(\omega_{i}\right)\right) \xi_{i j k 2}\left(\omega_{i}\right)\right)\right] . \\
F_{\omega_{i 2 k \varsigma}}^{\star}(\boldsymbol{\theta}) & =\frac{1}{\sqrt{8 \varsigma}} \sum_{j \in O}\left(w\left(\xi_{i j k 2}^{2}\right) \xi_{i j k 1} \xi_{i j k 2}-\xi_{i j k 3}\right) s_{k}+\frac{1}{\sqrt{8 \varsigma}} \sum_{j \in C} h_{t}\left(\xi_{i j k 2}\right) \xi_{i j k 1} s_{k} . \\
\frac{\partial F_{\omega_{i 2 k \varsigma}}(\boldsymbol{\theta})}{\partial \omega_{i}}= & -\frac{\beta_{p}}{\sqrt{32 \varsigma}} \sum_{j \in O}\left(\xi_{i j k 1}^{2}\left(\omega_{i}\right) \xi_{i j k 2}\left(\omega_{i}\right) w^{\prime}\left(\xi_{i j k 2}^{2}\left(\omega_{i}\right)\right)+\left(\xi_{i j k 1}^{2}\left(\omega_{i}\right)+\xi_{i j k 2}^{2}\left(\omega_{i}\right)\right) w\left(\xi_{i j k 2}^{2}\left(\omega_{i}\right)\right)\right) \\
+ & \frac{\beta_{p}}{\sqrt{32 \varsigma}}\left[\sum_{j \in O}\left(1-\xi_{i j k 3}^{2}\left(\omega_{i}\right)\right)-\sum_{j \in C}\left(h_{t}^{\prime}\left(\xi_{i j k 2}\left(\omega_{i}\right)\right) \xi_{i j k 1}^{2}\left(\omega_{i}\right)+h_{t}\left(\xi_{i j k 2}\left(\omega_{i}\right)\right) \xi_{i j k 2}\left(\omega_{i}\right)\right)\right] .
\end{aligned}
$$

Portanto, a matriz $\boldsymbol{\Delta}_{i \gamma}$ fica dada por

$$
\begin{aligned}
\Delta_{i \alpha} & =b_{i 2}^{\star}\left(\frac{\partial d_{i 2 \alpha}^{\star}}{\partial \omega_{i}}-b_{i 2}^{\star} c_{i 2}^{\star} d_{i 2 \alpha}^{\star}\right), \\
\Delta_{i \beta_{r}} & =b_{i 2}^{\star}\left(\frac{\partial d_{i 2 \beta_{r}}^{\star}}{\partial \omega_{i}}-b_{i 2}^{\star} c_{i 2}^{\star} d_{i 2 \beta_{r}}^{\star}\right), \\
\Delta_{i \varsigma} & =b_{i 2}^{\star}\left(\frac{\partial d_{i 2 \varsigma}^{\star}}{\partial \omega_{i}}-b_{i 2}^{\star} c_{i 2}^{\star} d_{i 2 \varsigma}^{\star}\right),
\end{aligned}
$$





\section{Apêndice E}

\section{Código SAS para os Modelos log-BS e log-BS-t de Intercepto Aleatório}

A seguir apresentamos o código SAS usado para a estimação dos parâmetros dos modelos logBirnbaum-Saunders e log-Birnbaum-Saunders-t de intercepto aleatório.

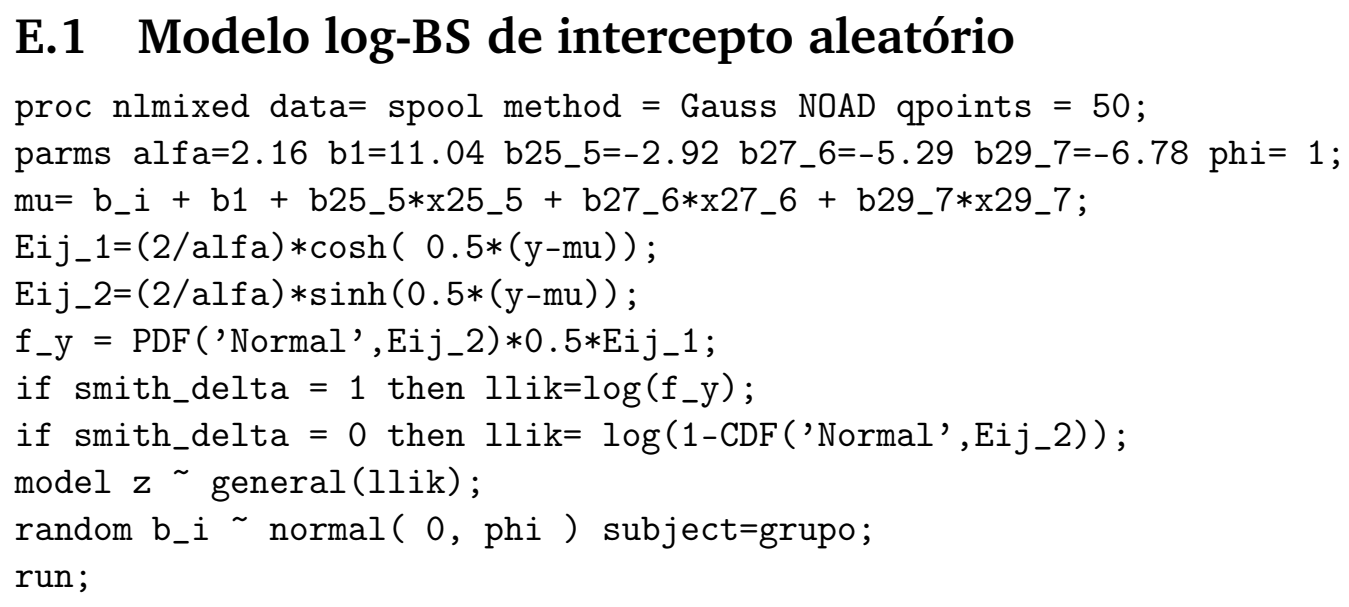

\section{E.2 Modelo log-BS-t de intercepto aleatório}

proc nlmixed data $=$ spool method $=$ Gauss NOAD qpoints $=50$;

parms alfa=2.16 b1=11.04 b25_5=-2.92 b27_6=-5.29 b29_7=-6.78 phi=1;

$\mathrm{mu}=\mathrm{b}_{-} i+\mathrm{b} 1+\mathrm{b} 25 \_5 * \mathrm{x} 25 \_5+\mathrm{b} 27 \_6 * \mathrm{x} 27 \_6+\mathrm{b} 29 \_7 * \mathrm{x} 29 \_7$;

Eij_1= (2/alfa $) * \cosh (0.5 *(y-m u))$;

Eij_2= (2/alfa $) * \sinh (0.5 *(y-m u))$;

$f_{-} y=\operatorname{PDF}(' T$ ', Eij_2, 10)*0.5*Eij_1;

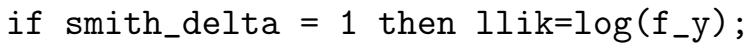

if $\operatorname{smith}_{\text {delta }}=0$ then $l \operatorname{lik}=\log \left(1-\operatorname{CDF}\left({ }^{\prime} \mathrm{T}\right.\right.$ ', Eij_2,10));

model $z$ oeneral(lik);

random b_i1 normal (0,phi_1) subject=grupo;

run; 



\section{Referências Bibliográficas}

Abramowitz e Stegun(1972) M. Abramowitz e I. Stegun. Handbook of Mathematical Functions: with Formulas, Graphs, and Mathematical Tables. National Bureau of Standards, Washington D.C.

Achcar e Espinosa(1991) J. A. Achcar e M. M. Espinosa. Bayesian method in accelerated life tests considering a log-linear model for the Birnbaum-Saunders distribution. Revista Brasileira de Estatística, 52:47-68.

Anderson e Fang(1987) T. W. Anderson e K. T. Fang. Cochran's theorem for elliptically contoured distributions. Sankhya Serie A, 49:305-315.

Andrews e Mallows(1974) D. F. Andrews e C. L. Mallows. Scale mixtures of normal distributions. Journal of the Royal Statistical Society Series B, 36:99-102.

Balakrishnan et al.(2009) N. Balakrishnan, V. Leiva, A. Sanhueza, e F. Vilca. Estimation in the Birnbaum-Saunders distribution based on scale-mixture of normals and the EM-algorithm. Statistics and Operations Research Transactions, 33:171-192.

Barros(2007) M. Barros. Modelos de Regressão Birnbaum-Saunders Generalizados. Tese de Doutorado IME-USP, São Paulo (Brasil), 2007.

Barros et al.(2008) M. Barros, G. A. Paula, e V. Leiva. A new class of survival regression models with heavy-tailed errors: robustness and diagnostics. Lifetime Data Analysis, 14:316-332.

Barros et al.(2009) M. Barros, G. A. Paula, e V. Leiva. An R implementation for Generalized Birnbaum-Saunders distributions. Computational Statistics \& Data Analysis, 53:1511-1528.

Belsley et al.(1980) D. A. Belsley, E. Kuh, e R. E. Welch. Regression Diagnostics, Identifying Influential Data and Sources of Collinearity. New York, Wiley.

Billor e Loynes(1993) N. Billor e R. Loynes. Local influence: a new approach. Communication in Statistics: Theory and Methods, 22:1595-1611.

Birnbaum e Saunders(1969a) Z. W. Birnbaum e S. C. Saunders. A new family of life distributions. Journal of Applied Probability, 6:319-327.

Birnbaum e Saunders(1969b) Z. W. Birnbaum e S. C. Saunders. Estimation for a family of life distributions with applications to fatigue. Journal of Applied Probability, 6:328-347.

Broyden(1970) C. G. Broyden. The Convergence of a Class of Double-rank Minimization Algorithms: 2. The New Algorithm. Journal of the Institute of Mathematics and its Applications, 6: $222-231$. 
Cambanis et al.(1981) S. Cambanis, S. Huang, e G. Simons. On the theory of elliptically contoured distributions. Journal of Multivariate Analysis, 11:365-385.

Casella e Berger(2002) G. Casella e R. Berger. Statistical Inference. Pacific Grove, CA: Duxbury/Thomson Learning.

Chatterjee e Hadi(1988) S. Chatterjee e A. S. Hadi. Sensitivity Analysis in Linear Regression. New York, Wiley.

Colosimo e Giolo(2006) E. A. Colosimo e S. Giolo. Análise de Sobrevivência Aplicada. São Paulo: Edgard Blücher.

Cook(1986) R. D. Cook. Assessment of local influence (with discussion). Journal of the Royal Statistical Society Series B, 48:133-169.

Cook(1987) R. D. Cook. Influence assessment. Journal of Applied Statistics, 14:117-131.

Cook e Weisberg(1982) R. D. Cook e S. Weisberg. Residuals and Influence in Regression. London, Chapman \& Hall.

Cordeiro et al.(2000) G. M. Cordeiro, S. L. P. Ferrari, M. A. Uribe-Opazo, e K. L. P. Vasconcellos. Corrected maximum-likelihood estimation in a class of symmetric nonlinear regression models. Statistics \& Probability, 46:317-328.

Cuminato(2009) J. A. Cuminato. Cálculo Numérico. Instituto de Ciências Matemáticas e de Computação-USP, 2009.

Davis e Rabinowitz(1975) P. Davis e P. Rabinowitz. Methods of Numerical Integration. New York, Academic Press.

Dawid(1977) A. P. Dawid. Spherical matrix distributions and a multivariate model. Journal of the Royal Statistical Society Series B, 39:254-261.

Díaz-García e Domínguez-Molina(2006) J. A. Díaz-García e J. R. Domínguez-Molina. Some generalisations of Birnbaum-Saunders and sinh-normal distributions. International Mathematical Forum, 1:1709-1727.

Díaz-García e Leiva(2005) J. A. Díaz-García e V. Leiva. A new family of life distributions based on the contoured elliptically distributions. Journal of Statistical Planning and Inference, 128: 88-95.

Díaz-García e Leiva(2007) J. A. Díaz-García e V. Leiva. Erratum to a new family of life distributions based on the elliptically contoured distributions: [Journal of Statistical Planning and Inference 128 (2005) 445-457]. Journal of Statistical Planning and Inference, 137:1512-1513.

Dempster et al.(1977) A. P. Dempster, N. M. Laird, e D. B. Rubin. Maximun likelihood from incomplete data via the EM algorithm (with discussion). Journal of the Royal Statistical Society Series B, 39:1-38.

Desmond(1985) A. Desmond. Stochastic models of failure in random environments. Canadian Journal of Statistics, 13:171-183.

Desmond(1986) A. Desmond. On the relationship between two fatigue-life models. IEEE Transactions on Reliability, 35:167-169. 
Diggle et al.(1994) P. J. Diggle, K. Y. Liang, e S. L. Zeger. Analysis of Longitudinal Data. Oxford, Clarendon Press.

Dupuis e Mills(1998) D. J. Dupuis e J. E. Mills. Robust estimation of the Birnbaum-Saunders distribution. IEEE Transactions on Reliability, 47:88-95.

Engelhardt et al.(1981) M. Engelhardt, L. J. Bain, e F. T. Wright. Inferences on the parameters of the Birnbaum-Saunders fatigue life distribution based on maximum likelihood estimation. Technometrics, 23:251-255.

Fahrmeir e Tutz(1994) L. Fahrmeir e G. Tutz. Multivariate Statistical Modelling Based on Generalized Linear Models. New York, Springer.

Fang et al.(1990) K. T. Fang, S. Kotz, e K. W. Ng. Symmetric Multivariate and Related Distributions. London, Chapman \& Hall.

Fernandez e Steel(1999) C. Fernandez e M. Steel. Multivariate Student-t regression models: pitfalls and inference. Biometrika, 86:153-167.

Ferrari e Uribe-Opazo(2001) S. L. P. Ferrari e M. A. Uribe-Opazo. Corrected likelihood ratio tests in class of symmetric linear regression models. Brazilian Journal of Probability and Statistics, 15:49-67.

Fletcher(1970) R. Fletcher. A new approach to variable metric algorithms. The Computer Journal, $13: 317-322$.

Galea et al.(2004) M. Galea, V. Leiva, e G. A. Paula. Influence diagnostics in log-BirnbaumSaunders regression models. Journal of Applied Statistics, 31:1049-1064.

Giampaoli e Singer(2009) V. Giampaoli e J. M. Singer. Likelihood ratio tests for variance components in linear mixed models. Journal of Statistical Planning and Inference, 139:1435 - 1448.

Goldfarb(1970) D. Goldfarb. A family of variable-metric methods derived by variational means. Mathematics of Computation, 24:23-26.

Gradshteyn e Ryzhik(2007) I. S. Gradshteyn e I. M. Ryzhik. Table of integrals, Series, and Products. New York, Academic Press.

Guiraud et al.(2009) P. Guiraud, V. Leiva, e R. Fierro. A non-central version of the BirnbaumSaunders distribution for reliability analysis. IEEE Transactions on Reliability, 58:152-160.

Henderson(1984) C. R. Henderson. Applications of Linear Models in Animal Breeding. University of Guelph Press, Guelph.

Jamshidian(1999) M. Jamshidian. Adaptive robust regression by using a nonlinear regression program. Journal of Statistical Software, 4:1-25.

Johnson et al.(1994) N. L. Johnson, S. Kotz, e N. Balakrishnan. Continuous Univariate Distributions, Vol. 1. New York, Wiley.

Johnson et al.(1995) N. L. Johnson, S. Kotz, e N. Balakrishnan. Continuous Univariate Distributions, Vol. 2. New York, Wiley. 
Kelker(1970) D. Kelker. Distribution theory of spherical distributions and a location scale parameter generalization. Sankhya Serie A, 32:419-430.

Klein e Moeschberger(1997) J. P. Klein e M. L. Moeschberger. Survival Analysis: Techniques for Censored and Truncated Data. New York, Springer.

Laird e Ware(1982) N.M. Laird e J.H. Ware. Random effects models for longitudinal data. Biometrics, 38:963-974.

Lange(2004) K. L. Lange. Optimization. New York, Springer.

Lange e Sinsheimer(1993) K. L. Lange e J. S. Sinsheimer. Normal/independent distributions and their applications in robust regression. Journal of Computational and Graphical Statistics, 2:175-198.

Lange et al.(1989) K. L. Lange, R. J. A. Little, e J. M. G. Taylor. Robust statistical modeling using the t-distribution. Journal of the American Statistical Association, 84(408):881-896.

Laurent e Cook(1992) R. T. ST. Laurent e R. D. Cook. Leverage, local influence and curvature in nonlinear regression. Biometrika, 80(1):99-106.

Lawless(1982) J. F. Lawless. Statistical Models and Methods for Lifetime Data. New York, Wiley.

Leiva et al.(2006) V. Leiva, H. Hernández, e M. Riquelme. A new package for the BirnbaumSaunders distribution. $R$ Journal, 6:35-40.

Leiva et al.(2007) V. Leiva, M. Barros, G. A. Paula, e M. Galea. Influence diagnostics in logBirnbaum-Saunders regression models with censored data. Computational Statistics \& Data Analysis, 51:5694-5707.

Leiva et al.(2008a) V. Leiva, M. Barros, G. A. Paula, e A. Sanhueza. Generalized BirnbaumSaunders Distribution applied to air pollutant concentration. Environmetrics, 19:235-249.

Leiva et al.(2009) V. Leiva, M. Barros, e G. A. Paula. Generalized Birnbaum-Saunders Models using R. Associação Brasileira de Estatística, São Paulo (Brasil).

Leiva et al.(2008b) V. Leiva, M. Riquelme, N. Balakrishnan, e A. Sanhueza. Lifetime analysis based on the generalized birnbaum-saunders distribution. Computational Statistics \& Data Analysis, 52:2079-2097.

Leonard et al.(1989) T. Leonard, J. S. J. Hsu, e K. W. Tsui. Bayesian marginal inference. Journal of the American Statistical Association, 84:1051-1058.

Lesaffre e Spiessens(2001) E. Lesaffre e B. Spiessens. On the effect of the number of quadrature points in a logistic random-effects model: an example. Applied Statistics, 50(3):325-335.

Lesaffre e Verbeke(1998) E. Lesaffre e G. Verbeke. Local influence in linear mixed models. Biometrics, 54:570-582.

Littell et al.(1996) R. C. Littell, G. A. Milliken, W. W. Stroup, e R. D. Wolfinger. SAS System of Mixed Models. Cary, North Carolina: SAS Institute.

Liu e Pierce(1994) Q. Liu e D. A. Pierce. A note on Gauss-Hermite quadrature. Biometrika, 81: 624-629. 
Lu e Chang(1997) M. Lu e D.S. Chang. Bootstrap prediction intervals for the Birnbaum-Saunders distribution. Microelectronics and Reliability, 37:1213-1216.

McCarter(1999) K. S. McCarter. Estimation and Prediction for the Birnbaum-Saunders Distribution Using Type-II Censored Samples, with a Comparison to the Inverse Gaussian Distribution. Kansas State University, Department of Statistics, College of Arts and Sciences, 1999.

McCulloch e Searle(2001) C. McCulloch e S. Searle. Generalized, Linear and Mixed Models. New York, Wiley.

Montgomery et al.(2001) D. C. Montgomery, E. A. Peck, e G. G. Vining. Introduction to Linear Regression Analysis. New York, Wiley.

Ortega(2001) E. M. M. Ortega. Análise de Influência e Resíduos em Modelos de Regressão Log-Gama Generalizados. Tese de Doutorado IME-USP, São Paulo (Brasil), 2001.

Ortega et al.(2003) E. M. M. Ortega, H. Bolfarine, e G. A. Paula. Influence diagnostic in generalized log-gamma regression models. Computational Statistics \& Data Analysis, 42:165-186.

Ortega et al.(2008) E. M. M. Ortega, G. A. Paula, e H. Bolfarine. Deviance residuals in generalized log-gamma regression models with censored observations. Journal of Statistical Computation and Simulation, 78:747-764.

Ortega et al.(2009) E. M. M. Ortega, V. G. Cancho, e G. A. Paula. Generalized log-gamma regression models with cure fraction. Lifetime Data Analysis, 15:79-106.

Osorio(2006) F. Osorio. Diagnóstico de Influência em Modelos Elípticos com Efeitos Mistos. Tese de Doutorado IME-USP, São Paulo (Brasil), 2006.

Osorio et al.(2007) F. Osorio, G. A. Paula, e M. Galea. Assessment local influence in elliptical linear models with longitudinal structure. Computational Statistics \& Data Analysis, 51:43544368.

Owen e Padgett(1999) W. J. Owen e W.J. Padgett. Accelerated test models for system strength based on Birnbaum-Saunders distribution. Lifetime Data Analysis, 5:133-147.

Pinheiro e Bates(2000) J. C. Pinheiro e D. M. Bates. Mixed-Effects Models in S and S-Plus. New York, Springer.

Pinheiro e Bates(1995) J. C. Pinheiro e D. M. Bates. Approximations to the log-likelihood function in the nonlinear mixed-effects model. Journal of Computational and Graphical Statistics, 4: 12-35.

Pinheiro e Chao(2006) J. C. Pinheiro e E. C. Chao. Efficient laplacian and adaptive gaussian quadrature algorithms for multilevel generalized linear mixed models. Journal of Computational and Graphical Statistics, 15:58-81.

Pinheiro et al.(2001) J. C. Pinheiro, C. Liu, e Y. N. Wu. Efficient algorithms for robust estimation in linear mixed-effects models using the multivariate $t$ distribution. Journal of Computational and Graphical Statistics, 10:249-276.

Poon e Poon(1999) W. Y. Poon e Y. S. Poon. Conformal normal curvature and assessment of local influence. Journal of the Royal Statistical Society Series B, 61:51-61. 
R Development Core Team(2008) R Development Core Team. $R$ : A Language and Environment for Statistical Computing. R Foundation for Statistical Computing, Vienna, Austria. http://www.rproject.org, 2008.

Ravishanker e Dipak(2002) N. Ravishanker e K. Dipak. A First Course in Linear Model Theory. Boca Raton: Chapman \& Hall/CRC.

Rieck(1999) J. R. Rieck. A moment-generating function with application to the BirnbaumSaunders distribution. Communications in Statistics: Theory and Methods, 28:2213-2222.

Rieck e Nedelman(1991) J. R. Rieck e J. R. Nedelman. A log-linear model for the BirnbaumSaunders distribution. Technometrics, 33:51-60.

Russo(2010) C. M. Russo. Modelos Não Lineares Elípticos para Dados Correlacionados. Tese de Doutorado IME-USP, São Paulo (Brasil), 2010.

Sanhueza et al.(2008) A. Sanhueza, V. Leiva, e N. Balakrishnan. The Generalized BirnbaumSaunders distribution and its theory, methodology, and application. Communications in Statistics: Theory and Methods, 37:645-670.

SAS Institute Inc.(2004) SAS Institute Inc. SAS/STAT User's Guide, Version 9.1. Cary, NC: SAS Institute Inc., 2004.

Saunders(1974) S. C. Saunders. A family of random variables closed under reciprocation. Journal of the American Statistical Association, 69:533-539.

Savalli et al.(2006) C. Savalli, G. A. Paula, e F. J. A. Cysneiros. Assessment of variance components in elliptical linear mixed models. Statistical Modelling, 6:59-76.

Schervish(1995) M. J. Schervish. Theory of Statistics. New York, Springer.

Searle et al.(1992) S. R. Searle, G. Casella, e C. E. McCulloch. Variance Components. New York, Wiley.

Self e Liang(1987) S. G. Self e K. Y. Liang. Asymptotic properties of maximum likelihood estimators and likelihood ratio tests under non-standard conditions. Journal of the American Statistical Association, 82:605-610.

Shanno(1970) D. F. Shanno. Conditioning of quasi-newton methods for function minimization. Mathematics of Computation, 24:647-657.

Silvapulle e Silvapulle(1995) M. J. Silvapulle e P. Silvapulle. A score test against one-sided alternatives. Journal of the American Statistical Association, 90:342-349.

Smith(1991) R. L. Smith. Weibull regression models for reliability data. Reliability Engineering \& System Safety, 34:55-77.

Stram e Lee(1994) D. O. Stram e J. W. Lee. Variance components testing in the longitudinal mixed effects model. Biometrics, 50:1171-1177.

Tang et al.(2006) N. Tang, B. Wei, e W. Zhang. Influence diagnostics in nonlinear reproductive dispersion mixed models. Statistics, 40:227-246. 
Tanner(1996) M. A. Tanner. Tools for statistical inference methods for the exploration of posterior distributions and likelihood functions. New York, Springer.

Taylor e Verbyla(2004) J. Taylor e A. Verbyla. Joint modeling of location and scale parameters of t distribution. Statistical Modelling, 4:91-112.

Therneau et al.(1990) T. M. Therneau, P. M. Grambsch, e T. R. Fleming. Martingale-based residuals for survival models. Biometrika, 77:147-160.

Tierney e Kadane(1986) L. Tierney e J. B. Kadane. Accurate approximation for posterior moments and marginal densities. Journal of the American Statistical Association, 81:82-86.

Verbeke e Molenberghs(2000) G. Verbeke e G. Molenberghs. Linear Mixed Models for Longitudinal Data. New York, Springer.

Verbeke e Molenberghs(2003) G. Verbeke e G. Molenberghs. The use of score tests for inference on variance components. Biometrics, 59:254-262.

Vilca-Labra e Leiva(2006) F. Vilca-Labra e V. Leiva. A new fatigue life model based on the family of skew-elliptical distributions. Communications in Statistics: Theory and Methods, 35:1-16.

Vonesh(1992) E. F. Vonesh. Nonlinear models for the analysis of longitudinal data. Statistics in Medicine, 11:1929-1954.

Vonesh(1996) E. F. Vonesh. A note on the use of Laplace's approximation for nonlinear mixedeffects models. Biometrika, 83:447-452.

Vonesh e Chinchilli(1997) E. F. Vonesh e V. M. Chinchilli. Linear and Nonlinear Models for the Analysis of Repeated Measurements. New York, Marcel Dekker.

Vu e Zhou(1997) H. T. V. Vu e S. Zhou. Generalization of likelihood ratio tests under nonstandard conditions. Annals of Statistics, 25:897-916.

Wei et al.(1998) B. C. Wei, Y.Q. Hu, e W.K. Fung. Generalized leverage and its applications. Scandinavian Journal of Statistics, 25:25-37.

Weisberg(2005) S. Weisberg. Applied Linear Regression. New Yersey, Wiley.

Wolfinger(1993) R. Wolfinger. Laplace's approximation for nonlinear mixed models. Biometrika, 80:791-795.

Wolfinger e Lin(1997) R. D. Wolfinger e X. Lin. Two taylor-series approximation methods for nonlinear mixed models. Computational Statistics and Data Analysis, 25:465-490. 